\title{
Superconducting Magnets for
}

Magnetic Density Separation A NbTi based demonstrator

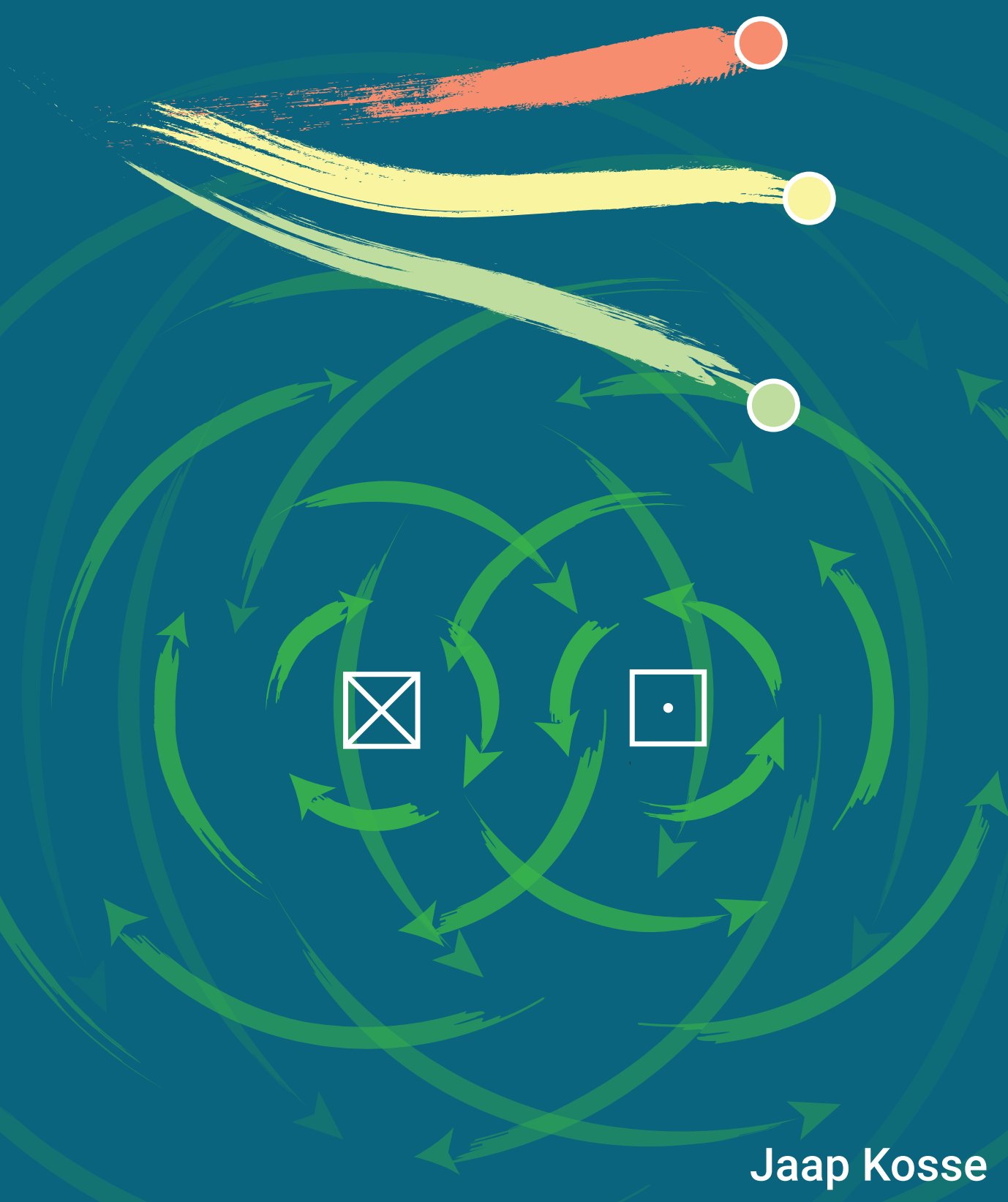




\section{SUPERCONDUCTING MAGNETS FOR MAGNETIC DENSITY SEPARATION}

A NbTi based demonstrator 
This dissertation has been approved by:

Supervisors: $\quad$ prof.dr.ir. Marcel ter Brake

prof.dr.ir. Herman ten Kate

Co-supervisor: $\quad$ dr. Marc Dhallé

The research described in this thesis was carried out at:

The University of Twente, Faculty of Science and Technology, chair of Industrial Application of Superconductivity and chair Energy, Materials \& Systems, Enschede, The Netherlands.

Printed by: Ipskamp Printing

ISBN: $\quad$ 978-90-365-5148-9

DOI: $\quad \underline{10.3990 / 1.9789036551489}$

(C) 2021 Jaap Kosse, The Netherlands. All rights reserved. No parts of this thesis may be reproduced, stored in a retrieval system or transmitted in any form or by any means without permission of the author. Alle rechten voorbehouden. Niets uit deze uitgave mag worden vermenigvuldigd, in enige vorm of op enige wijze, zonder voorafgaande schriftelijke toestemming van de auteur. 


\title{
SUPERCONDUCTING MAGNETS FOR MAGNETIC DENSITY SEPARATION
}

A NbTi based demonstrator

\section{DISSERTATION}

\author{
to obtain \\ the degree of doctor at the Universiteit Twente, \\ on the authority of the rector magnificus, \\ prof. dr. ir. A. Veldkamp, \\ on account of the decision of the Doctorate Board \\ to be publicly defended \\ on Thursday 25 March 2021 at 16.45 hours
}

by

\section{Jaap Jeroen Kosse}

born on the $29^{\text {th }}$ of June 1991

in Hardenberg, the Netherlands 


\section{Graduation Committee}

Chairman/secretary: prof.dr.ir. J.L. Herek University of Twente

Supervisors: $\quad$ prof.dr.ir. H.J.M. ter Brake University of Twente prof.dr.ir. H.H.J. ten Kate University of Twente

Co-supervisor: $\quad$ dr. M.M.J. Dhallé University of Twente

Committee Members: prof.dr. K.J. Boller University of Twente prof.dr.ir. J.W.M. Hilgenkamp University of Twente prof.dr.ir. P.C. Rem University of Delft prof.dr. L. Rossi University of Milan prof.dr. J.H. Snoeijer University of Twente 


\section{Contents}

Nomenclature $\quad$ v

1 Introduction 1

1.1 Introduction . . . . . . . . . . . . . . . . 1

1.2 Magnetic density separation . . . . . . . . . . . . 1

1.3 Improving MDS: Dutch Technology Foundation STW research

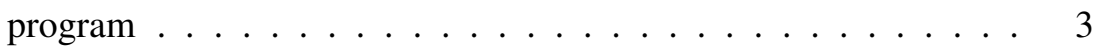

1.4 Scope of thesis: superconducting MDS . . . . . . . . . . . . . 3

1.5 Structure of thesis . . . . . . . . . . . . . . . . 5

2 Ideal magnetic field for MDS

$\begin{array}{ll}\text { and approximations } & 9\end{array}$

2.1 Introduction . . . . . . . . . . . . . . . 10

2.2 MDS operating principle . . . . . . . . . . . . . 10

2.3 Ideal magnetic field and the required 2D current distribution . . . 13

2.4 Racetrack coils: optimization of the 2D current distribution . . . . 16

2.4.1 Optimal discrete racetrack geometry . . . . . . . . . 16

2.4.2 Effect of winding pack thickness . . . . . . . . . . . . 19

2.4.3 Further approximation of ideal current distribution . . . . 26

2.5 Conclusion . . . . . . . . . . . . . . 30

3 Electromagnetic design $\quad 33$

3.1 Number- and type of racetrack coils . . . . . . . . . . . . 34

3.1 .1 Magnet-driven MDS . . . . . . . . . . . . . 35

3.1.2 Potential coil layouts for MDS . . . . . . . . . . 35

3.1.2.1 Practical layouts of racetrack coils. . . . . . . . 36

3.1.2.2 Variation in vertical magnetic field gradient . . . 40

3.1.2.3 Effect of tilting the plane of coils . . . . . . . 43

3.1 .3 3D analysis . . . . . . . . . . . . . . . 47

3.1.4 Winding pack geometry and composition ....... 50 
3.1 .5 Summary .................... 53

3.2 Estimate of potential performance of superconducting MDS . . . 54

3.2.1 Structure of the performance estimate analysis . . . . . 56

3.2.2 Minimizing the peak magnetic field in head sections (step 2) ......................... 59

3.2.3 Temperature margin under operating conditions (step 3) . 60

3.2.4 Obtainable current density and magnetic field at conductor (step 4) . . . . . . . . . . . . . . . 63

3.2.5 Performance of the NbTi demonstrator magnet . . . . . . 64

3.2.6 Magnetic field at fluid bed (step 5) . . . . . . . . . . 65

3.2.7 Lorentz force and coil thickness (step 6) . . . . . . . . . 65

3.2.8 Required cassette thickness (step 7) . . . . . . . . . . 70

3.2.9 Performance - demonstrator extrapolation (step 8) . . . 70

3.2 .10 Summary . . . . . . . . . . . . . . . . 75

3.3 Attraction force between coils and ferrofluid . . . . . . . . . 75

3.3.1 Analytical expression for the coils-to-fluid force . . . . 75

3.3.2 Unstable ferrofluid load-case . . . . . . . . . . . . . . 77

3.3.3 Numerical calculation of coil-fluid attraction for angled

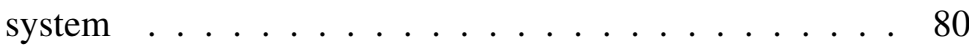

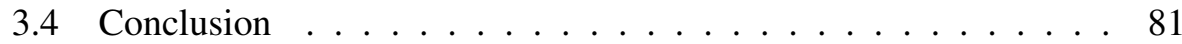

4 Mechanical design $\quad 85$

4.1 Introduction . . . . . . . . . . . . . . . . 86

4.2 Cryostat . . . . . . . . . . . . . . . . . . . 89

4.3 Winding pack composite properties . . . . . . . . . . . . 94

4.4 Mechanical behaviour of the cassette . . . . . . . . . . . . . 96

4.4.1 Simulation set-up . . . . . . . . . . . . . . . 100

4.4.2 Contact conditions . . . . . . . . . . . . . . . 102

4.4 .3 Material properties . . . . . . . . . . . . . . . . 102

4.4 .4 Loads . . . . . . . . . . . . . . . . . . . . . 103

4.4.5 Simulation results . . . . . . . . . . . . . 106

4.4.5.1 Aluminium alloy cassette . . . . . . . . . 106

4.4.5.2 Winding pack . . . . . . . . . . . 106

4.4.5.3 Clamping the coils - bolts \& threaded rods . . . 110

4.4.6 Effect of cassette thickness . . . . . . . . . . . . . . 112

4.4.7 Asymmetric cassette design attempts . . . . . . . . . . 117

4.5 Cold mass support structure . . . . . . . . . . . . . . . . . . 120

4.5.1 Buckling: critical load . . . . . . . . . . . . . . . 121

4.5.2 Optimized pillar dimensions against buckling . . . . . . . 123

4.5.3 Vertical \& horizontal pillar structures . . . . . . . . . 126

4.5.4 Resonance frequencies of cassette-pillar structure . . . . . 128 
4.5.5 Response of the cold mass to shocks . . . . . . . . . . 130

4.5.6 Feasibility of vibration isolation . . . . . . . . . . . 133

4.6 Conclusion . . . . . . . . . . . . . . . . 136

5 Thermal \& electrical design 139

5.1 Introduction . . . . . . . . . . . . . . . . . . . . 140

5.2 Thermal design . . . . . . . . . . . . . . . . . . 141

5.2 .1 Cryocooler . . . . . . . . . . . . . . . 141

5.2.2 Heat loads . . . . . . . . . . . . . . . . . . . 142

5.2.2.1 Support structure . . . . . . . . . . . . . . 143

5.2.2.2 Radiation . . . . . . . . . . . . . . . 143

5.2.2.3 Current leads . . . . . . . . . . . . . . . . 145

5.2.2.4 Instrumentation . . . . . . . . . . . . . . 148

5.2.2.5 Ramping losses . . . . . . . . . . . . . . . . 149

5.2.3 Cooling budget - first estimation . . . . . . . . . . . . . 149

5.2 .4 Lumped thermal model . . . . . . . . . . . . . . . . 151

5.2 .5 Cold mass temperature . . . . . . . . . . . . . . 158

5.2 .6 Cool-down time . . . . . . . . . . . . . . . . 162

5.3 Electrical circuit . . . . . . . . . . . . . . . . . 165

5.3.1 Self-inductance and stored energy . . . . . . . . . . 165

5.3.2 MDS magnet's electrical circuit . . . . . . . . . . . 166

5.3.3 Current lead temperature during fault . . . . . . . . . 168

5.3.4 Simulation of a quenching coil . . . . . . . . . . 168

5.3.4.1 Adiabatic winding pack . . . . . . . . . . . 170

5.3.4.2 Thermally connected winding pack . . . . . . 175

5.4 Conclusion . . . . . . . . . . . . . . . . . . . 182

6 Manufacturing of NbTi MDS racetrack coils 183

6.1 Introduction . . . . . . . . . . . . . . . . . . . . . . 184

6.2 Coil winding procedure . . . . . . . . . . . . . . . . 184

6.3 Diagnosis of electrical short . . . . . . . . . . . . . . 188

6.4 Effects of electrical short . . . . . . . . . . . . . . . . . 192

6.5 Room-temperature impedance . . . . . . . . . . . . . . 196

6.6 Conclusion . . . . . . . . . . . . . . . . . . . 199

7 Conclusion \& outlook 201

$\begin{array}{lll}\text { Appendix A Scaling laws } & \mathbf{2 0 7}\end{array}$

A.1 NbTi . . . . . . . . . . . . . . . . . . . . . 207

A.2 $\mathrm{Nb}_{3} \mathrm{Sn} \ldots \ldots \ldots \ldots \ldots \ldots \ldots$

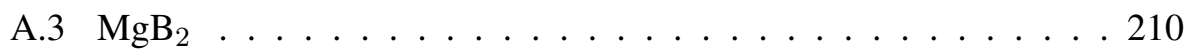




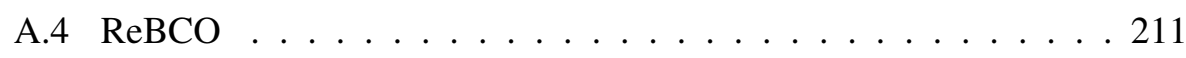

Appendix B Magnetic field profile 213

$\begin{array}{ll}\text { Bibliography } & 227\end{array}$

$\begin{array}{ll}\text { Summary } & 235\end{array}$

$\begin{array}{ll}\text { Samenvatting } & 239\end{array}$

$\begin{array}{ll}\text { Acknowledgements } & 243\end{array}$ 


\section{Nomenclature}

\section{Symbols}

\begin{tabular}{|c|c|c|}
\hline Symbol & Units & Description \\
\hline$a$ & $\mathrm{~m}$ & $\begin{array}{l}\text { scaling factor between peak magnetic field } \\
\text { and current density }\end{array}$ \\
\hline$a_{n}$ & - & Fourier coefficient \\
\hline$B$ & $\mathrm{~T}$ & magnetic flux density \\
\hline$B$ & $\mathrm{~T}$ & magnetic flux density magnitude \\
\hline$b_{n}$ & - & Fourier coefficient \\
\hline$C$ & $\mathrm{~F}$ & capacitance \\
\hline$D_{\mathrm{i}}$ & $\mathrm{m}$ & inner diameter \\
\hline$D_{\mathrm{o}}$ & $\mathrm{m}$ & outer diameter \\
\hline$d$ & $\mathrm{~m}$ & winding pack thickness or cryostat top plate thickness \\
\hline$d_{\text {cas }}$ & $\mathrm{m}$ & cassette plate thickness \\
\hline$d_{\text {coil }}$ & $\mathrm{m}$ & winding pack thickness \\
\hline$d z$ & $\mathrm{~m}$ & cryostat top plate deflection \\
\hline$E$ & GPa & Young's modulus \\
\hline$E_{\mathrm{c}}$ & $\mathrm{V} / \mathrm{m}$ & electric field criterion \\
\hline$g$ & $\mathrm{~m} / \mathrm{s}^{2}$ & gravitational constant \\
\hline $\boldsymbol{F}_{\text {buoyancy }}$ & $\mathrm{N}$ & buoyant force \\
\hline$F_{\mathrm{L}}$ & $\mathrm{N}$ & Lorentz force \\
\hline $\boldsymbol{F}_{\text {mag }}$ & $\mathrm{N}$ & magnetic force \\
\hline $\boldsymbol{F}_{z}$ & $\mathrm{~N}$ & gravitational force \\
\hline$F_{\text {cr }}$ & $\mathrm{N}$ & critical buckling load \\
\hline$f_{\text {mag }}$ & $\mathrm{N} / \mathrm{m}^{3}$ & magnetic force density \\
\hline
\end{tabular}




\begin{tabular}{|c|c|c|}
\hline$f$ & $\mathrm{~Hz}$ & resonance frequency \\
\hline$G$ & $\mathrm{GPa}$ & shear modulus \\
\hline $\boldsymbol{H}$ & $\mathrm{A} / \mathrm{m}$ & magnetic field \\
\hline$H$ & $\mathrm{~A} / \mathrm{m}$ & magnetic field magnitude \\
\hline$I$ & $\mathrm{~A}$ & current \\
\hline$I_{\mathrm{c}}$ & $\mathrm{A}$ & critical current \\
\hline$I_{\mathrm{m}}$ & $\mathrm{m}^{4}$ & moment of inertia \\
\hline$J$ & $\mathrm{~A} / \mathrm{m}^{2}$ & volume current density \\
\hline$J$ & $\mathrm{~A} / \mathrm{m}^{2}$ & volume current density magnitude \\
\hline$J_{\mathrm{c}}$ & $\mathrm{A} / \mathrm{m}^{2}$ & critical current density \\
\hline$J_{\mathrm{e}}$ & $\mathrm{A} / \mathrm{m}^{2}$ & engineering current density \\
\hline $\boldsymbol{K}$ & $\mathrm{A} / \mathrm{m}$ & sheet current \\
\hline$K$ & $\mathrm{~A} / \mathrm{m}$ & sheet current magnitude \\
\hline$K_{n}$ & $\mathrm{~A} / \mathrm{m}$ & sheet current Fourier coefficient \\
\hline$k$ & $\mathrm{~W} / \mathrm{m} / \mathrm{K}$ & thermal conductivity \\
\hline$k$ & $\mathrm{~N} / \mathrm{m}$ & stiffness \\
\hline$k$ & - & coupling coefficient between inductors \\
\hline$k_{n}$ & - & separation constant \\
\hline$L$ & $\mathrm{H}$ & self-inductance of magnet \\
\hline$L$ & $\mathrm{~m}$ & length \\
\hline$L_{\text {extra }}$ & $\mathrm{m}$ & extra length of racetrack leg sections \\
\hline$M_{\mathrm{s}}$ & $\mathrm{A} / \mathrm{m}$ & saturation magnetization \\
\hline$m$ & $\mathrm{~A} \cdot \mathrm{m}^{2}$ & magnetic dipole moment \\
\hline$N$ & - & safety factor against buckling \\
\hline$n$ & - & Fourier number \\
\hline$n$-value & - & steepness of superconducting-to-normal transition \\
\hline$P_{\text {hyst }}$ & $\mathrm{W}$ & hysteresis loss rate \\
\hline $\boldsymbol{P}_{\mathrm{mag}}$ & $\mathrm{Pa}$ & magnetic pressure \\
\hline$P_{\text {mag }}$ & $\mathrm{Pa}$ & magnetic pressure magnitude \\
\hline$p$ & - & packing factor \\
\hline$\dot{Q}$ & $\mathrm{~W}$ & heat load \\
\hline$R$ & $\Omega$ & electrical resistance \\
\hline$R_{\mathrm{c}}$ & $\mathrm{K} / \mathrm{W}$ & thermal contact resistance \\
\hline$T$ & $\mathrm{~K}$ & temperature \\
\hline
\end{tabular}




\begin{tabular}{|c|c|c|}
\hline$T_{\mathrm{cs}}$ & $\mathrm{K}$ & current sharing temperature \\
\hline$T_{\mathrm{op}}$ & $\mathrm{K}$ & operating temperature \\
\hline$t$ & $\mathrm{~s}$ & time \\
\hline$V$ & $\mathrm{~m}^{3}$ & feed particle volume or volume of top plate of cryostat \\
\hline$w$ & $\mathrm{~m}$ & $\begin{array}{l}\text { width of current-carrying section of } \\
\text { rectangular current distribution }\end{array}$ \\
\hline$w_{1}$ & $\mathrm{~m}$ & width of racetrack coil leg \\
\hline$w_{2}$ & $\mathrm{~m}$ & width of inner section of racetrack coil \\
\hline$X$ & - & measure of ferrofluid stability \\
\hline$x$ & $\mathrm{~m}$ & horizontal coordinate, flow direction \\
\hline$x^{\prime}$ & $\mathrm{m}$ & tilted horizontal coordinate, flow direction \\
\hline$y$ & $\mathrm{~m}$ & horizontal coordinate \\
\hline$z$ & $\mathrm{~m}$ & vertical coordinate \\
\hline$z^{\prime}$ & $\mathrm{m}$ & tilted vertical coordinate \\
\hline$z_{\mathrm{eq}}$ & $\mathrm{m}$ & particle equilibrium height \\
\hline$\alpha$ & $\mathrm{rad}$ & tilt angle of coils \\
\hline$\Delta z$ & $\mathrm{~m}$ & separation distance \\
\hline$\varepsilon$ & - & mechanical strain \\
\hline$\zeta$ & - & damping constant \\
\hline$\theta_{n}$ & - & phase Fourier coefficient \\
\hline$\lambda$ & $\mathrm{m}$ & characteristic length \\
\hline$\mu_{0}$ & $\mathrm{H} / \mathrm{m}$ & permeability of free space \\
\hline$\nu$ & - & Poisson's ratio \\
\hline$\rho$ & $\Omega \cdot \mathrm{m}$ & electrical resistivity \\
\hline$\rho_{\mathrm{fl}}$ & $\mathrm{kg} / \mathrm{m}^{3}$ & ferrofluid mass density \\
\hline$\rho_{\mathrm{p}}$ & $\mathrm{kg} / \mathrm{m}^{3}$ & feed particle mass density \\
\hline$\rho_{\max }$ & $\mathrm{kg} / \mathrm{m}^{3}$ & maximum feed particle mass density \\
\hline$\rho_{\min }$ & $\mathrm{kg} / \mathrm{m}^{3}$ & minimum feed particle mass density \\
\hline$\sigma$ & $\mathrm{Pa}$ & mechanical stress \\
\hline$\chi$ & - & magnetic susceptibility \\
\hline$\psi$ & $\mathrm{A}$ & scalar magnetic potential \\
\hline$\psi_{n}$ & - & Fourier coefficient of phase \\
\hline
\end{tabular}




\section{Abbreviations}

$\begin{array}{ll}\text { Al } & \text { aluminium } \\ \text { AL5083 } & \text { aluminium alloy } \\ \text { BSCCO } & \text { bismuth strontium calcium copper oxide, superconductor } \\ \text { Bi-2212 } & \text { type of BSCCO } \\ \text { Bi-2223 } & \text { type of BSCCO } \\ \text { Cernox } & \text { type of temperature sensor } \\ \text { Cu } & \text { copper } \\ \text { FEM } & \text { finite element method } \\ \text { Formvar } & \text { polyvinyl formal } \\ \text { G11 } & \text { type of laminated composite of epoxy and glass fabric } \\ \text { HTS } & \text { high temperature superconductor } \\ \text { HX } & \text { heat exchanger } \\ \text { LTS } & \text { low temperature superconductor } \\ \text { MDS } & \text { magnetic density separation } \\ \text { MgB } 2 & \text { magnesium diboride, superconductor } \\ \text { Nb } 3 \text { Sn } & \text { niobium-tin, superconductor } \\ \text { NbTi } & \text { niobium-titanium, superconductor } \\ \text { OFHC } & \text { oxygen-free high thermal conductivity copper } \\ \text { PM } & \text { permanent magnet } \\ \text { Pt100 } & \text { type of temperature sensor } \\ \text { ReBCO } & \text { rare-earth barium copper oxide, superconductor } \\ \text { RRR } & \text { residual resistance ratio } \\ \text { RSD } & \text { relative standard deviation } \\ \text { SS304 } & \text { AISI304 stainless steel } \\ \text { SS316 } & \text { AISI316 stainless steel } \\ & \end{array}$




\section{1 | Introduction}

\subsection{Introduction}

$\mathrm{T}^{\mathrm{N}}$ this thesis the research performed to develop the first superconducting magnet 1 for use in Magnetic Density Separation (MDS), a novel separation technology, is described. The MDS process is introduced in Section 1.2, and the program performed for improving this technology in Section 1.3. In Section 1.4 the focus is on the research questions addressed in this thesis, and in Section 1.5 it is on the structure of this work.

\subsection{Magnetic density separation}

$\mathrm{M}$ AGNETIC density separation (MDS) is a novel recycling technology that allows to separate a mixture of non-magnetic materials based on their mass density [1-6] and that ideally requires a magnetic field with a magnitude that only changes in the vertical direction.

The separation or filtration of magnetic materials with the aid of high-gradient magnetic fields is a well-established and widely-used technology [7, 8] that was developed in the seventies and eighties of last century [9, 10]. It essentially exploits the attraction of ferro-, ferri- or paramagnetic particles in the direction of the magnetic field gradient and is used for the purification of for example coal [1113], ores [14-16] and waste water [17, 18] or in the manipulation of various organic and biological materials [19-21]. Another widely used magnetic separation technique is eddy-current repulsion, mostly used to extract non-ferrous metals out of waste streams [22, 23].

The separation of non-magnetic particles is enabled by the use of a ferrofluid in combination with a high-gradient magnetic field [24], where the magnetic force on the fluid competes with gravity (or centrifugal forces) to separate materials based on their specific density [8]. Before MDS, this method was limited to a binary sink-float approach. A major advantage of the MDS technology compared to other types of magnetic separation is its ability to separate multiple components 
in a one-step process.

All these methods require a strong magnetic field gradient. What distinguishes MDS is that this gradient does not just need to be strong, but ideally also onedirectional. As discussed in Chapter 2, variation of the magnetic field in a horizontal plane tends to re-mix the feed stream and hence needs to be minimized. This requirement is unique to MDS and leads to a specific design of the magnet.

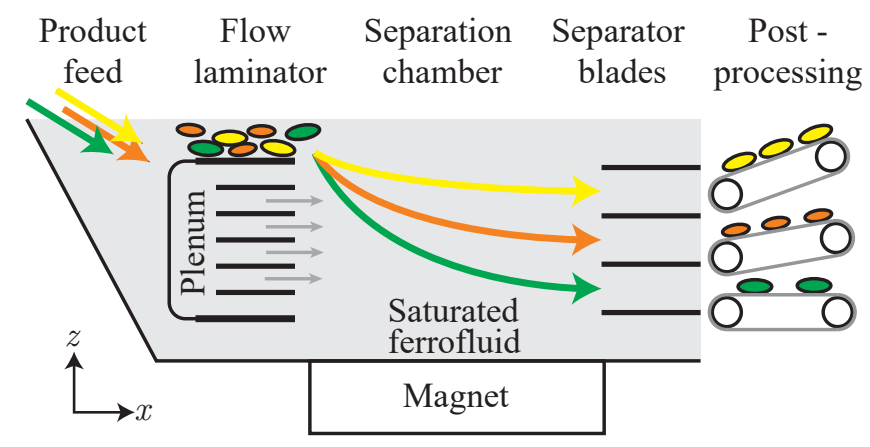

Figure 1.1: Schematic representation of an MDS system. Feed material is shredded, wetted, and immersed into a ferrofluid flow. A magnet creates a vertical magnetic field gradient in the ferrofluid [1]. Particles move towards a certain height $z$ that corresponds to their mass density. They can then be collected in different groups by separator blades.

In the MDS process, illustrated in Figure 1.1, shredded feed particles are immersed in a superparamagnetic fluid (ferrofluid) that flows over a magnet. The fluid is a colloid of superparamagnetic nanoparticles, usually water-based [25], that is magnetically saturated using a magnet that generates a vertical magnetic field gradient [2]. The competition between gravity - acting on the feed particlesand magnetic attraction - acting on the ferrofluid-leads to a net force on the feed material that pushes it up to an equilibrium height $z_{\mathrm{eq}}$, which depends on its mass density. Different-density particles thus float at different heights in the fluid bed.

A fluid flow drags the mixed feed stream from its insertion point towards separator blades that collect with a certain efficiency the different-density constituent materials. The ferrofluid is then recovered and the separated feed products go through further sensor sorting for final purification [6]. A transport belt moves over the magnet at the same speed as the fluid to reduce turbulence and to carry away any magnetic materials that may be present in the feed stream. 


\subsection{Improving MDS: Dutch Technology Foundation STW research program}

7 HE research of which the results are presented in this thesis was performed within the framework of the research program Innovative Magnetic Density Separation for the Optimal Use of Resources and Energy, financed (partly) by the Dutch Technology Foundation STW [26]. The main goal of the program is to improve the MDS technology in order to enhance recycling and save energy.

The program consists of seven projects, spearheaded by four universities in the Netherlands:

1. Superconducting MDS laboratory demonstrator, University of Twente;

2. Liquid-cryogen free superconducting magnet prototype, University of Twente;

3. Ultra-low turbulence ducts for magnetic density separation, University of Eindhoven;

4. Particle Fluid Particle Interaction, University of Eindhoven;

5. Chemical design of magnetic nanoparticles with high colloidal stability under MDS conditions, University of Utrecht;

6. Understanding the physics of ultra-fast mechanical fine particle sorting of MDS products, University of Delft;

7. Demonstration of scalable process systems able to deliver a very large number of pure, high value products with just a few process steps, University of Delft.

The work in this thesis falls within the scope of the first project. Besides cooperation with the other university partners, and in particular with the University of Delft, corporate partners within the STW research project include Urban Mining Corporation and Sumitomo Electric.

\subsection{Scope of thesis: superconducting MDS}

C TATE-OF-THE-ART MDS systems use permanent magnets, usually in the form $\checkmark$ of Halbach arrays $[1,27,28]$, which imposes limits in terms of pole size and magnetic field strength. Superconductivity can significantly enhance the separation resolution while allowing for feeds of wider density ranges or for more dilute ferrofluids, resulting in cost reduction. 
This thesis is focused on the design and construction of a conduction-cooled NbTi-based MDS demonstrator magnet for the separation of electronic material, e.g. shredded motherboards, that will constitute the world's first superconducting MDS system. A schematic of the complete system is shown in Figure 1.2.

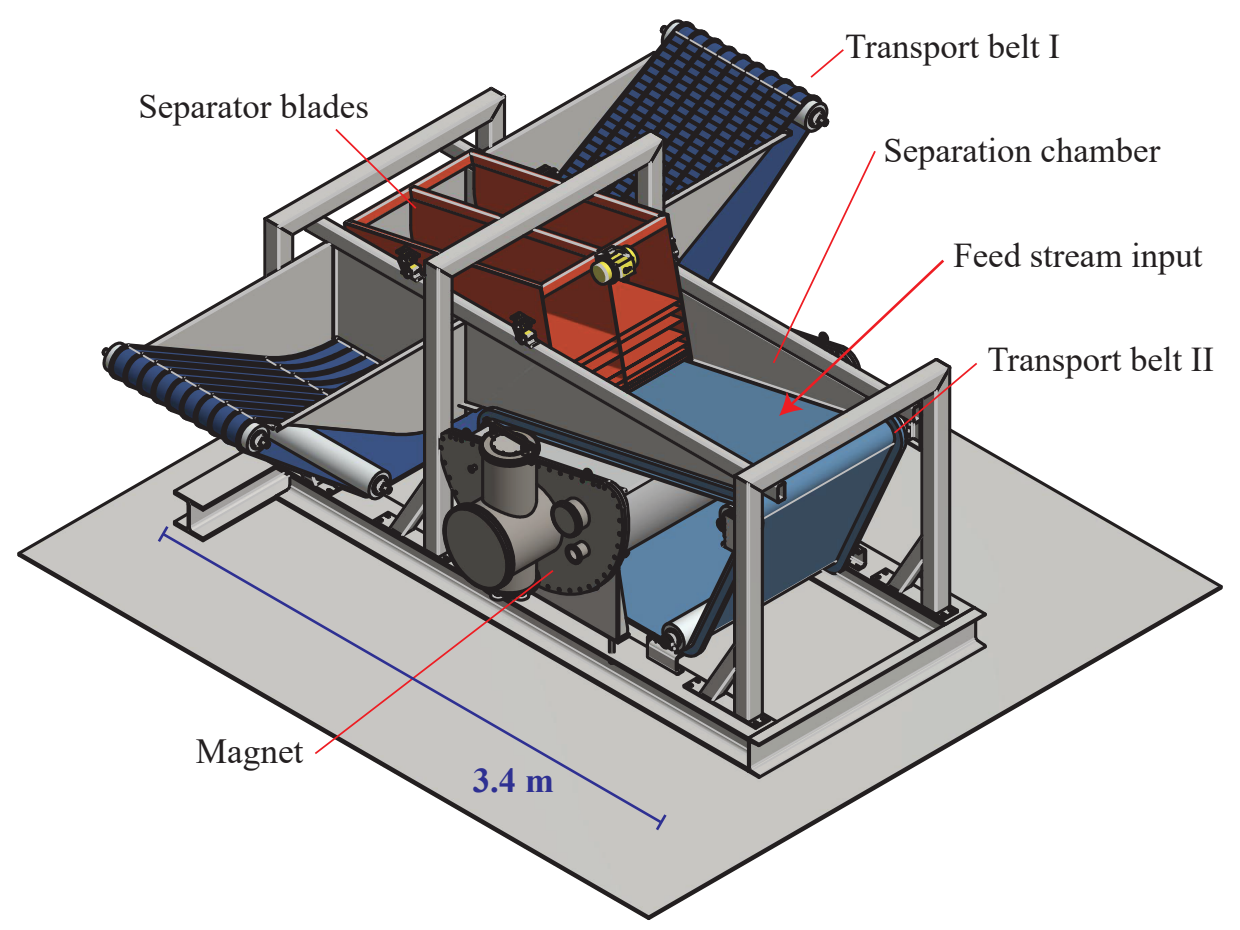

Figure 1.2: Schematic 3D representation of an MDS system [29].

The magnet essentially consists of three $\mathrm{NbTi} / \mathrm{Cu}$ flat racetrack coils, each with a width of $0.3 \mathrm{~m}$ and a length of $1.4 \mathrm{~m}$. The peak magnetic field in the coils is $5.2 \mathrm{~T}$ and the operating current $300 \mathrm{~A}$. The magnet will operate at $4.5 \mathrm{~K}$, cooled by a closed-cycle cryocooler. The coils generate an average magnetic field of $2.0 \mathrm{~T}$ at the bottom of the fluid bed, with a vertical magnetic field gradient of magnitude $20 \mathrm{~T} / \mathrm{m}$. The fluid bed is $0.9 \mathrm{~m}$ long in the flow direction, $1.0 \mathrm{~m}$ wide and $0.3 \mathrm{~m}$ deep.

Compared to the current permanent-magnet based MDS systems, which have a $0.6 \mathrm{~T}$ magnetic field at the fluid bed bottom and a pole size of $0.12 \mathrm{~m} \mathrm{[3]}$, this design offers an increase in the separation resolution by a factor 2.5 , defined as the vertical distance between the equilibrium heights of two particles with different mass densities. 
While superconducting systems have been developed for a variety of other separation systems [30-34], their required magnetic field profiles are different from that of MDS.

The presented work is closely linked to the construction of the demonstrator magnet. Unfortunately, final system integration and commissioning are not covered in this thesis as these occurred after completion of the present $\mathrm{PhD}$ work.

This thesis is written in such a way that it can be used as a foundation in the design of future superconducting magnets for MDS.

The main research questions addressed are:

- What is the optimum shape of the magnetic field required for MDS and how can this magnetic field be approximated by using a practical arrangement of coils in an electromagnet?

- How can the distance between the ferrofluid bath and the coils be minimized? This minimization of the distance between the large planar surfaces formed by the top surface of the coils and the bottom of the ferrofluid bed is needed in order to maximize the performance. This minimization challenge is unique to MDS;

- What magnitude of the vertical magnetic field gradient can be expected from coils based on available technical superconductors?

- What magnetic attraction force will arise between the coils and the ferrofluid, and how to cope with that?

- How can the coils effectively be cooled and kept at the desired operating temperature of $4.5 \mathrm{~K}$ ? This operating temperature was thought to be a realistic achievable temperature at the start of the project.

- Which mechanical support structure of the cold mass in its vacuum enclosure can provide adequate support while not resulting in a prohibitively high heat in-leak to the cold mass or in a too large distance between coils and ferrofluid?

\subsection{Structure of thesis}

THIS thesis concerns five main chapters 2 to 6 , as illustrated in Figure 1.3. 1 These chapters chronologically describe the research that has been performed. In Chapter 2 the ideal magnetic field for MDS and a current distribution that can generate this magnetic field are derived. This current distribution is approximated by an infinite array of racetrack coils. 
Taking this array as a starting point, Chapter 3 investigates the optimal number and type of racetrack coils, also taking into account edge effects. It includes the potential performance of various technical superconductors for MDS, as well as the attraction force between the ferrofluid and the coils. The performance analysis partly relies on results of the mechanical design (Chapter 4) and the thermal design (Chapter 5).

Chapter 4 focuses on the mechanical aspects of the magnet. The main design challenge is to minimize the distance between the flat coil surface and the flat bottom of the ferrofluid bath.

In Chapter 5 the thermal and electrical aspects of the system are presented. It is shown that the system can operate conduction-cooled using a single cryocooler. in this way, a single-walled vacuum vessel can be used and thereby the coil-tofluid distance can be small $(50 \mathrm{~mm})$. This chapter also presents the chosen quench protection strategy.

As a first and crucial step in the realization of the demonstrator, the manufacturing of the three $\mathrm{NbTi} / \mathrm{Cu}$-based coils at the University of Twente is discussed in Chapter 6. 


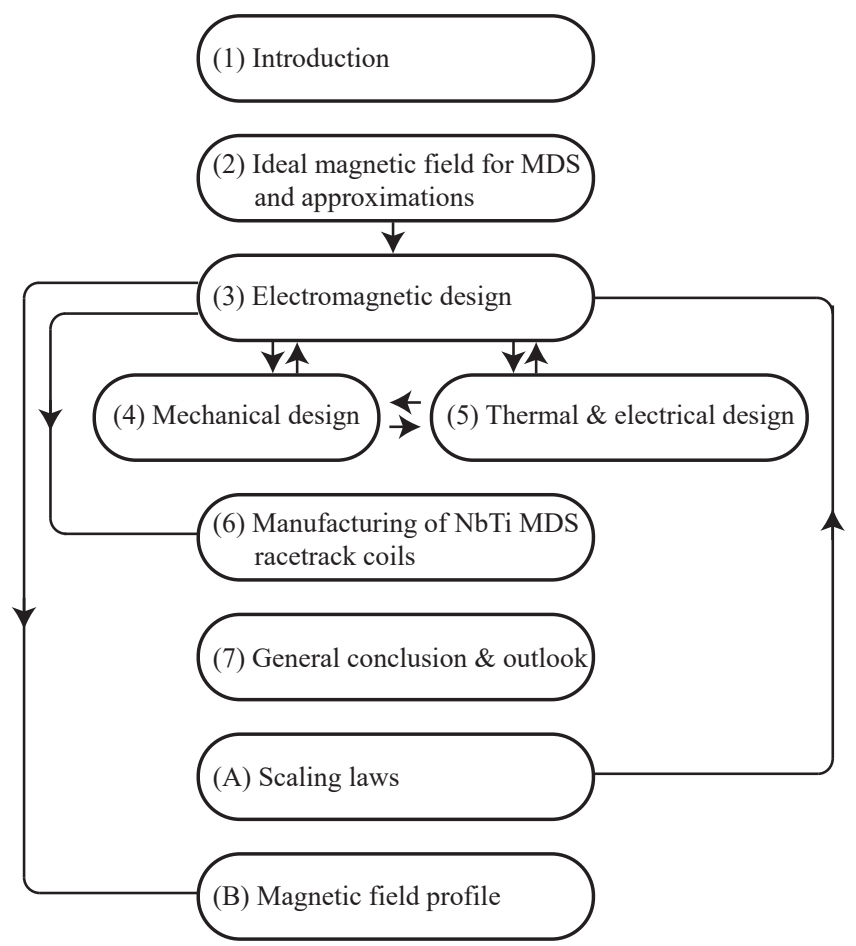

Figure 1.3: Schematic overview of the chapters that make up this work. Arrows indicate the dependence of chapters on each other. 


\section{Ideal magnetic field for MDS and approximations}

In this chapter electromagnet layouts are presented that are able to generate a vertical magnetic field gradient that does not vary significantly in the horizontal plane but changes with the vertical coordinate. This magnetic field shape is required for MDS.

As a starting point in the design process an ideal current distribution that fulfils the magnetic field requirements is derived. It is shown that in order to eliminate wiggles in feed particle trajectories, a harmonic sheet current is required. The generated magnetic field decays exponentially with the distance from the sheet current. This ideal layout is then approximated by practical coil configurations. The approximations are done using Fourier analysis and result in an optimal cross-sectional profile when using a layout based on flat racetrack coils. Included is the effect of the winding pack thickness on the magnetic field at the fluid bed and on the peak magnetic field.

The presented work is applicable not just for use in this recycling technology, but for any application that requires a strong vertical magnetic field gradient that changes only in the vertical direction. 


\subsection{Introduction}

THIS chapter outlines how the electromagnetic design of the magnet relates

I to the desired magnetic field profile for this application. In Section 2.2 the MDS operating principle is briefly presented, sketching how the forces on a feed particle are affected by the magnetic field profile and showing why the field magnitude ideally should change only with the vertical distance to the magnet, but not in a horizontal plane. The discussion also further clarifies how superconducting electromagnets may enhance this new technology.

In Section 2.3 it is presented analytically how the generation of such an ideal magnetic field profile in principle requires an infinitely extended harmonic sheet current.

In Section 2.4 it is shown how such an ideal current distribution can best be approximated with a real magnet system. First Fourier analysis is used to optimize 2D current patterns that represent a system of flat racetrack-type magnets of varying complexity, then the thickness of the winding pack in a real 3D implementation is taken into account, maximizing the magnitude of the useful magnetic field above the magnet and minimizing the peak field within the winding pack.

\subsection{MDS operating principle}

CONSIDER a particle with volume $V$ and mass density $\rho_{\mathrm{p}}$, submerged in a ferrofluid with density $\rho_{\mathrm{fl}}$ (Figure 2.1). In the absence of a magnetic field $H$, a particle denser than the ferrofluid will sink to the bottom of the fluid bed because the sum $\boldsymbol{F}_{z}+\boldsymbol{F}_{\text {buoyancy }}$ of the gravitational- and buoyant forces is directed downwards. When a magnet underneath the fluid is switched on, the ferrofluid is attracted towards it. This causes an additional upward force $\boldsymbol{F}_{\text {mag }}$ on the nonmagnetic particle.

This upward force is the opposite of the magnetic force that would act on the same volume of ferrofluid placed at the same location. It is given by [35]

$$
\boldsymbol{F}_{\text {mag }}=-\nabla(\boldsymbol{m} \cdot \boldsymbol{B})
$$

with $\boldsymbol{m}$ the magnetic dipole moment of the displaced ferrofluid volume and $\boldsymbol{B}$ the local magnetic flux density. The ferrofluid is assumed to be magnetically saturated to a value $M_{\mathrm{s}}$ in the direction of the local magnetic field, so

$$
\boldsymbol{m} \cdot \boldsymbol{B}=V M_{\mathrm{s}} B(z)
$$

With $\nabla M_{\mathrm{s}}=0$ and $\nabla B(z)=\mu_{0} \nabla H(z)$ the magnetic force on the particle 


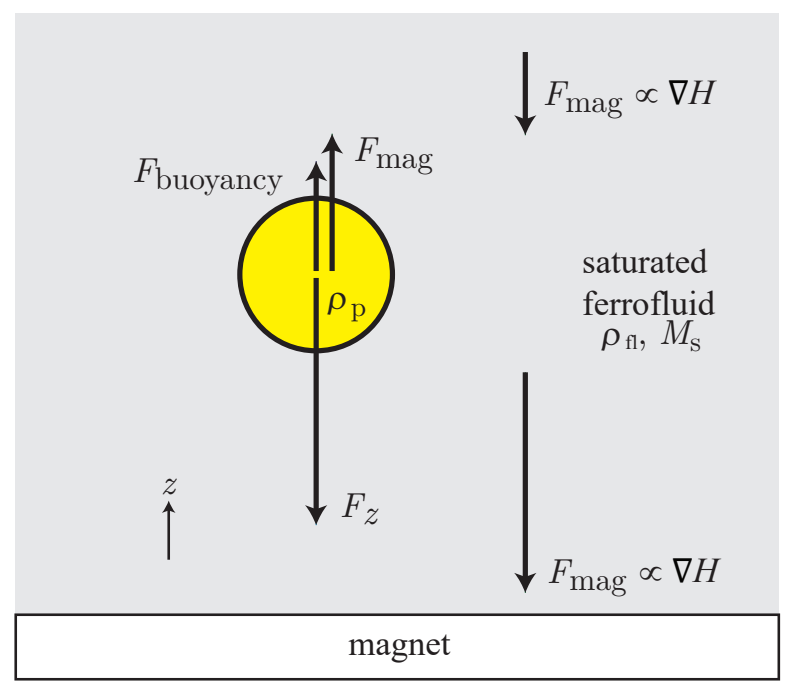

Figure 2.1: Forces acting on a non-magnetic particle that is submerged in a magnetized ferrofluid in a vertical magnetic field gradient. The buoyant force and gravity are independent of height $z$, whereas the magnetic force decays with increasing height.

becomes

$$
\boldsymbol{F}_{\mathrm{mag}}=-\mu_{0} M_{\mathrm{s}} V \nabla H(z)
$$

Note that this force is exerted on the non-magnetic particle by the surrounding fluid, not directly by the magnetic field. It can thus be regarded as a heightdependent buoyancy force ${ }^{1}$.

At a certain equilibrium height $z_{\mathrm{eq}}$ the net force on the particle in the vertical direction is zero [2], yielding

$$
\begin{aligned}
0 & =F_{z}+F_{\text {buoyancy }}+F_{\mathrm{mag}}\left(z=z_{\mathrm{eq}}\right) \\
& =\left(\rho_{\mathrm{fl}}-\rho_{\mathrm{p}}\right) V g-\mu_{0} M_{\mathrm{s}} V \nabla_{z} H\left(z=z_{\mathrm{eq}}\right),
\end{aligned}
$$

with $g$ the gravitational acceleration. This expression can be simplified further by assuming a magnetic field with a magnitude that decays exponentially with

\footnotetext{
${ }^{1}$ The separation of materials with a density lower than that of the ferrofluid can be achieved by placing an (additional) magnet on top of the fluid bed [2].
} 
height, with a characteristic decay length $\lambda$

$$
H(z)=H_{0} \exp \left(-\frac{2 \pi}{\lambda} z\right) .
$$

The reasoning behind this choice is made clear in Section 2.3. By inserting the (vertical) gradient of this magnetic field in the force balance (Eq. 2.4b), one obtains a closed expression for the equilibrium height

$$
z_{\mathrm{eq}}=\frac{\lambda}{2 \pi} \ln \left[\frac{2 \pi \mu_{0} M_{\mathrm{s}} H_{0}}{\left(\rho_{\mathrm{p}}-\rho_{\mathrm{fl}}\right) g \lambda}\right] .
$$

For such an exponentially decaying field, $z_{\text {eq }}$ does not depend on the horizontal coordinates $(x, y)$, so that particles do not 'wiggle' up and down as they are moving through the fluid bed. Also, since the magnetic force changes with the vertical coordinate, different-density particles will indeed float at different heights, which is the basis of the MDS process. In Section 2.3 it is shown that an exponential field as in Eq. 2.5 is actually the only possible solution that meets these requirements perfectly.

The saturation magnetization $M_{\mathrm{s}}$ and the field strength $H_{0}$ appear in Eq. 2.6 as a product. This allows to find an optimal balance between capital- and operational expenditure of an MDS installation. The first is dominated by the cost of the magnet system, the second by the price of the ferrofluid [36]. Note that even though the fluid is recirculated, a fraction is lost during the cleaning of the sorted feed particles. Since superconducting magnets can generate stronger fields than PM systems, more dilute ferrofluids can be used. Also, a stronger field allows to lift—and thus to separate—heavier materials.

From Eq. 2.6 the vertical distance between the equilibrium heights of two different materials with densities $\rho_{1}$ and $\rho_{2}$, the separation distance, $\Delta z$, can straightforwardly be derived as

$$
\Delta z=z_{\mathrm{eq}}\left(\rho_{2}\right)-z_{\mathrm{eq}}\left(\rho_{1}\right)=\frac{\lambda}{2 \pi} \ln \left(\frac{\rho_{1}-\rho_{\mathrm{fl}}}{\rho_{2}-\rho_{\mathrm{fl}}}\right) .
$$

This distance gives an indication of the obtainable separation resolution. As discussed in Section 2.4, the decay length $\lambda$ of the magnetic field strength scales with the size of the poles of the magnet, which is relatively limited for PM. Electromagnets in principle have no inherent size limitation, since coils can be wound in arbitrary dimensions. A larger $\lambda$-value allows for an increased separation distance $\Delta z$ for feed streams with a given density range $\left[\rho_{1}, \rho_{2}\right]$, enhancing the separation resolution compared to magnets with a more limited $\lambda$.

The next section considers the relation between the current distribution, i.e. the layout of an electromagnet, and the magnetic field profile in more detail. 


\subsection{Ideal magnetic field and the required 2D current dis- tribution}

$\mathrm{T}$ this section it is shown how the optimal magnetic field for MDS is indeed 1 described by Eq. 2.5. The vertical gradient of the magnetic field magnitude should not depend on the horizontal coordinates $(x, y)$, so that the equilibrium height of the feed particles is constant in the horizontal plane. This minimizes particle 'wiggling' and thus improves separation accuracy. We refer to such a magnetic field profile as the 'ideal' field ${ }^{2}$. Mathematically, this requirement is expressed as

$$
\frac{\partial^{2} H}{\partial x \partial z}=0, \quad \frac{\partial^{2} H}{\partial y \partial z}=0 .
$$

First we consider the general case of a magnetic field above a unidirectional periodic sheet current with an arbitrary shape. From this, the sheet current distributions that satisfy Eq. 2.8 are identified, showing that only a pure harmonic sheet current generates such a field and that hence only an exponentially decaying field meets these requirements.

Consider a periodic sheet current $\boldsymbol{K}=K(x) \hat{\mathbf{y}}$ that flows at $z=0$ in the $y$-direction in an infinite $x y$-plane and is described the Fourier series

$$
K(x)=\sum_{n=0}^{\infty} K_{n} \cos \left(n \frac{2 \pi}{\lambda} x+\phi_{n}\right) .
$$

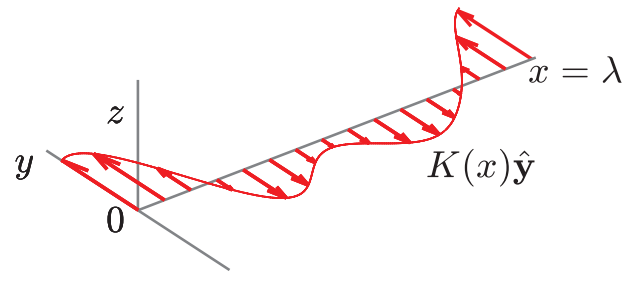

Figure 2.2: Arbitrary sheet current $\boldsymbol{K}(x)$ with a magnitude that varies periodically with the horizontal coordinate $x$ with period $\lambda$, such that $\forall x: \boldsymbol{K}(x+\lambda)=$ $\boldsymbol{K}(x)$.

\footnotetext{
${ }^{2}$ Horizontal field gradients are also of interest, since they can slow down, and even reverse, particle motion over the fluid bed. The main source of horizontal gradients is the truncation of the infinite $x y$-plane to a finite-size magnet system, which is explored further in Section 3.1.1.
} 
Above the current sheet, Ampére's law dictates that $\nabla \times \boldsymbol{H}=0$, since in this region the current density is zero. This means a scalar magnetic potential $\psi$ can be invoked to solve the field

$$
\boldsymbol{H}=\nabla \psi
$$

by solving the Laplace equation

$$
\nabla^{2} \psi=0
$$

A standard approach to solve Eq. 2.11 is by separation of variables, $\psi(x, z)=$ $X(x) Z(z)$ [35]. The general solution to Eq. 2.10 and Eq. 2.11 can be written as

$$
\begin{aligned}
& H_{x}=\sum_{n=0}^{\infty} \cos \left(k_{n} x+\theta_{n}\right)\left(a_{n} e^{+k_{n} z}+b_{n} e^{-k_{n} z}\right), \\
& H_{z}=\sum_{n=0}^{\infty} \sin \left(k_{n} x+\theta_{n}\right)\left(a_{n} e^{+k_{n} z}-b_{n} e^{-k_{n} z}\right) .
\end{aligned}
$$

These equations are valid in general for any current-free region. The phases $\phi_{n}$ and the amplitudes $a_{n}$ and $b_{n}$ need to be derived from the boundary conditions, while the $k_{n}$ are separation constants.

Invoking once more Ampere's law, this time at the current sheet, the boundary condition at $z=0$ can be expressed as

$$
K(x)=\lim _{z \downarrow 0} H_{x}(x, z)-\lim _{z \uparrow 0} H_{x}(x, z)=2 \lim _{z \downarrow 0} H_{x}(x, z),
$$

where the first limit represents the magnetic field just above the sheet and the second one just below. In combination with Eq. 2.9 and Eq. 12, Eq. 13 yields $k_{n}=2 \pi n / \lambda ; \theta_{n}=\phi_{n}$ and $a_{n}+b_{n}=K_{n} / 2$. Moreover, for a practical planar current distribution as in Fig. 2.2, currents need to return to the source so that the net current in the $y$-direction is zero and the trivial solution with $n=0$ (corresponding to a uniform field generated by a uniform current sheet) may be excluded. In that case, the requirement that the field magnitude $H$ remains finite for $z \uparrow \infty$ implies that all coefficients $a_{n}$ must be zero, and the field generated by the periodic sheet current (Eq. 2.9) becomes

$$
\begin{aligned}
& H_{x}=+\sum_{n=1}^{\infty} \frac{K_{n}}{2} \cos \left(n \frac{2 \pi}{\lambda} x+\phi_{n}\right) e^{-k_{n} z}, \\
& H_{z}=-\sum_{n=1}^{\infty} \frac{K_{n}}{2} \sin \left(n \frac{2 \pi}{\lambda} x+\phi_{n}\right) e^{-k_{n} z} .
\end{aligned}
$$


This is the general expression for the fields generated by periodic sheet currents of the type of Eq. 2.9. We can now verify which of these currents lead to a magnetic field that meets the requirement of Eq. 2.8, i.e. which currents lead to a one-directional field gradient. The magnitude of the field can be written as

$$
\begin{aligned}
H= & \frac{1}{2}\left\{\sum_{n=1}^{\infty} \sum_{m=1}^{\infty} K_{n} K_{m} \cos \left[(n-m) \frac{2 \pi}{\lambda} x+\phi_{n}-\phi_{m}\right]\right. \\
& \left.\times \exp \left[-(n+m) \frac{2 \pi}{\lambda} z\right]\right\}^{0.5} .
\end{aligned}
$$

with partial derivate in the $z$-direction

$$
\begin{aligned}
\frac{\partial H}{\partial z}= & \frac{1}{2 H} \frac{\partial H^{2}}{\partial z}=-\frac{\pi}{4 H} \sum_{n=1}^{\infty} \sum_{m=1}^{\infty}(n+m) K_{n} K_{m} \\
& \times \cos \left[(n-m) \frac{2 \pi}{\lambda} x+\phi_{n}-\phi_{m}\right] \exp \left[-(n+m) \frac{2 \pi}{\lambda} z\right] .
\end{aligned}
$$

We require this function to be independent of $x$ (see Criterion 2.8). If $n$ and $m$ are equal, the cosine terms are independent of $x$ and there are no restrictions on $K_{n}$. However, if $n$ and $m$ are unequal this will not be the case. One must therefore make sure that these cross-terms do not contribute.

For every contribution in the dual sum where $n$ and $m$ are unequal, the cosineterm has to be extinguished by the coefficients that determine its amplitude. This can not be done by the factor $(n+m)$, which is always positive. Thus we need to place demands on the product of the coefficients $K_{n}$ and $K_{m}$

$$
\sum_{n=1}^{\infty} \sum_{\substack{m=1 \\ m \neq n}}^{\infty} K_{n} K_{m}=0 .
$$

This means that only a single term $K_{n}$ can be non-zero. In other words, only a pure harmonic sheet current will produce a field with a purely one-directional magnitude gradient. 
In that case, one may as well set $K_{1} \neq 0$, choose the phase angle $\phi_{1}$ to be zero, and write

$$
\begin{aligned}
\boldsymbol{K} & =K_{1} \cos \left(\frac{2 \pi}{\lambda} x\right) \hat{\mathbf{y}} \\
H_{x} & =+\frac{K_{1}}{2} \cos \left(\frac{2 \pi}{\lambda} x\right) \exp \left(-\frac{2 \pi}{\lambda} z\right) \\
H_{z} & =-\frac{K_{1}}{2} \sin \left(\frac{2 \pi}{\lambda} x\right) \exp \left(-\frac{2 \pi}{\lambda} z\right), \\
H & =\frac{K_{1}}{2} \exp \left(-\frac{2 \pi}{\lambda} z\right)
\end{aligned}
$$

\subsection{Racetrack coils: optimization of the 2D current distri- bution}

R EALIZING a cosine-shaped sheet current distribution as described by Eq. 2.18 tion more realistic current distributions are considered that approximate such an ideal current distribution. The corresponding deviations from the ideal magnetic field (Eq. 2.19-2.21) can be expressed in terms of Fourier coefficients.

First, in Section 2.4.1, the effects of moving from a continuous to a discrete 2D current distribution are considered. This allows to model an infinite array of thin racetrack-coil and thus to optimize the shape of the coils. Then, in Section 2.4.2, the effect of a finite winding pack thickness (in the $z$-direction, see Figures 2.2 and 2.3) is included, switching from a $2 \mathrm{D}$ sheet current to a $3 \mathrm{D}$ volume current and exploring the consequences both for the useful field above the coils and for the peak field within the winding pack. Also this analysis will still consider an infinite array of coils. End-effects caused by truncating the periodic current distribution to a finite number of coils (in the $x$-direction) with a finite length (in the $y$-direction) are discussed in Section 3.1.1.

\subsubsection{Optimal discrete racetrack geometry}

A magnet system that is practical to produce comprises a series of flat racetrack coils, as shown in Figure 2.3. These are electro-magnets that consist of two straight coil sections, each with a width $w$, which are connected by two semicircular 'heads'. Assuming a homogeneous current distribution in the winding pack allows to model the magnetic field produced by such a coil accurately, so one does not need to consider the individual wires within the winding pack [37] ${ }^{3}$.

\footnotetext{
${ }^{3}$ On condition that a correct packing factor is taken into account.
} 
In the analysis below, we focus on the $x z$-cross section corresponding to the $y$ symmetry plane ${ }^{4}$.

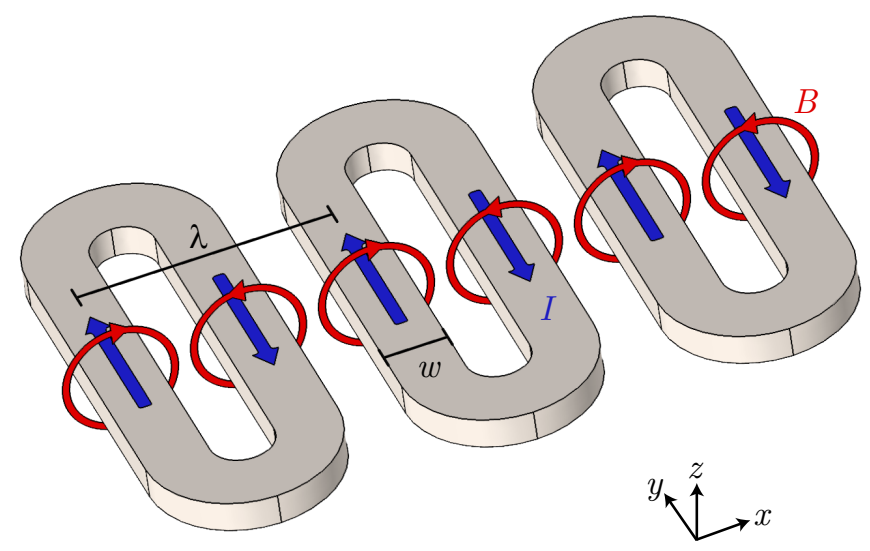

Figure 2.3: Sketch of a magnet consisting of 3 flat racetrack coils. The current direction is shown by blue straight arrows, the magnetic field direction by red circular arrows. Coils are translated by a distance $\lambda$ relative to each other in the $x$-direction.

Consider an infinite number of such racetrack coils, translated a distance $\lambda$ from each other in the $x$-direction. In this first subsection, the relative thickness of the coils $d / \lambda$ is still assumed to be small enough to approximate the volume current density $\boldsymbol{J}$ by a surface current density $\boldsymbol{K}(x)=\boldsymbol{J}(x) d$. The corresponding sheet current then consists of alternating regions where $\boldsymbol{K}(x)$ is either $+K_{0} \hat{\mathbf{y}}$, zero or $-K_{0} \hat{\mathbf{y}}$ with periodicity $\lambda$, as in Figure 2.4. The question that can then be addressed is which racetrack leg width $w$ yields the best approximation of the ideal field.

To determine an optimal value for $w / \lambda$, the discrete Fourier components of the sheet current are of interest. To answer this question, we develop this current distribution as a Fourier series, like in Eq. 2.9. Choosing $x=0$ in the center of one of the racetrack legs, we can exploit the symmetry $K(x)=K(-x)$ to set all the phases $\phi_{n}$ to zero

$$
K(x)=\sum_{n=1}^{\infty} K_{n} \cos \left(n \frac{2 \pi}{\lambda} x\right),
$$

\footnotetext{
${ }^{4}$ In an MDS system, the direction of the fluid flow could be either in the $x$ - or $y$-direction.
} 


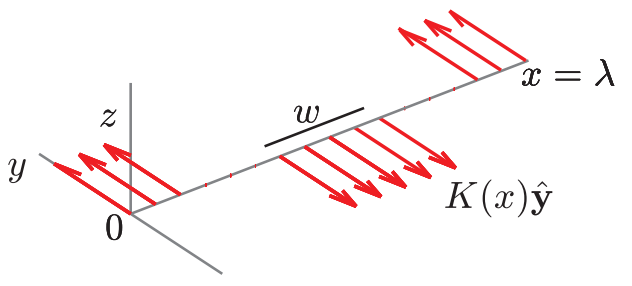

Figure 2.4: Sheet current $\boldsymbol{K}(x)$ that has a magnitude varying stepwise with the horizontal coordinate $x$ with period $\lambda$. This rectangular current distribution represents one period of the coil layout of the racetracks in Figure 2.3.

where, as discussed above, we omitted the $n=0$ term to take into account that the net current in the $y$-direction is zero.

For a current distribution as in Figure 2.4, the Fourier coefficients $K_{n}$ can straightforwardly be derived to be

$$
K_{n}=\frac{2 K_{0}}{n \pi} \sin \left(n \frac{w}{\lambda} \pi\right)[1-\cos (n \pi)] .
$$

Here $K_{0}$ is a scaling term. It is set to 1 in the remainder of the section for simplicity. An example of such a Fourier expansion is shown in Figure 2.5, for the specific case $w / \lambda=1 / 3$. Note that the components with $n$ even are zero.

The field generated by this current distribution can then be derived from Eqs. 2.14a and 2.14b. As shown by Eq. 2.16, the presence of the higher harmonics $n=3,5, \ldots$ leads to an undesired $x$-component of $\nabla H$. It is therefore important to minimize the influence of $K_{3}$ and higher components. However, compared to our desired $n=1$ term these higher harmonics decay fast with the height $z$ above the magnet, due to the factor $n$ in the exponent. In other words, at larger distances lower-order Fourier components contribute more to the magnetic field than the higher-order ones. To approximate the ideal field as closely as possible, it is therefore especially important to minimize the lower-order harmonics.

In Figure 2.6 the first terms of the Fourier expansion are shown as a function of the current sheet width $w / \lambda$. By investigating Eq. 2.23, it can be seen that the $n^{\text {th }}$ component has zero magnitude whenever $w / \lambda=1 / n$. Thus by setting the width of the current sheets equal to exactly one-third of the coil system periodicity, $w / \lambda=1 / 3$, the amplitude of the normally dominant third harmonic can be made zero (and, more general, all $K_{3+6 m}$ with $m$ integer).

Note that this does not necessary mean that a racetrack coil needs a leg width of $\lambda / 3$. One can also use racetracks with leg width $\lambda / 6$ placed adjacent to each other, with the current flowing in the same direction for touching legs. 

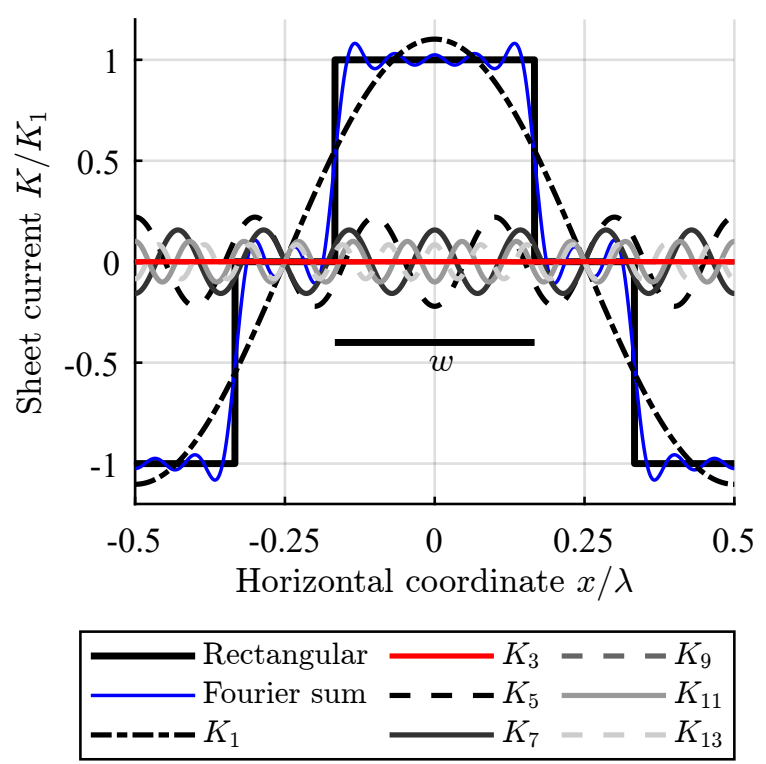

Figure 2.5: Rectangular (solid black line) sheet current distribution with periodicity $\lambda$ and width $w=\lambda / 3$. The current distribution can be expanded into a Fourier series, which terms (up to $n=13$ ) are plotted. The sum of the terms is the solid thin blue line. The $K_{1}$ term (dashed-dotted black line) represents the ideal current distribution.

Plotting the magnitude of the remaining Fourier components for $w / \lambda=1 / 3$ results in Figure 2.7. With $K_{3}$ removed, $K_{5}$ is the largest remaining unwanted component. A practical way of eliminating this component is by adding five subdivisions of period $\lambda / 5$ and amplitude $\frac{2}{5 \pi} \sin \left(\frac{5}{3} \pi\right)$ to the original current sheet profile. This type of current distribution will be called a notched distribution. However, eliminating the $K_{5}$ (and possibly higher order Fourier coefficients) comes at the price of a more complex winding pack. More details on this such further refinement of the current distribution can be found in Section 2.4.3.

\subsubsection{Effect of winding pack thickness}

A real coil has of course a finite winding pack thickness $d$ and carries a volumetric current density instead of a sheet current. This has consequences for the magnetic field in the fluid bed, where generally the highest possible magnitude is desired, but also for the field experienced by the conductor from which the coil is wound. Note that in superconducting electromagnets the peak magnetic field in the windings limits their current-carrying properties and should therefore be kept as low as 

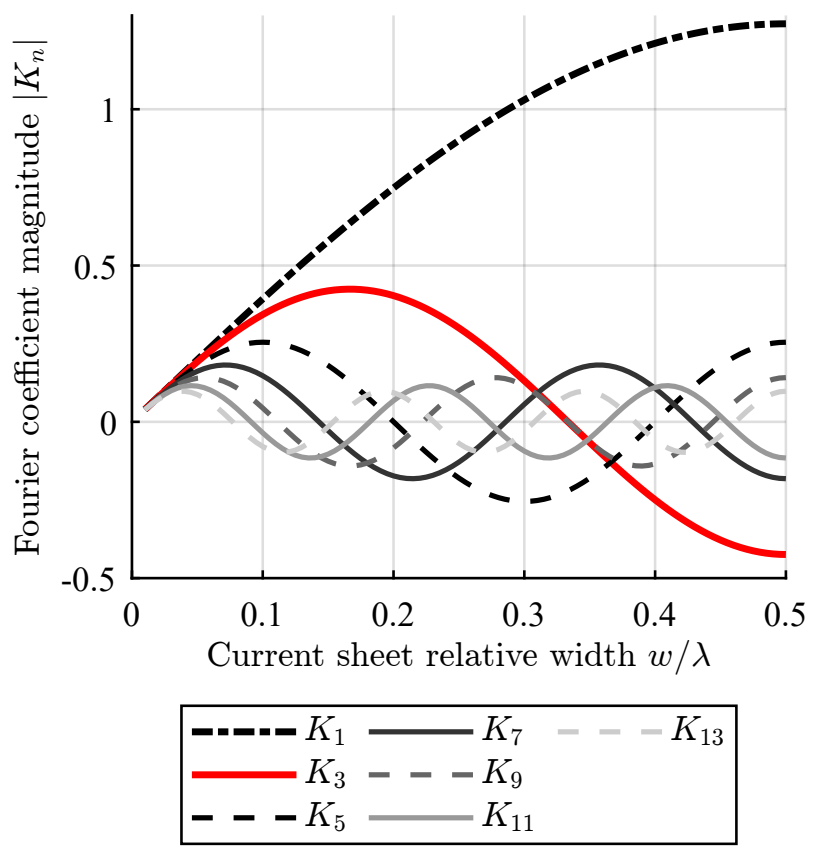

Figure 2.6: Variation of the magnitude of the magnetic field Fourier components as a function of current sheet width. The $n^{\text {th }}$ component is zero at $w / \lambda=1 / n$.

possible [38, 39].

For the magnetic field in the fluid bed, the effect of the winding pack thickness on the field magnitude is of interest, as well as-for the rectangular current distributions - the change in ripple, which as discussed above is represented by the higher-order Fourier coefficients. Inside the winding pack, we will show how the effect on the peak magnetic field differs for the ideal- and for the rectangular current distributions.

We start once more by considering the ideal pure harmonic current configuration and work out the magnetic field magnitude at a height $z$ above the conductor when we gradually increase the winding pack thickness $d$ while keeping the current density $J_{0}$ in the winding pack constant.

This can be done by integrating the field contributions generated by a series of parallel sheet currents of infinitesimal thickness $\mathrm{d} z^{\prime}$ located in the range $z^{\prime}=$ $[-d, 0]$, each carrying a current amplitude $K_{1}=J_{0} \mathrm{~d} z^{\prime}$. Note that with this choice of coordinates, $z=0$ corresponds to the top of the winding pack. Referring to 


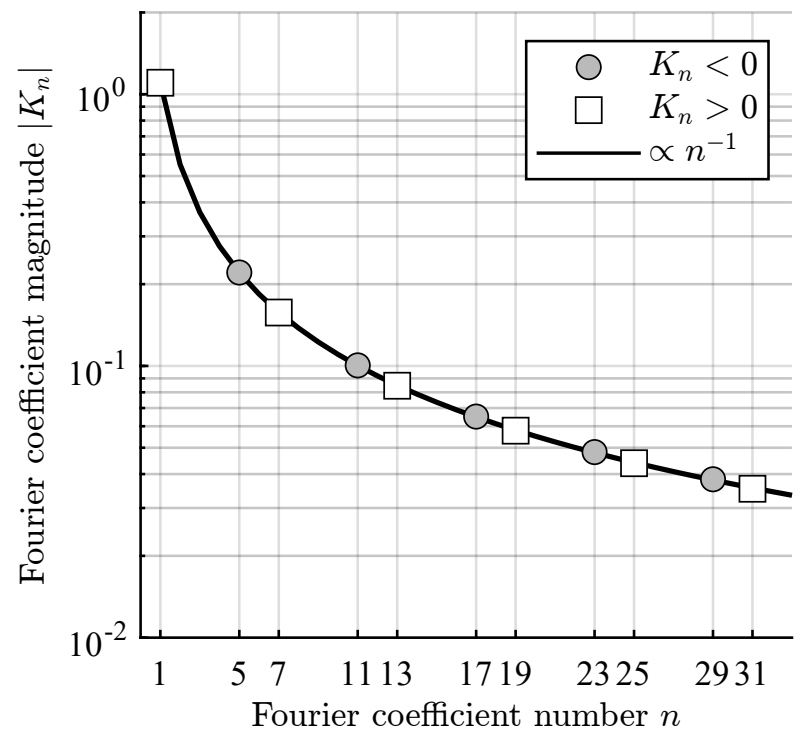

Figure 2.7: Variation of the magnitude of non-zero Fourier components for the rectangular current distribution in Figure 2.4, for a width $w / \lambda=1 / 3$. Components are plotted up to $n=31$. Square markers indicate positive values, round markers negative values.

Eqs. 2.19 and 2.20, we can write

$$
\begin{aligned}
H_{x} & =\frac{J_{0}}{2} \cos \left(\frac{2 \pi}{\lambda} x\right) \int_{z^{\prime}=-d}^{z^{\prime}=0} \exp \left(-\frac{2 \pi}{\lambda}\left(z-z^{\prime}\right)\right) \mathrm{d} z^{\prime} \\
& =\frac{J_{0} \lambda}{4 \pi} \cos \left(\frac{2 \pi}{\lambda} x\right) \exp \left(-\frac{2 \pi}{\lambda} z\right)\left[1-\exp \left(-\frac{2 \pi}{\lambda} d\right)\right],
\end{aligned}
$$

and analogously

$$
\begin{aligned}
H_{z} & =-\frac{J_{0} \lambda}{4 \pi} \sin \left(\frac{2 \pi}{\lambda} x\right) \exp \left(-\frac{2 \pi}{\lambda} z\right)\left[1-\exp \left(-\frac{2 \pi}{\lambda} d\right)\right], \\
H & =-\frac{J_{0} \lambda}{4 \pi} \exp \left(-\frac{2 \pi}{\lambda} z\right)\left[1-\exp \left(-\frac{2 \pi}{\lambda} d\right)\right] .
\end{aligned}
$$

Note that in the limit $d \rightarrow 0$ the expression reduces to Eq. 2.21 .

The value of the field magnitude just above the winding pack (at $z=0$ ) is plotted against winding pack thickness in Figure 2.8. For coils much thinner than $\lambda / 2 \pi$ the magnitude increases linearly with coil thickness, as may be expected, but for coils much thicker than $\lambda / 2 \pi$ additional windings added at the bottom 
are too far away to contribute significantly and the field magnitude saturates at $H=J_{0} \lambda / 4 \pi$.

The magnetic field components inside the current-carrying space $(-d \leq z \leq$ $0)$ are

$$
\begin{aligned}
& H_{x}=+\frac{J_{0} \lambda}{4 \pi} \cos \left(\frac{2 \pi}{\lambda} x\right)\left[\exp \left(\frac{2 \pi}{\lambda} z\right)-\exp \left(-\frac{2 \pi}{\lambda}(z+d)\right)\right] \\
& H_{z}=-\frac{J_{0} \lambda}{4 \pi} \sin \left(\frac{2 \pi}{\lambda} x\right)\left[2-\exp \left(\frac{2 \pi}{\lambda} z\right)-\exp \left(-\frac{2 \pi}{\lambda}(z+d)\right)\right] .
\end{aligned}
$$

These expressions are obtained by summing two integrals, the first spanning $[-d, z]$, the second $[z, 0]$. It can be proved that the maximum magnetic field is located at $z=-d / 2$ by solving $\partial H^{2} / \partial x=0$ and $\partial H^{2} / \partial z=0$. The maximum magnetic field inside the current-carrying space is given by

$$
H_{\text {peak }}=\frac{J_{0} \lambda}{2 \pi}\left[1-\exp \left(-\frac{\pi}{\lambda} d\right)\right]
$$

Also, Eq. 2.29 is plotted in Figure 2.8. The rate of increase of the peak field with winding pack thickness decreases as the winding pack gets thicker, but saturates at a larger thickness compared to the field on the surface.

The ratio of the effective magnetic field magnitude at the surface of the coils to the peak magnetic field in the conductor scales as

$$
\frac{H_{z=0}}{H_{\text {peak }}} \propto \frac{1-\exp \left(-\frac{2 \pi}{\lambda} d\right)}{1-\exp \left(-\frac{\pi}{\lambda} d\right)}=1+\exp \left(-\frac{\pi}{\lambda} d\right) .
$$

As the winding pack is made thicker, the relative increase of the peak magnetic field is larger than the relative increase of the magnetic field at the surface and this holds for all thicknesses $d$. Intuitively one may expect that this result also holds for rectangular current distributions. However, analysis in detail of these distributions, for both the 'simple'- and notched versions, shows that this is not the case.

As a start, the mean magnitude of the magnetic field above the rectangularand notched configurations can be accurately approximated by Eq. $2.24 \mathrm{~b}$ when including a small scaling term $\sqrt{K_{1}} \approx 1.05$ (open circles in Figure 2.8).

What is significantly different, however, is the relation between the peak magnetic field in the rectangular volume current distribution and the winding pack thickness. This peak field can be shown to be, once more using integration of the 


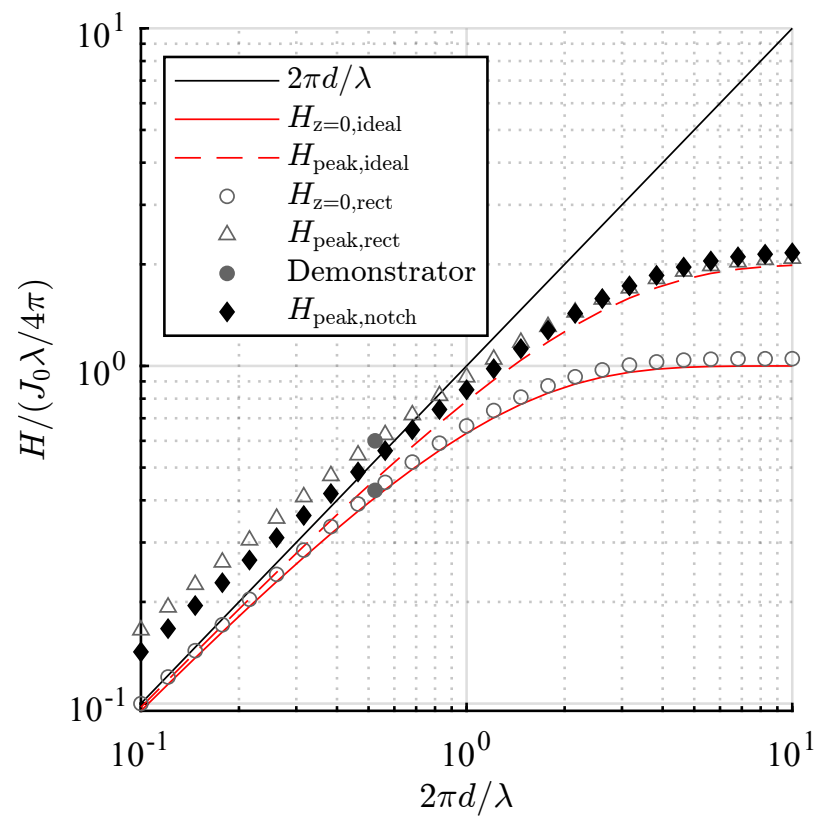

Figure 2.8: Scaled magnetic field magnitude as a function of coil thickness. The solid red line indicates the field at the surface of the coils for an ideal harmonic current distribution, the dashed red line the peak magnetic field in the conductor. The round markers indicate the field at the surface of the coils for a rectangular current distribution, the triangular markers the peak magnetic field in the conductor.

field components,

$$
H_{z=-d / 2, \text { rect }}=\frac{J_{0} \lambda}{2 \pi} \sum_{n=1}^{\infty} K_{n} \sin \left(n \frac{2 \pi}{\lambda} x\right)\left[1-\exp \left(-n \frac{\pi}{\lambda} d\right)\right] .
$$

This function varies with $x$, and of interest is the maximum

$$
H_{\text {peak,rect }}=\max _{-\lambda / 2 \leqslant x \leqslant \lambda / 2}\left[H_{z=-d / 2, \text { rect }}(x)\right] .
$$

This maximum as a function of coil thickness is shown in Figure 2.8, for both a rectangular- and notched configuration (both with $w / \lambda=1 / 3$ ). For thin winding packs a strong increase relative to the ideal current distribution is visible.

The higher-order harmonics decay quickly with distance so they do not influence the magnetic field significantly at the fluid bed, whereas they do affect the 
peak magnetic field in the winding pack. The thinner the coils are, the larger is the influence of the higher harmonics on the peak field. For a thicker winding pack the harmonics are generated at a larger distance from the peak field location, and due to their rapid decay their effect on the peak field is smaller.

By comparing the peak field of the notched configuration against the simple rectangular one, it can be observed that the effect of notching is biggest for thin winding packs. For thin coils, the $K_{5}$ component and its multiples $\left(K_{10}, K_{15}, \ldots\right)$ increase the peak field in the simple rectangular conductors, whereas for the notched configuration these harmonics are zero. For larger winding pack thicknesses these components have decayed relative to the $K_{1}$ component at the peak field location, and as such the notching has less effect on the peak field magnitude.

Some counter-intuitive behaviour due do the sharp increase of the peak magnetic field for thin coils can be seen when comparing the effective mean field magnitude at the surface of the coils with the peak field in the conductor. The ratio of these functions is plotted in Figure 2.9, for the rectangular- (blue) and notched (grey) configurations. They show a maximum at a non-zero winding pack thickness. Thus the intuitive result found for an ideal distribution does not hold: adding extra windings, i.e. making thicker coils, can provide a relative boost to the obtained gradient in the fluid larger than the accompanying increase in peak field in the conductor.

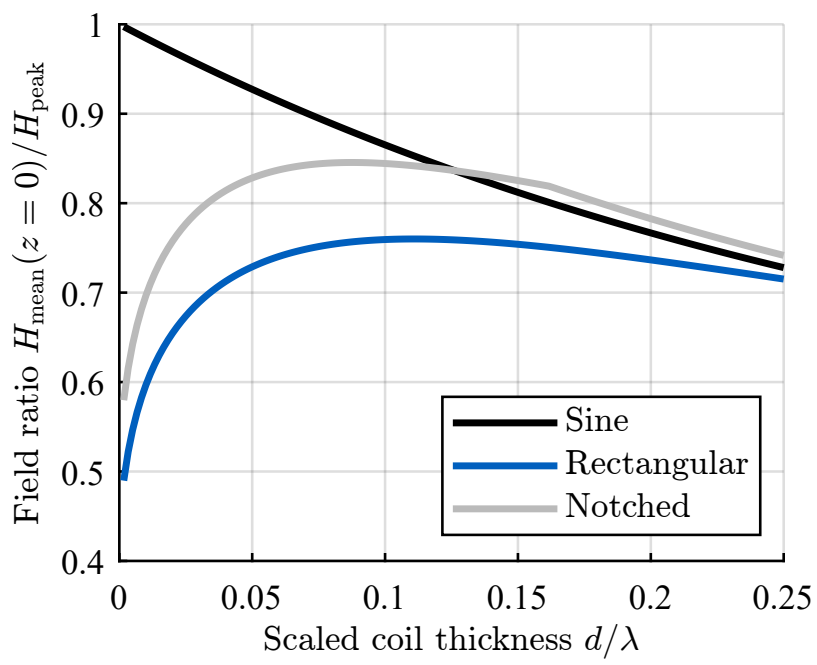

Figure 2.9: Ratio between the average magnetic field at the surface of the coils $(z=0)$ and the peak field within the coils, as a function of coil thickness.

Next the change in ripple of the magnetic field at the fluid bed as a result of the 
finite winding pack thickness is considered. As the magnetic field is ripple-free when generated by the 'ideal' sheet current distribution (Eq. 2.18), it will also be ripple-free when generated by a sum of parallel ideal sheet currents (i.e. an ideal volume current distribution).

For racetrack coils, moving from a sheet current to a volume current shows a change in the amplitude of the Fourier coefficients at the top of the winding pack. Each sheet current Fourier component is considered to be effectively reduced in magnitude at the top of the winding pack by a factor $\exp \left(-n \frac{2 \pi}{\lambda} z^{\prime}\right)$, where $z^{\prime}$ is the location of that current sheet

$$
\begin{aligned}
K_{n, \text { volume }}(z=0, d) & \\
= & \frac{K_{n}}{d} \int_{z^{\prime}=-d}^{z^{\prime}=0} \exp \left[-n \frac{2 \pi}{\lambda}\left(-z^{\prime}\right)\right] \mathrm{d} z^{\prime} \\
= & \frac{K_{n}}{d} \frac{\lambda}{2 \pi n}\left[1-\exp \left(-n \frac{2 \pi}{\lambda} d\right)\right] .
\end{aligned}
$$

In Figure 2.10 the effective magnitudes of the Fourier components are shown as a function of winding pack thickness, relative to the $K_{1}$ component at this particular thickness. At $d=\lambda / 12$, the value coinciding with our demonstrator magnet design, the contribution of the $K_{5}$ component is more than halved compared to its value for a single current sheet.

Moving from a sheet current to a volume current thus has a positive effect on the ratio of the effective fluid bed magnetic field to peak magnetic field, as well as on reducing the magnetic field ripple at the fluid bed.

To visualize the influence of a thicker winding pack, Figure 2.11 presents the magnetic field above a periodic array of racetrack coils with characteristic width $\lambda / 3$, for three different winding pack thicknesses.

The Fourier components of Eq. 2.33 are entered in Eq. 2.15 to obtain the expression for the magnetic field magnitude generated by a coil set with a nonzero winding pack thickness, valid for $z>0$ :

$$
\begin{aligned}
H= & \frac{1}{2}\left\{\sum_{n=1}^{\infty} \sum_{m=1}^{\infty}\left(\frac{\lambda}{2 \pi d}\right)^{2} \frac{K_{n} K_{m}}{n m}\left[1-\exp \left(-n \frac{2 \pi}{\lambda} d\right)\right]\right. \\
& \left.\times\left[1-\exp \left(-m \frac{2 \pi}{\lambda} d\right)\right] \cos \left[(m-n) \frac{2 \pi}{\lambda} x\right] \exp \left[-(m+n) \frac{2 \pi}{\lambda} z\right]\right\}^{0.5} .
\end{aligned}
$$

The Fourier components are given by Eq. 2.23. 


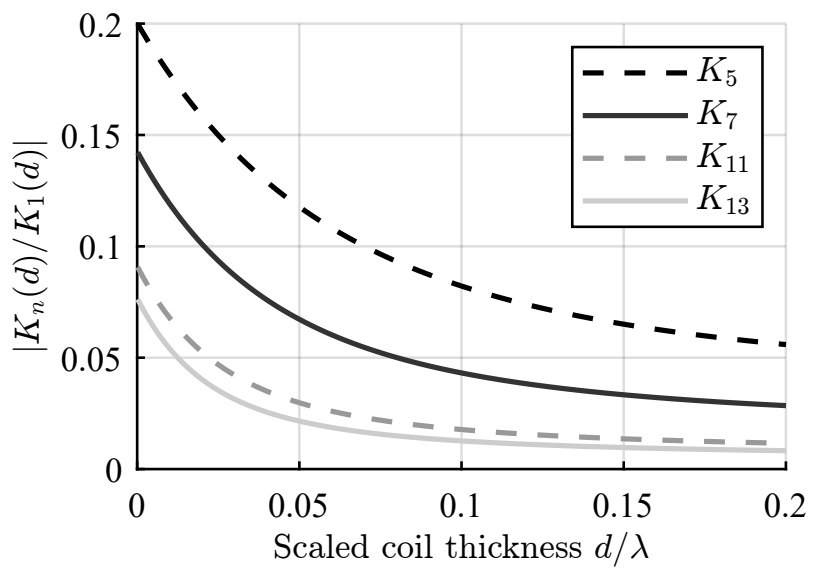

Figure 2.10: Magnitude of the non-zero Fourier components (up to $n=13$ ), plotted as a function of winding pack thickness, relative to the $K_{1}$ component at that thickness.

\subsubsection{Further approximation of ideal current distribution}

Here a current distribution is considered that, compared to the rectangular racetrack coils, still more closely resembles the ideal current distribution. By adding five subdivisions of period $\lambda / 5$ to the original rectangular current sheet profile, the current profile shown in Figure 2.12 is obtained. In practice this profile can be realized e.g. by shaping the winding pack cross-section, leaving out turns for certain $x$-values and adding turns elsewhere, so-called 'notching'.

The effect of this notching is that it removes the $K_{5}$ component completely. Looking again at the magnitudes of the Fourier components (Figure 2.7), it can be seen that the largest remaining coefficient after $K_{1}$ is now $K_{7}$ and it has a magnitude of $K_{1} / 7$ at $z=0$.

One could in principle divide the winding pack into even smaller segments, thereby eliminating the higher-order Fourier components. However, since the higher harmonics decay more quickly with the height $z$, the gain from eliminating these harmonics becomes smaller and is likely not worth the additional effort and added complexity. It will depend on the desired $\lambda$ whether the improvements made are worth this effort, as for smaller periodicities the near-coil region may be effectively enclosed in the cryostat.

Next focus is on the (dis)advantages of the notched current distribution. At a glance the notched configuration performs better than the pure rectangular one. For the same number of ampere-turns and the same average magnetic field magnitude at the fluid bed, operation with a lower peak magnetic field in the conductor 

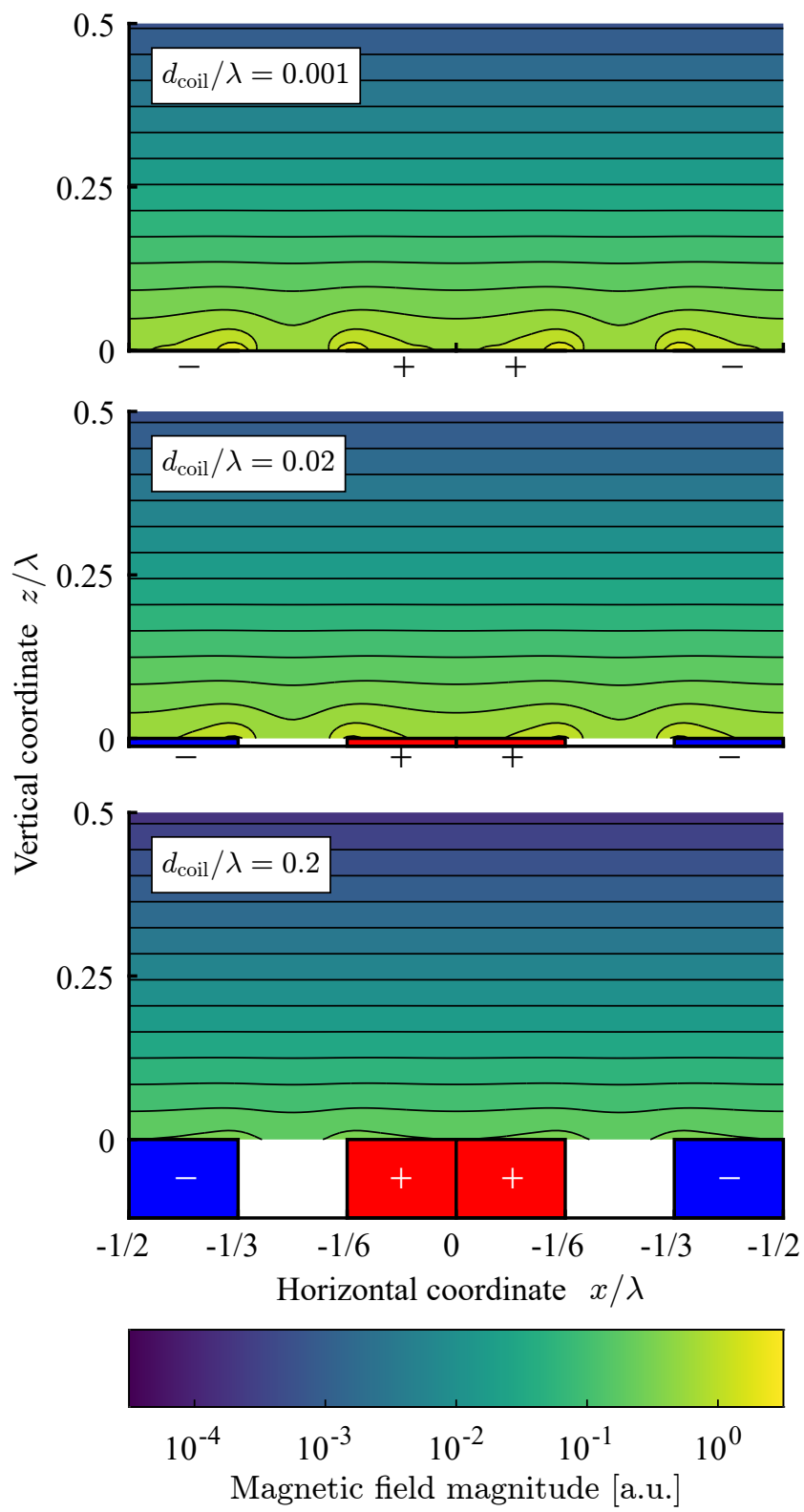

Figure 2.11: Magnetic field magnitude above configurations consisting of an infinite number of racetrack coils, for varying thicknesses of the winding pack (scaled to the periodicity $\lambda$ ). Two coils are plotted (each with a width $\lambda / 2$ ), and the polarity of the current is indicated by a red colour with a plus sign and by a blue colour with a minus sign. Thicker coils produce a smoother field, as the $K_{1}$ component is relatively large compared to the higher order components. 


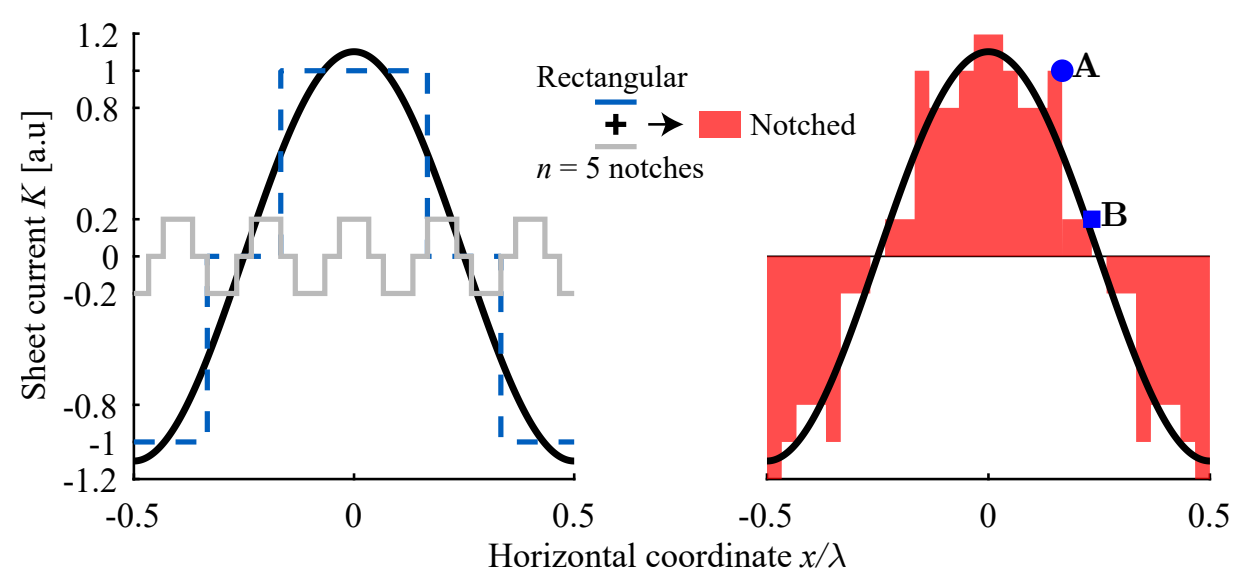

Figure 2.12: Rectangular (dashed blue) sheet current distribution with periodicity $\lambda$. This distribution is an approximation of the ideal (black) harmonic distribution. The addition of notches (grey) in the winding pack with period and amplitude of $1 / 5$ of the main gives the current configuration shown in red. Point $A$ indicates the peak field location for thin coils, point $B$ for thick coils (i.e. thickness $\lambda 6$ and up).

is possible. The notched layout utilizes regions where the engineering current density $J_{\mathrm{e}}$ is $20 \%$ higher than in the un-notched layout. The effect of this varying engineering current depends on the strategy adopted to realize the notching, since this can be achieved in multiple ways:

1. Use the same single conductor for the entire winding pack, with varying packing factors (by, for example, adding copper strands between windings). With this option $J_{\mathrm{e}}$ varies with the $x$-coordinate whereas the current density $J$ in the superconductor is constant;

2. Use a single type of conductor with the same packing factor throughout the winding pack, but use different current magnitudes to supply different sections. This way, $J_{\mathrm{e}}$ and $J$ both vary with the $x$-coordinate. A downside is a more complex electrical scheme.

3. Use conductors with different cross-sectional areas for different sections, possibly connected in series to the same power supply. $J_{\mathrm{e}}$ and $J$ both vary with the $x$-coordinate when one uses superconductors with the same fraction of superconductor in the windings.

The reason why the three different methods are mentioned is because the magnitude of the effect of a peak magnetic field difference between notched- and un- 
notched configurations depends on how $J$, and not necessarily $J_{\mathrm{e}}$, varies within the notched regions.

In Figure 2.13, the peak magnetic field in the different regions of the notched configuration are plotted as a function of winding pack thickness. The peak fields are scaled with the peak magnetic field of the simple rectangular configuration at the corresponding thicknesses. The peak field in the $0.8 J_{\mathrm{e}}$ region is not shown: its magnitude is always below that of the $1.2 J_{\mathrm{e}}$ region.

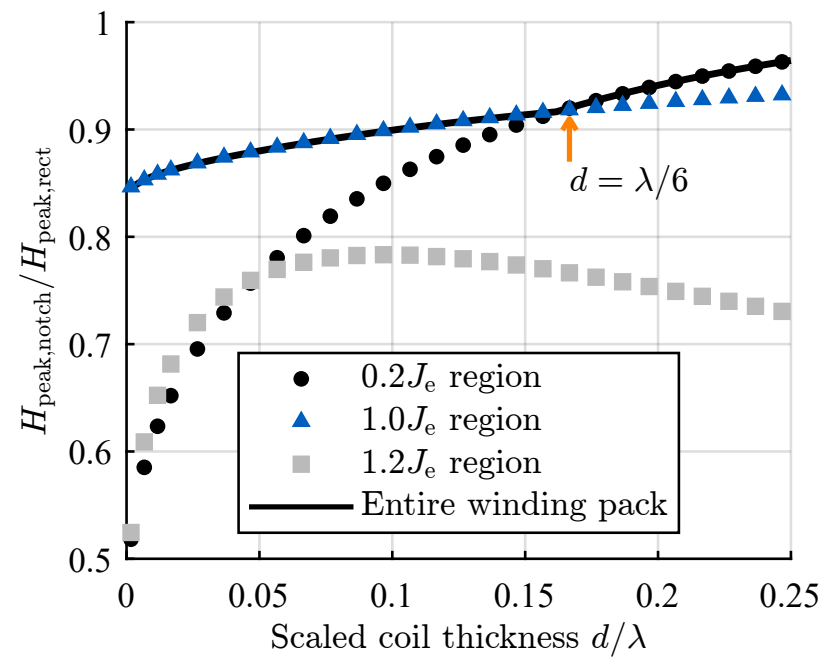

Figure 2.13: Peak magnetic field in different regions of the notched configuration relative to peak magnetic field in simple rectangular configuration, as a function of winding pack thickness. Data are both from the Fourier method (solid black line) and COMSOL simulations (markers).

For thin coils, the maximum magnetic field is located at the $1 J_{\mathrm{e}}$ section (point A in Figure 2.12). This maximum grows from $85 \%$ of the rectangular distribution peak magnetic field to $92 \%$ at $d_{\text {coil }}=\lambda / 6$. Here the peak field switches location to the $0.2 J_{\mathrm{e}}$ section (point $\mathrm{B}$ in Figure 2.12). If one winds the coils using the varying packing factor method, the current density in the conductor itself will be the same in the $1 J_{\mathrm{e}}$ and $0.2 J_{\mathrm{e}}$ sections and thus this shift of peak field will mean a reduced $J_{\mathrm{c}}$ compared to the other configurations. For thick coils however, the difference in performance with the simple rectangular profile is small anyhow.

The peak field magnitude in the $1.2 J_{\mathrm{e}}$ section increases sharply with increasing winding pack thickness, plateaus around $d_{\text {coil }}=\lambda / 10$, and then decreases. It will depend on the critical surface of the specific superconductor under consideration whether this $1.2 J_{\mathrm{e}}$ region or the $1 J_{\mathrm{e}}$ (or even the $0.2 J_{\mathrm{e}}$ ) region is the bottleneck in achieving the desired stability. 
An additional downside to the notched distribution arises when one moves to a full 3D consideration. Then the notched layout will have parts of the semicircular sections (i.e. the heads) of the racetrack coil that have a smaller inner radius than an un-notched coil. This will likely increase the peak magnetic field in the ends compared to a simple rectangular distribution. Reducing this effect may require flaring the ends to a bigger extend than one may deem necessary for simple rectangular racetracks, causing extra complexity to the already complicated configuration and increasing the required conductor volume.

Also, when one considers the effects of having a finite number of coils, the end-effects may be the dominant source of field deviations for some coil layouts, thus reducing the relative benefit obtained by using a more complex winding pack.

For our NbTi demonstrator magnet, we decided to use racetrack coils with a simple rectangular cross-section, since simplicity is valued above optimization for this first-of-a-kind magnet.

\subsection{Conclusion}

$\mathrm{M}$

AGNETIC density separation requires a magnetic field with a strong vertical gradient that changes with distance to the magnet but does not vary in the horizontal plane. Such a magnetic field can in principle only be generated by a pure harmonic sheet current with period $\lambda$. This period determines the decay rate of the exponentially decaying field magnitude. The obtainable separation resolution scales linearly with $\lambda$.

A practical magnet configuration that approximates this ideal current distribution consists of a planar array of racetrack coils with a characteristic leg width equal to one third the period $\lambda$.

Racetrack coils with a small thickness are inefficient as far as that the usable magnetic field gradient at the fluid bed relative to the peak magnetic field inn the coils is low. This can be explained by considering the Fourier components of the magnetic field. Also, the higher order Fourier components represent an unwanted ripple in the magnetic field profile. Their effect at the fluid bed is stronger for thin coils than for thick coils.

A further refinement of the current distribution can be achieved by a 'notched' current profile, which reduces field ripples close to the magnet. This refinement also reduces the peak field in the conductor compared to simple racetracks. This effect is larger for thin winding packs. The downside is the added winding complexity. For the NbTi demonstrator magnet under construction at the University of Twente, it was decided to use three racetrack coils with a simple rectangular cross-section.

The considerations in this work are applicable not just to MDS. They are valid 
for applications in general where a magnetic field gradient perpendicular to the magnet surface is required that changes with distance from the magnet and is constant in the parallel plane. 


\section{3 | Electromagnetic design}

In this chapter is described how the optimum layout of coils can be determined for superconducting magnets for use in Magnetic Density Separation. Also an estimate of the achievable vertical magnetic field gradient in the ferrofluid bed is presented.

Edge effects caused by the practical necessity of using a finite number of coils play a dual role. They cause a horizontal magnetic field gradient to appear and they change the vertical magnetic field gradient. The vertical magnetic field gradient profiles of various layouts of coils are compared to see which configuration performs best. To ensure that the coils are practical to produce, the analysis is restricted to flat racetrack coils. As far as the vertical magnetic field gradient is concerned, the specific shape of a racetrack coil has a larger influence than the number of coils.

The feed particles need to be pushed from the insertion point in the separation chamber to their collection points. One option to realise this is to use an MDS setup in which the plane of coils is inclined with respect to the horizontal plane. This tilt results in a horizontal magnetic force, which pushes feed particles through the fluid bed in a horizontal direction. A three-coils layout will be shown to provide the largest usable fluid bed depth for the system tilted over a wide range of angles. The demonstrator NbTi magnet is designed to be placed at an angle of $12^{\circ}$ and consists of three coils.

In the second part of the chapter the focus is on estimates of the achievable performance of superconducting MDS magnets by considering the quantity of superconductor used, as well as the choice of the superconducting material. The result is that a magnitude $20 \mathrm{~T} / \mathrm{m}$ vertical magnetic field gradient is possible when using NbTi conductor. Extrapolation of the demonstrator's mechanical design yields a potential performance of ReBCO and $\mathrm{Nb}_{3} S n$ magnets of around $43 \mathrm{~T} / \mathrm{m}$. If the mechanical design could be improved further, this vertical magnetic field gradient can increase to $60 \mathrm{~T} / \mathrm{m}$ for $\mathrm{Nb}_{3}$ Sn and to over $75 \mathrm{~T} / \mathrm{m}$ for ReBCO coils.

Finally, the attraction force between the ferrofluid and the magnet is calcu- 
lated. This force is important for two main reasons. The first is that the cryostat wall separating the ferrofluid from the vacuum needs to withstand the pressure of the ferrofluid pushing against it. This pressure is of comparable magnitude to the atmospheric pressure on the vacuum enclosure. The second reason is that the coils needs to be kept in position from moving toward the fluid.

\title{
3.1 Number- and type of racetrack coils
}

\begin{abstract}
S motivated in Chapter 2, Magnetic Density Separation (MDS) ideally reA quires a vertical magnetic field gradient that changes only with the distance perpendicular to the plane of the set of flat coils and is constant in the parallel direction [1]. Sheet current distributions on an infinite plane are of great help mathematically for initial considerations. These yielded the results presented in the previous chapter. It was found that a magnetic field with an exponentially decaying magnitude with distance from the magnet is the only magnetic field that meets the requirement perfectly, and that such a magnetic field is generated by a harmonic sheet current. This sheet current was then approximated using an infinite number of flat racetrack coils.
\end{abstract}

Of course, truncation to a finite size is always required in any practical magnet design, since it uses a finite number of coils. This reality has consequences for the magnetic field profile, with edge effects appearing. Here it is considered how such effects influence the design of magnets that aim to produce a strong vertical magnetic field gradient.

The focus in the first part of this chapter is on how the selection of the number of coils in a superconducting MDS magnet depends on the edge effects introduced by a finite number of coils. The motivation behind a three-coil design is explained by considering how the specifics of particle separation are affected by the shape and magnitude of the vertical- and horizontal magnetic field gradients. The same considerations can also be followed to find the optimum number of coils for future superconducting MDS systems.

First, the specific method used to move feed particles across the separation chamber, which shall be referred to as magnet-driven MDS, is presented. This is followed by different ways to approximate the ideal magnetic field shape using racetracks coils. There after, the smoothness of the vertical magnetic field gradient produced by various numbers- and types of coils is considered. It is shown that the vertical magnetic field gradient determines the best specific shape of the racetrack coil. Finally, the horizontal magnetic field gradient is considered. The impact of the horizontal field gradient depends on the method used to move feed particles across the separation chamber. The choice for magnet-driven MDS, in which the set of coils is placed at an angle to the horizontal, results in an optimum number 
of three coils, irrespective of the angle employed.

\subsubsection{Magnet-driven MDS}

There are two qualitatively different strategies to drive the feed particles from one side of the separation chamber to the other.

The first method introduces a flow in the ferrofluid that drags the particles along. Characteristic flow speeds range from 0.1 to $0.3 \mathrm{~m} / \mathrm{s}$ [2]. In such a fluiddriven system, the minimum number of coils follows from the chosen flow speed and from the requirement that the feed particles are given sufficient time to reach their equilibrium heights. This time can be calculated by solving the trajectories of the feed particles numerically.

The second method introduces a horizontal component of the magnetic force to push the particles from their insertion point in the fluid towards the separator blades. This horizontal component can be created by placing the plane of coils at an angle with respect to the horizontal plane [40]. We refer to such an tilted MDS system as magnet-driven.

Figure 3.1 shows a schematic where the magnet is rotated around the $y$-axis, introducing an angle $\alpha$ between the $x$ - and $x^{\prime}$-axes (or between $z$ and $z^{\prime}$ ). Since the ferrofluid pushes the feed particles away from the magnet in the $z^{\prime}$-direction, due to the fluid-magnet attraction, the force on a particle acquires a horizontal $x$-component when the magnet is tilted. Thus the particles do not rely on a fluid flow in order to arrive at the collection points. This allows for a better separation resolution since turbulence is reduced [40].

Since the demonstrator is magnet-driven, the focus in this section is on the tilted configuration. This is especially important in the discussion of the horizontal component of the magnetic field gradient. All considerations regarding the vertical component are also relevant for fluid-driven systems. To answer the question which practical coil configuration is best suited for a magnet-driven MDS, several coil layouts are introduced and compared.

\subsubsection{Potential coil layouts for MDS}

As derived in Chapter 2, the ideal magnetic field configuration $H\left(x^{\prime}, y, z^{\prime}\right)$ for MDS has a gradient of its magnitude, $\mu_{0} \nabla H$, that only changes with distance $z^{\prime}$ from the magnet, and remains constant parallel to it in the $x^{\prime} y$-plane. Such a magnetic field can be generated by a periodic sheet current $K$ on an infinite $\left(x^{\prime}, y\right)$ plane of the form (Figure 3.2b)

$$
\boldsymbol{K}\left(x^{\prime}\right)=K_{0} \cos \left(\frac{2 \pi}{\lambda} x^{\prime}\right) \hat{\boldsymbol{y}}
$$




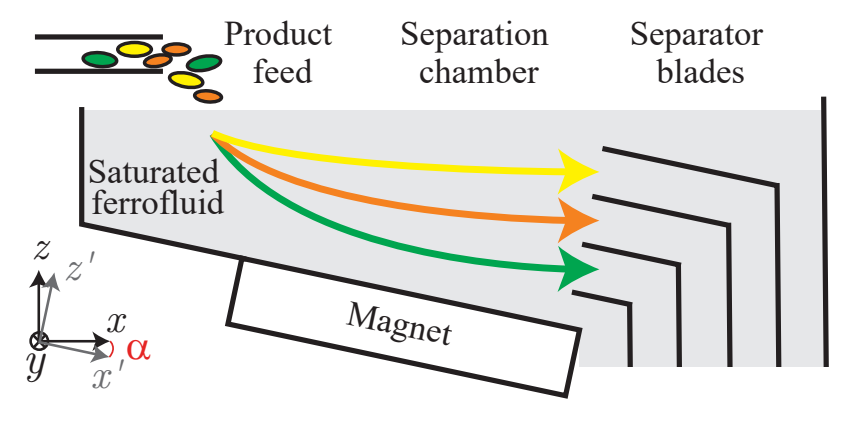

Figure 3.1: Schematic of a magnet-driven MDS system. Non-magnetic feed particles are immersed in a ferrofluid that is attracted to a magnet. When the magnet is tilted at an angle $\alpha$ with respect to the horizontal, the combination of vertical forces on the feed particles (gravity, buoyancy and effective repulsion by the magnet) dictates their equilibrium height, whereas the horizontal component of the magnetic repulsion pushes them towards the separator blades. After separation, the particles are collected by a conveyor belt that runs out of the sketched plane. Also indicated are the coordinate systems used in this chapter: $z$ is the vertical direction while $z^{\prime}$ indicates the normal distance to the magnet surface.

where $K_{0}$ is the magnitude of the sheet current and $\lambda$ its periodicity. The resulting magnetic field above ${ }^{1}$ the sheet has a magnitude $H$ that decays exponentially with the distance $z^{\prime}$ from the sheet but does not depend on $x^{\prime}$ or $y$ :

$$
H\left(z^{\prime}\right)=\frac{K_{0}}{2} \exp \left(-\frac{2 \pi}{\lambda} z^{\prime}\right) .
$$

Note how the periodicity of the current distribution $\lambda$ dictates the exponential decay length of the magnetic field magnitude. For the demonstrator, $\lambda$ is chosen to be $600 \mathrm{~mm}$. The difference in equilibrium height between two particles with different mass density, and hence the separation resolution, scales linearly with $\lambda$. The value of $600 \mathrm{~mm}$ corresponds to the desired resolution for the separation of electronic material.

\subsubsection{Practical layouts of racetrack coils.}

A practical approximation of such an idealized harmonic current distribution can be created with racetrack coils, placed in such a way that the current distribution

\footnotetext{
${ }^{1}$ We assume that the feed particles have a higher mass density than the ferrofluid. Separation of feed streams with a lower mass density, e.g. certain plastics, is possible by placing the magnet above the fluid, pushing the particles into it [2].
} 


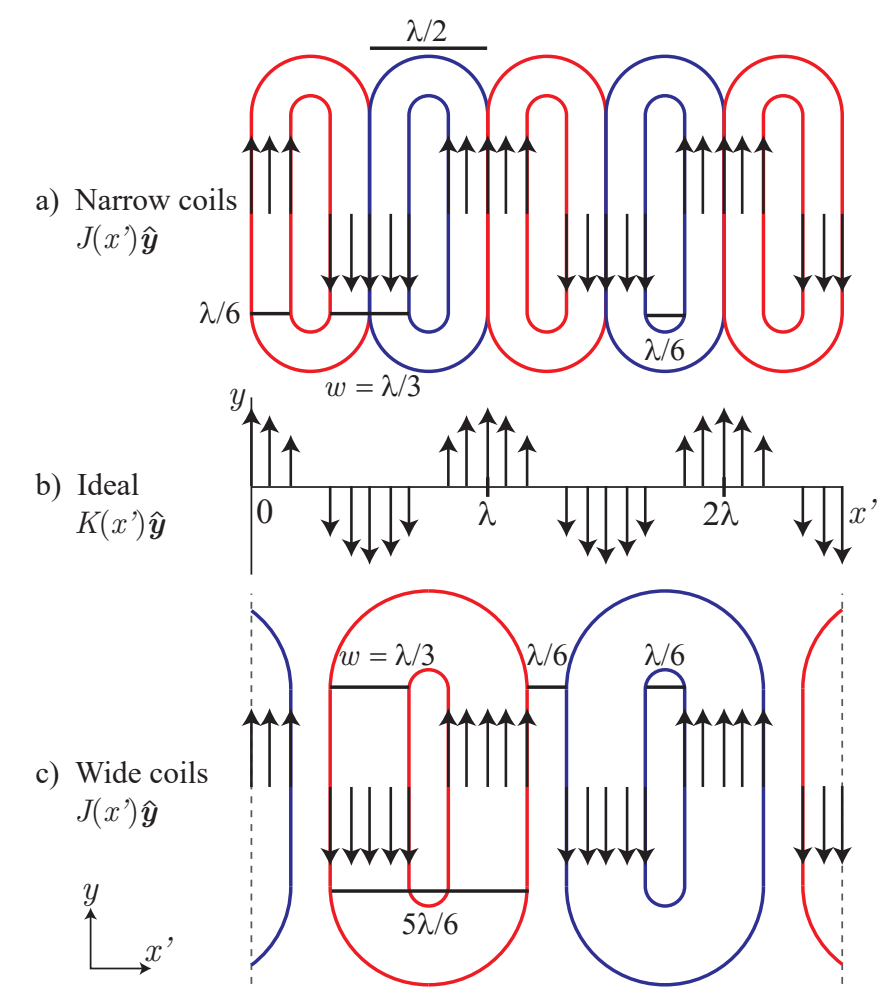

Figure 3.2: Top view of coil layouts, showing the ideal sheet current distribution $K$ for MDS (b) and two different methods (a and c) to approximate this sheet current with racetrack coils with rectangular cross-section that carry a uniform current density $J$. The leg width $w$ of the rectangular sections ideally is set to $\lambda / 3$. The first method (a) uses coils with a leg width $\lambda / 6$ and total width $\lambda / 2$ positioned directly adjacent to each other. The current direction (black arrows) in touching sections is the same. The second method (c) uses coils with a leg width $\lambda / 3$ and a coil width $5 \lambda / 6$, spacing them $\lambda / 6$ apart. Note that the layout of the semi-circular end sections can be optimized by segmenting them, in order to reduce the peak magnetic field in the conductor.

varies stepwise with the $x^{\prime}$-coordinate. Preferably the width $w$ of the racetracks' straight sections is set to $\lambda / 3$ (Figure 3.2), as explained in Chapter 2. In this way the most dominant, unwanted, harmonic is suppressed. There are two straightforward ways to realize such a current distribution:

- Racetracks coils, each with a total width of $\lambda / 2$ and a 'leg' width $\lambda / 6$, are positioned next to each other in the $x^{\prime}$-direction (Figure 3.2a). The 
current in adjacent legs flows in the same direction. We refer to this type of racetrack as a narrow-coil;

- Racetracks coils with a total width $5 \lambda / 6$ and a leg width $\lambda / 3$ are positioned with periodicity of $\lambda$ in the $x^{\prime}$-direction (Figure 3.2c). This type of coil is referred to as a wide-coil. A downside of using wide coils is that more material is needed to make the semi-circular end sections. Also, the extra width of a coil for a given value of $\lambda$ can present more difficulties in coil winding.

In the remainder of this section we focus on the magnetic field above the straight sections of the racetrack coils and do not consider the semi-circular parts. This is justified because the fluid bed is located above the straight sections of the coils.

The main questions addressed in this section are the optimum number of coils for an (angled) MDS magnet and whether the coils have to be of the wide- or narrow variant. The questions are answered by focusing on two quantities:

- the vertical component of the gradient of the magnetic field magnitude, $\partial H / \partial z$, which determines the equilibrium height of feed particles. Its variation with the horizontal coordinate $x$ influences the smoothness of particle trajectories;

- the horizontal component of this gradient in the direction of movement, $\partial H / \partial x$. This component affects the horizontal movement of feed particles relative to the fluid, speeding them up or slowing them down.

With the different coil layouts introduced, in Sections 3.1.2.2 and 3.1.2.3 the focus is on the 2D magnetic field profiles in the $x^{\prime} z^{\prime}$-cross-sections (Figure 3.1) that coincide with the symmetry plane of the coils in the $y$-direction (Figure 3.2). After determining the best-performing configuration, 3D simulation results are presented in Section 3.1.3 to argue that this $x^{\prime} z^{\prime}$-plane is representative for the entire fluid bed above the straight sections of the coils. Obviously the 2D simulations do not provide details on the component of the gradient in the $y$-direction.

For the narrow-coil family, configurations made up from one to five coils are investigated. For the wide coils, up to three coils are considered. The eight different configurations are shown in Figure 3.3, along with the magnetic field profiles above the coils that they produce.

The truncation of the periodic current distribution in the $x^{\prime}$-direction to a finite number of (half-) periods changes the magnetic field compared to the ideal solution (Eq. 3.2). Maybe the most striking difference is the appearance, for some configurations, of local zeroes in the magnetic field. In the vertical direction such 

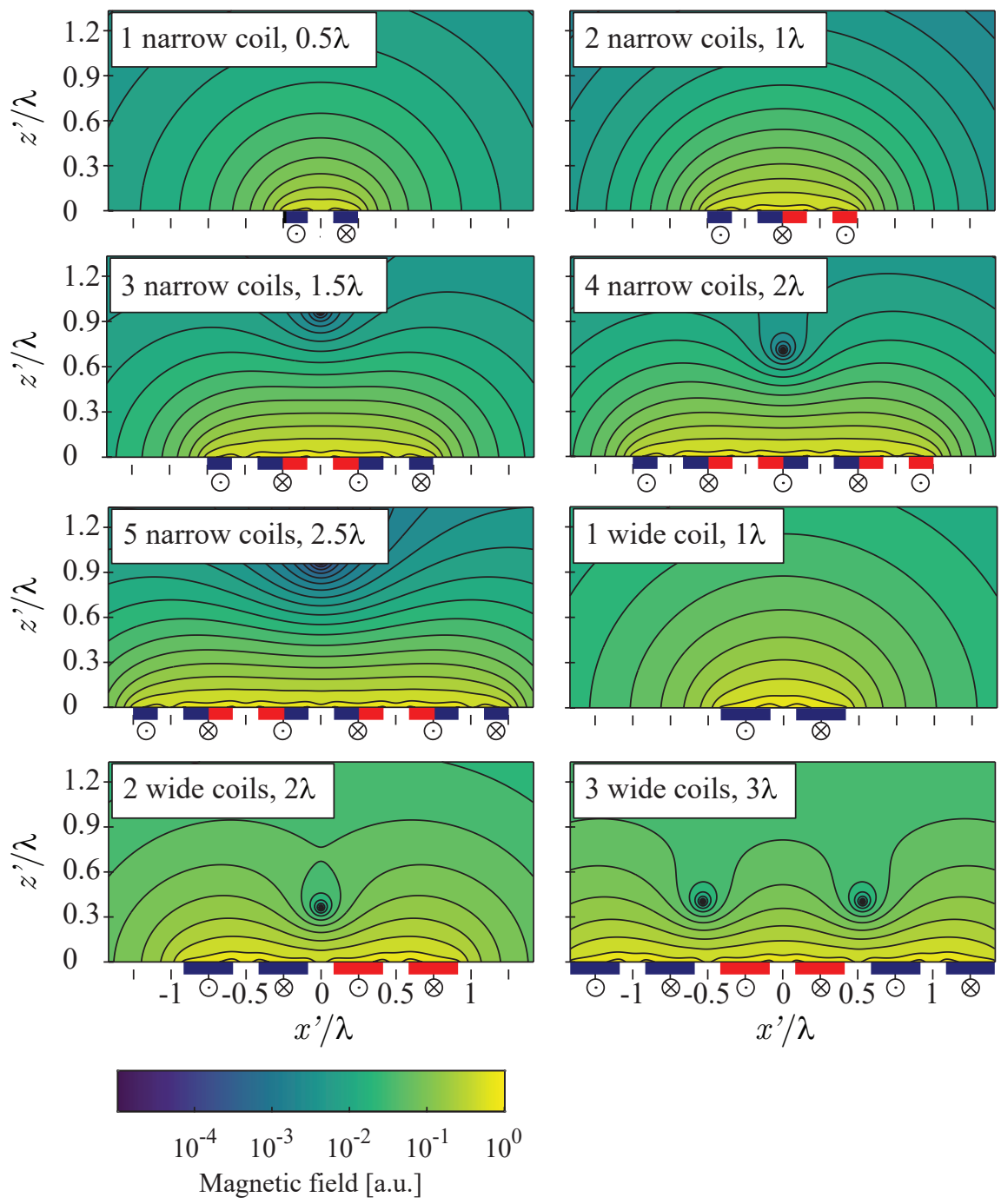

Figure 3.3: Logarithm of the magnetic field magnitude $(\log H)$ above the surface of magnet systems consisting of varying numbers and types of coils. $x^{\prime} z^{\prime}$-crosssections at the mid-plane in the y-direction are shown. The racetrack coils are indicated alternately in red and blue. The small circles below the winding packs indicate the current direction (in the $\pm y$-direction). The origin of the $z^{\prime}$-axis is placed at the top surface of the coils. The magnetic field strength is normalized with the maximum value in the fluid bed across all considered configurations. 
nodes tend to compress different-density feed particles together whereas in the horizontal direction they slow particles down. Both effects need to be minimized.

To compare the various configurations, we first consider the shape of the vertical magnetic field gradient.

\subsubsection{Variation in vertical magnetic field gradient}

In order to determine the optimal coil configuration for MDS, it is key to have a relevant criterion for magnetic field "quality". Ideally the gradient of the magnetic field magnitude, $\nabla H$ only has a vertical component that decays with the distance to the magnet. Variation of the vertical magnetic field gradient in the horizontal plane is to be suppressed as it causes the particle trajectory to 'wiggle' due to a changing equilibrium height, which may lead to incomplete separation or even re-mixing. The demand for a ripple-free vertical-gradient can be expressed as ${ }^{2}$

$$
\frac{\partial^{2} H}{\partial x^{\prime} \partial z^{\prime}}=0
$$

Essentially, different coil configurations can be compared by assessing how much they deviate from this condition. First a rough qualitative analysis is made of how well different coil configurations meet criterion (3.3). Then, for a more detailed comparison, a quantitative performance metric is formulated.

In Figure 3.4, contour lines of the vertical magnetic gradient $\left|\nabla_{z} H\right|$ are plotted for the eight configurations that were also presented in Figure 3.3. The lines can also be interpreted as the equilibrium heights of feed particles with different mass densities. Around the zero-field nodes, rings can be observed where $\nabla_{z} H=$ 0 . At these locations, different-density particles will be pushed together, which is unwanted. On first sight, the three-, four- and five narrow-coil layouts seem promising, since their contour lines look relatively flat and no zero-field nodes appear in the fluid bed.

To make this conclusion more quantitative, we consider a section of the fluid bed with a rectangular shape bound by $\left[z_{1}^{\prime}, z_{2}^{\prime}\right]$ and $\left[x_{1}^{\prime}, x_{2}^{\prime}\right]$. As a performance metric for fluctuations in the vertical magnetic gradient at a given distance $z^{\prime}$ above the magnet, the relative standard deviation $R S D$ is used. It is the standard

\footnotetext{
${ }^{2}$ The other requirement, $\frac{\partial^{2} H}{\partial y \partial z^{\prime}}=0$, is automatically fulfilled because the $y$-symmetry plane is considered.
} 

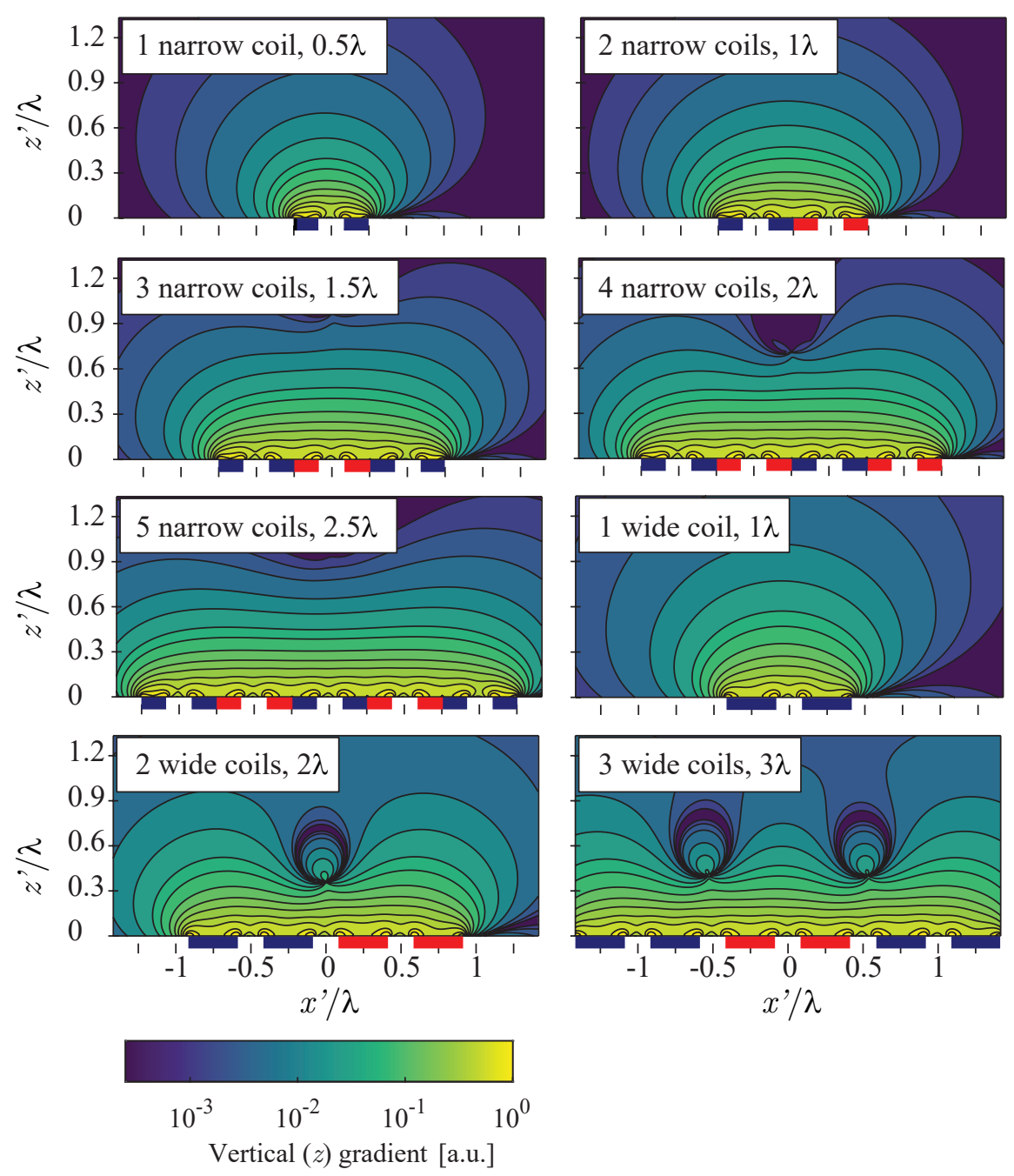

Figure 3.4: Logarithm of the magnitude of the vertical (z) magnetic field gradient, $\log \left|\nabla_{z} H\right|$, above the surface of the planar set of coils. Like Figure 3.3, the plots show the $x^{\prime} z^{\prime}$-cross-section at the $y$-symmetry plane. The coils are indicated in red and blue. The magnetic field gradient is normalized with its maximum value encountered across all considered configurations. The asymmetry in the $x^{\prime}$-coordinate is due to the $\alpha=12^{\circ}$ angle between the $z$-and $z^{\prime}$-axes (Figure $3.2)$. 
deviation of $\left|\nabla_{z} H\right|$ divided by its mean value in the $x^{\prime}$-range of interest $\left[x_{1}^{\prime}, x_{2}^{\prime}\right]$

$$
\begin{aligned}
& R S D\left(\left[x_{1}^{\prime}, x_{2}^{\prime}\right], z^{\prime}\right)=\frac{\sigma\left(\left[x_{1}^{\prime}, x_{2}^{\prime}\right], z^{\prime}\right)}{|\mu|\left(\left[x_{1}^{\prime}, x_{2}^{\prime}\right], z^{\prime}\right)} \\
& =\frac{\left[\frac{1}{x_{2}^{\prime}-x_{1}^{\prime}} \int_{x_{1}^{\prime}}^{x_{2}^{\prime}}\left(\nabla_{z} H\left(x^{\prime}, z^{\prime}\right) \mathrm{d} x^{\prime}-\mu\left(\left[x_{1}^{\prime}, x_{2}^{\prime}\right], z^{\prime}\right)\right)^{2} \mathrm{~d} x^{\prime}\right]^{0.5}}{\left|\mu\left(\left[x_{1}^{\prime}, x_{2}^{\prime}\right], z^{\prime}\right)\right|},
\end{aligned}
$$

where $\mu\left(\left[x_{1}^{\prime}, x_{2}^{\prime}\right], z^{\prime}\right)$ is the mean of the vertical field gradient $\nabla_{z} H$ at the height $z^{\prime}$, evaluated over the same $x^{\prime}$-range

$$
\mu\left(\left[x_{1}^{\prime}, x_{2}^{\prime}\right], z^{\prime}\right)=\frac{1}{x_{2}^{\prime}-x_{1}^{\prime}} \int_{x_{1}^{\prime}}^{x_{2}^{\prime}} \nabla_{z} H\left(x^{\prime}, z^{\prime}\right) \mathrm{d} x^{\prime} .
$$

The RSD value is shown in Figure 3.5 as a function of the normalized distance $z^{\prime} / \lambda$ to the surface of the coils. Configurations with two or three wide coils show large variations at distances $z^{\prime} / \lambda \gtrsim 0.3$, due to the presence of zero-field nodes in this area, as also obvious in Figures 3.3 and 3.4.

All configurations show large variations near the coil surface $\left(z^{\prime} / \lambda \lesssim 0.05\right)$. These are a direct consequence of the discrete "steps" in the current distribution $J$ of the racetrack system as opposed to the smooth harmonic ideal current distribution $K$ (Figure 3.2), as explained in Section 2.3. Note that a minimized fluid-coil distance is desired, since for a given number of ampere-turns this yields a higher vertical magnetic field gradient in the fluid bed and thus allows to separate heavier feed streams or, as discussed in the introduction, to use more dilute ferrofluids.

The impact of the variations near the coils on the separation performance depends on the coil-to-fluid distance. For the demonstrator, with $\lambda=600 \mathrm{~mm}$, this area with large fluctuations is situated within the first $30 \mathrm{~mm}$ from the coils, i.e. inside the cryostat (the distance between the coils and the fluid is $50 \mathrm{~mm}$ in this system). However, if one aims to use this region for the separation process (for example, when one manages to maintain the same coil-to-fluid distance with a larger- $\lambda$ magnet) it can be advantageous to use 'notched'-coils with a more complex shape that more closely resembles the ideal current distribution as explained in Section 2.3.

At intermediate distances from the coil surface $\left(0.05 \lesssim z^{\prime} / \lambda \lesssim 0.6\right)$, Figure 3.5 shows how for the narrow-coil layouts the deviations do not differ much from one configuration to the other. Narrowing the considered range $\left[x_{1}^{\prime}, x_{2}^{\prime}\right]$ results in lower RSD values, since edge effects then play a smaller role, and thus in a downward shift of all curves. However, the same qualitative behaviour remains. Thus based on the 'quality' of the vertical field gradient, we can draw two conclusions: (1) The wide-coil family is not effective if the useful fluid bed depth is 


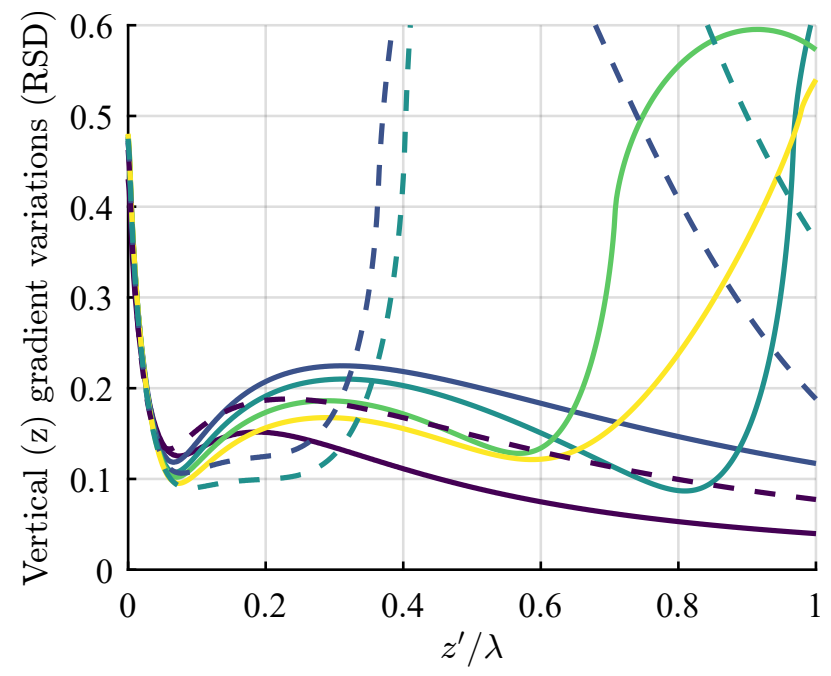

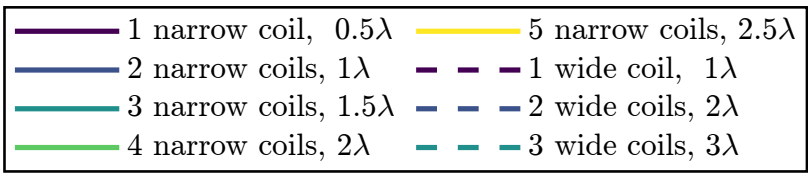

Figure 3.5: Normalized vertical z-gradient variation, as defined by Eq. 3.4b, as a function of distance $z^{\prime}$ from the top-surface of the magnet. The fluctuations in vertical magnetic field gradient at each height are evaluated using a horizontal coordinate between the left-most and the right-most $x^{\prime}$-coordinate of the coils. The winding pack thickness for these examples is $\lambda / 12$.

to be larger than $\lambda / 4$; and (2) in principle any reasonable number of narrow coils may be used. To determine an optimum number of narrow coils, the horizontal component of the field gradient needs to be considered as well. This is the focus of the next section.

\subsubsection{Effect of tilting the plane of coils}

For the demonstrator magnet a tilt angle $\alpha$ of $12^{\circ}$ was selected (Figure 3.1). If $\alpha$ is too large, the horizontal component of the force on the feed particles is too high, so that they have not arrived yet at their vertical equilibrium height by the time they reach the separator blades [40]. Also, a too fast travel speed may reduce the separation resolution due to fluid-dynamical lift forces on asymmetric feed particles. On the other hand, when $\alpha$ is too small, the MDS throughput is suboptimal since the particles' speed in the $x$-direction is smaller. The choice for a $12^{\circ}$ angle is based on experience of the end-user of the PM systems. Preferably 
the angle is between $5^{\circ}$ and $20^{\circ}$ for practical applications [40].

Another important issue is whether the horizontal component of the magnetic field gradient everywhere points in the correct direction in the fluid bed. This will determine whether all feed particles get pushed all the way across the separation chamber to the separator blades.

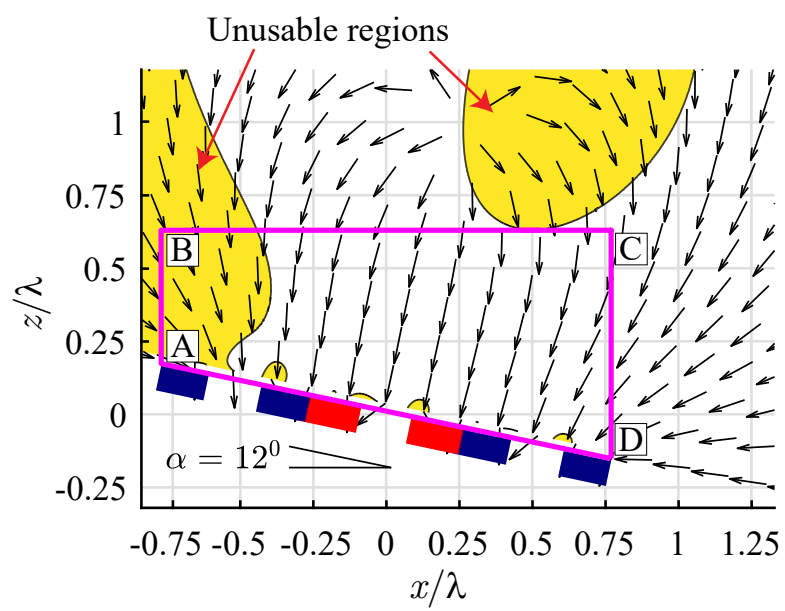

Figure 3.6: Direction of the gradient in the magnetic field magnitude (black arrows) above a tilted three-coil magnet (red and blue rectangles). Areas where the gradient has a positive $x$-component are indicated in yellow. These areas are unusable for MDS, since feed particles get pushed in the wrong negative $x$-direction there. This particular example shows coils tilted at $12^{\circ}$, but qualitatively similar observations can be made for all magnet-driven systems.

Figure 3.6 shows the direction of the magnetic field gradient in the ferrofluid above a three-coil system that is tilted at $12^{\circ}$. Areas where the $x$-component of the gradient is positive provide difficulties for the particle flow, since the magnetic force on the ferrofluid effectively pushes the feed particles in the opposite direction. Unlike in a fluid-driven system, the fluid does not drag the particles across these regions and thus these areas are unusable. This dictates a second demand on the magnetic field in the separation chamber, in addition to Eq. 3.3, namely:

$$
\frac{\partial H}{\partial x}<0 .
$$

The regions that do not meet this requirement are highlighted in Figure 3.6. The left-most coloured region limits where the feed particles can be fed into the fluid bed. The coloured region more to the right limits the usable fluid bed depth, and 
thus the separation performance for feed products with lower mass density. To compare this usable depth for different tilt angles and different numbers of coils, we define a vertical distance $A B$ between the top of the tilted magnet and the problem region, and $D C$ between the right-most location of the coils and the problem area. These distances are also indicated in Figure 3.6.
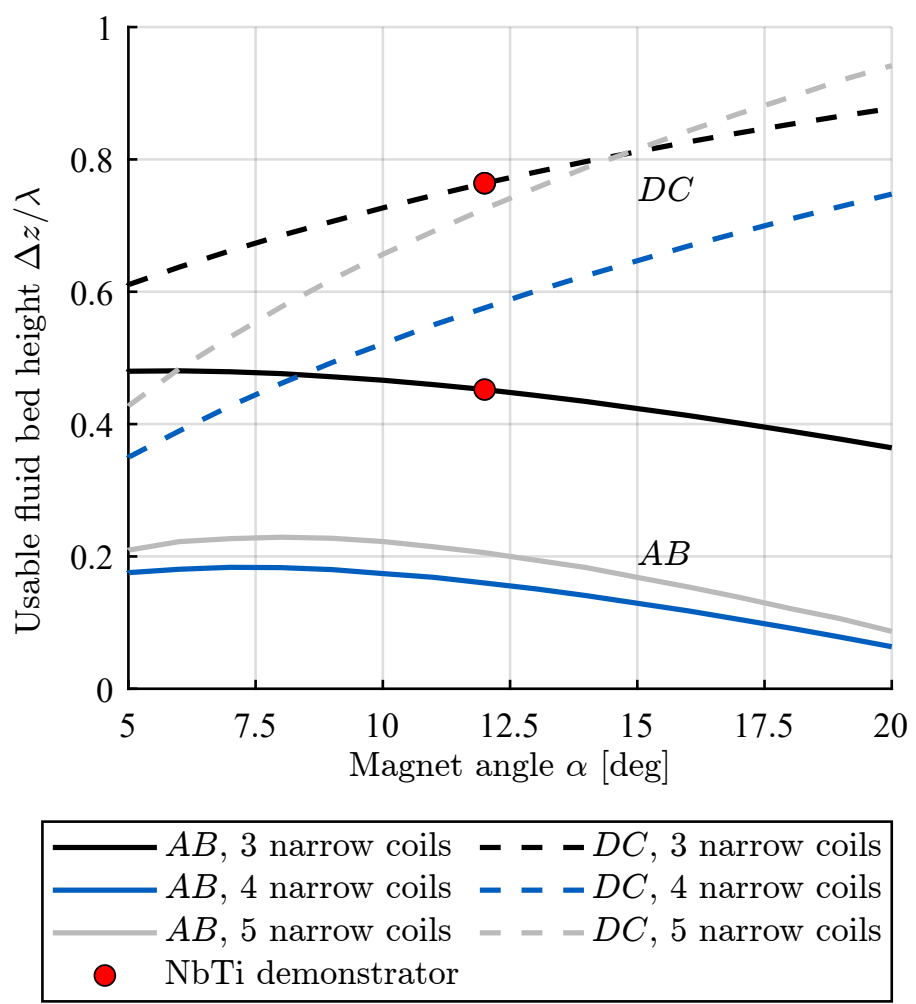

Figure 3.7: Usable vertical distance between the surface of the coils and the fluid surface, as defined in Figure 3.6, plotted against the tilt angle $\alpha$. Different colours correspond to different coil numbers, all of the narrow-coil family (Figure 3.2). Solid lines denote the vertical distance $A B$ between the left side of the coils and the unusable area. Dashed lines denote the vertical distance DC between the extraction side of the magnet and the problem area. To determine the actual useful fluid depth, one needs to subtract the distance between the coils and the cryostat surface, which is roughly $50 \mathrm{~mm}$ (or V12) for the NbTi three-coil magnet design (indicated with the red symbols).

$A B$ and $D C$ are shown as a function of the tilt angle $\alpha$ in Figure 3.7, for three to five narrow coils. $D C$ is indicative of the obtainable separation resolution, since 


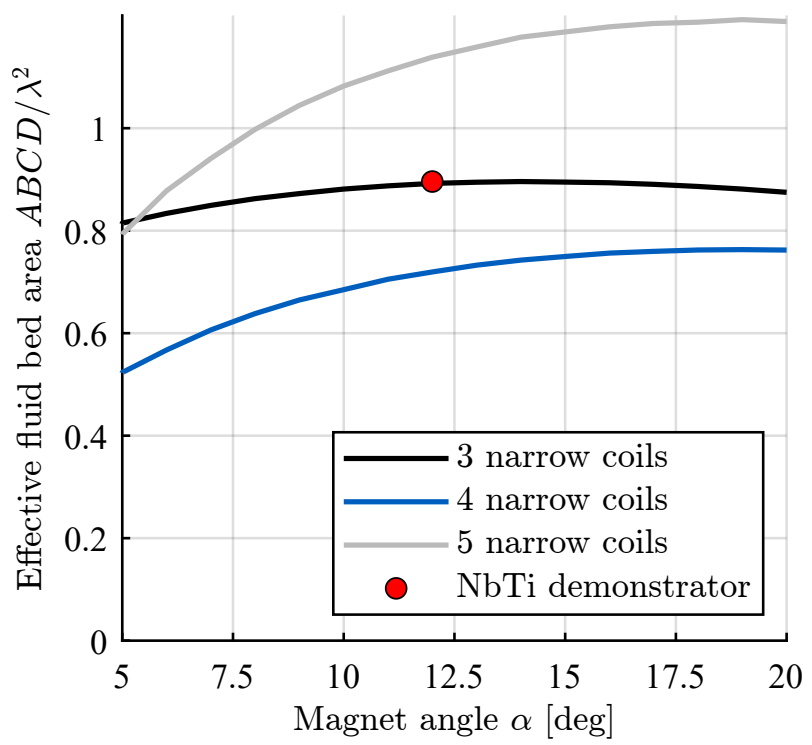

Figure 3.8: Scaled effective fluid bed area $A B C D / \lambda^{2}$, as defined in Figure 3.6, versus the magnet tilt angle $\alpha$. Different colours correspond to layouts with different numbers of coils, all of the narrow family.

it determines the range over which separator blades (Figure 3.1) can be spaced. A low $A B$ value is not useful since it implies that feed particles with a relatively low mass density are pushed to the ferrofluid surface after being inserted in the fluid bath, and only start to separate once they have been pushed further along the fluid bed. Thus a large value of both $A B$ and $D C$ is desired. Figure 3.7 clearly illustrates how the 3-coil layout provides the largest usable depth of the fluid bed for a wide range of tilt angles. This is the main reason that three coils placed at a $12^{\circ}$ angle were selected for the final layout of the $\mathrm{NbTi}$ demonstrator.

Not only the vertical distance is of interest, but also the effective fluid bed volume. In this respect, the area enclosed by $A B C D$ is taken as a rough figureof-merit. A large value of $A B C D$-area is beneficial as it minimizes the effect of interactions between feed particles, due to the extra available space. The result is an enhanced separation resolution and throughput.

$A B C D$-area as a function of the tilt angle is shown in Figure 3.8. The threecoil layout provides a larger separation volume than the four-coils one. The fivecoil layout performs similar to the three-coil one at lower $\alpha$-values. At larger angles the five-coil configuration provides a larger separation volume, though always below a 5/3 ratio compared to the three-coil one. This means that the 3-coil system provides the largest separation volume per coil, which can be an important 
consideration for MDS systems in which the coils contribute a significant factor to the expenditure over the lifetime of the system. Subtracting the part of this area inside the cryostat or subtracting the left-most problem region in Figure 3.6 does not change these conclusions.

The wide-coil configurations are omitted from this discussion since they generate regions with a positive horizontal component of the magnetic field gradient too close to the magnet to make a magnet-driven strategy possible.

\subsubsection{D analysis}

In this section is presented a full 3D magnetic field calculation of the threenarrow-coil solution made with COMSOL to verify that the conclusions drawn above based on 2D calculations of the magnetic field profile are still valid when one approaches the semi-circular "bends" at both ends of the racetrack coils (Figure 3.2). Figure 3.9 shows contour lines of the vertical $z$-component of the gradient in three $x^{\prime} z^{\prime}$-planes corresponding to different $y$-values. The length of the straight sections of the racetrack coils is set to $1 \mathrm{~m}$. Note that this length determines the throughput of the MDS process. The plane at $y=0$ corresponds to the horizontal symmetry plane in Figure 3.2, while the $y=0.5 \mathrm{~m}$ plane shows the transition between the racetrack coils' straight and semi-circular sections. $\lambda$ is set to $600 \mathrm{~mm}$ as in the demonstrator.

The contour lines move closer to the coil surface for $y$-values further from the center of the coils, but these deviations are small enough $(\Delta z \lesssim 10 \%)$ to be correctable by slightly shaping the separator blades.

Another option would be to bend the winding packs themselves so that the distance between the ferrofluid and the coils is slightly higher near the middle $(y=0)$, which would also reduce particle motion in the $y$-direction. However, for this first-of-its-kind demonstrator the focus is on simplicity and ease of coil winding, so that shaping the magnetic field by bending the coils was omitted. Also, this approach is impractical for superconductors in tape-form whose performance suffers under so-called 'hard-bending' [41].

As for the variation along the $y$-direction of the $x$-component of the gradient, the location and shape of the unusable regions shown in Figure 3.6 do not change significantly when coming close to the racetrack coils' semicircular sections.

Another aspect of interest is the ratio $\frac{\partial H}{\partial y} / \frac{\partial H}{\partial x}$ between the two horizontal components of the magnetic field gradient. This ratio determines how much particles move along the $y$-direction towards the side of the fluid tank while travelling through the fluid bed in the $x$-direction and is shown in Figure 3.10. The relative importance of the $y$-component gets stronger with increasing distance to the coil surface. 


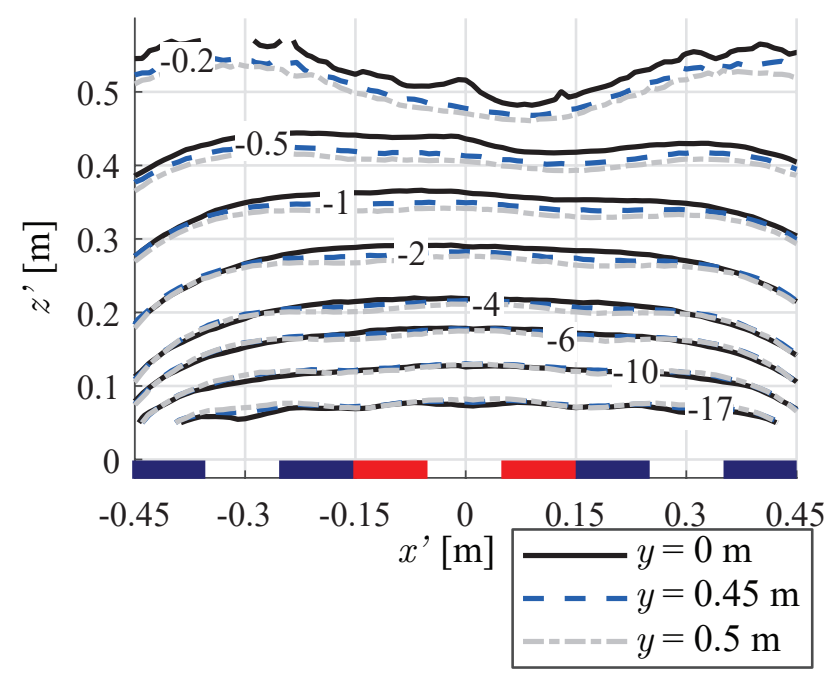

Figure 3.9: Contour lines of the vertical $z$-component of the gradient $\mu_{0} \nabla_{z} H$, in $[\mathrm{T} / \mathrm{m}]$, for three vertical $x^{\prime} z^{\prime}$-planes located at different $y$-coordinates (indicated with different line styles). The example shown is for the $12^{\circ}$ angled NbTi demonstrator comprising three coils (shown in blue and red) with a winding pack thickness of $50 \mathrm{~mm}$. The length of the straight sections of the racetrack coils is $1 \mathrm{~m}$. The coordinate system used is defined in Figure 3.1 and $z^{\prime}=0$ corresponds to the top surface of the coils. Each coil has 2230 turns and carries a current of $300 \mathrm{~A}$.

Figure 3.10 also shows a sketch of the coils. The bent coils' heads are split into two sections, which reduces the peak magnetic field in the bends from $119 \%$ down to $107 \%$ of the magnetic field in the straight sections. In Figure 3.9 the effect of this modification is taken into account.

In principle, in a fluid-driven MDS system the ferrofluid can flow in either the $x$ - or $y$-direction. However, the 3D simulations show that with a magnetdriven system tilted around the $x$-axis (Figure 3.2) the resulting component of the gradient is insufficient to push particles to the collections points. Note that the feed particles are driven in the $x$-direction (Figure 3.2), even if in principle for the fluid-driven MDS type a fluid flow may be allowed either in the $x$ - or the $y$-direction. More precisely stated, large ineffective areas appear with positive $y$-component of the magnetic field gradient. 

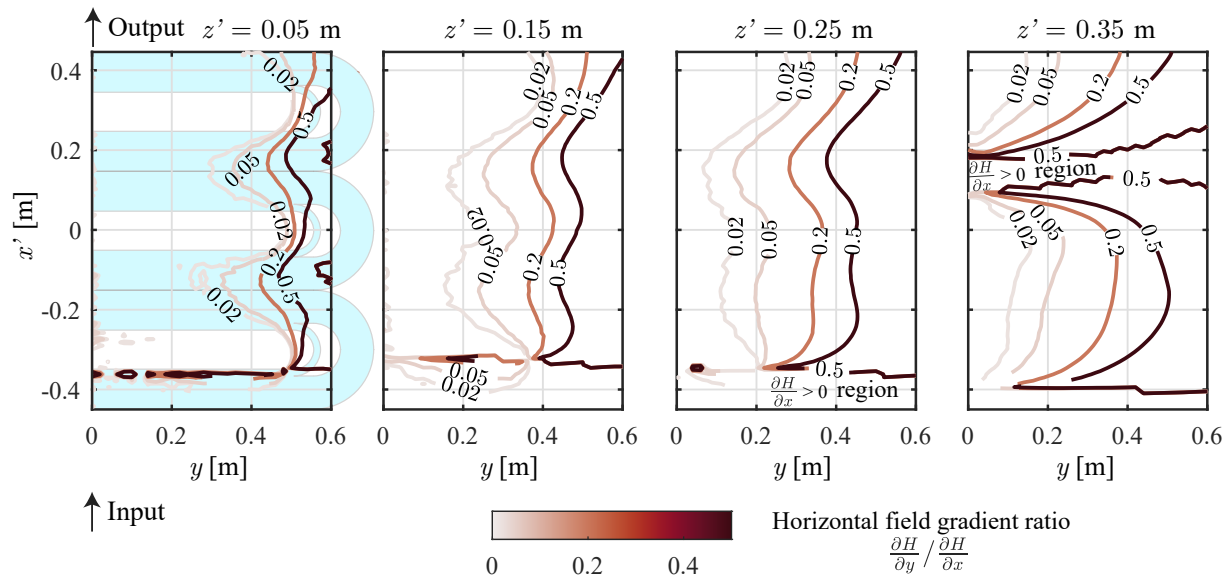

Horizontal field gradient ratio $\frac{\partial H}{\partial y} / \frac{\partial H}{\partial x}$

Figure 3.10: Contour lines of the ratio between the horizontal components of the magnetic field gradient, evaluated in $x^{\prime} y$-planes at different distances $z^{\prime}$ to the coil surface. The coils are included in the left-most subplot. The y-component of $\nabla H$ should be minimal so as to minimize movement of particles towards the side-walls. The relative importance of the unwanted y-gradient increases with increasing distance to the magnet. Feed particles have to be inserted into the ferrofluid at $x^{\prime}$-values larger than $-0.4 \mathrm{~m}$, due to the presence of a positive $x$ gradient region, as discussed in the context of Figure 3.6. The other problem region is visible at a distance $z^{\prime}$ of $0.35 \mathrm{~m}$ (the rightmost subplot). 


\subsubsection{Winding pack geometry and composition}

In the previous discussion, the winding pack thickness was set to $50 \mathrm{~mm}$. This corresponds to the demonstrator, whose key properties are summarized in Table 3.1. The system consists of three coils, each with a width of $300 \mathrm{~mm}$, and a length of the straight sections of $1 \mathrm{~m}$. The splitting of the coil heads to reduce the peak magnetic field extends the length of the coils by $100 \mathrm{~mm}$, resulting in a total coil length of $1.4 \mathrm{~m}$ in the $y$-direction. This segregation of the coil heads is detailed in Section 3.2.2. The coil geometry as discussed allows to generate a 2.0 T magnetic field at the bottom of the fluid bed using NbTi conductor. This performance estimate is elaborated further in the next section of this chapter.

Table 3.1: Main parameters of the NbTi demonstrator magnet relevant for magnetic density separation. The narrow coil variant was introduced in the context of Figure 3.2.

\begin{tabular}{llll} 
Parameter & Symbol & Value & Unit \\
\hline Magnetic field at fluid bed & $\mu_{0} H_{\text {fluid }}$ & 2.0 & $\mathrm{~T}$ \\
Coil-fluid distance & & 50 & $\mathrm{~mm}$ \\
Characteristic periodicity & $\lambda$ & 600 & $\mathrm{~mm}$ \\
Magnet tilt & $\alpha$ & 12 & degrees \\
Racetrack coil type & & narrow & \\
Number of coils & & 3 & \\
Coil width & $\lambda / 2$ & 300 & $\mathrm{~mm}$ \\
Winding pack thickness & $d_{\text {coil }}$ & 50 & $\mathrm{~mm}$ \\
Coil straight section length & $L_{y}$ & 1000 & $\mathrm{~mm}$
\end{tabular}

The coils themselves are composites consisting of materials with very different mechanical, electrical and thermal properties. An illustration of the major coil components is presented in Figure 3.11.

Each coil consists of an AISI304 stainless steel yoke around which the wire is wound using a wet winding technology. The turns are glued together using Stycast 2850FT blue epoxy resin. The steel stainless yoke has a Teflon coating to prevent adhesion of the turns to the yoke. The justification for this design is given in Chapter 4. The winding procedure itself is detailed in Chapter 6.

As explained in Section 3.2.2, the semi-circular coil heads are split in two to reduce the peak magnetic field in the conductor. Between the two sections of the heads, copper "crescent" shaped pieces are inserted. One of the two crescents of 


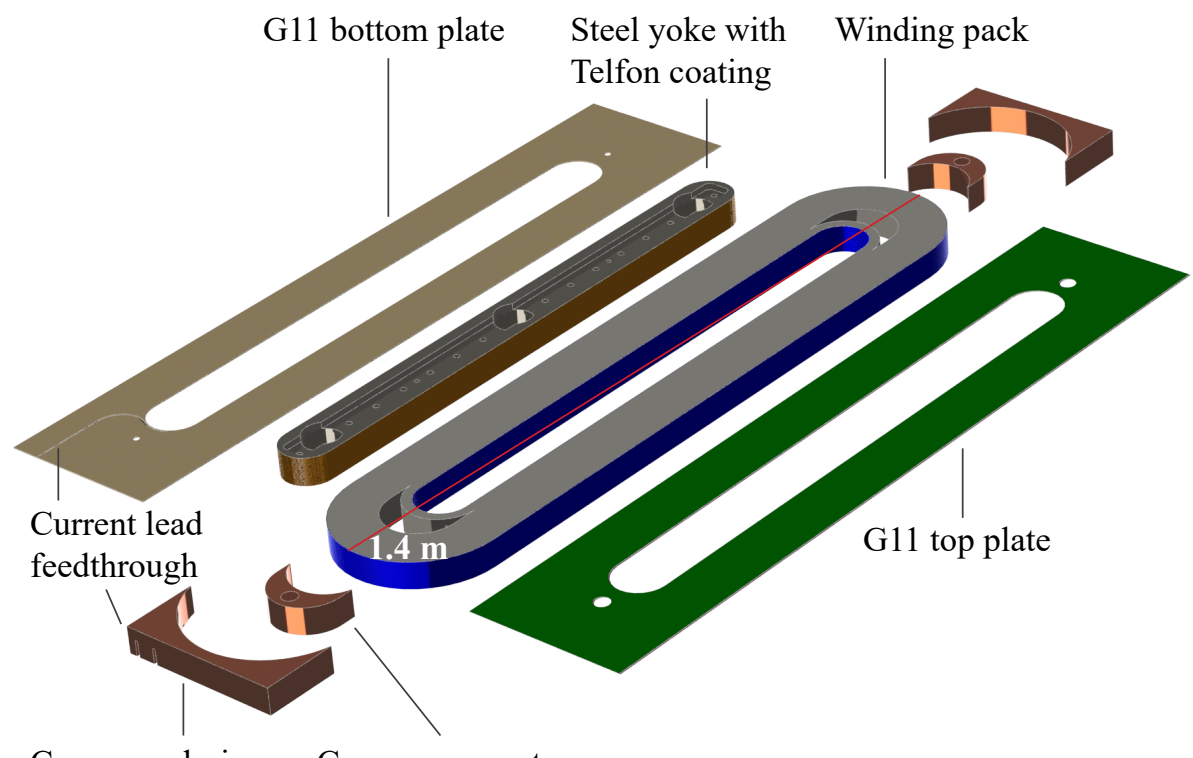

Copper end-piece Copper crescent

Figure 3.11: Exploded view of the major components in each of the three coils.

each coil incorporates a temperature sensor. Copper is chosen for its high thermal conductivity and because its thermal contraction is close to that of the winding pack (discussed in Chapter 5). Since a rectangular coil is easier to shim into place inside the mechanical enclosure (Chapter 6), copper end-pieces are also put on the ends of the winding pack heads to obtain a rectangular shape. On one side of the winding pack, a $2 \mathrm{~mm}$ thick G11 plate $^{3}$ includes a slot that allows to pass the current lead. The current lead consists of two superconducting wires soldered together over the entire length. On the other side of the winding pack, a $0.5 \mathrm{~mm}$ thick G11 plate is glued after winding.

The main constituent of the winding pack is the superconducting wire, manufactured by Bruker EAS GmbH, see Table 3.2. The diameter of $1.44 \mathrm{~mm}$, is a compromise between the number of turns in the coils (determining the selfinductance of the magnet and the ease of winding), and the operating current of $300 \mathrm{~A}$. The operating current is limited by the heat load on the cryocooler from the current leads. Such thermal considerations are further discussed in Chapter 5.

On the other hand, a too high self-inductance is also not preferred, since it

\footnotetext{
${ }^{3} \mathrm{G} 11$ is a class of laminated composites consisting of epoxy and glass fabric, commonly used in cryogenic environments due to its high mechanical strength [38]. It has a low thermal conductivity and high electrical resistivity.
} 
Table 3.2: Properties of NbTi/Cu superconductor used in the MDS demonstrator magnet.

\begin{tabular}{|c|c|}
\hline Property & Value \\
\hline Insulated diameter & $1.444 \mathrm{~mm}$ \\
\hline Bare diameter & $1.362 \mathrm{~mm}$ \\
\hline Twist pitch & $92.5 \mathrm{~mm}$ \\
\hline $\mathrm{RRR}^{\mathrm{a}}$ & 111 \\
\hline$I_{\mathrm{c}}$ at $5 \mathrm{~T}$ background field and $4.2 \mathrm{~K}$ & $1921 \mathrm{~A}$ \\
\hline$n$-value at $5 \mathrm{~T}$ & 59 \\
\hline Insulation material & Formvar ${ }^{b}$ \\
\hline Copper-to-non-copper ratio & $1.35: 1$ \\
\hline Number of NbTi filaments & 132 \\
\hline Filament diameter & $75 \mu \mathrm{m}$ \\
\hline Length & $6.3 \mathrm{~km}$ \\
\hline \multicolumn{2}{|c|}{$\begin{array}{l}\text { a } R R R \text { or residual resistance ratio is the ratio between } \\
\text { the electrical resistance at room temperature ( } 0 \text { or } \\
20{ }^{\circ} \mathrm{C} \text { ) and at cryogenic temperature [42]. Usually } \\
4.2 \mathrm{~K} \text { is taken as the low-side temperature for non- } \\
\text { superconducting materials. The RRR-value can be } \\
\text { used as an indication of the purity of a metal. As the } \\
\text { electrical- and thermal resistance of metals are linked, } \\
\text { the RRR allows an estimate of the thermal conductiv- } \\
\text { ity of a metal at cryogenic temperatures. } \\
{ }^{b} \text { Formvar is a trademark name for polyvinyl formal. } \\
\text { It has a thermal rating of } 105^{\circ} \mathrm{C}[42] \text {. }\end{array}$} \\
\hline
\end{tabular}

causes an increase of voltage across the coils when the magnet is ramped up or down, for example in a fault scenario.

This voltage can not be too high, in order to prevent electric arcs that could destroy the coil or the connected circuit. In between vacuum and atmospheric pressure, the electrical field required to start an arc is lower than at a high vacuum or at atmospheric conditions [43]. Such 'dangerous' pressure level can potentially occur due to a leak in the cryostat, and thus overly high voltages need to be avoided in the system. The expected voltages in the demonstrator coils are detailed in Section 5.3. 
The selected NbTi wire can handle a transport current of $300 \mathrm{~A}$ at $5.2 \mathrm{~T}$ and $4.5 \mathrm{~K}$ with a $2 \mathrm{~K}$ temperature margin. The concept of temperature margin is further discussed in Section 3.2.3. The system requires a single pair of current leads, since the coils are placed in a series network. The cryogenic load of the system is detailed in Chapter 5 .

\subsubsection{Summary}

For MDS, a superconducting electromagnet can be used to generate the required gradient in the magnitude of the magnetic field. This gradient ideally only has a component perpendicular to the plane of the coil system. The main question addressed in this section is which practical coil configuration optimally generates such a required magnetic field profile. Magnet layouts were introduced with two possible types of racetrack coils, 'wide' and 'narrow', and configurations with different number of coils were compared. The wider coils are unsuitable due to the presence of zero-field nodes in the separation area.

When selecting the optimum number of coils for an MDS system, one needs to distinguish the two different strategies for driving feed particles through the fluid bed. The method selected here is to tilt the magnet with respect to the horizontal plane and thus to create a horizontal component of the magnetic force.

The vertical distance between the magnet and "problem areas" in which this horizontal component has the wrong direction (i.e. pushing the particles back) depends on the number of coils and on the tilt angle. This vertical distance determines the maximal usable fluid bed depth and hence the separation performance of an MDS system. For a first NbTi demonstrator designed for the separation of electronic material, an angle of $12^{\circ}$ is selected. A configuration consisting of three flat racetrack coils provides the largest useable fluid bed depth not just for this angle, but for a wide range of tilt angles. The fluid bed volume available for separation is comparable for three- and five coil systems at low angles, whereas at large angles the five-coil system provides a larger effective fluid bed volume. A four-coil configuration performs worse at all tilt angles.

The comparison between coil layouts is based on a $2 \mathrm{D}$ analysis of the magnetic field profile, but the selected final layout was verified with a more detailed $3 \mathrm{D}$ analysis taking into account the ends of the three racetrack coils. 


\subsection{Estimate of potential performance of superconducting MDS}

$\mathrm{T}^{\mathrm{N}}$ this section the MDS performance is estimated that can be expected when 1 using superconducting magnets. As a figure of merit to quantify this 'performance', the magnitude of the average vertical magnetic field gradient $\left|\nabla_{z} H\right|$ at the bottom of the fluid bed is used. This straightforwardly allows to compare different layout designs, as well as different superconducting materials, but of course it makes abstraction of issues such as ease of production, thermal- and mechanical management, protection, etc... These issues are discussed elsewhere in the dissertation.

As explained in Chapter 2, a stronger field gradient $\left|\nabla_{z} H\right|$ leads to a higher magnetic buoyancy force $\mu_{0} M_{\mathrm{s}} V\left|\nabla_{z} H\right|$. For feed streams that include relatively heavy materials, such as electronic material, this is an essential prerequisite. However also a second, more general, benefit of high field gradients is that they allow the use of a more diluted ferrofluid (a lower $M_{\mathrm{s}}$ value) while maintaining the same separation performance. Such dilution lowers operational cost, to an extent that depends of the density range involved, i.e. on the required lifting force. The benefits need to be balanced against the cost of the magnet, since increasing the gradient generally involves the generation of a stronger magnetic field, which can be achieved by using a higher-performance superconductor or simply by using more conductor, both resulting in more ampere-turns.

The translation from conductor volume to conductor costs in principle allows to make such an explicit comparison between capital cost and operational expenditure (dominated by the replenishing of lost ferrofluid). However, such an exercise is not presented in this dissertation, since it is quite case-specific. E.g. the most economical superconductor for the separation of electronic material is likely different from that for the separation of light plastics. The same goes for the optimum operating temperature. Furthermore, since both ferrofluid- and superconductor cost are relevant to such an analysis and since both are expected to change with time, such estimates are left to future design teams.

Nevertheless, one can ask: "How strong is the vertical magnetic field gradient that can be created in the fluid bed?", "How does this value depend on the amount of conductor material" or "How do the answers to these questions change for a different superconducting material?" In this section a systematic approach to these questions is worked out by first analysing the potential performance of the demonstrator NbTi magnet as a function of its winding pack thickness, in other words the obtainable maximum $\mu_{0}\left|\nabla_{z} H\right|$ value that can be achieved within a given materials budget. 
This approach is then followed by considering technical superconductors. The main goal of this exercise is to gain a sense of what is possible with different materials, for which we limit the design space to just a few cases ${ }^{4}$. Some of the estimates are likely to be based on an overly optimistic mechanical design. These can be viewed as yielding an upper bound of the achievable vertical magnetic field gradient. The performance estimates can then be used as a starting point for detailed designs of future superconducting MDS magnets.

$\mathrm{NbTi}$ has been selected as the superconductor for the superconducting demonstrator at the start of the research project. This choice was motivated by NbTi's high maturity as a technical superconductor, wide availability, ease of handling and low conductor cost for low- to medium magnetic field applications compared to other superconductors [38].

The evaluation starts by assuming an application that requires an MDS magnet based on three racetrack coils with a width of $300 \mathrm{~mm}$ each. This corresponds to a three narrow-coil system with a periodicity $\lambda$ of $600 \mathrm{~mm}$, as derived in Section 3.1.2.3. With the number, type, and width of the coils fixed, the winding pack thickness $d_{\text {coil }}$ is the only geometrical parameter in the cross-sectional profile of the winding pack that can be varied. Thus the conductor volume scales linearly with the winding pack thickness. The bottom of the fluid bed is placed parallel to the top surface of the coils, at a distance $z_{0}$.

The first question to be answered then becomes: "What is the obtainable average magnitude of the vertical magnetic field gradient at this height, $\mu_{0} \mid \nabla_{z} H(z=$ $\left.z_{0}, d_{\text {coil }}\right) \mid$, as a function of the coil thickness?"

Two approaches to this question are followed. In the first, the demonstrator's mechanical design is extrapolated. It is based on a symmetric mechanical enclosure, i.e. under the assumption that the support structures on top of- and below the coils have the same thickness. Increasing the number of ampere-turns in the magnet increases the Lorentz forces generated by the coils, and this in turn places an increased demand on the required strength of the mechanical enclosure. This can lead to an increased coil-to-fluid distance, and thus to a smaller performance increase than initially expected.

The second, more optimistic, approach assumes that a mechanical design can be found for which the distance between coils and ferrofluid need not be increased when the magnet is made more powerful, for example by using the space on the bottom of the coils to handle the force. This will be referred to as an asymmetric approach. Some potential designs in this direction are explored in Section 4.4.7. The symmetric and asymmetric performance estimates can be viewed

\footnotetext{
${ }^{4}$ Specifically, each material will pose different thermal and mechanical boundary conditions and therefore in principle would require a dedicated design. Such detailed material-specific designs for other superconductors fall outside the scope of this thesis.
} 
as lower- and upper bounds, respectively. Also included is the performance of double-walled bath-cooled designs, to indicate the significant improvements that conduction-cooled MDS systems bring.

A vertical gradient of the magnetic field magnitude of magnitude $20 \mathrm{~T} / \mathrm{m}$ at the bottom of the fluid bed can be realized with the NbTi demonstrator ${ }^{5}$. It will be argued that this performance is near the maximum that one can obtain from NbTi at a $4.5 \mathrm{~K}$ operating temperature. Thus the symmetrical approach is not a limiting factor for NbTi-based MDS systems. This $4.5 \mathrm{~K}$ was deemed to be an achievable maximum temperature at the start of the design process for this conduction-cooled magnet using a single cryocooler. Chapter 5.2 deals with a detailed estimate of the maximum temperature in the coils.

Using the same shape of mechanical enclosure for the coils as in the demonstrator design (i.e. using the symmetrical approach) allows to estimate the performance for different technical superconductors. An increase of $80 \%$ in vertical magnetic field gradient looks feasible with a $\mathrm{Nb}_{3} \mathrm{Sn}$ - or ReBCO conductor operating at $4.5 \mathrm{~K}$ compared to $\mathrm{NbTi} \mathrm{MgB}_{2}$-based $\mathrm{MDS}$ magnets yield values similar to those found for $\mathrm{NbTi}$ at this operating temperature. ReBCO shows to be promising at higher temperatures as well. Bi-2223 is expected to yield results similar to those of $\mathrm{Nb}_{3} \mathrm{Sn}$ and $\mathrm{ReBCO}$, but no calculations were made to support this. $\mathrm{Bi}-2212$ is not considered due to the required over-pressure heat-treatment [44].

If a mechanical design can be found for which the distance between coils and ferrofluid need not be increased when the magnet is made more powerful (e.g. using the asymmetrical approach), $\mathrm{Nb}_{3} \mathrm{Sn}$ coils may offer a factor three increase in vertical magnetic field gradient compared to the demonstrator, and ReBCO magnets even a factor four.

\subsubsection{Structure of the performance estimate analysis}

In essence, performance estimates for superconducting magnets are made using the "load line" principle $[38,39]$. The linear relation $H_{\text {peak }}=a J$ between the current density $J$ in the electromagnet and the peak magnetic field $H_{\text {peak }}$ on the windings is derived from the geometry of the coil(s) with Ampere's (or BiotSavart's) law. This relation is then compared to the specific critical current density $J_{\mathrm{e}}(H, T)$ of the superconducting material, which in general decreases monotonically with magnetic field $H$ (and temperature $T$ ). The intercept of the two relations therefore gives the maximum current density that the windings can carry before becoming normal. With this current density $J_{\max }$ established, the corresponding magnetic field $H$ can be calculated everywhere.

\footnotetext{
${ }^{5}$ This does not include the small effect on the vertical magnetic field gradient of tilting the system by $12^{\circ}$. The performance estimates obtained in this section thus correspond to an un-angled system.
} 
In our case, there are two main refinements that need to be considered. As the winding pack thickness $d_{\text {coil }}$ increases, the load line expression changes, i.e. the proportionality constant $a$ between $H_{\text {peak }}$ and $J$ is thickness-dependent. Taking into account this dependence is sufficient to estimate the performance for the asymmetric mechanical enclosure.

For the symmetrical mechanical design of the cold mass, a second complication is present, involving mechanical stress due to Lorentz forces and the structural reinforcement required to protect the coils. As the current and magnetic field increase, so do the forces (in principle quadratic with current) and the reinforcement structure needs to become bulkier, which typically increases the distance between the coils and the ferrofluid.

To take all these effects into account (coil geometry, superconducting material choice and mechanical structure), a systematic approach is needed.

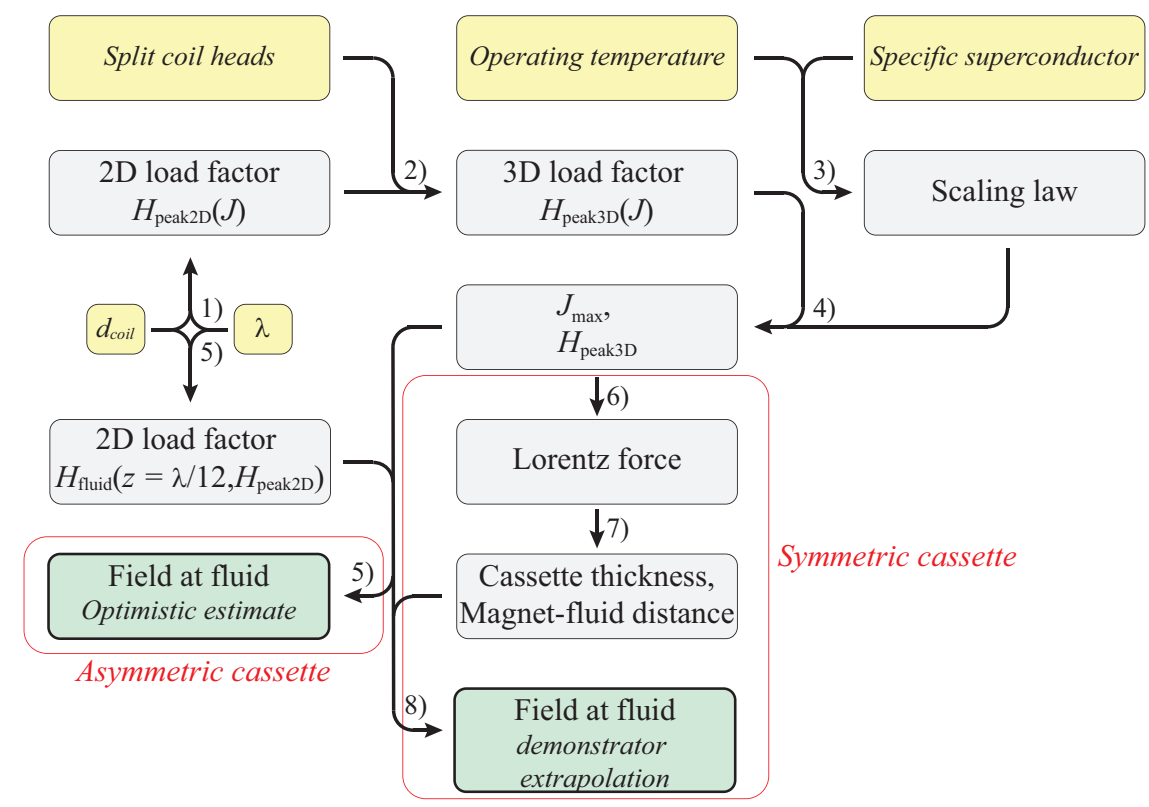

Figure 3.12: Overview of steps to estimate the average magnetic field magnitude at the ferrofluid bed. Inputs that the designer can vary in the algorithm are the thickness of the winding pack $d_{\mathrm{coil}}$, the characteristic size $\lambda$, the amount of shaping of the end sections, the type of conductor and the operating temperature.

For calculating and optimizing the magnetic field gradient, the steps 1 to 8 , illustrated in Figure 3.12, are followed:

1. The relation between the peak magnetic field in the winding pack and the 
current density as a function of the relative coil thickness $d_{\text {coil }} / \lambda$ is taken as a starting point. This relation has been derived in Section 2.4 for a $2 \mathrm{D}$ cross-section using Fourier analysis;

2. Since the peak magnetic field in a racetrack coil is located in the curved head sections, it is this location that limits the performance of the magnet. The shape of these sections is thus optimized to minimize the ratio of peak magnetic field in the curved sections relative to the one in the center of the straight sections;

3. Using scaling laws specific to the superconducting material one can relate the critical current density to the magnetic field and the operating temperature. The scaling laws for state-of-the-art materials are presented in Appendix A;

4. This allows to estimate the maximum current density in the coils as a function of the winding pack thickness, as well as of the maximum magnetic field in the conductor;

5. Since the relation between peak magnetic field in the conductor and the average magnetic field at a fixed distance from the coils is known (Section 2.4) the peak magnetic field found in 4) also allows to estimate the achievable magnetic field in the fluid bed.

If one would disregard mechanical considerations entirely, the analysis would finish here. However, as explained above, a higher magnetic field implies a higher force and stress and hence more robust support structures. This will generally require more distance between the coils and the ferrofluid.

To estimate the coil-to-fluid distance, some extra steps are needed. These are based on an extrapolation of the NbTi demonstrator mechanical design;

6. With the magnetic field magnitude on the conductor, the current density and the geometry determined in step 4, one can calculate the Lorentz force, which determines the required strength of the mechanical enclosure of the coils;

7. The Lorentz force thus fixes the required thickness of the mechanical cassette for a given structural material used;

8. This thickness in turn is used to estimate the coil-to-ferrofluid distance, and therefore the achievable magnetic field gradient;

9. Also of interest is the case in which the cassette thickness does not need to increase with larger Lorentz forces. Performance estimates of the magnetic 
field at the fluid bed for this "optimistic" mechanical design scenario are also included.

\subsubsection{Minimizing the peak magnetic field in head sections (step 2)}

A simple racetrack coil has its magnetic peak field located in turns at the inner surface of the semi-circular heads. This magnetic field is $19 \%$ higher than the maximum magnetic field in the $y$-symmetry plane when using the narrow coil geometry, discussed in Section 3.1.2, and with a relative winding pack thickness of $\lambda / 12$. As the current density in the coils is considered to be uniform, it is this peak magnetic field that limits the potential performance of the magnet since it restricts the maximum current density one can use for a given operating temperature and given type of superconductor. Minimizing this peak field for a given current density is thus beneficial.

By subdividing the curved ends of the racetrack coil in multiple sections, the peak magnetic field can be reduced. Here only a division of the semi-circular sections into two parts is considered, as illustrated in Figure 3.13.

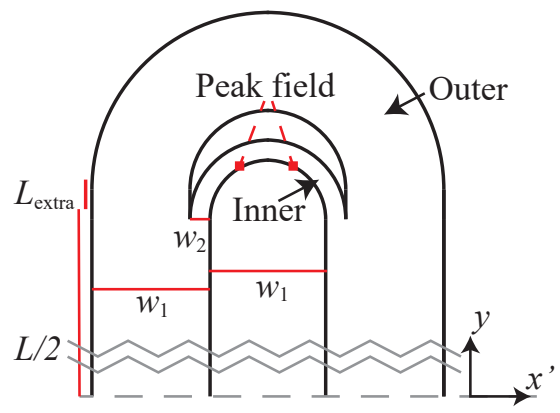

Figure 3.13: Schematic of split heads. The heads are split into two parts. For the demonstrator the inner part has a cross-sectional area of $17 \%$ of the total cross-sectional area of the coil, i.e. $w_{2} / w_{1}=0.17$. $L_{\text {extra }}$ is $0.05 \mathrm{~m}$.

The magnitude of the reduction depends on the extra length $L_{\text {extra }}$ one chooses in the $y$-direction. This is the extra length of the straight sections connecting the outer section of the heads. For a given value of $L_{\text {extra }}$ the ratio of the number of turns in the inner- and outer end-sections can be optimized using FEM calculations.

Figure 3.14 shows the ratio of the peak magnetic field in the semi-circular section relative to that of the straight section (evaluated at the $y$-symmetry plane), as a function of the ratio $w_{2} / w_{1}$. For the demonstrator magnet the inner section has a width of $17 \mathrm{~mm}$ and thus $w_{2} / w_{1}=0.17$. Together with the choice of setting the extra length $L_{\text {extra }}$ to $50 \mathrm{~mm}$ this means that the peak magnetic field in the heads is only $107 \%$ of the peak magnetic field in the straight sections, instead of the value $119 \%$ found without subdivision. 


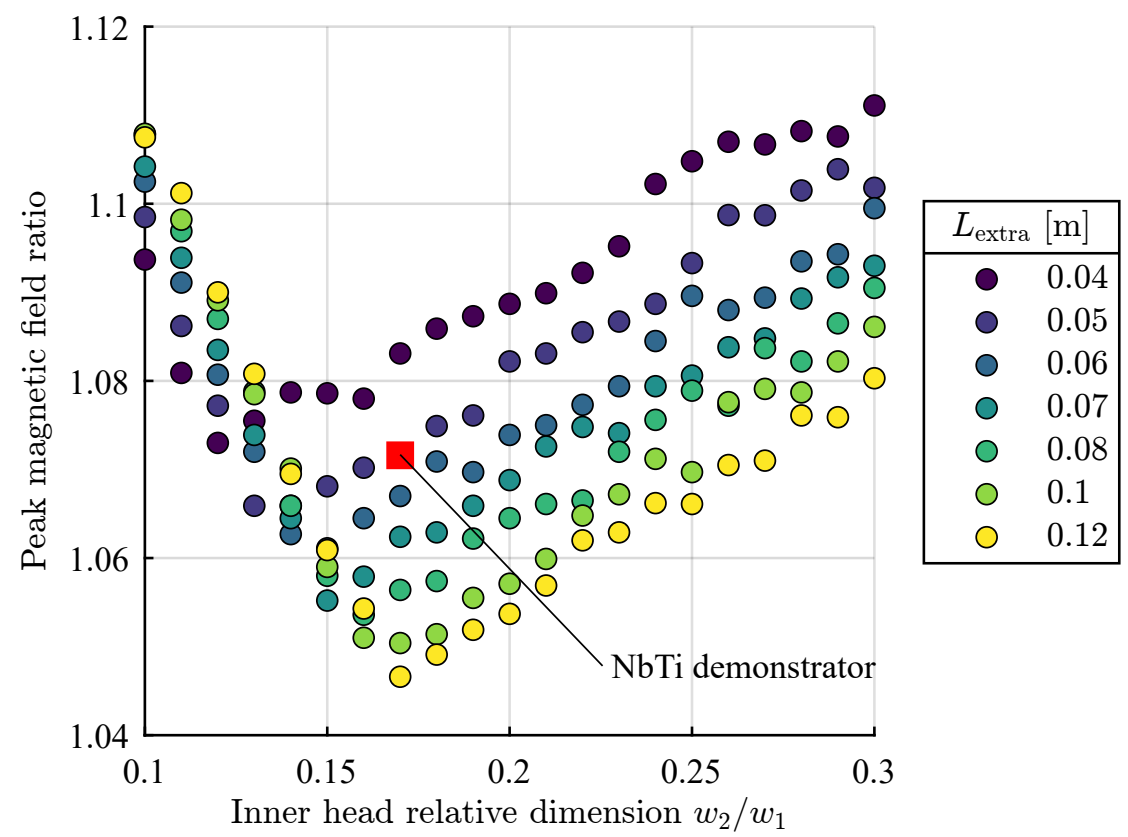

Figure 3.14: Ratio of the peak magnetic field in the winding pack relative to the peak magnetic field at the $y=0$ symmetry plane, as a function of the relative number of turns of the inner coil head section $w_{2} / w_{1}$, as defined in Figure 3.13. The data are generated by COMSOL $3 D$ simulations with boundary conditions reflecting an infinite array of coils. The winding pack thickness was set to $50 \mathrm{~mm}$, the width of both the racetrack coils' legs and inner spacing to $100 \mathrm{~mm}$ and the length of the straight section of the legs, indicated by L in Figure 3.13, to $1 \mathrm{~m}$. Only a single coil is shown. The "infinite array of coils" assumption allows for a faster computation time compared to modelling a finite number of coils, but does not have a large effect on the accuracy of the peak field ratio $(<0.5 \%$ for three coils and up, checked using a fine mesh). Some scatter can be observed in the data-points, due to the difficulty of calculating the maxima accurately while using a relatively coarse mesh. The accuracy of the data is sufficient however to observe trends.

\subsubsection{Temperature margin under operating conditions (step 3)}

The obtainable magnetic field gradient in the fluid bed of course obviously scales with the current density in the coils. The current density $J$ is defined as the current in a single conductor (as supplied by the power supply), multiplied by the number of turns in the coil and divided by the cross-sectional area of one leg of 
the racetrack, in this case $d_{\text {coil }} \times \lambda / 6$. The maximum current density that can safely ${ }^{6}$ be used in the coils is limited and is influenced by the magnetic field in the conductor and by the operating temperature. These three variables span what is called the critical surface of the superconductor [39] ${ }^{7}$. Such a material-specific surface marks the division between the superconducting and normal state. For more information the reader is referred to Appendix A. Assuming a uniform operating temperature $T_{\mathrm{op}}$ of the winding pack, as justified in Chapter 5, it is the peak magnetic field in the conductor that limits the maximum current density.

To ensure safe operation of the magnet, one needs to provide a temperature margin $\Delta T$, defined as the difference between the operating temperature and the current sharing temperature $T_{\mathrm{cs}}{ }^{8}$, and stay below a certain fraction $J / J_{\mathrm{e}}\left(H, T_{\mathrm{op}}\right)$ of the whole-wire critical current density $J_{\mathrm{e}}$. A packing factor $p$ needs to be included to account for the fact that the conductor only fills part of the winding pack volume. Here $p$ is set to 0.67 , implying that $33 \%$ of the winding pack crosssection consists of wire insulation and epoxy and $67 \%$ of the bare conductor. During the winding of the demonstrator coils this turned out to be an accurate estimate. The winding process is detailed further in Chapter 6.

In the analysis, the desired temperature margin $\Delta T$ for $\mathrm{NbTi}$ and $\mathrm{Nb}_{3} \mathrm{Sn}$ is set to $2 \mathrm{~K}$. This value is conservative when operating around $4 \mathrm{~K}$ and based on practical experience with low-temperature superconducting magnets [45]. For the other conductors, the current density $J / p$ is placed at $80 \%$ of the whole-wire engineering critical current density $J_{\mathrm{e}}$, which for these conductors is a more restrictive requirement than the $2 \mathrm{~K}$ margin. This is because their $J_{\mathrm{e}}$ drops off less steeply with increasing temperature. The scaling laws $J_{\mathrm{e}}(H, T)$ that were used for the different materials are presented in Appendix A. In this chapter the operating temperature $T_{\mathrm{op}}$ of the coils is set to $4.5 \mathrm{~K}$ unless specified otherwise.

The following values of the whole-wire critical current density $J_{\mathrm{e}}$ were used:

- $\mathrm{NbTi}$ and $\mathrm{Nb}_{3} \mathrm{Sn}$ at $6.5 \mathrm{~K}$;

- $\mathrm{MgB}_{2}$ at $4.5 \mathrm{~K}$, multiplied by 0.8 ;

- ReBCO multiplied by 0.8 , at $4.5 \mathrm{~K}, 30 \mathrm{~K}$ and $50 \mathrm{~K}$. For this anisotropic conductor, the worst-case magnetic field orientation is considered, with the magnetic field perpendicular to the tape plane [46]. We assume that the racetrack coils are made from stacked pancake coils.

\footnotetext{
"Safe" in this context means with limited risk of the superconductor reverting back to the normal state. Thermal stability and quench behaviour are further discussed in Chapter 5 .

${ }^{7}$ The effects of mechanical strain and stress are not included here.

${ }^{8}$ At the current- and magnetic field dependent temperature $T_{\mathrm{cs}}(J, H)$ the superconductor becomes normal. $T_{\mathrm{cs}}(J, H)$ is thus essentially an alternative formulation of the critical surface $J_{\mathrm{e}}(H, T)$.
} 


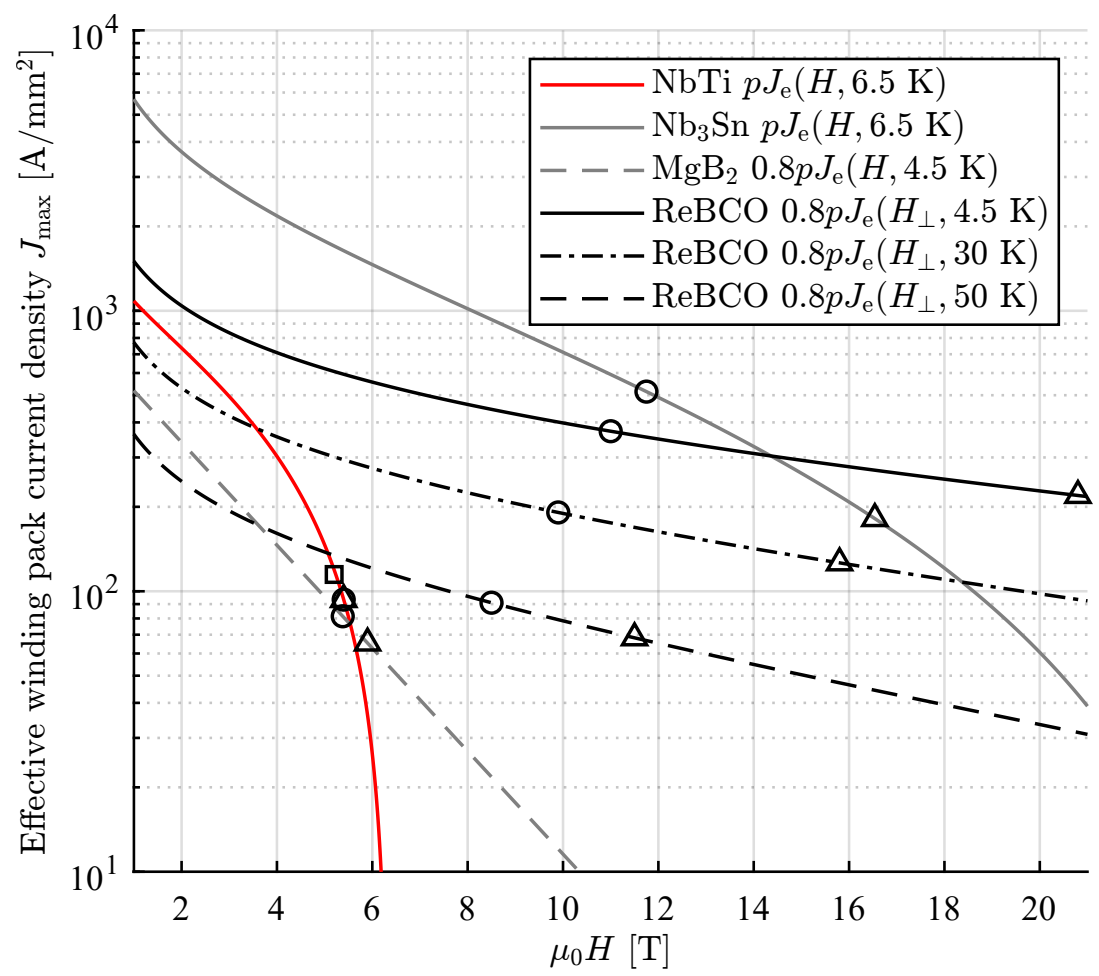

Figure 3.15: Maximum current density averaged over the cross-section of the winding pack, as a function of magnetic field, shown for the different materials considered. To give an idea of various operating points, several possible geometries are presented with markers. Round markers indicate optimized operating points based on an extrapolation of the NbTi demonstrator, see Table 3.1, the square marker the operating point of the demonstrator itself. The slight difference in the two points for NbTi is the result of the conductor performing better than the initial specification. The triangular markers correspond to the obtained values for a $150 \mathrm{~mm}$ thick winding pack.

The current densities, multiplied by the packing factor $p$, are shown in Figure 3.15. With the chosen margins, $\mathrm{Nb}_{3} \mathrm{Sn}$ offers the highest allowed current densities up to a magnetic field of $14 \mathrm{~T}$, where it is overtaken by ReBCO. At low magnetic fields NbTi performs similarly to ReBCO, but its $J_{\mathrm{e}}$ value drops off rapidly with increasing magnetic field. 


\subsubsection{Obtainable current density and magnetic field at conductor (step 4)}

With the safety margins as discussed above, the maximum current density $J_{\max }$ that can be achieved with different materials can be determined for a given winding pack thickness $d_{\text {coil }}$, yielding the maximum number of ampere-turns for that amount of conductor and geometry. This maximum current density can be directly related to the peak magnetic field in the conductor by using the $J_{\max }(H)$ relations shown in Figure 3.15.

To determine for the different considered superconductors the peak magnetic field in the conductor as a function of the thickness of the coils, first the factor $a\left(d_{\text {coil }}\right)$ between the magnetic field and the current density is determined for each specific thickness. The maximum magnetic field magnitude found in a $2 \mathrm{D}$ crosssection at the $y=0$ symmetry plane is multiplied by a factor 1.07 to obtain the peak magnetic field in the split heads, as discussed in Section 3.2.29. This relation between current density and maximum field, $H_{\text {peak }}=a\left(d_{\text {coil }}\right) J$, depends on the load factor $a\left(d_{\text {coil }}\right)$ that is specific to the geometry. This load line is then compared with the scaling law of the respective superconductors. To determine the maximum current density that is allowed, one numerically solves either

$$
J=p J_{\mathrm{e}}\left(H=a\left(d_{\text {coil }}\right) J, T=T_{\mathrm{op}}+\Delta T\right),
$$

for the temperature margin of $\mathrm{Nb}_{3} \mathrm{Sn}$ and $\mathrm{NbTi}$, or

$$
J=0.8 p J_{\mathrm{e}}\left(H=a\left(d_{\text {coil }}\right) J, T=T_{\mathrm{op}}\right),
$$

for the current margin in $\mathrm{ReBCO}$ and $\mathrm{MgB}_{2}$. This algorithm yields an operating point $(H, J)$ placed somewhere on the curves shown in Figure 3.15.

The number of ampere-turns in the winding pack, calculated from the maximum allowed current density and the thickness of the winding pack, can thus be derived from the peak magnetic field $H_{\text {peak }}$ in the winding pack.

In Figure 3.16 is presented how $H_{\text {peak }}$ increases with $d_{\text {coil }}$. Increasing the winding pack thickness has less and less effect on the value of $H_{\text {peak }}$. As $d_{\text {coil }}$ increases, the turns at the top and bottom of the winding pack are further away from its center plane where the peak field is located and thus contribute less and less. Additionally, the increasing peak magnetic field decreases the achievable current density, which further flattens the curves.

\footnotetext{
${ }^{9}$ This factor can be larger or smaller, depending on the amount of subdivision one applies. For some superconductors, one may have to resort to specially shaped heads to minimize $I_{\mathrm{c}}$ degradation due to bending if the minimum bending radius is not large enough for the desired $\lambda$.

Also, the ratio could depend slightly on the relative winding pack thickness $d_{\text {coil }} / \lambda$. This effect is not taken into account.
} 
In the next section the relation between $H_{\text {peak }}$ and the "useful" magnetic field in the fluid bed is made.

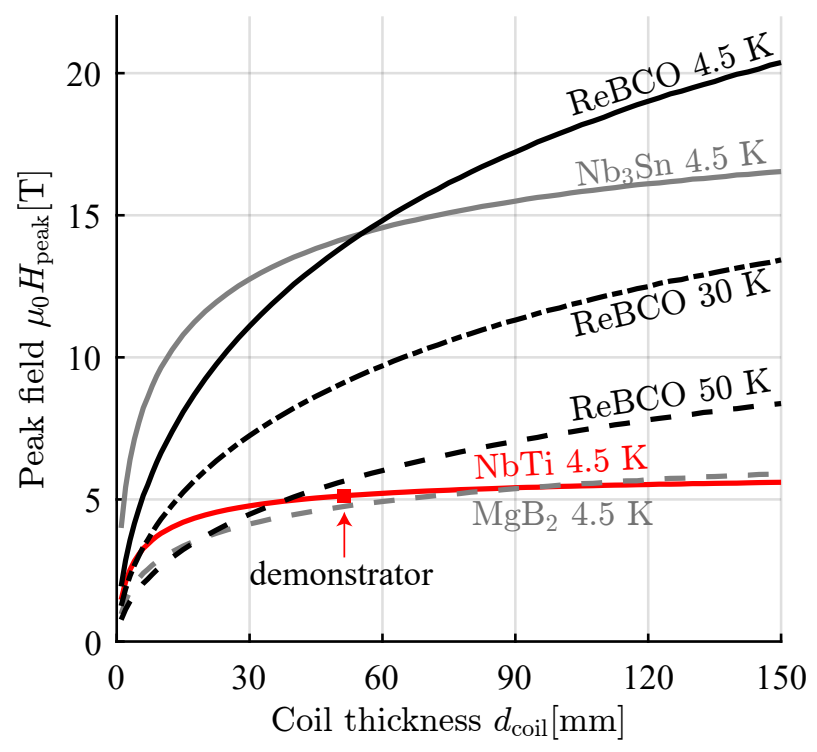

Figure 3.16: Maximum magnetic field in the winding pack for a 3-coils system with periodicity $\lambda=600 \mathrm{~mm}$, as a function of winding pack thickness $d_{\text {coil }}$, for different superconductors. For small coil thicknesses an increase of the thickness has a stronger effect on the peak magnetic field than for thicker coils.

\subsubsection{Performance of the NbTi demonstrator magnet}

With the NbTi demonstrator project the available budget allowed to manufacture three racetrack coils with a winding pack thickness of $50 \mathrm{~mm}$. This results in a maximum magnetic field $\mu_{0} H_{\text {peak }}$ on the conductor of $5.2 \mathrm{~T}$, as shown in Figure 3.16 .

The resulting average vertical magnetic field gradient at the bottom of the field bed was calculated using 3D FEM to be $20 \mathrm{~T} / \mathrm{m}$. The corresponding magnetic field profiles are given in Appendix B. In this evaluation the distance between the top plane of the coils and the ferrofluid, $z_{0}$, is $50 \mathrm{~mm}$. This value includes both the mechanical coil enclosure (Section 4.4), and the vacuum vessel (Section 4.2).

To estimate the potential performance for other superconductors and different winding pack thicknesses, some scaling arguments are used instead of performing full 3D electromagnetic- and mechanical calculations. These scaling arguments are discussed in the next sections. 


\subsubsection{Magnetic field at fluid bed (step 5)}

The ratio between $H_{\text {peak }}$ and the average magnetic field magnitude at the bottom of the ferrofluid is known as a function of winding pack thickness (Section 2.4.2). This ratio and the previously derived value of $H_{\text {peak }}$ would in principle allow to estimate the obtainable vertical magnetic field gradient at the bottom of the fluid bed $z_{0}$ via

$$
\begin{aligned}
\mu_{0} \nabla_{z} H\left(z_{0}, d_{\text {coil }}\right) & =-\frac{2 \pi \mu_{0}}{\lambda} H_{\text {mean }}\left(z_{0}, d_{\text {coil }}\right) \\
& =-\frac{2 \pi \mu_{0}}{\lambda} \frac{H_{\text {mean }}\left(z_{0}, d_{\text {coil }}\right)}{H_{\text {peak }}\left(d_{\text {coil }}\right)} H_{\text {peak }}\left(d_{\text {coil }}\right) .
\end{aligned}
$$

Because of the finite size of the system, edge effects play a role in the magnitude of the average magnetic field $H_{\text {mean }}$ at the fluid bed bottom. $H_{\text {mean }}$ will depend on the range of $x$-coordinates one evaluates it over. In Figure 3.17 the ratio between $H_{\text {mean }}$ and $H_{\text {peak }}$ is shown for a three coils system with $x$ running from the leftmost to right-most edges of the coils, that is, $x=[-0.75 \lambda, 0.75 \lambda]$. To proceed further, one needs to take into account how the coil-fluid distance $z_{0}$ changes for a thicker winding pack, due to the need for a stronger mechanical enclosure. To do this, the relation between Lorentz force and magnetic field needs to be established. This is the focus of the next section.

\subsubsection{Lorentz force and coil thickness (step 6)}

Since a stronger magnetic field or larger winding pack thickness also entails a stronger Lorentz force, the mechanical cassette surrounding the coils needs to be stronger. This results in an increased distance between coils and fluid, i.e. in an increase of $z_{0}$ in Eq. 3.9b, and thus in a smaller enhancement of the gradient than expected. Here we estimate how the Lorentz force scales with the number of ampere-turns of the coils. It will turn out that this increase is faster than a simple quadratic relation with the peak magnetic field in the conductor.

The Lorentz force on the coils pushes them outwards in the horizontal plane and compress them vertically. Figure 3.18 and Table 3.3 illustrate the direction of the horizontal force in several sections of the coils. Due to symmetry, only a quarter of the $x^{\prime} y$-plane needs to be shown. The force in the $x^{\prime}$-direction acting on section III is slightly larger than that on section II due to the higher magnetic field in the center coil. Thus the net force in the $x^{\prime}$-direction on sections II and III is directed outwards. The force pushing section I outwards is nearly four times larger. Thus it seems likely that increasing the number of coils only has a minor effect on the total horizontal Lorentz force in the $x^{\prime}$-direction.

Handling the Lorentz force in the $x^{\prime}$-direction is one of the main challenges of 


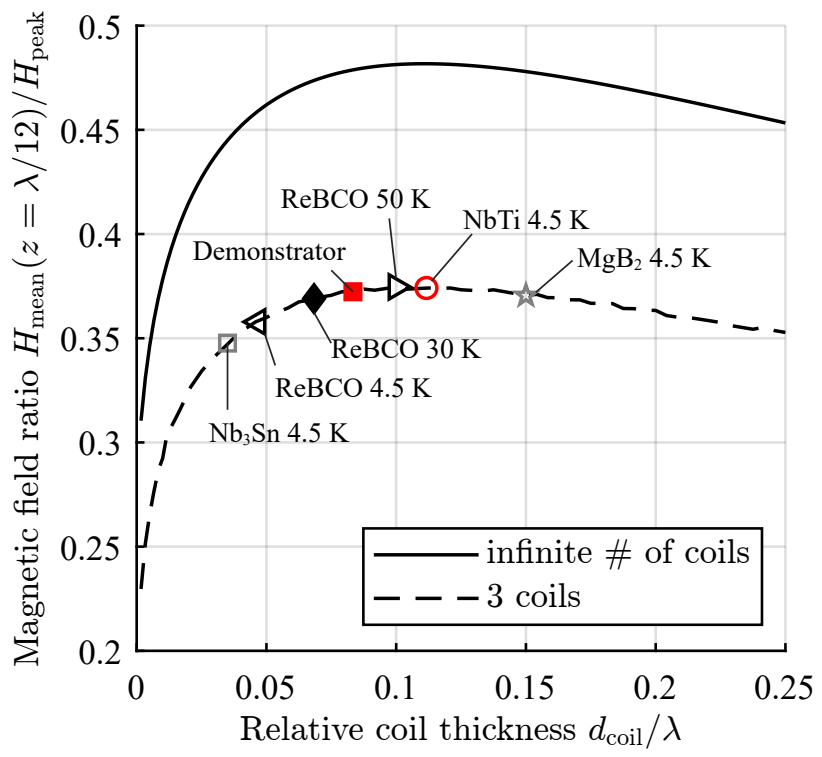

Figure 3.17: Ratio between average magnetic field at the fluid bed and peak magnetic field in winding pack (in $3 D$ ), as a function of the relative winding pack thickness $d_{\text {coil }} / \lambda$. The magnetic field at the fluid bed is evaluated at a relative distance of $\lambda / 12$ above the winding pack. This distance corresponds to the $50 \mathrm{~mm}$ between the coils and the ferrofluid in the NbTi demonstrator design. Ratios for both an infinite array of coils (solid line) and a system of just three coils (dashed line) are shown. For the latter, the magnetic field is evaluated in the range $x=$ $[-0.75 \lambda, 0.75 \lambda]$. Markers indicate the operating points as determined later in this section for designs based on extrapolation of the demonstrator design (Table 3.5).

the mechanical design, as will be discussed in detail in Chapter 4. The net force in the $x^{\prime}$-direction is $68 \%$ higher than in the $y$-direction for this coil layout with a length of the straight sections of one meter. If the straight sections would be shorter than half a meter, the force in the $y$-direction would become dominant. In the remainder of the discussion the straight sections are assumed to be one meter long.

The aluminium alloy cassette surrounding the coils consists of two almost identical machined plates, of 5083-H321 alloy, as detailed in Section 4.4. Topand bottom parts are bolted together. The two halves symmetrically support the horizontal Lorentz force produced by the coils, i.e. each part takes half the total force. During the cool-down, the cassette shrink-fits around the coils thereby 


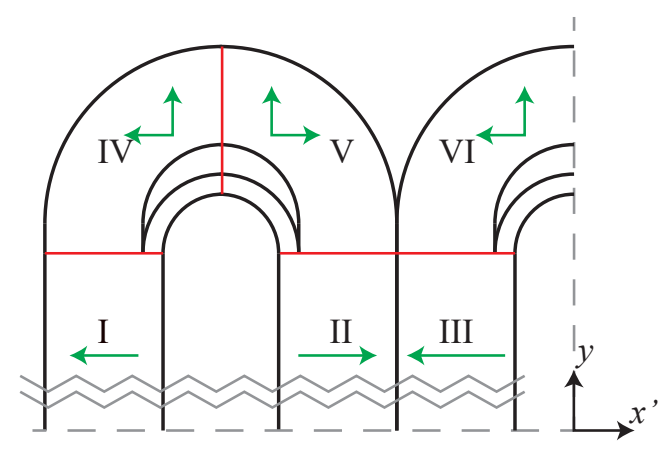

Figure 3.18: Sketch of the in-plane Lorentz forces (green arrows). A quarter of the coils is shown, with the dashed grey lines representing symmetry planes. Section I is pushed outwards, and the net forces in the $x^{\prime}$-direction of section II and III are also negative. Sections IV, V and VI experience net forces in the radial direction. The magnitudes of the forces are given in Table 3.3.

Table 3.3: Lorentz forces in various sections of the NbTi demonstrator coils, at operating current of $300 \mathrm{~A}$. The sections making up one symmetry quadrant are illustrated in Figure 3.18. The forces are calculated with 3D FEM by integrating the local force density $\boldsymbol{J} \times \boldsymbol{B}$ over the volume of the respective sections. For the vertical $z^{\prime}$ force, only the top halves of the coils are considered in the integration.

\begin{tabular}{lrrr} 
Section & $F_{x^{\prime}}[\mathrm{kN}]$ & $F_{y}[\mathrm{kN}]$ & $F_{z^{\prime}}[\mathrm{kN}]$ \\
\hline I & -296 & 0 & -212 \\
II & +576 & 0 & -255 \\
III & -653 & 0 & -255 \\
IV & -101 & +85 & -77 \\
V & +150 & +97 & -84 \\
VI & -165 & +108 & -84
\end{tabular}

providing a pre-compression that counteracts the tensile stress due to the Lorentz force.

When the coils generate a larger Lorentz force, the cassette needs to provide a larger compressive force and thus has to be made thicker, which results in an increased distance between coils and ferrofluid. The limitations imposed by this symmetrical approach for high-gradient MDS magnets are thus of interest. To 
get an estimate of the limitations, the effects of both increasing $d_{\text {coil }}$ and of using different superconducting materials on the required cassette thickness are found.

To determine the required cassette thickness (Section 3.2.8), first the relation between the horizontal Lorentz force and $H_{\text {peak }}$ needs to be known. On first sight one would expect this force to scale with the square power of the magnetic field. This is the case if $d_{\text {coil }}$ is kept constant and the current density is changed. However, in this study not $d_{\text {coil }}$ but the operating margin is kept constant, so increasing $H_{\text {peak }}$ requires a thicker winding pack (Figure 3.16). The total horizontal Lorentz force thus grows faster than quadratic. As shown in Chapter 4, this force determines the required cassette thickness. The sum of the horizontal forces on coil sections I, II and III as a function of the $H_{\text {peak }}$ value is shown in Figure 3.19, for different superconductors.

Fits of the form

$$
F_{\mathrm{L}, x}=a\left(\mu_{0} H_{\text {peak }}\right)^{b}+c\left(\mu_{0} H_{\text {peak }}\right)^{d},
$$

are also included in Figure 3.19. The values of the coefficients $a, b, c$ and $d$ are presented in Table 3.4. $b$ and $d$ indicate a steeper-than-square growth of the Lorentz force when one uses higher field magnets.

Table 3.4: Values of coefficients of Eq. 3.10 for various superconductors obtained by fitting to the calculated Lorentz forces. Coefficient a has units $\left[\mathrm{NT}^{-b}\right]$, coefficient $c$ has units $\left[\mathrm{NT}^{-d}\right] .95 \%$ confidence bounds are indicated by. \pm

\begin{tabular}{llllll} 
Superconductor & $T_{\mathrm{op}}[\mathrm{K}]$ & $a$ & $b$ & $c$ & $d$ \\
\hline $\mathrm{NbTi}$ & 4.5 & $2618 \pm 341$ & $3.18 \pm 0.10$ & $(1.06 \pm 1.47) \cdot 10^{-5}$ & $14.6 \pm 0.81$ \\
$\mathrm{Nb}_{3} \mathrm{Sn}$ & 4.5 & $1306 \pm 114$ & $3.01 \pm 0.04$ & $(3.72 \pm 2.4) \cdot 10^{-6}$ & $10.1 \pm 0.23$ \\
$\mathrm{MgB}_{2}$ & 4.5 & $5443 \pm 198$ & $2.78 \pm 0.04$ & $1.66 \pm 0.49$ & $7.49 \pm 0.15$ \\
$\mathrm{ReBCO}$ & 4.5 & $3527 \pm 115$ & $2.67 \pm 0.02$ & $0.583 \pm 0.134$ & $5.50 \pm 0.07$ \\
$\mathrm{ReBCO}$ & 30 & $4183 \pm 129$ & $2.67 \pm 0.02$ & $1.30 \pm 0.30$ & $5.56 \pm 0.08$ \\
$\mathrm{ReBCO}$ & 50 & $5009 \pm 128$ & $2.69 \pm 0.02$ & $2.93 \pm 0.64$ & $5.68 \pm 0.04$
\end{tabular}




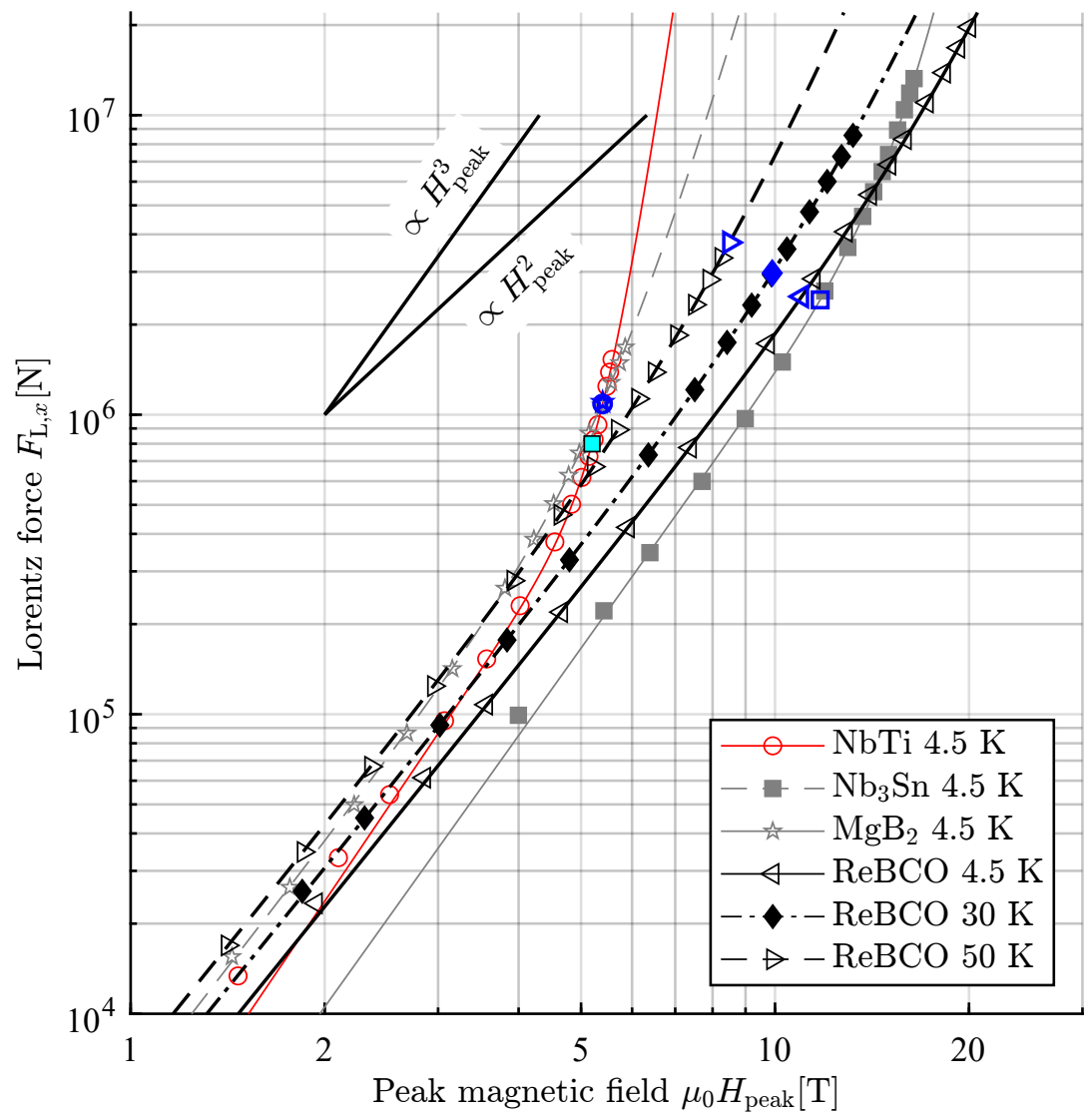

Figure 3.19: Variation of the horizontal Lorentz force on the straight legs of the racetrack coils with changing peak magnetic field and for various superconductors. Markers indicate data calculated with COMSOL, lines indicate fits of the form of Eq. 3.10. Not all simulation data used for the fit are shown, to reduce clutter. The model assumes a 3-coils system with a leg length of $1 \mathrm{~m}$ and characteristic period $\lambda$ of $600 \mathrm{~mm}$. Blue markers indicate the extrapolations based on the MDS demonstrator, see also Figure 3.15, and the cyan marker the demonstrator itself. 


\subsubsection{Required cassette thickness (step 7)}

It is shown in Chapter 4 that the main factor determining the cassette thickness $d_{\text {cas }}$ is the thermal compression that needs to be applied on the winding packs during cool-down, relative to the Lorentz force. Choosing a material with a higher yield strength does not help in keeping the thickness small. The tensile stress in the cassette itself and in the winding pack are of secondary importance, since it is the relative thermal contraction which is the main concern.

Here the required pre-stress is assumed to be such that the transverse stress in the winding packs stays negative after energizing of the coils. For ReBCO-based coils, this may be an overly restrictive demand.

The pre-compression from the shrink-fit of the aluminium alloy cassette around the winding pack during cool-down is calculated for the NbTi demonstrator design (Chapter 4). For non-NbTi systems this compression may be different, due to differences in thermal contraction or stiffness.

Assuming the cassette to be approximately symmetric, i.e. with top and bottom plates of the same thickness, as in the demonstrator magnet design (Section 4.4), a larger Lorentz force implies that the distance between the coils and the ferrofluid needs to increase. The relation between the plate thickness $d_{\text {cas }}$ as a function of the applied Lorentz force is shown in Figure 3.20. $d_{\text {cas }}$ increases quickly with the applied force. The demonstrator's cassette is slightly thicker $(12 \mathrm{~mm}$ instead of $9 \mathrm{~mm}$ ) than needed, in order to obtain a significant mechanical safety margin.

\subsubsection{Performance - demonstrator extrapolation (step 8)}

With an estimate of $d_{\text {cas }}$ as a function of the generated Lorentz forces, the effect on a changing distance between fluid and coils compared to the NbTi demonstrator can be accounted for by multiplying Eq. $3.9 \mathrm{~b}$ with a factor

$$
\exp \left[\frac{2 \pi}{\lambda}\left(d_{\text {cas }}-0.012 \mathrm{~m}\right)\right] \text {. }
$$

This results in the following relation between the vertical magnetic field gradient at the bottom of the fluid bed $z_{0}$ and the thickness of the winding pack $d_{\text {coil }}$ :

$$
\begin{aligned}
\mu_{0} \nabla_{z} H\left(z=z_{0}, d_{\text {coil }}\right)= & -\frac{2 \pi \mu_{0}}{\lambda} \frac{H\left(z=50 \mathrm{~mm}, d_{\text {coil }}\right)}{H_{\text {peak }}\left(d_{\text {coil }}\right)} H_{\text {peak }}\left(d_{\text {coil }}\right) \\
& \times \exp \left[\frac{2 \pi}{\lambda}\left[d_{\text {cas }}\left(F_{\mathrm{L}, x}\left(d_{\text {coil }}\right)\right)-0.012 \mathrm{~m}\right]\right],
\end{aligned}
$$




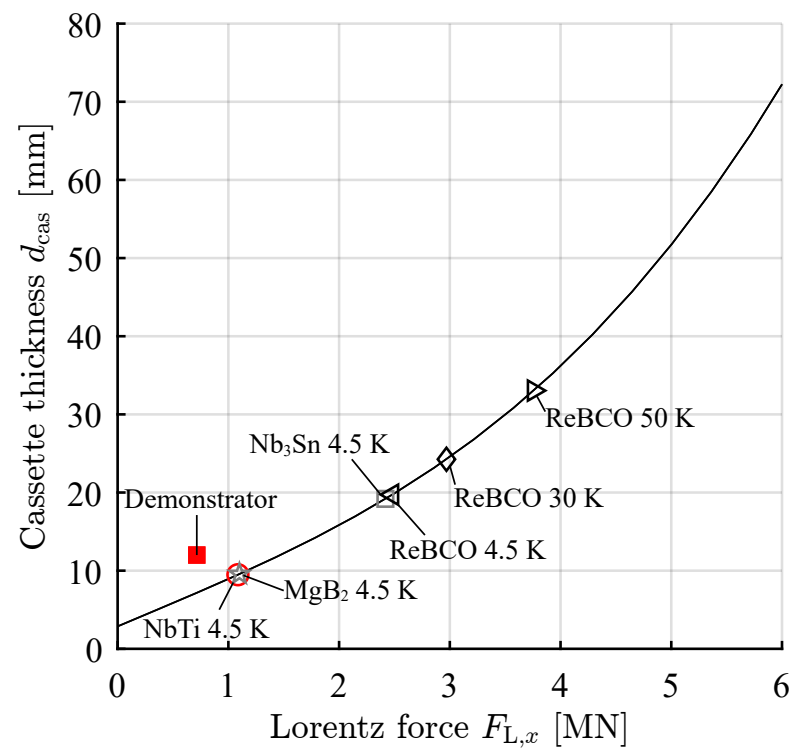

Figure 3.20: Cassette thickness $d_{\text {cas }}$ required to apply sufficient pre-compression to compensate a Lorentz force $F_{\mathrm{L}, x}$. The calculations are made assuming a design in which the top-and bottom cassette parts have the same thickness $d_{\text {cas. }}$.

where $H_{\text {peak }}$ is given by the data in Figure $3.16, d_{\text {cas }}$ by Figure 3.20 and $F_{\mathrm{L}, x}$ by Eq. 3.10. The result is shown for different materials in Figure 3.21 as a function of the winding pack thickness $d_{\text {coil }} .\left|\nabla_{z} H\right|$ first rises quickly with increasing $d_{\text {coil }}$, reaches a maximum and then decreases. The decrease is due to a fast increase of the $d_{\text {cas }}$ required to compensate for the faster increase in $F_{\mathrm{L}, x}$ (Figure 3.19). Shown as well in grey lines is the expected performance of asymmetric designs (i.e. designs for which the distance between the coils and fluid does not need to increase).

The potential performance of NbTi MDS magnets with a characteristic size $\lambda$ of $600 \mathrm{~mm}$ peaks at around $22 \mathrm{~T} / \mathrm{m}$. Compared to the design performance of the demonstrator $(20 \mathrm{~T} / \mathrm{m})$ only marginal improvements can be expected by increasing the winding pack thickness. The current mechanical design is sufficient to get the near-optimal performance out of $\mathrm{NbTi}$, since the curves of the symmetric and asymmetric designs overlap.

$\mathrm{MgB}_{2}$-based systems behave similar to $\mathrm{NbTi}$ with a slight upward shift of the optimum coil thickness. The highest vertical magnetic field gradient can be obtained with $\mathrm{Nb}_{3} \mathrm{Sn}(21 \mathrm{~mm}$ thick winding pack) or ReBCO $(28 \mathrm{~mm})$. Both are able to generate $35 \mathrm{~T} / \mathrm{m}$ at the fluid bed bottom when operating at $4.5 \mathrm{~K}$. Note 

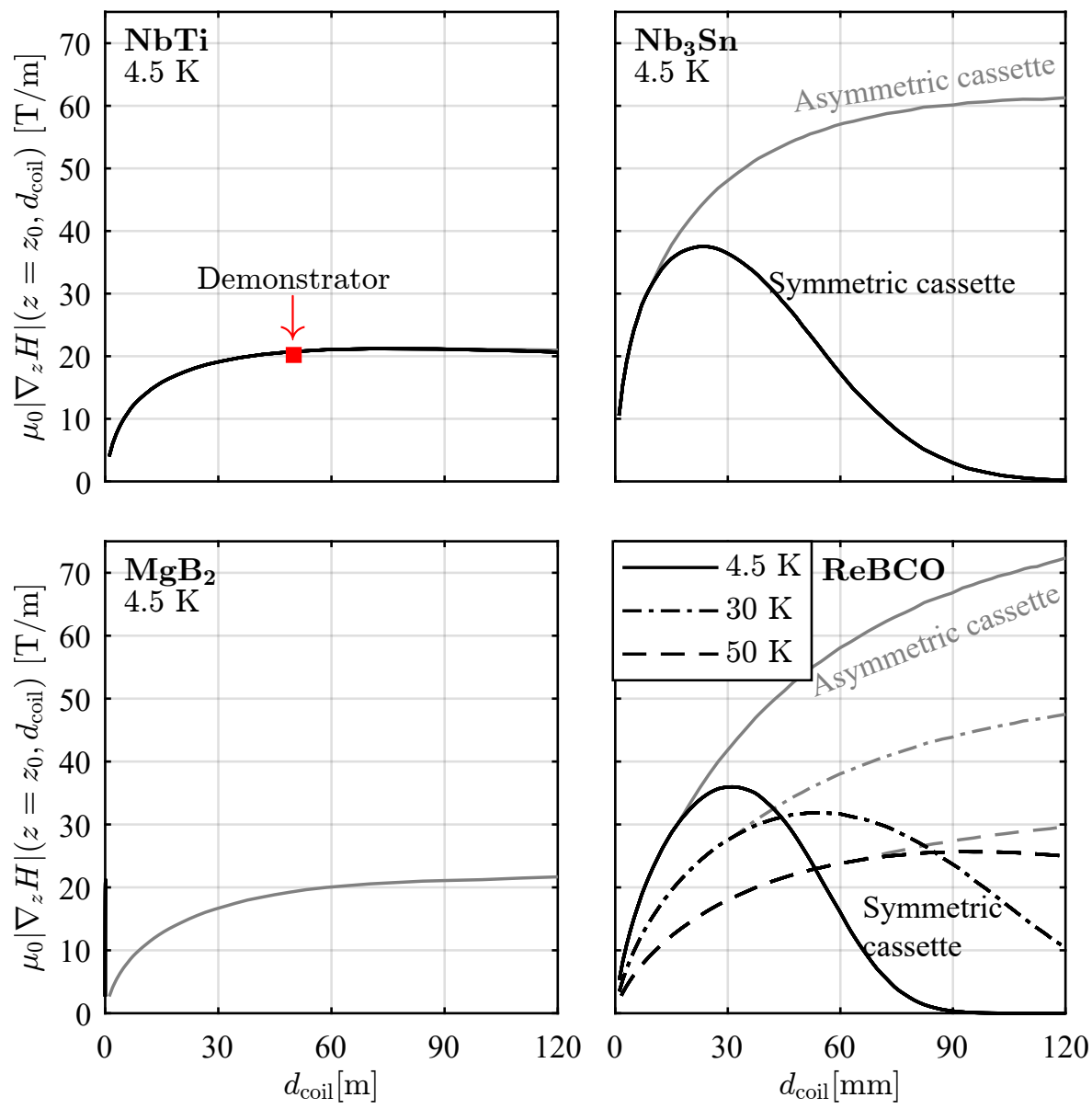

Figure 3.21: Average vertical magnetic field gradient magnitude $\mu_{0}\left|\nabla_{z} H\right|$ at $z_{0}$, the bottom of the fluid bed, as a function of coil thickness $d_{\text {coil }}$. The black lines indicate designs using a symmetrical cassette, grey lines a asymmetrical cassette. For ReBCO, the line style indicates the operating temperature. All calculations assume a 3-coils system with racetrack coil leg length of $1 \mathrm{~m}$ and characteristic period $\lambda$ of $600 \mathrm{~mm}$. The demonstrator design value is indicated with a red rectangle. 
that a scaling law for $\mathrm{ReBCO}$ with the magnetic field perpendicular to the tape is used, whereas the actual peak magnetic field will likely be parallel to the tape. A higher performance may thus be achievable. The cassette thicknesses for the best performing 4.5 K ReBCO- and $\mathrm{Nb}_{3} \mathrm{Sn}$ systems are both around $20 \mathrm{~mm}$.

Increasing the operating temperature of the ReBCO system to $30 \mathrm{~K}$ decreases its potential performance by around $9 \%$, which may be acceptable in view of the advantages of much less stringent thermal requirements. The best performing $30 \mathrm{~K}$ configuration does use more conductor than the best performing $4.5 \mathrm{~K}$ system ( $d_{\text {coil }}$ is 41 vs $28 \mathrm{~mm}$ ).

If a design can be found in which the Lorentz force is handled solely on the bottom of the coils, significant improvements in the $\left|\nabla_{z} H\right|\left(z_{0}\right)$ value can be achieved, see the grey lines in Figure 3.5. Such an asymmetrical mechanical design would result in a performance of $60 \mathrm{~T} / \mathrm{m}$ for $\mathrm{Nb}_{3} \mathrm{Sn}$, and close to $80 \mathrm{~T} / \mathrm{m}$ with $\mathrm{ReBCO}$.

The results of this section are summarized graphically in Figure 3.22. As mentioned before, the optimal superconductor may depend on the specific MDS application, since the cost of the magnet needs to be balanced against the operational cost of replenishing lost ferrofluid.

Table 3.5: Performance estimates of MDS magnets with coils using various technical superconductors. All calculations apply to a 3-coils system with typical size $\lambda$ of $600 \mathrm{~mm}$.

\begin{tabular}{lllllllll} 
Superconductor & \multicolumn{2}{c}{$\begin{array}{c}\text { Field gradient } \\
{[\mathrm{T} / \mathrm{m}]}\end{array}$} & \multicolumn{2}{c}{$\begin{array}{c}\text { Peak field } \\
{[\mathrm{T}]}\end{array}$} & \multicolumn{2}{c}{$\begin{array}{c}d_{\text {coil }} \\
{[\mathrm{mm}]}\end{array}$} & \multicolumn{2}{c}{$\begin{array}{c}d_{\text {cas }} \\
{[\mathrm{mm}]}\end{array}$} \\
\hline & Sym. & Asym. & Sym. & Asym. & Sym. & Asym. & Sym. & Asym. \\
\hline NbTi 4.5 K & 21 & 21 & 5.3 & 5.6 & 67 & 120 & 11 & 11 \\
$\mathrm{Nb}_{3}$ Sn 4.5 K & 36 & 61 & 12 & 16.1 & 21 & 120 & 20 & 11 \\
$\mathrm{MgB}_{2} 4.5 \mathrm{~K}$ & 21 & 22 & 5.4 & 5.7 & 90 & 120 & 12 & 11 \\
$\mathrm{ReBCO} 4.5 \mathrm{~K}$ & 35 & 77 & 11 & 19.3 & 28 & 120 & 20 & 11 \\
$\mathrm{ReBCO} 30 \mathrm{~K}$ & 32 & 58 & 9.9 & 14.7 & 41 & 120 & 24 & 11 \\
ReBCO 50 K & 26 & 43 & 8.5 & 10.8 & 60 & 120 & 33 & 11
\end{tabular}




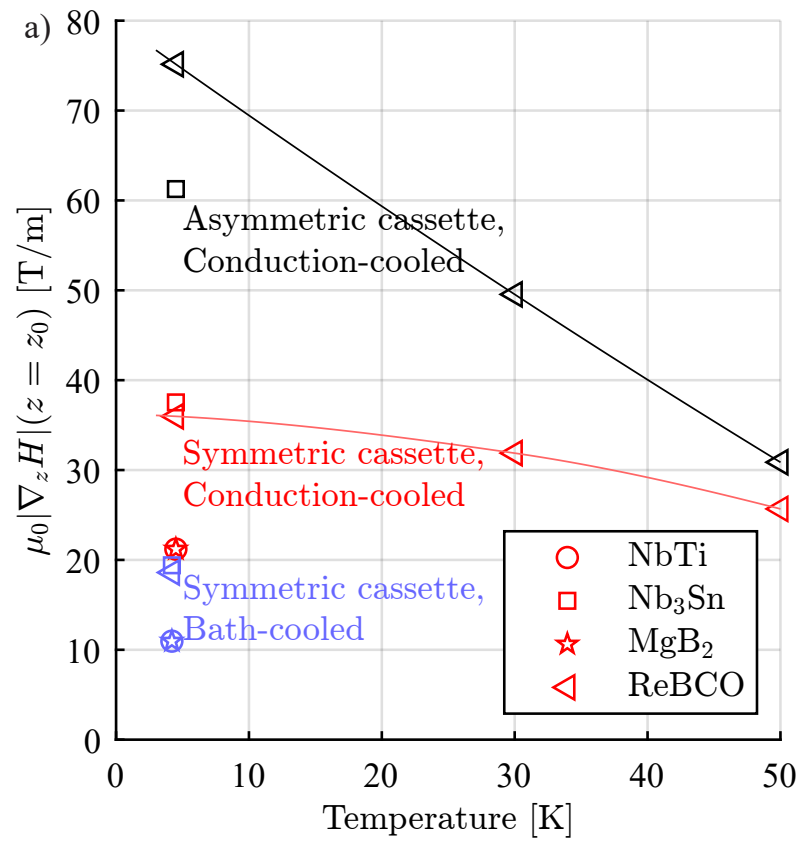

b) vacuum vessel wall

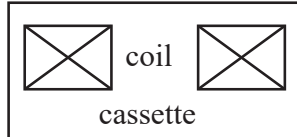

c)

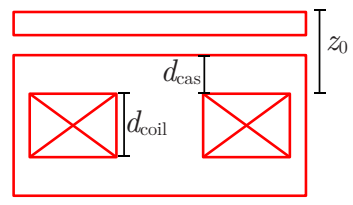

d)

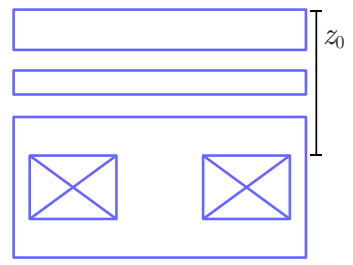

Figure 3.22: a) Estimated magnetic field gradient at the bottom of the fluid bed as a function of operating temperature. Different symbols denote different superconductors. Black markers indicate estimates using an conduction-cooled, asymmetrical cold mass design, see b). Red markers indicate conduction-cooled, symmetrical cold mass designs, see c). Blue markers indicate bath-cooled, symmetrical cold mass designs, see d). The conduction-cooled designs use a column structure to support the vacuum vessel wall whereas the bath-cooled design does not. As a result, the bath-cooled systems use thicker walls. The characteristic size of the coils $\lambda$ is set to $600 \mathrm{~mm}$. 


\subsubsection{Summary}

To conclude, the vertical magnetic field gradient $\left|\nabla_{z} H\right|\left(z_{0}\right)$ in the fluid bed of the $\mathrm{NbTi}$ demonstrator design, $20 \mathrm{~T} / \mathrm{m}$, seems to be near the maximum performance that $\mathrm{NbTi}$ can deliver using $300 \mathrm{~mm}$ wide narrow coils. A large enhancement of the vertical magnetic field gradient seems possible with ReBCO- and $\mathrm{Nb}_{3} \mathrm{Sn}$ magnets. An $80 \%$ increase is possible when using a symmetric cassette design, in which the distance between the coils and ferrofluid depends on the magnitude of the Lorentz force. The potential gains increase to a factor 4 for ReBCO and factor 3 for $\mathrm{Nb}_{3} \mathrm{Sn}$ if an 'optimistic' asymmetric mechanical support could be designed. Thus investigating such a support structure for future MDS magnets is highly recommended.

\subsection{Attraction force between coils and ferrofluid}

$\mathrm{M}^{\mathrm{s}}$ DS relies on the attraction of the ferrofluid towards the magnet. Of course, this also implies that the reaction force pulls the coils upward towards the fluid. The magnetic force between the coils and the ferrofluid has two main effects on the design of the cryostat: the coils need to be prevented from moving towards the fluid and the cryostat needs to handle the extra pressure on its surface due to the ferrofluid.

In the first part of this section an analytical expression for this extra pressure is derived. This is followed by an estimate of how ferrofluid nanoparticle sedimentation affects this pressure. Finally, a numeric model for the attraction force in a tilted MDS system is considered.

\subsubsection{Analytical expression for the coils-to-fluid force}

Consider a rectangular ferrofluid bed filled to a height $\Delta z$, as illustrated in Figure 3.23. The volumetric magnetic force density on the saturated ferrofluid at a height $z$ is given by

$$
f_{\mathrm{mag}}(z)=\mu_{0} M_{\mathrm{s}} \nabla H(z) .
$$

The magnetic field gradient in the fluid is well approximated by an exponentially decaying function, as motivated in Section 2.4

$$
\mu_{0} \nabla H(z)=-\frac{2 \pi \mu_{0}}{\lambda} H_{0} \exp \left(-\frac{2 \pi}{\lambda} z\right),
$$

where $\mu_{0} H_{0}$ is the average magnetic field at the bottom of the fluid bed, $(z=0)$. Combining this magnetic field gradient with Eq. 3.13 and integrating over the 


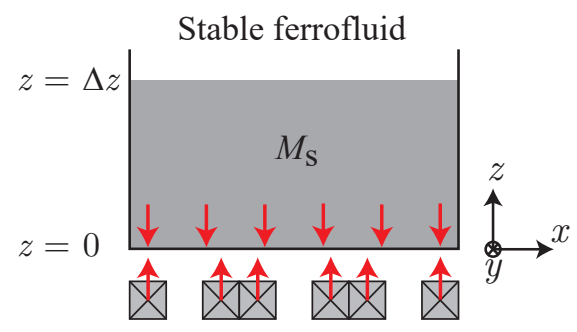

Figure 3.23: Fluid bed, filled to a height $\Delta z$, consisting of a ferrofluid with a saturated magnetization $M_{\mathrm{s}}$. The fluid bed is located above a magnet that generates a vertical magnetic field gradient. The fluid and the magnet attract each other with a force $\boldsymbol{F}_{\mathrm{mag}}$.

height of the fluid bed, yields the pressure $\boldsymbol{P}_{\mathrm{mag}}$ on the bottom of the fluid bed, due to the magnetic attraction

$$
\begin{aligned}
\boldsymbol{P}_{\text {mag }} & =\int_{0}^{\Delta z} \boldsymbol{f}_{\text {mag }}(z) \mathrm{d} z \\
& =-\frac{2 \pi \mu_{0} H_{0} M_{\mathrm{s}}}{\lambda} \int_{0}^{\Delta z} \exp \left(-\frac{2 \pi}{\lambda} z\right) \mathrm{d} z \\
& =-\mu_{0} H_{0} M_{\mathrm{s}}\left[1-\exp \left(-\frac{2 \pi}{\lambda} \Delta z\right)\right] .
\end{aligned}
$$

This equation can be rewritten in terms of the densities of the feed particles and of the ferrofluid. The density of feed particles with equilibrium height $z_{\mathrm{eq}}=0$ is set to be $\rho_{\max }$ and that of particles with $z_{\mathrm{eq}}=\Delta z$ to be $\rho_{\min }$. In this case (Eq. 2.6)

$$
\begin{aligned}
\exp \left(-\frac{2 \pi}{\lambda} \Delta z\right) & =\exp \left[-\frac{2 \pi}{\lambda} \frac{\lambda}{2 \pi} \ln \left(\frac{\rho_{\mathrm{max}}-\rho_{\mathrm{fl}}}{\rho_{\min }-\rho_{\mathrm{fl}}}\right)\right] \\
& =\frac{\rho_{\min }-\rho_{\mathrm{fl}}}{\rho_{\max }-\rho_{\mathrm{fl}}} .
\end{aligned}
$$

Eq. 2.6 with $z_{\mathrm{eq}}\left(\rho_{\max }\right)=0$ yields

$$
\mu_{0} H_{0} M_{\mathrm{s}}=\frac{\lambda}{2 \pi} g\left(\rho_{\max }-\rho_{\mathrm{fl}}\right) .
$$


Combining Eq. 3.16 and Eq. 3.17 with Eq. 3.15 yields

$$
\boldsymbol{P}_{\mathrm{mag}}=-\frac{\lambda}{2 \pi} g\left(\rho_{\max }-\rho_{\min }\right) .
$$

The attraction force scales linearly with the periodicity $\lambda$ of the current distribution and with the difference in densities of the feed particles. For the demonstrator this pressure is roughly

$$
\begin{aligned}
P_{\mathrm{mag}} & \approx-\frac{0.6 \mathrm{~m}}{2 \pi} g\left(1.13 \cdot 10^{4} \mathrm{~kg} / \mathrm{m}^{3}-1.6 \cdot 10^{3} \mathrm{~kg} / \mathrm{m}^{3}\right) \\
& \approx-9.1 \mathrm{kPa} .
\end{aligned}
$$

Here $\rho_{\max }$ and $\rho_{\min }$ correspond to the densities of lead and printed circuit boards, the heaviest and lightest constituents to be separated in shredded electronic material.

\subsubsection{Unstable ferrofluid load-case}

So far, it was assumed that the ferrofluid is saturated everywhere: $M(z)=M_{\mathrm{s}}$. However, depending on $H$ and $\left|\nabla_{z} H\right|$, it is possible for some ferrofluid compositions to become unstable: the nanoparticles gather in the high magnetic field region [47]. The force between the magnet and the fluid then increases because the average magnetic field gradient experienced by the nanoparticles is higher. In this section, the corresponding increase in $P_{\text {mag }}$ due to such an instability is estimated. Note that the system needs to be designed to handle this worst-case scenario, since it cannot be fully excluded, e.g. when the convener belt extracting sediment malfunctions.

Assume that the nanoparticles initially are uniformly distributed in the fluid, filled to a height $\Delta z$, as in Figure 3.23 and saturated with magnetization $M_{\mathrm{s}}$. When the ferrofluid becomes unstable, a significant fraction of nanoparticles sediments on the bottom. In this case, the fluid can be modelled as a combination of

(1) A ferrofluid with a high nanoparticle concentration, located between $z=$ 0 and $z=X \Delta z$, with $0 \leq X \leq 1$ (see Figure 3.24). The nanoparticles are considered to be uniformly distributed in this region, with magnetization $M=$ $M_{\mathrm{s}} / X$

(2) A non-magnetic fluid, located between $z=X \Delta z$ and $z=\Delta z$.

$X$ can be regarded as a measure of the instability of the fluid. Using Eq. 3.15, 


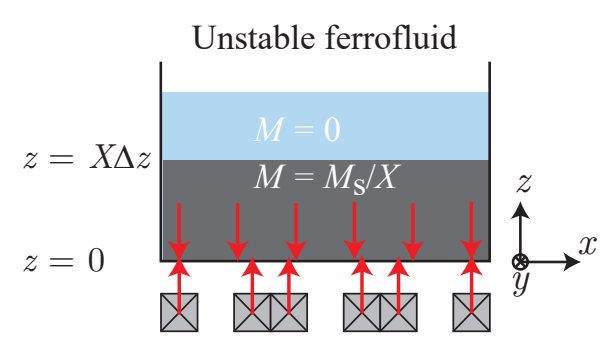

Figure 3.24: Unstable ferrofluid scenario in which the magnetic nanoparticles accumulate near the magnet. The accumulation is represented by a distance $X \Delta z$ over which the fluid is magnetized with an increased magnetization $M_{\mathrm{s}} / X$. The force between the magnet and the fluid is higher in this scenario, since the nanoparticles experience a higher average magnetic field gradient.

the new magnetic pressure compared to the stable situation can be expressed as

$$
\frac{P_{\mathrm{mag}}(X)}{P_{\mathrm{mag}, \text { stable }}}=\frac{1}{X} \frac{1-\exp \left(-\frac{2 \pi}{\lambda} X \Delta z\right)}{1-\exp \left(-\frac{2 \pi}{\lambda} \Delta z\right)} .
$$

In the limit of total sedimentation $(X \rightarrow 0)$ this becomes

$$
\begin{aligned}
\lim _{X \rightarrow 0} \frac{P_{\text {mag }}(X)}{P_{\text {mag,stable }}} & =\lim _{X \rightarrow 0} \frac{1}{X} \frac{1-\exp \left(-\frac{2 \pi}{\lambda} X \Delta z\right)}{1-\exp \left(-\frac{2 \pi}{\lambda} \Delta z\right)} \\
& =\frac{\frac{2 \pi}{\lambda} \Delta z}{1-\exp \left(-\frac{2 \pi}{\lambda} \Delta z\right)} .
\end{aligned}
$$

This worst-case pressure-increase thus depends on the initial filling level of the fluid $\Delta z / \lambda$. This relative increase (Eq. 3.21b) is shown in Figure 3.25.

For the demonstrator, an average fluid bed depth of $\Delta z / \lambda=300 \mathrm{~mm} / 600 \mathrm{~mm}$ $=0.5$ is taken (since due to the angling of the system the fluid bed is not rectangular), which means that in the case of ferrofluid instability the coil-fluid attraction force increases by a factor of 3.3 , from $9.1 \mathrm{kPa}$ to $30 \mathrm{kPa}$. Compared to the atmospheric pressure, this magnetic pressure is significant and must be included in the calculation of the required cryostat top plate thickness.

Moving a nanoparticle from far away above the magnet to the ferrofluid bottom has a larger effect than moving a particle that is already located close to the bottom. Due to the exponential decrease of the vertical magnetic field gradient the 


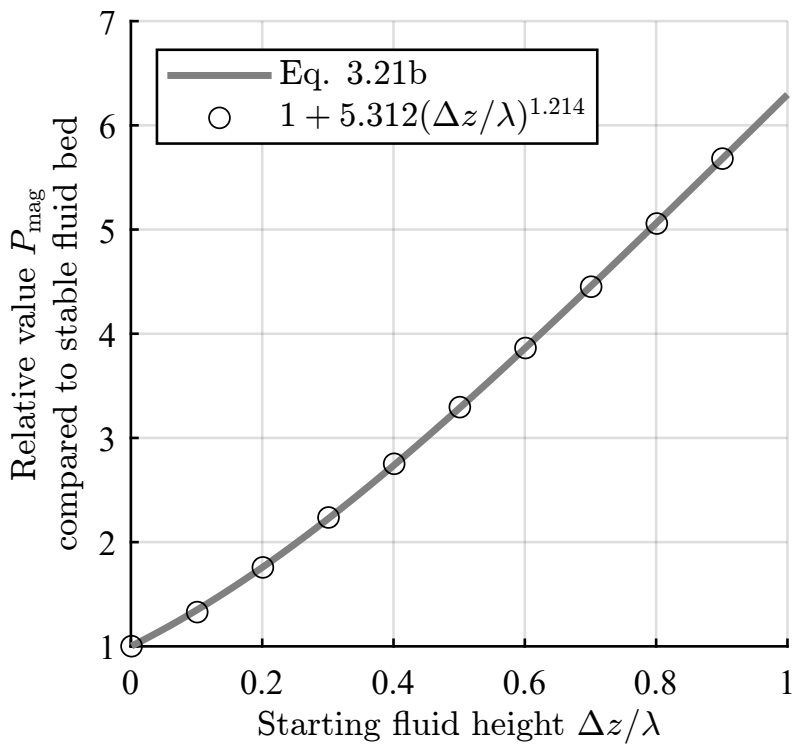

Figure 3.25: Relative increase of the coil-to-fluid force due to sedimentation of superparamagnetic nanoparticles, as a function of the relative fluid bed filling level $\Delta z / \lambda$.

relative importance of the increased distance is not very large. A fit of the form $1+5.312(\Delta z / \lambda)^{1.214}$ is shown in Figure 3.25. The growth of the extra magnetic pressure is only slight faster than linear.

Once more assuming that the fluid bed is initially filled to a height that corresponds to the equilibrium height of $\rho_{\min }$, results in the worst-case magnetic pressure as a function of the relevant variables

$$
\begin{aligned}
\boldsymbol{P}_{\text {mag,worst-case }}= & -\mu_{0} H_{0} M_{\mathrm{s}}\left[1-\exp \left(-\frac{2 \pi}{\lambda} \Delta z\right)\right] \\
& \times \frac{\frac{2 \pi}{\lambda} \Delta z}{1-\exp \left(-\frac{2 \pi}{\lambda} \Delta z\right)} \\
= & -2 \pi \mu_{0} H_{0} M_{\mathrm{s}} \frac{\Delta z}{\lambda} \\
= & -g\left(\rho_{\max }-\rho_{\mathrm{fl}}\right) \Delta z .
\end{aligned}
$$

So the worst-case pressure scales with the difference in density between the heaviest feed fraction and the ferrofluid, and with the height of the fluid bed $\Delta z$. 


\subsubsection{Numerical calculation of coil-fluid attraction for angled system}

To validate the results from Eq. 3.19b, a numerical 2D COMSOL model was used to calculate the coil-fluid attraction force for the angled three-coils MDS system. The coils are placed at a $12^{\circ}$ angle to the horizontal, as schematically indicated in Figure 3.26. The forces and torques were calculated by integration of Maxwell's stress tensor over the exterior boundaries of the domains of interest.

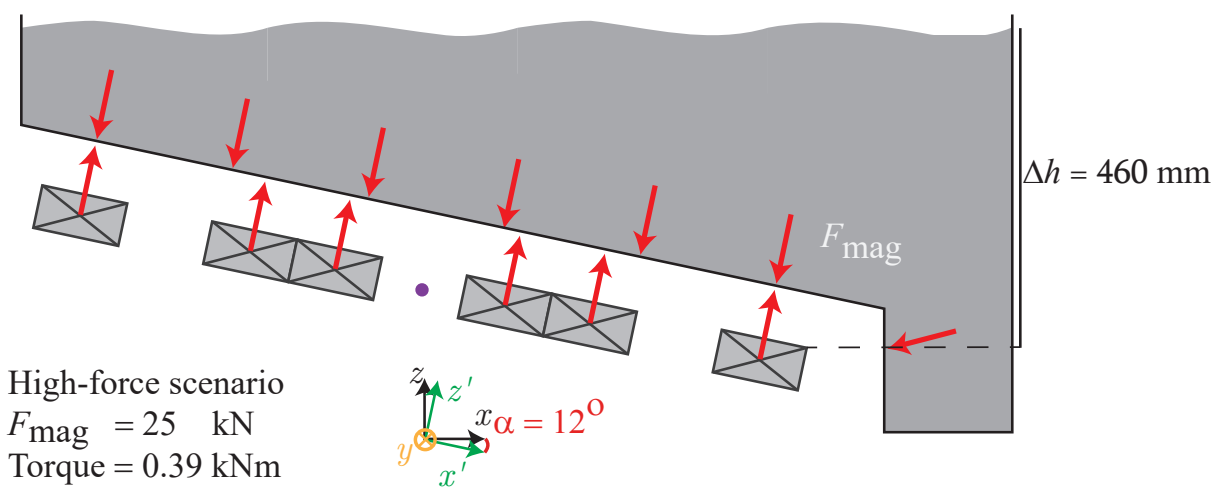

Figure 3.26: Schematic of the three coils tilted at an angle $\alpha=12^{\circ}$ with respect to the horizontal and an asymmetric fully filled ferrofluid bed. The torque on the coils is calculated with respect to the $y$-axis, indicated by the magenta dot. The vertical distance of $460 \mathrm{~mm}$ between the right-most coil and the top of the fluid bed corresponds to the maximum useable fluid bed height for this angle and this number of coils, as derived in Section 3.1.2.3.

The average magnetic field at $50 \mathrm{~mm}$ distance from the demonstrator, evaluated between $x^{\prime}=[-0.35 \mathrm{~m}, 0.35 \mathrm{~m}]$ is $2.05 \mathrm{~T}$. From this, the required magnetization of the fluid can be calculated from Eq. 3.17 to be $4.6 \mathrm{kA} / \mathrm{m}$ (for $\rho_{\max }-\rho_{\mathrm{fl}} \approx$ $10^{4} \mathrm{~kg} / \mathrm{m}^{3}$ ). The fluid's magnetization is set to this value, in the direction of the magnetic field, for regions where the magnetic field has a magnitude above $0.1 \mathrm{~T}$. For lower magnetic field regions, the magnetization is set to zero.

The distance between the top surface of the coils and the bottom of the fluid bed is set to $50 \mathrm{~mm}$ and the top of the tank is placed so that the vertical distance between the right-most coil and the surface of the fluid is $460 \mathrm{~mm}$. This corresponds to the maximum usable vertical distance for this configuration (see Section 3.1.2.3 for details). The system size in the out-of-plane direction of the fluid bed is taken to be $1 \mathrm{~m}$. This allows to convert the calculated force-per-length $[\mathrm{N} / \mathrm{m}]$ to force $[\mathrm{N}]$. 
For a full fluid tank, the calculated force between ferrofluid and coils is around $25 \mathrm{kN}$. This value includes a factor 3.3 to account for ferrofluid instability and is similar to the $28 \mathrm{kN}$ estimate from the analytical model in the previous section. Due to the uncertainties in the estimations, the mechanical design uses $125 \%$ of the highest calculated attraction force as load. The same factor 3.3 increase that was found in the analytical formula is used here, because the distribution of the nanoparticles is not simple to predict. Magnetizing the fluid at regions with magnetic fields below $0.1 \mathrm{~T}$ only increases the total force by $2 \%$.

Since the mass of the coils and their mechanical cassette is around $500 \mathrm{~kg}$, the net force that needs to be contained by the supporting structure holding the coils in place will thus be directed upwards when the fluid tank is filled and the magnet is energized. The support structure, located beneath the coils and detailed in Chapter 4.5, thus needs to be designed to handle both compressive loads (magnet turned off and/or no ferrofluid present) and tensile load (magnet energized and ferrofluid present).

Another special scenario to account for is that of an energized magnet and a partially filled fluid bed. Such a situation may occur e.g. when the liquid tank is leaking and the fluid is drained. In this case, some coils experience a strong attraction force whereas others hardly sense any attraction. The result of this is that a torque is exerted on the cold mass, as illustrated in Figure 3.27. In the case of an unstable ferrofluid, in a partially filled fluid tank, this torque can reach $1.6 \mathrm{kNm}$. The variation of the force and torque on the coils with the filling level of the fluid bed is shown in Figure 3.28.

At low filling levels, the force on the coils in the $x$-direction can be up to a factor 3.4 larger than the force in the $z$-direction. This ratio decreases to a factor 0.3 for a fully filled fluid bed.

\subsection{Conclusion}

$\mathrm{T}^{\mathrm{N}}$ this chapter the MDS demonstrator racetrack coil geometry is presented and 1 the potential performance of different types of superconductors for MDS is investigated. Two different shapes of racetrack coils were introduced, 'wide' and 'narrow' coils. When the two coil types are compared, it is found that narrow coils produce smoother magnetic field profiles and thus introduce less ripple.

An MDS magnet can be inclined to push feed particles through the ferrofluid using the horizontal component of the magnetic field gradient. For such a system, three narrow coils perform well over a wide range of tilt angles, so that a three narrow coil layout was selected for the demonstrator magnet, tilted at $12^{\circ}$. At angles of more than roughly ten degree, a 5 narrow coils configuration provides a larger effective fluid bed volume than the 3 coils system, though always well 


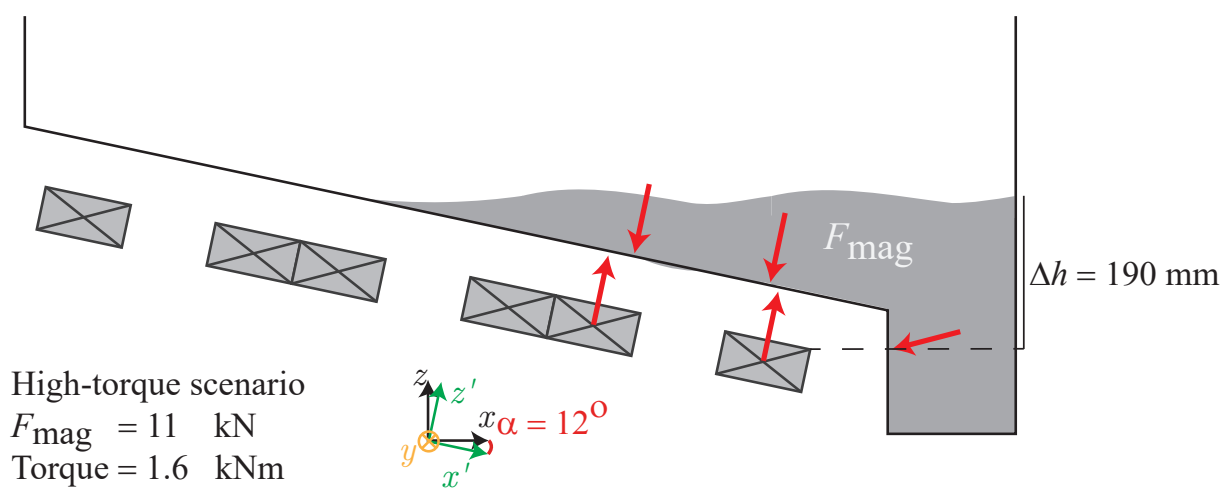

Figure 3.27: Schematic of the three coils tilted at an angle $\alpha=12^{\circ}$ with respect to the horizontal and a partially filled asymmetric ferrofluid bed. The torque on the cold mass is calculated with respect to the $y$-axis, indicated by the magenta dot. A $190 \mathrm{~mm}$ distance between the most-right coil and the top of the fluid bed corresponds to the filling level of the fluid bed that results in the maximum torque.

below a 5/3 ratio. A four-coils configuration always performs worse than the three-coils system.

The design of the NbTi demonstrator yields a vertical magnetic field gradient at the bottom of the fluid bed of $20 \mathrm{~T} / \mathrm{m}$. This is near the maximum one can expect from this specific superconductor when operating at $4.5 \mathrm{~K}$ with a $2 \mathrm{~K}$ temperature margin. $\mathrm{MgB}_{2}$ performs similarly. The potential performance of $\mathrm{ReBCO}-$ and $\mathrm{Nb}_{3} \mathrm{Sn}$-based MDS magnets highly depends on the mechanical enclosure of the coils. With the demonstrator design, a more powerful magnet is also further away from the fluid, since the Lorentz force is handled by a symmetric mechanical cassette. Such extrapolation yields an estimated performance of around $36 \mathrm{~T} / \mathrm{m}$ for both $\mathrm{ReBCO}$ and $\mathrm{Nb}_{3} \mathrm{Sn}$ operating at $4.5 \mathrm{~K}$. This value is valid for a three-coils system with characteristic size $\lambda$ of $600 \mathrm{~mm}$. If a mechanical design can be found that handles the Lorentz forces in an asymmetric way (utilizing the space below the coils so that the coils can be kept close to the ferrofluid) the performance can be greatly increased. For $\mathrm{Nb}_{3} \mathrm{Sn}$ a vertical magnetic field gradient of $60 \mathrm{~T} / \mathrm{m}$ appears possible with such a design. ReBCO at $4.5 \mathrm{~K}$ may even approach a vertical field gradient magnitude of $75 \mathrm{~T} / \mathrm{m}$. Whether such designs are cost-effective was not studied here.

The attraction force between the ferrofluid and the magnet was derived, showing that this force scales linearly with the periodicity of the current distribution $\lambda$ and with the difference in minimal and maximal densities of the feed particles. 


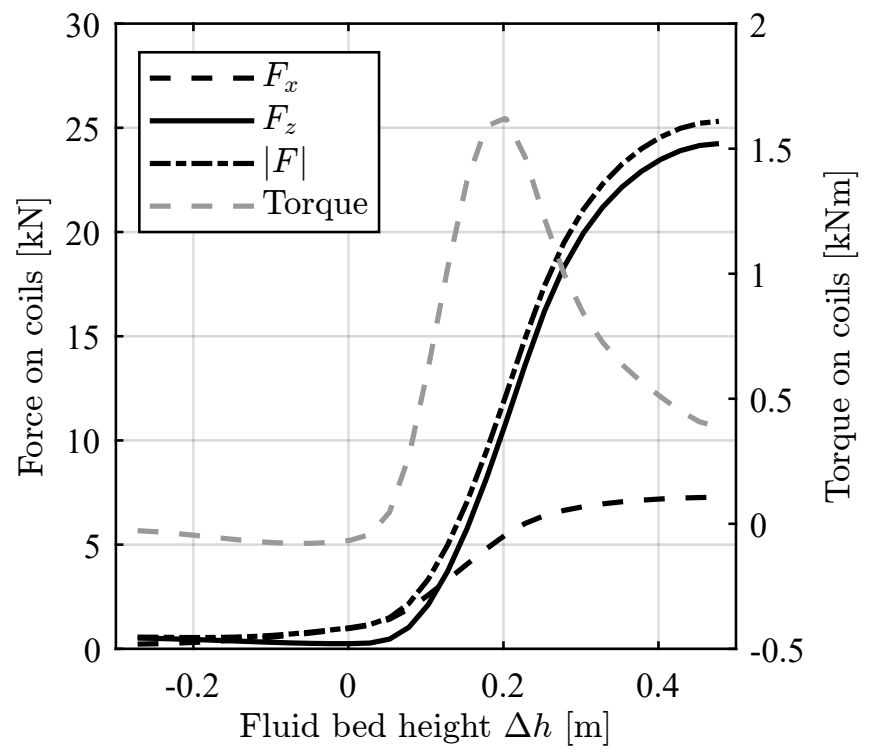

Figure 3.28: Variation of the fluid-to-magnet attraction force and of the torque with the fluid level. The total attraction force $|F|$ increases with filling level $\Delta h$. Due to the asymmetric fluid bed, a torque develops on the coils. This torque has a maximum when the fluid bed is partially filled. $\Delta h$ is defined as the vertical distance between the top of the fluid bed and the right-most point of the winding packs, see Figure 3.26.

Also the situation in which the ferrofluid becomes unstable and the nanoparticles accumulate near the cryostat wall was considered. The pressure on the top cryostat plate in such a scenario was found to be 0.3 bar. 


\section{4 | Mechanical design}

In this chapter the focus is on the different components of the system, which allow the magnet to function at nominal operating conditions. The coils require cooling to a temperature of $4.5 \mathrm{~K}$ or lower. To achieve this the coils are placed in a cryostat in vacuum and conduction-cooled by a cryocooler. In the coil windings a strong Lorentz force is generated and a structure is present around the coils to react to these. A support structure keeps the cold mass in position within the cryostat. The mechanical aspects of these components are the main subject of this chapter. 


\subsection{Introduction}

$\mathrm{T}$ the previous chapter, among others results, the required shape, size and num1 ber of coils for the demonstrator MDS magnet were introduced. This chapter concerns the design of the different components that enable the coils to function from a mechanical point of view based on the layout of the coils as derived in the previous chapter. In this chapter, the focus is on two main questions:

(1) How to handle the Lorentz force and the attraction force between coils and ferrofluid. This attraction force is estimated to be up to $34 \mathrm{kN}$ for this particular system, designed for the separation of shredded electronic material;

(2) How to minimize the coil-to-fluid distance so that the magnetic field to its fullest extent. The large planar surfaces that are formed by the bottom of the ferrofluid bed and the top surface of the coils present a unique minimization challenge that is absent in most other applications of superconducting magnets.

Perhaps the closest similar applications of superconducting magnets are wiggler and undulator magnets for accelerators [48]. Here an horizontal array of racetrack coils is also often used [49]. The main differences are that the undulator coils have a much shorter length compared to MDS, since the volume of interest is just the beam-pipe of the accelerator. Thus, more design freedom is possible for the support structure.

In this chapter the focus is on the components that primarily have a mechanical function, whereas in the next chapter thermal and electrical aspects are considered. The layout of this chapter is illustrated in Figure 4.1:

1. First, the cryostat is introduced in Section 4.2. The main function of the cryostat is to minimize heat transfer towards the coils from convection, radiation and conduction [42].To eliminate convection, the vessel is pumped vacuum. From a mechanical viewpoint, this implies that the outer vessel needs to be strong enough to withstand the outside atmospheric pressure as well as the force that pulls the ferrofluid towards the magnet.

2. Secondly, the mechanical properties of the coils are discussed in Section 4.3. Each coil consists of a winding pack, a mandrel, spacers, side-plates and end-pieces. The winding packs are anisotropic composite materials for which the mechanical properties are derived. The shape of the coils was introduced in Section 3.1.1.

3. Thirdly, the mechanical behaviour of the cold mass is considered in Section 4.4. Besides the coils, this includes their enclosure. This cassette consists of two high-strength aluminium alloy parts, that enclose the winding packs and are bolted together. During cool-down, they shrink-fit around 


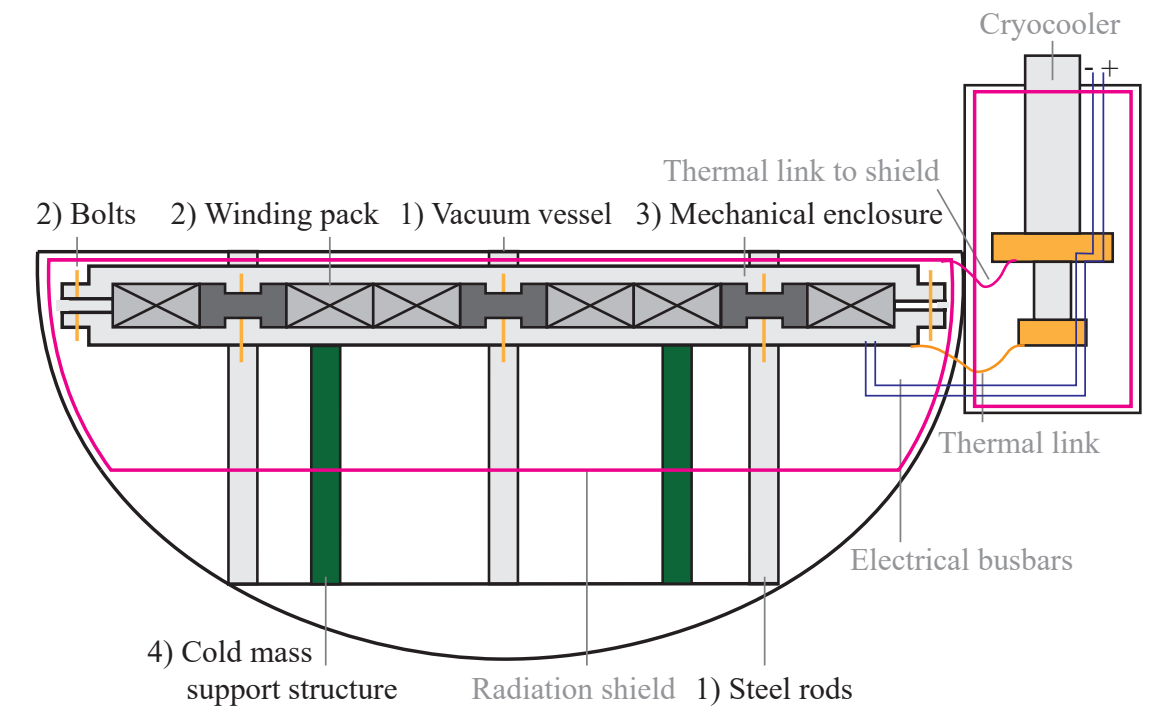

Figure 4.1: Schematic overview of components (1-4) of the magnet system as presented in this chapter. The cryocooler has been displaced relatively to the magnet for simplicity. The components indicated with grey text are presented in Chapter 5.

the coils providing pre-compression. During excitation of the magnet they react to the Lorentz force. Underneath the bottom aluminium alloy plate, several high-purity aluminium heat drains are attached to provide a high thermal conductivity link between coils and cryocooler. Thus the required mechanical- and thermal functions of the cold mass are spatially decoupled.

4. Fourthly, the support structure that keeps the cold mass in place in the cryostat is considered in Section 4.5. This structure is optimized to minimize the conductive heat load to the cold mass and to the radiation shield, while providing adequate mechanical strength. Depending on the loading situation, parts of the structure can be under compression or under tension.

Figure 4.2 gives the reader an idea of how the components are arranged in practice. As a reminder, the magnet is tilted $12^{\circ}$ relative to the horizontal plane. The coordinate system as introduced in the previous chapter is repeated here for clarity: $z$ is the vertical direction, $z^{\prime}$ is directed normal to the coil surface, $x$ and $y$ are the horizontal components whereas $x^{\prime}$ is the parallel-to-the-coil direction and lies in the $x z$-plane with an angle of $12^{\circ}$ with respect to $x$. 


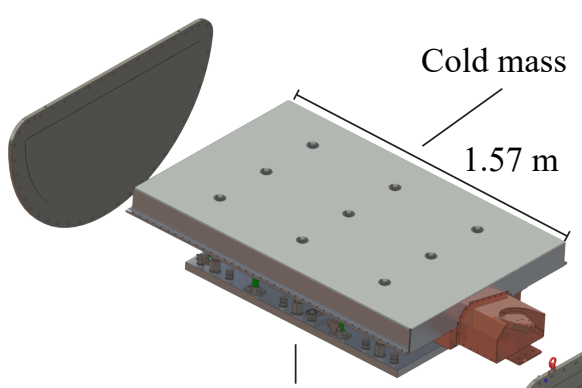

Sliding plate

$x^{\prime} z^{\prime}$-plane rotated $12^{\circ}$ clockwise to $x z$-plane, $z$ is opposite gravity

\section{Cryostat body}

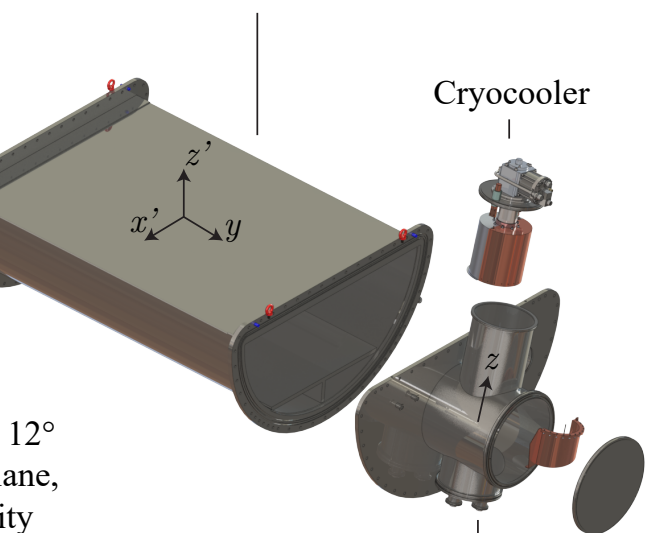

Service turret

Figure 4.2: Exploded view of main components of the magnet system.

Table 4.1 presents the thickness of the components that separate the coils and the ferrofluid of $50.5 \mathrm{~mm}$.

Table 4.1: Components determining the coil-to-ferrofluid distance.

\begin{tabular}{ll} 
Component & Layer thickness [mm] \\
\hline Cryostat top plate & 20 \\
3 MLI blankets ${ }^{\mathrm{a}}$ and vacuum & 12 \\
Radiation shield & 2 \\
1 MLI blanket and vacuum & 4 \\
Cassette & 12 \\
G11 plate & 0.5 \\
\hline Total & 50.5 \\
a These blankets take up 3 mm each when not com- \\
pressed [50].
\end{tabular}




\subsection{Cryostat}

$\mathrm{N}$ this section the design of the cryostat is introduced. The cryostat has a main 1 chamber in the shape of a half-cylinder with a flat cover plate (or D-shaped) and is illustrated in Figure 4.3.

The main vacuum vessel has a $20 \mathrm{~mm}$ thick flat plate and a $8 \mathrm{~mm}$ thick curved section. Attached to this half-cylinder is a turret housing a cryocooler, instrumentation, current leads and other services. This separate turret placement allows the cooler to work in the optimum orientation and outside the high magnetic field region, in this design below $100 \mathrm{mT}$ [51].

The cryocooler provides cooling power at two stages, a first stage typically operates between $40 \mathrm{~K}$ and $80 \mathrm{~K}$ with a cooling power of a couple tens of watt whereas the second stage has roughly 1 watt of cooling power at $4.0 \mathrm{~K}$ [52]. More detail on the thermal aspects can be found in Section 5.2.

The main chamber is made of AISI 304 stainless steel. This type of steel has a low magnetic susceptibility. The magnetic attraction of the plate towards the coils is estimated to be roughly

$$
F_{\text {mag }}=\chi V B \nabla B / \mu_{0}=2.7 \cdot 10^{-3} \cdot 0.02 \cdot 2.5 \cdot 25 /\left(4 \cdot \pi \cdot 10^{-7}\right) \mathrm{N}=2.7 \mathrm{kN} \text {. }
$$

Here the magnetic susceptibility $\chi$ of $2.7 \cdot 10^{-3}$ is representative for SS304 [42]. $V$ is the volume of the top plate and $B$ the average magnetic flux density. The value $2.7 \mathrm{kN}$ is relatively small compared to the $1 \mathrm{~atm}$ ambient pressure (i.e. $160 \mathrm{kN}$ ). Since the main chamber is at room temperature, there is no need to use the more expensive AISI 316LN stainless steel, which has a lower susceptibility than SS304 at $4 \mathrm{~K}\left(11.8 \cdot 10^{-3}\right.$ for SS316LN, up to $126 \cdot 10^{-3}$ for SS304 [53]) but similar magnetic properties at room temperature [42].

The initial concept of the MDS demonstrator assumed a helium bath-cooled NbTi magnet. However, this would have required a double-walled cryostat (ambient conditions-wall-vacuum-wall-cryogen). It was later realized that the flat top plate(s) of the cryostat would need a significant thickness to resist the combination of the outside pressure and the magnetic force from the ferrofluid-magnet interaction. To maximize the performance, i.e. the vertical gradient of the magnetic field magnitude at the fluid bed, the demonstrator design was optimized to minimize the distance between the coils and the ferrofluid.

Thus, it was decided to use a conduction-cooled magnet. Besides the enhanced performance, i.e. a higher vertical magnetic field gradient, this also saves material and reduces mass. Also, the absence of cryogens reduces the risk of injury, which is an important feature for an industrial environment. 


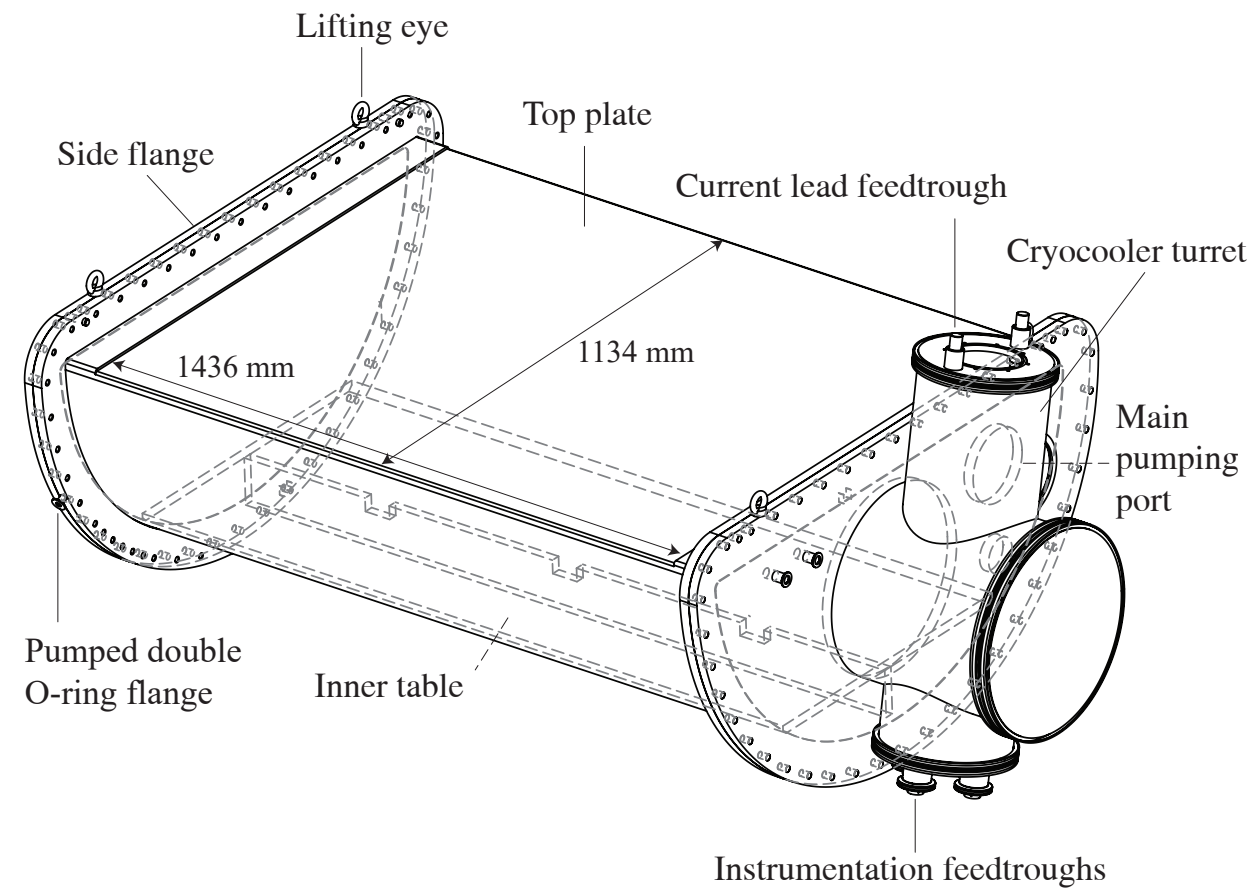

Figure 4.3: Schematic of the AISI 304 stainless steel MDS cryostat indicating the main vacuum vessel with $D$-shaped side flanges. The flanges are attached to the main chamber via double O-ring connections. One of the flanges has a turret attached to it which houses the cryocooler and instrumentation. The turret is directed vertical whereas the top plate is placed at a $12^{\circ}$ angle relative to the horizontal plane. The reason for this tilt is discussed in Section 3.1.1. Also present in the turret are the current leads. Electrical- and thermal connections are made by accessing the turret via a side-flange with diameter $400 \mathrm{~mm}$. Inside the cryostat a stainless steel table is welded parallel to the top plate. During assembly the cold mass is installed on an aluminium plate, which is then slid onto the steel table. More information on the assembly procedure is given in Chapter 6. The support structure between cryostat and the floor is not shown. 
The magnet's cold mass is supported in the main chamber by means of a structure consisting of fibreglass pillars as further detailed in Section 4.5. The cold mass rests via these pillars on a $40 \mathrm{~mm}$ thick aluminium plate which can be slid onto a table located inside the main chamber. This plate has multiple bolts that assure the parallel positioning of the coils with respect to the top plate.

To help minimize the required top-plate thickness, nine stainless steel columns are installed to support the flat top plate, thus minimizing its deflection. The columns pass through holes in the stainless steel yokes of the coils and remain at room temperature.

To verify that the displacements and stresses are acceptable, a FEM (finite element method) analysis was performed of the main chamber. The Young's modulus was set to $198 \mathrm{MPa}$ and its Poisson's ratio to 0.2895 [42]. The simulation geometry is illustrated in Figure 4.4a. The side flanges of the chamber were considered rigid and fixed in this analysis. The applied loads are 1 bar on the outside surfaces, and an extra 0.3 bar on the top plate due to fluid-to-magnet attraction, as derived in Section 3.3, as well as the reaction force $\left(3 \cdot 10^{4} \mathrm{~Pa} \times 1.1 \mathrm{~m}^{2}=34 \mathrm{kN}\right)$ on the aluminium plate where the pillars are to be mounted.

The result, shown in Figure 4.4b-d, indicate that the maximum stress remains below $100 \mathrm{MPa}^{1}$, less than half of the $240 \mathrm{MPa}$ yield strength of SS304 at room temperature [42]. The maximum stress is located at the welds between the top plate and half-cylinder. The maximum deflections of both the half-cylinder and the top plate are around $0.6 \mathrm{~mm}$. If the stainless steel column structure is omitted in the simulation, the maximum stress increases to $150 \mathrm{MPa}$ and the deflection of the top plate to an unacceptable $5.7 \mathrm{~mm}$. Thus the cryostat shall never be pumped to a vacuum without the column structure in place.

The obtained results for the cryostat without the column structure are compared to analytical results. Consider a clamped rectangular plate with sides $a$ and $b$ undergoing a uniform pressure $p$. The deflection $d z$ of the center of the plate is given by

$$
d z=\frac{12 f p b^{4}}{E d^{3}}(1-\nu)
$$

where $f$ is a scalar depending on the ratio $b / a, E$ is Young's modulus, $d$ is the thickness of the plate and $\nu$ Poisson's ratio [55]. Here $b$ is $1.426 \mathrm{~m}$ and $a$ is

\footnotetext{
${ }^{1}$ Unless specifically noted otherwise, when stress is mentioned the von Mises stress is meant. The von Mises stress is a scalar value of distortion strain energy that is often used to compare the stress state in a material with the yield strength of that material [54]. Other helpful measures of stress are the first- and third principal stresses, which indicate the maximum positive (tensile) and most negative (compressive) stress components at a location. If the first principal stress is negative, the material is under compression in every direction at that location. Likewise, if the third principal stress is positive, the material is under tension in every direction.
} 
$1.134 \mathrm{~m}$, while $p$ is set to 1.3 bar. For this ratio of $b / a, f$ is around 0.0018 . This yields a $d z$ of $5.5 \mathrm{~mm}$, very close to the $5.7 \mathrm{~mm}$ found with the FEM simulation. 
a)

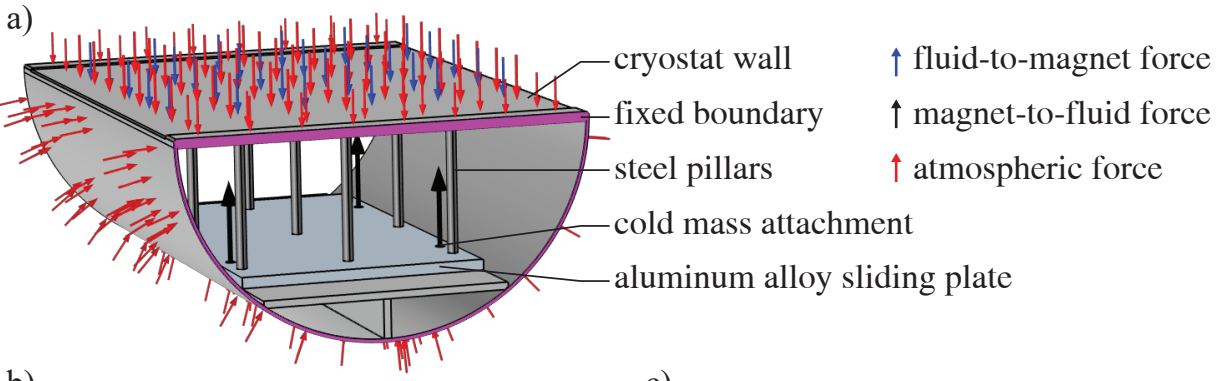

b)
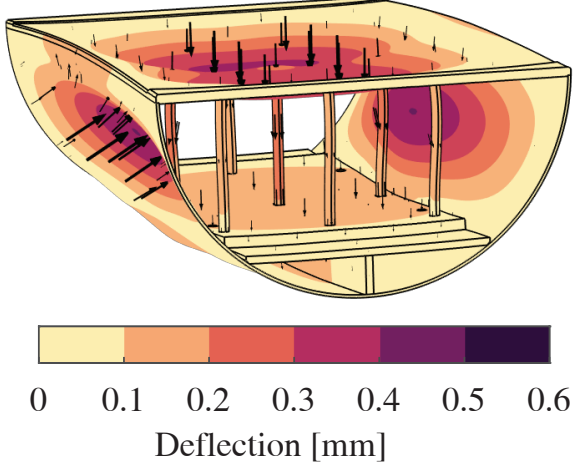

d)

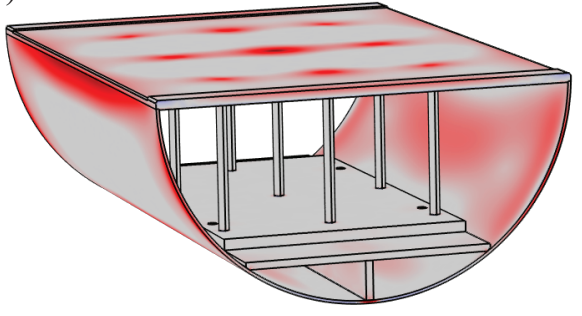

$\begin{array}{lllllllll}-80 & -60 & -40 & -20 & 0 & 20 & 40 & 60 & 80\end{array}$ First principal stress [MPa] c)

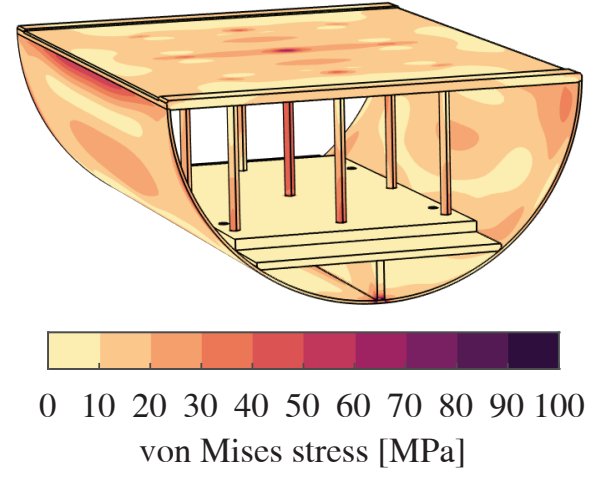

e)

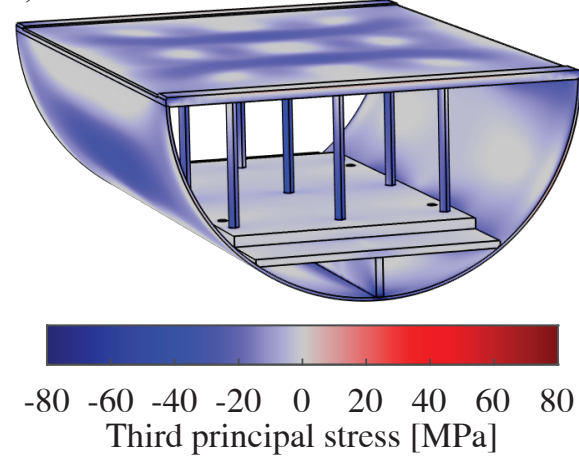

Figure 4.4: a) Schematic of the FEM analysis, indicating the main stainless steel chamber, steel support columns and the aluminium alloy inner plate. The arrows give an impression of the forces acting on the cryostat. $\boldsymbol{b})$ The deflection of cryostat chamber when subjected to atmospheric pressure and magnet-fluid force. The direction of the deflection is indicated with black arrows. The top plate and sidewall both move up to $0.6 \mathrm{~mm}$ inwards. c) The maximum von Mises stress occurs at the inner (continuous) and outer (disconnected) welds between the top plate and half-cylinder, and is lower than $100 \mathrm{MPa}$. For the outer weld the stress is mostly tensile, as indicated by the principal stress plot in $\boldsymbol{d}$ ). Compressive regions can be identified in $\boldsymbol{e}$ ), such as the inner welds and the stainless steel pillars. 


\subsection{Winding pack composite properties}

7 HIS section describes the mechanical properties of the winding pack, needed

1 for the simulation of the mechanical behaviour of the cold mass during cooldown and energizing. As discussed in Section 3.1.1, the winding pack of the coils consists of wires containing NbTi filaments, a copper matrix and electrical insulation (Formvar), embedded in epoxy resin (Stycast 2850FT blue). All these materials have different Young's moduli, yield stress and thermal expansion coefficients.

Since it is not feasible to perform mechanical 3D calculations in which every NbTi strand is explicitly modelled, mechanical properties representing the winding pack as a homogenous material are sought. For simplicity we attribute to the insulation layer the same properties as Stycast, resulting in a three-component composite. The assumption is made that the wire occupies $75 \%$ of the winding pack, an estimate that was confirmed during the winding of the coils.

Early on in the design phase, it was foreseen to use a rectangular conductor to wind the racetrack coils, since a higher packing factor is achievable and thus a better performance can be obtained [56]. The conductor was changed to a round cross-section one because it was feared that the rectangular conductor would twist during the coil winding.

Table 4.2: Mechanical properties at $4.2 \mathrm{~K}$ of materials in the winding pack.

\begin{tabular}{lllll} 
Material & $\begin{array}{l}\text { Volume } \\
\text { fraction }\end{array}$ & $\begin{array}{l}\text { Young's modulus } \\
E[\mathrm{GPa}]\end{array}$ & $\begin{array}{l}\text { Poisson's } \\
\text { ratio } \nu\end{array}$ & $\begin{array}{l}\text { Thermal expansion } \\
(\Delta L / L)_{293 \rightarrow 4 \mathrm{~K}[\%]}\end{array}$ \\
\hline Copper & 0.388 & $124[57]$ & $0.345[58]$ & $-0.324[42]$ \\
NbTi & 0.287 & $82[57]$ & $0.33[58]$ & $-0.188[42]$ \\
Stycast 2850FT & 0.25 & $24[59]$ & $0.3[60]$ & $-0.53[61]$ \\
Formvar & 0.075 & & &
\end{tabular}

Figure 4.5 shows a schematic representation of a unit cell within the winding pack. A unit cell could also be constructed from an hexagonal-close-packed winding structure, with comparable results [62].

The $\mathrm{NbTi} / \mathrm{Cu}$ conductor is capable of handling load in the longitudinal direction (indicated as (1) below) better than in the radial directions (2) and (3), which must be transferred via the Stycast. Thus the winding pack has anisotropic mechanical properties. Literature data on impregnated winding packs show that an orthotropic stress-strain relationship gives an accurate description of the behaviour of these composites [63, 64]. 


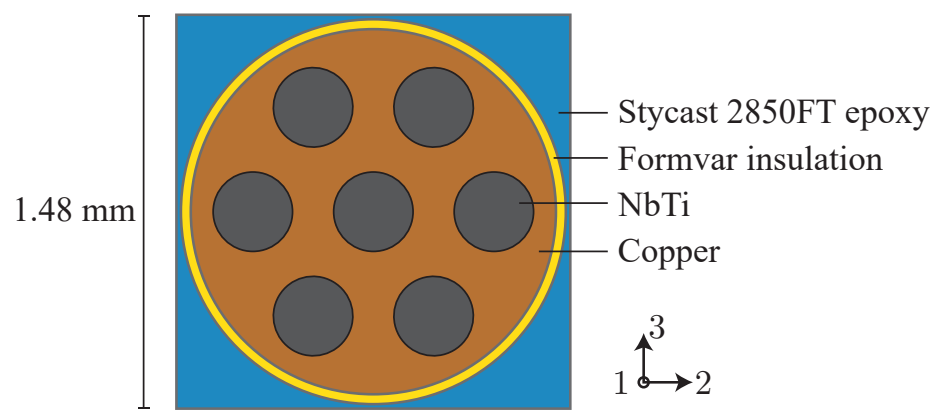

Figure 4.5: Schematic representing a unit cell of the winding pack. NbTi filaments, distributed within a copper matrix, are surrounded by an insulation layer of Formvar and Stycast 2850FT epoxy resin. The number of filaments (7) is reduced compared to that of the actual wire (132), but the volume fraction is the same.

The stress-strain relation of an orthotropic material can be written as

$$
\left[\begin{array}{l}
\varepsilon_{11} \\
\varepsilon_{22} \\
\varepsilon_{33} \\
\varepsilon_{23} \\
\varepsilon_{31} \\
\varepsilon_{12}
\end{array}\right]=\left[\begin{array}{cccccc}
\frac{1}{E_{1}} & -\frac{\nu_{21}}{E_{2}} & -\frac{\nu_{31}}{E_{3}} & 0 & 0 & 0 \\
-\frac{\nu_{21}}{E_{2}} & \frac{1}{E_{2}} & -\frac{\nu_{32}}{E_{3}} & 0 & 0 & 0 \\
-\frac{\nu_{31}}{E_{3}} & -\frac{\nu_{32}}{E_{3}} & \frac{1}{E_{3}} & 0 & 0 & 0 \\
0 & 0 & 0 & \frac{1}{2 G_{23}} & 0 & 0 \\
0 & 0 & 0 & 0 & \frac{1}{2 G_{31}} & 0 \\
0 & 0 & 0 & 0 & 0 & \frac{1}{2 G_{12}}
\end{array}\right]\left[\begin{array}{l}
\sigma_{11} \\
\sigma_{22} \\
\sigma_{33} \\
\sigma_{23} \\
\sigma_{31} \\
\sigma_{12}
\end{array}\right] .
$$

This matrix connecting strain $\varepsilon$ and stress $\sigma$ contains 9 unknowns [54]. These were estimated using the Cell Periodicity feature in COMSOL as well as with the (inverse) rule of mixtures ${ }^{2}$. The results are shown in Table 4.3 and show a good agreement between the two methods. The largest difference is found in the Poisson's ratios; here, the values obtained from the FEM model are used.

The calculated elastic modulus $E_{1}$ in the longitudinal direction is around $50 \%$ larger than that in the radial direction $\left(E_{2,3}\right)$. The calculated thermal contraction of the winding pack in the filament direction $\Delta L_{1} / L_{1}$ is roughly the same as the value for stainless steel $(-0.296 \%$ [42]), whereas in the radial directions it is in between steel and aluminium $(-0.415 \%[42])$.

\footnotetext{
${ }^{2}$ The rule of mixtures allows to calculate the effective material parameters of a composite by assuming parallel loading [63]. The inverse rule of mixtures assumes loading in series.
} 
Table 4.3: Winding pack orthotropic material properties at $4.2 \mathrm{~K}$, calculated using a FEM model of the unit cell (cell periodicity) and using the (inverse) rule of mixtures.

\begin{tabular}{lll} 
Property & Cell periodicity & Rule of mixtures \\
\hline$E_{1}[\mathrm{GPa}]$ & 76 & 79 \\
$E_{2}[\mathrm{GPa}]$ & 55 & 50 \\
$E_{3}[\mathrm{GPa}]$ & 55 & 50 \\
$G_{23}[\mathrm{GPa}]$ & 21 & 19 \\
$G_{31}[\mathrm{GPa}]$ & 18 & 19 \\
$G_{12}[\mathrm{GPa}]$ & 21 & 19 \\
$\nu_{21}$ & 0.24 & 0.21 \\
$\nu_{31}$ & 0.24 & 0.21 \\
$\nu_{32}$ & 0.30 & 0.33 \\
$\left(\Delta L_{1} / L_{1}\right)_{293 \rightarrow 4 \mathrm{~K}}[\%]$ & -0.303 & -0.304 \\
$\left(\Delta L_{2} / L_{2}\right)_{293 \rightarrow 4 \mathrm{~K}}[\%]$ & -0.349 & -0.352 \\
$\left(\Delta L_{3} / L_{3}\right)_{293 \rightarrow 4 \mathrm{~K}}[\%]$ & -0.349 & -0.352
\end{tabular}

\subsection{Mechanical behaviour of the cassette}

$\mathrm{T}^{\mathrm{N}}$ this section the mechanical behaviour of the cassette surrounding the coils is described. First the requirements it needs to satisfy are introduced, followed by a discussion of the selected design, showing how it meets these requirements. Also, some inadequate designs are shown.

The enclosure of the coils needs to fulfil several functions:

1. The coils need to be kept in place. The high current density and the strong magnetic field result in substantial Lorentz forces, as detailed in Section 3.1.2. These forces need to be contained so that the coils maintain their shape and position.

2. The magnet and the ferrofluid attract each other. The enclosure needs to prevent the coils from moving a significant distance towards the fluid.

3. The design needs to ensure that no mechanical energy release is possible with a magnitude sufficient to heat the superconductor to the normal state. 
4. The enclosure needs to provide a thermal connection between the cryocooler and the coils. This is important firstly to keep the static temperature difference between the winding pack and the cryocooler small, but also to obtain a reasonable cool-down time of the cold mass.

5. The coils are not allowed to deform significantly due to gravity.

6. The winding pack needs to be electrically insulated from the enclosure.

7. The enclosure's thickness on the top of the coils increases the distance between coils and fluid, thus decreasing the gradient of the magnetic field magnitude at the fluid bed. It is crucial to minimize this thickness.

8. The mass of the enclosure influences the required dimensions of the support structure that connects the cassette to the main body of the cryostat, and thus the conductive heat load on the cold mass. Secondly, the mass of the cassette influences the cool-down time. Over-engineering the mechanical strength of the enclosure thus has a negative effect on the thermal aspects of the system.

Some superconducting magnets require a very precise geometry because the magnetic field uniformity needs to be within very strict limits. Examples are uniform magnetic fields in the case of dipole magnets, e.g. a perpendicular oriented magnetic field to a particle beam as for the beam bending magnets in the LHC [65]; and a uniform magnetic field in the order of ppm over a well-defined ellipsoidal volume in the case of solenoidal coil systems, e.g. NMR or MRI [66]. Such ppm accuracy is not required in the vertical field gradient of MDS magnets. This is because the separation process is also influenced by factors such as poor wettability, turbulence and differences in feed particle sizes [2]. The concept of field quality in the context of MDS magnets in discussed in Section 3.1.1

The MDS demonstrator cold mass design is the result of multiple iterations. First the final geometry is introduced. Designs that were investigated but did not meet all criteria are discussed in Section 4.4.7. A schematic of the cold mass is shown in Figure 4.6. The coils are enclosed in a cassette consisting of two aluminium alloy AL5083-H321 plates, designated as top- and bottom plates, that have a thickness of $12 \mathrm{~mm}$ at the thinnest sections. This thickness increases to $24 \mathrm{~mm}$ in certain sections in order to create enough length for M10 threads in the top plate. The stainless steel yokes have an H-profile cross-section to allow for this. M10 threaded rods and nuts connect the two aluminium plates. By using the threaded rod \& nut solution the top plate of the cassette remains flat. An array of M12 bolts surrounds the cassette. Here there is space to use a bolt-and-nut configuration, see the top-left of Figure 4.6. The bolted connections are discussed in more detail in Section 4.4.5.3. 


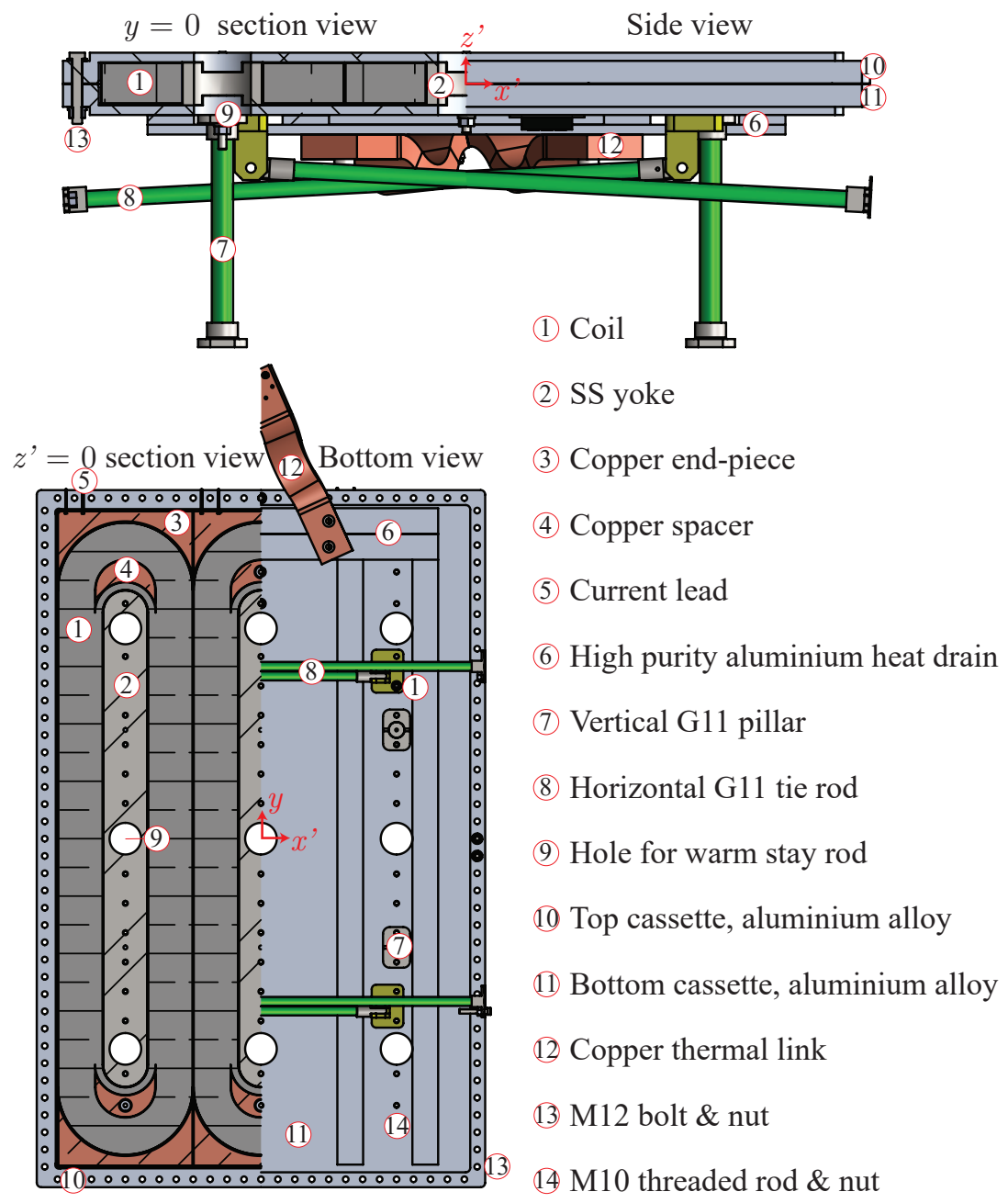

Figure 4.6: Schematic side view and bottom view of coils (1) and surrounding cassette. The cassette consists of two machined aluminium alloy plates(10,11), which are bolted together. Most of the bolts and nuts are not shown in the image. Underneath the bottom aluminium alloy part several high-purity aluminium heatdrain bars (6) reduce thermal gradients across the coils. The coils are wound around stainless-steel pole pieces (2) and have copper spacers (4) and copper end-pieces (3). Four vertical G11 fibreglass pillars (7) and four horizontal G11 tie rods (8) hold the cassette in place. Nine holes (9) in the yokes and cassette allow for the presence of room-temperature stainless steel columns (not shown). Also not shown are thin G11 plates on the top and bottom of the winding pack for electrical insulation. 
Four vertical and four horizontal G11 pillars hold the cassette in place on the sliding plate below. These pillars are optimized to balance mechanical strength with the heat in-leak through conduction. Depending on the loading scenario (room-temperature magnet, energized magnet with full fluid bed, energized magnet with partially-filled fluid bed) they can be loaded in tension or in compression. Section 4.5 deals with these pillars.

To remove the heat load on the cassette, high-purity aluminium heat drain bars with RRR $>1500$ are attached underneath the cassette using Stycast 2850FT epoxy resin. The thermal contact resistivity is not critical due to the large contact area. For more details on the thermal aspects such as thermal contact resistance the reader is referred to Section 5.2. A copper thermal link connects the aluminium bars to the cryocooler. This link is flexible to allow cool-down of the system without applying a significant mechanical load on the cryocooler due to thermal contraction.

The coils are fitted inside the cassette using stainless steel shims.

During excitation of the magnet or at normal operation a quench ${ }^{3}$ can occur due to local heating of the conductor by a release of energy. This may happen at a current well below the intended operating current [38].

As the heat capacity of materials at low temperatures is extremely small, a small amount of energy is sufficient to cause a quench [42]. Three main mechanical sources for this energy release can be identified [68]. One of these is nonelastic deformation of the conductor material. As the material yields, it heats which lets it expand locally until it work-hardens [69]. A second potential energy source is crack formation in the insulation and resin. The third is energy released by a frictional motion of the conductor due to the Lorentz force. A successful magnet design needs to take into account possible heat sources from a mechanical origin and restrict their effect. This is of special importance in conduction-cooled magnets that can not benefit from direct cooling by a liquid cryogen [38].

Non-elastic deformation of the conductor can be avoided by limiting the stress in the winding pack so that the conductor is kept in the linear stress-strain regime. By transferring the Lorentz force from the coils to the cassette over a wide contact area, local stress hotspots can be reduced.

To prevent the conductor from moving during excitation, most superconducting magnets use a glue to fill all voids between turns, so that the coil behaves as a solid object $[38]^{4}$. This movement has to be prevented because even a movement in the order of $10 \mu \mathrm{m}$ can be sufficient to initiate a quench in a NbTi magnet [68].

\footnotetext{
${ }^{3}$ A quench is a sudden, unexpected and unrecoverable transition of the superconductor from the superconducting- to the normal state [67].

${ }^{4}$ Another strategy is to allow frictionless movement of the conductor in the winding pack. By avoiding stick-slip movement, no large deposition of heat takes place [39].
} 
In this case Stycast 2850FT was selected for wet winding of the coils and as it has a thermal expansion coefficient that is relatively close to that of the conductor. Epoxy cracking can also release energy and cracks need to be avoided, which can be achieved by preventing too high tensile- and shear stresses. A clamping force is required that is larger than the magnetic force, so that the coil stays under compression. Two common strategies that are employed to achieve pre-compression are:

1. applying a pre-load at room temperature. This can, for example be done by tightening bolts and/or tension rods [70], or for solenoids, by winding a steel wire under tension around the winding pack [71]. Depending on the differential shrinking between components, part of the pretension may be lost though during cool-down;

2. utilizing materials with a larger thermal shrinkage than the winding pack. By making a cassette that can shrink-fit around the coils, compression is applied on the winding pack. This compression thus increases in magnitude during the cool-down.

Worth mentioning is the so-called bladder\&key technique, which uses an aluminium shell around the coils and hydraulic bladders and locking keys [72]. Part of the pre-stress is applied at room temperature by the keys and bladders, and part comes from differences in thermal contraction during cool-down.

The Lorentz force contributes the largest portion of the force on the coils that needs to be contained. In this chapter we introduce several tried concepts, most of which rely on the use of an aluminium alloy. As aluminium has a higher coefficient of thermal expansion than the $\mathrm{NbTi} / \mathrm{Cu}$ winding pack, the aluminium shrink fits around the coils during cool-down, providing a pre-compression force. Aluminium alloys can have much higher strength than pure aluminium, with the main downside of a lower thermal conductivity [73].

\subsubsection{Simulation set-up}

The mechanical calculations on the cold mass were performed using COMSOL Multiphysics. The simulation geometry consists of a quarter of the cold mass and is shown in Figure 4.7. The geometry represents the upper left quadrant of an $x^{\prime} y$-symmetry plane. The assumption is made that the mechanical load on this quarter in combination with symmetry boundary conditions accurately represents the whole cold mass. As a simplification the $12^{\circ}$ angle is neglected. Thus, gravity is directed perpendicular to the coils' surface (in $-z^{\prime}$ ). By considering this specific quarter of the geometry, the presence of the high-purity aluminium heat-drains running in the $x^{\prime}$-direction is amplified. 


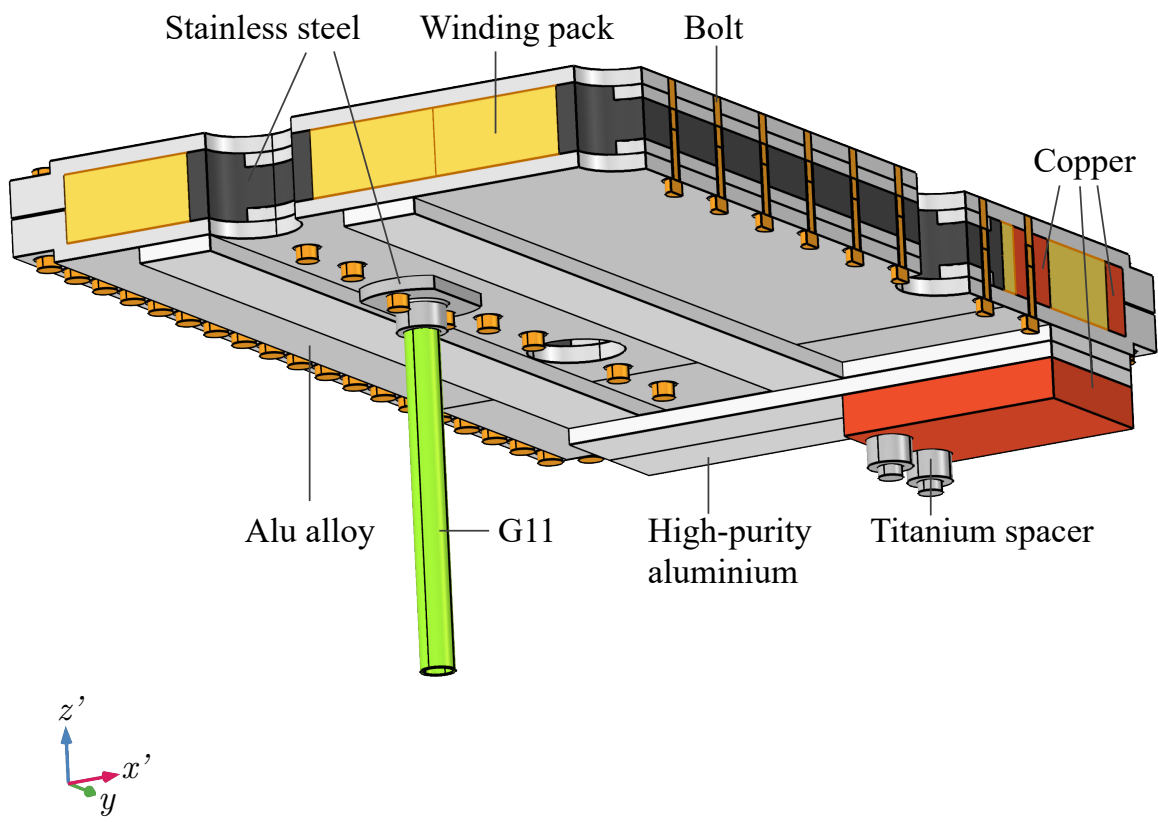

Figure 4.7: Simulation geometry, consisting of a quarter of the cold mass. Coils and stainless steel yoke are enclosed by two aluminium alloy parts, connected by stainless steel bolts. Also shown are copper crescents and end-pieces. Underneath the cassette pure aluminium heat drain bars are present, as well as a copper block representing the link between the cryocooler and the cold mass. This block is pressed to the bars by using a combination of stainless steel bolts and titanium spacers. A vertical G11 pillar is connected to the bottom cassette part via a stainless steel fixture.

In the simulation geometry only the vertical G11 pillar is considered, to verify the effect of the ferrofluid-coil attraction on the displacement of the cold mass and to analyse the glued connection between the pillar and its fixture. The horizontal G11 tie rods are assumed to have a low stiffness in the vertical direction as motivated in Section 4.5, and are not modelled in this specific simulation.

The coils are modelled as composites with homogeneous orthotropic mechanical properties, as derived in Section 4.3. To allow for this anisotropy a curvilinear coordinate system is defined with a principal axis in the direction of the current in the coils. 


\subsubsection{Contact conditions}

The components form an assembly in which relative movement of boundaries is possible depending on the settings. The boundary contact types between the different component are listed in Table 4.4. The used types of contact are

- Contact. Boundaries may have a gap between them, and when touching a contact pressure can be present. For the contact between the sides of the cassette and the outer flat sides of the winding pack, a $0.1 \mathrm{~mm}$ initial offset is put to represent imperfect shimming. For the connection between the bottom cassette and the stainless steel fixture holding the G11 pillar, a friction coefficient of 0.3 is added. This is required to prevent free motion of the pillar and fixture in the $x^{\prime} y$-plane;

- Thin elastic layer. This layer represents the thin G11 plates that are on top and bottom of each coil. The Young's modulus of this layer is set to $20 \mathrm{GPa}$ [42]. This method avoids the need to mesh the thin plates;

- Glued. The glued layer reflects the epoxy connection between boundaries; adhesion between layers takes place at room temperature for boundaries of this type as long as their initial distance is less than $0.5 \mathrm{~mm}$. The glue has an elastic modulus of $24 \mathrm{GPa}$ [59]. The main difference with the thin elastic layer boundary is that the glued boundary can break if either the tensile strength or the shear strength of the epoxy is exceeded. In the simulation the tensile strength is set to $100 \mathrm{MPa}$ at $77 \mathrm{~K}$ and $42 \mathrm{MPa}$ at $300 \mathrm{~K}$ [74]. Interpolation is set to linear and extrapolation to constant. The shear strength is similarly set to $32 \mathrm{MPa}$ at $77 \mathrm{~K}$ and $18 \mathrm{MPa}$ at $300 \mathrm{~K}$ [74].

- Continuity. This represents boundaries that can be considered fixed to each other. An example is the simplified representation of threaded connections.

\subsubsection{Material properties}

Here are presented the material properties used in the mechanical simulation of the cold mass, see Table 4.5. Most materials are modelled as isotropic. An exception is the G11 pillar, for which different thermal contraction rates are set in the radial and axial directions. The glass fibres in the resin that make up the pillar run around the tube circumference. As the pillar is relatively thin-walled, its radial contraction is close to that of the warp direction [42]. Only the $4.2 \mathrm{~K}$ values are shown, though the Young's modulus and Poisson's ratio do not change significantly with temperature. Missing in the table are the properties of the coils. These 
Table 4.4: Contact conditions between boundaries for cold mass simulation.

\begin{tabular}{lll} 
Boundary 1 & Boundary 2 & Type \\
\hline Winding pack & Yoke & Contact \\
Winding pack & Winding pack & Contact \\
Winding pack & Cassette side & Contact (0.1 mm offset) \\
Winding pack & Cassette top and bottom & Thin elastic layer \\
Winding pack & Copper crescent & Glued \\
Winding pack & Copper end piece & Glued \\
Copper end piece & Cassette side & Contact (0.1 mm offset) \\
Copper end piece & Cassette top and bottom & Thin elastic layer \\
Copper crescent & Cassette top and bottom & Thin elastic layer \\
Bolt head & Cassette & Contact \\
Bolt thread & Cassette & Continuity \\
Aluminium bar & Cassette & Continuity \\
Thermal link & Aluminium bar & Contact \\
Titanium spacer & Thermal link & Contact \\
Bolt head & Titanium spacer & Contact \\
G11 pillar & steel fixture & Glued \\
G11 pillar & Base & Fixed \\
Steel fixture & Cassette & Contact+friction \\
Bolt head & Steel fixture & Contact
\end{tabular}

are modelled as homogeneous orthotropic materials. Their mechanical properties are estimated in Section 4.3.

\subsubsection{Loads}

The following loading paths are considered in the simulations:

- First, gravity, pre-stress due to winding tension and pre-stress due to bolt tightening are applied. The whole geometry is kept at room temperature. Gravity is applied as a body load to each component. The bolt tightening is applied using COMSOL's bolt pre-stress feature. The stress related to the winding tension is detailed further below. Material properties at room temperature are used. 
Table 4.5: Mechanical properties at $4.2 \mathrm{~K}$ of materials in the cold mass. For the winding pack properties the reader is referred to Table 4.3.

\begin{tabular}{llll} 
Material & $\begin{array}{l}\text { Young's modulus } \\
E[\mathrm{GPa}]\end{array}$ & $\begin{array}{l}\text { Poisson's } \\
\text { ratio } \nu\end{array}$ & $\begin{array}{l}\text { Thermal expansion } \\
(\Delta L / L)_{293 \rightarrow 4.2 \mathrm{~K}[\%]}\end{array}$ \\
\hline Copper & $124[57]$ & $0.345[58]$ & $-0.324[42]$ \\
AL5083 & $80.9[42]$ & $0.318[42]$ & $-0.41[75]$ \\
SS304 & $210[42]$ & $0.279[42]$ & $-0.29[42]$ \\
Ti-6Al-4V & $114[42]$ & $0.404[75]$ & $-0.173[42]$ \\
G11 & $20[76]$ & $0.21[77]$ & -0.21 (warp) [42] \\
& & & -0.62 (normal) [42]
\end{tabular}

- Second, the cold mass is cooled down to $4.5 \mathrm{~K}$. This is simulated in steps so that the stress in the epoxy layer between the G11 pillar and its steel fixture can be checked for damage due to differential thermal contraction, over the entire temperature range.

- Third, the Lorentz forces are applied to the winding packs as a body force on the composite using the results of a 3D FEM magnetic field calculation. The total horizontal Lorentz forces on the quarter of the winding packs (Figure 4.7) are $489 \mathrm{kN}$ in the $-x^{\prime}$-direction and $290 \mathrm{kN}$ in $+y$, as detailed in Section 3.1.2.

- Last, the attraction force between coils and ferrofluid is applied as a body force. The worst-case scenario of $34 \mathrm{kN}$ is taken corresponding to an unstable ferrofluid in which the nanoparticles agglomerate near to the bottom of the bed [47]. In the remainder of the section, when the magnet is considered energized this means that the Lorentz force as well as the fluid attraction force are taken into account. Note that in reality the attraction force and the Lorentz force are likely to ramp up simultaneously. This is the case when the magnet is energized while the ferrofluid is present above the magnet and in the normal operation.

To find a correct method of applying the pre-stress resulting from winding the coils with a certain tension, a solenoid was first simulated, as for this case an analytical formula is available to verify the result [78]. This analytical formula allows the calculation of the hoop- and radial stresses as a function of the radial coordinate, in both the solenoid coil and the mandrel on which it is wound. The comparison between the analytical and numerical results is shown in Figure 4.8. 
The pre-stress in the COMSOL model was applied by enforcing an initial stress of $\sigma_{0}$ in the azimuthal direction. The agreement with the analytical solution was deemed sufficient to conclude that the pre-stress on the racetrack coils and the stainless steel yokes can be approximated by applying an initial stress, determined by the winding tension, in the direction of the wire. The same curvilinear coordinate system used to apply the anisotropic material properties is used.

Also taken into account is the stress resulting from pressing on the coil during curing of the Stycast in the manufacturing process. This pressure is exerted on the straight section of the racetracks and is estimated at $5 \mathrm{MPa}$. More details on the winding of the coils can be found in Section 6.2.

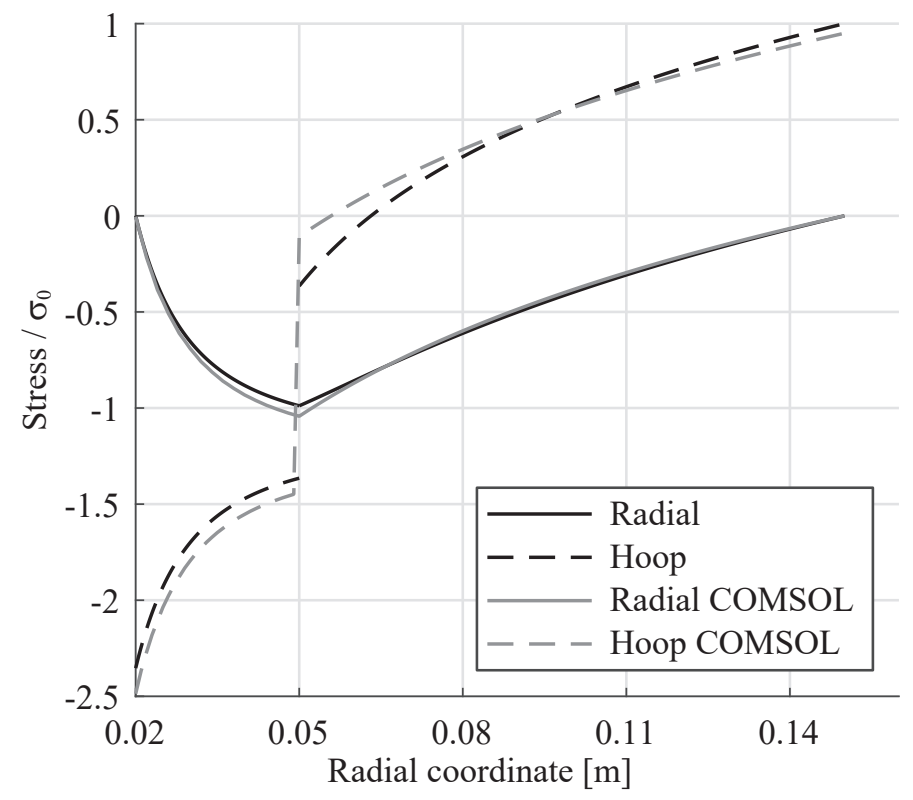

Figure 4.8: Hoop- and radial stresses in a solenoid wound with a pre-tension $\sigma_{0}$ on a mandrel. The mandrel in this example has an inner radius of $0.02 \mathrm{~m}$ and outer radius $0.05 \mathrm{~m}$. The coil has an inner radius of $0.05 \mathrm{~m}$ and outer radius $0.15 \mathrm{~m}$. The figure shows the stresses computed using an analytical formula from [78] as well as results from a COMSOL 2D axisymmetric simulation. 


\subsubsection{Simulation results}

\subsubsection{Aluminium alloy cassette}

The largest stress in the aluminium cassette is situated around the holes in the cassette for the room-temperature stainless steel columns that support the cryostat top plate. The von Mises stress in the cassette after energizing is shown in Figure 4.9. The peak stress increases from $174 \mathrm{MPa}$ after cool-down to $197 \mathrm{MPa}$ after applying the Lorentz force. This is around $72 \%$ of the yield stress of the alloy at 4.2 K (275 MPa [73]) and slightly higher than the factor $2 / 3$ initially aimed for. The $197 \mathrm{MPa}$ is $34 \%$ of the $572 \mathrm{MPa}$ ultimate tensile strength of AL5083 H321 at $4.2 \mathrm{~K}$ [79].

Whereas local plastic yielding of the cassette could in principle be allowed, the gain in performance, that is of the vertical magnetic field gradient at the fluid bed, by reducing the cassette thickness is small. For a more powerful magnet, i.e. one that generates higher Lorentz forces, a more aggressive cassette design (i.e. allowing local plastic yielding) may be beneficial. However, reducing the cassette thickness would also reduce the applied compression on the coils during cool-down, as further discussed in Section 4.4.6.

The high-purity aluminium bus bars see a stress of $40 \mathrm{MPa}$. Since the yield strength of pure aluminium is as low as $35 \mathrm{MPa}$ [42], some plastic yielding of the bars can occur. This non-linear stress-strain relation is not taken into account in the model. The maximum stress in the Stycast layer between the bars and cassette is below $30 \mathrm{MPa}$, well below the yield strength [74].

A question that arises in view of the stress concentration around the holes is whether using such a column structure is indeed preferred.

The deflection of the cryostat top plate scales with the top plate thickness as $d^{-3}$, as seen in Eq. 4.2. In combination with the results from Section 4.2, it is estimated that the cryostat top plate, if unsupported by columns, needs a thickness of $43 \mathrm{~mm}$ to obtain the same deflection as a $20 \mathrm{~mm}$ supported plate. Thus the gain of using the column structure for the cryostat thickness is at least $23 \mathrm{~mm}$ whereas the cassette is only $12 \mathrm{~mm}$ thick. The issue of whether these columns also make sense for higher-field MDS magnets is addressed in Section 4.4.6.

\subsubsection{Winding pack}

The computed displacements of the winding packs are shown in Figure 4.10a-c in the directions $x^{\prime}, y$ and $z^{\prime}$. Three scenarios are presented: (1) the initial roomtemperature situation including winding pre-tension, gravity and tightening of the bolts, (2) cool-down to $4.5 \mathrm{~K}$, and (3) energizing the magnet with a ferrofluid present above it. The shrinkage of the cold mass in the $x^{\prime}$-direction shows almost 


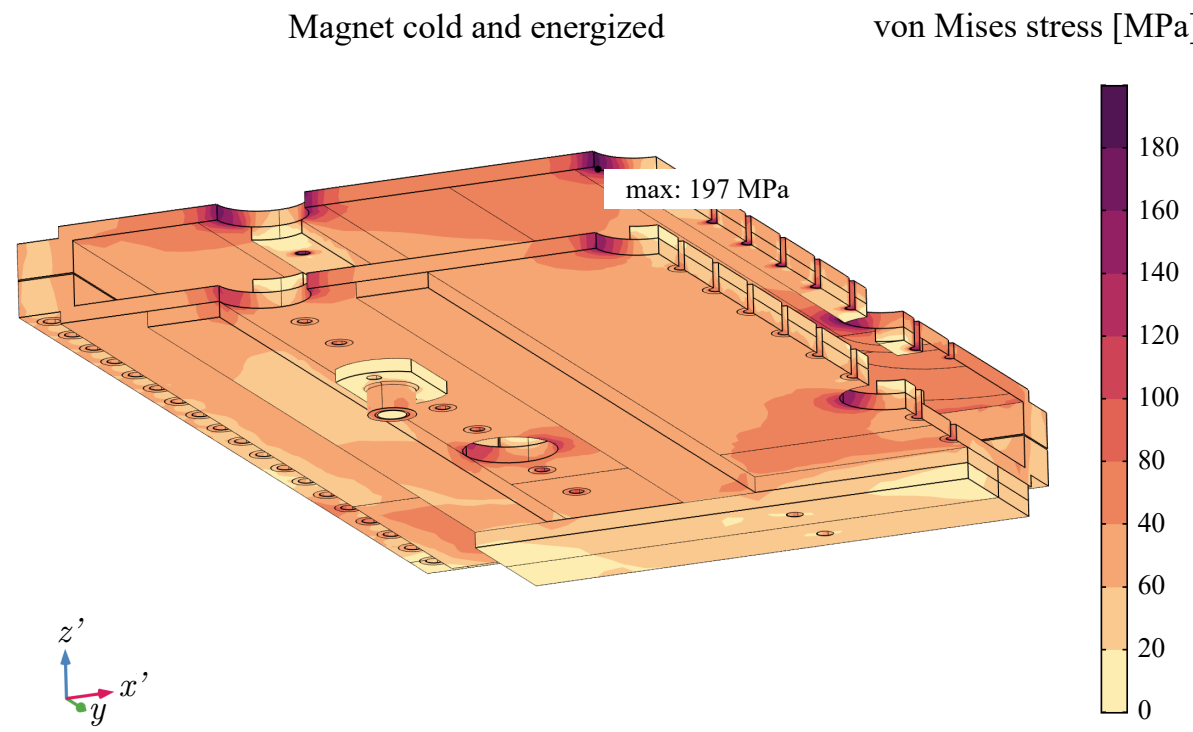

Figure 4.9: Von Mises stress in the aluminium cassette after cool-down of the cold mass and energizing.

no variation with the $y$-direction. Likewise the displacement in $y$ shows almost no variation in $x^{\prime}$. Thus it is concluded that the aluminium cassette behaves as a rigid body during the shrink-fit.

The relative displacement of the winding pack in the $x^{\prime}$-direction towards the origin is $0.369 \%$, in the $y$-direction $0.353 \%$. It makes sense that the shrinking in the $y$-direction is somewhat less when considering that the transverse thermal expansion of the unconstrained winding pack is $-0.352 \%$ compared to $-0.304 \%$ in the longitudinal direction (Table 4.3). The calculated values of the constrained winding pack are in between those of the unconstrained winding pack and of the empty cassette $(-0.415 \%)$.

In the $z^{\prime}$-direction the cold mass moves downwards because the G11 pillars to which the cold mass is attached are fixed at the bottom. The magnitude of this displacement is exaggerated because the whole pillar is cooled to $4.5 \mathrm{~K}$ in this simulation. In reality one side of the pillars is attached to the room-temperature cryostat whereas the other is attached to the $4.5 \mathrm{~K}$ cold mass. A 1D FEM calculation estimates the heat-sinked vertical pillars to shrink $0.5 \mathrm{~mm}$.

As for the variation of the $z^{\prime}$-displacement with the $x^{\prime}$ - and $y$-coordinates, some bending $(0.6 \mathrm{~mm})$ of the coils takes place. This is the consequence of the presence of the aluminium bars on the bottom of the cassette that allow the bottom cassette to pull on the winding pack harder than the top cassette. 
During the energizing of the magnet the Lorentz force tries to expand the coils. In the $x^{\prime}$-direction the cold mass is rigid enough so that no large deformation takes place. The same holds for the displacement in the $y$-direction. In the $z^{\prime}$-direction, however, the coils moves half a millimetre towards the ferrofluid. This counteracts the expected movement during cool-down.

The von Mises stress in the winding packs is shown in Figure 4.10d. The maximum ( $89 \mathrm{MPa}$ ) is found in the inner section of the heads after cool-down. This decreases to $83 \mathrm{MPa}$ after the magnet is turned on.

The different stress components are shown separately in Figure 4.11. From Figure 4.11a it can be seen that a tensile longitudinal stress is present at room temperature, caused by the winding tension. After cool-down this stress component mostly disappears due to the shrink-fit of the cassette. When energizing the magnet the longitudinal stress increases, with a maximum at the inner radius of the bent sections of up to $40 \mathrm{MPa}$. Assuming parallel loading of the composite in the direction of the current, the rule of mixtures can be applied to estimate the longitudinal stress in the different components of the winding pack. This leads to an estimated $12 \mathrm{MPa}$ tensile stress in the epoxy, which is well below the yield strength in tension of more than $100 \mathrm{MPa}$ of Stycast 2850FT at $4.2 \mathrm{~K}$ [74]. $\mathrm{NbTi} / \mathrm{Cu}$ superconductor can handle up to $500 \mathrm{MPa}$ in tension before a significant degradation of the superconducting properties occurs [39] so that a large safety margin is present.

In the transverse direction ${ }^{5}$, see Figure $4.10 \mathrm{~b}$, the winding pack sees an initial small compression at room temperature due to the winding tension, and a large increase in compression during cool-down. When the magnet is energized part of the compression is relaxed. The magnetic forces are not sufficient to cause a positive transverse stress in the composite winding pack. A negative transverse stresses reduces the formation and propagation of cracks in the epoxy, and the release of mechanical strain in the form of heat that is associated with these cracks, and is thus required [38] for LTS magnets. Thus the aluminium cassette is thick enough as far as the pre-compression is concerned.

The maximum shear stress at the interface between coils and the stainless steel yokes, see Figure $4.11 \mathrm{c}$, is $7 \mathrm{MPa}$. This shear stress would be higher if this connection would be glued. A Teflon coating applied to the steel yoke before winding allows for the free relative movement of the components. The copper crescent spacers, see Figure 4.6, are however glued in the winding pack and a shear stress of up to $14 \mathrm{MPa}$ develops in the outer heads of the winding packs at this interface. This remains below the epoxy's shear strength of $32 \mathrm{MPa}$ at $77 \mathrm{~K}$ [74].

The coils stay in contact with the steel yokes after energizing. The maximum

\footnotetext{
${ }^{5}$ Taken here as the direction perpendicular to the conductor in the $x^{\prime} y$-plane.
} 
a) Displacement in $x^{\prime}[\mathrm{mm}]$

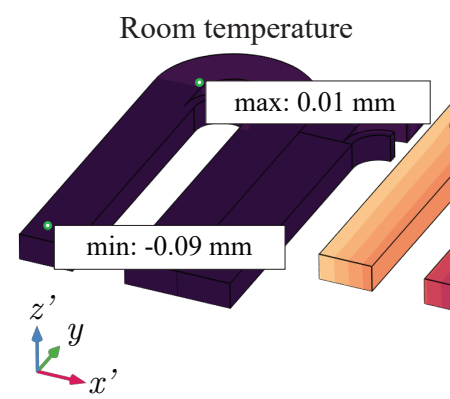

Cool-down $\quad$ Energized + attraction

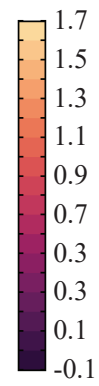

b) Displacement in $y[\mathrm{~mm}]$
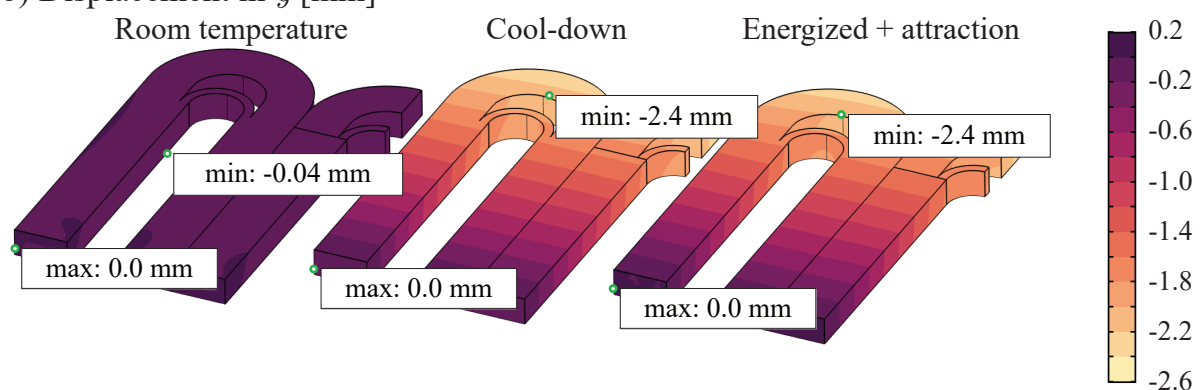

c) Displacement in $z^{\prime}[\mathrm{mm}]$
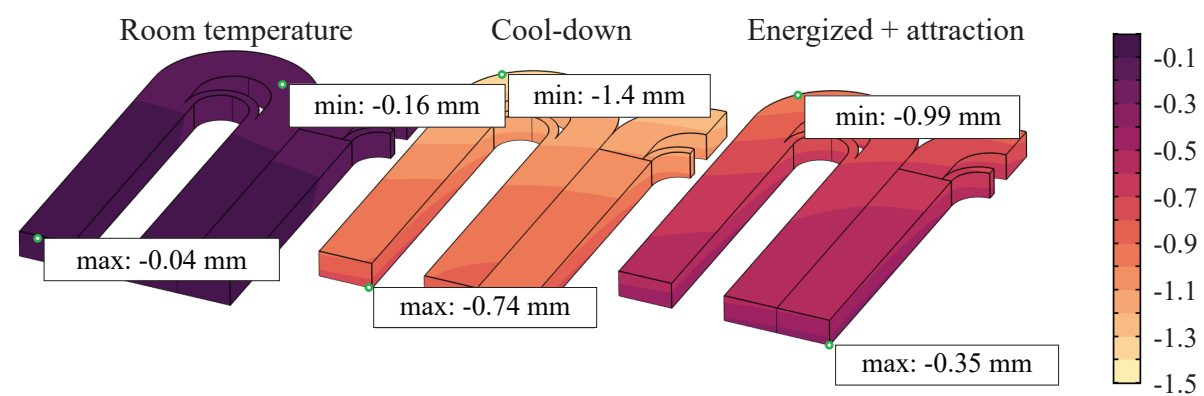

d) von Mises stress $[\mathrm{MPa}]$

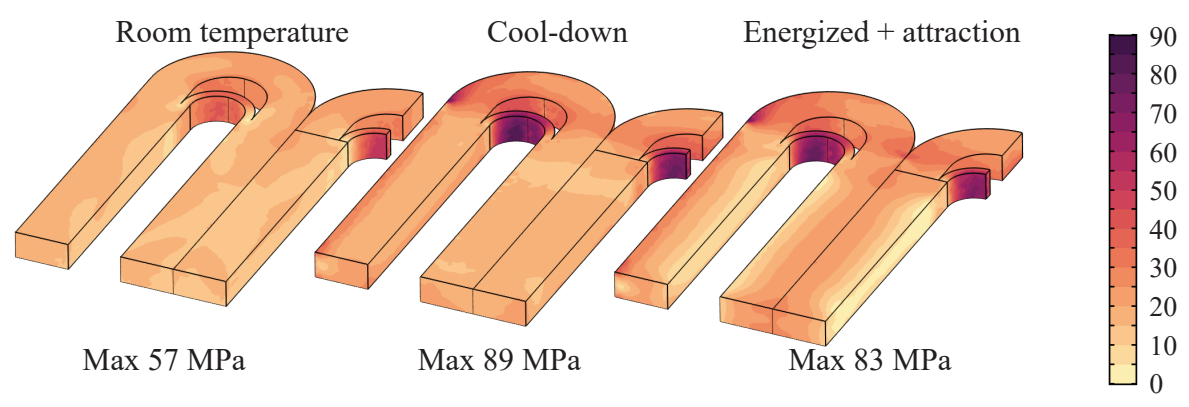

Figure 4.10: Simulation results of the displacement of the winding packs. $\boldsymbol{a}, \boldsymbol{b}$ and $\boldsymbol{c}$ refer to the displacements in directions $x^{\prime}, y$ and $z^{\prime}$ respectively; $\boldsymbol{d}$ shows the von Mises stress. 
contact pressure of $85 \mathrm{MPa}$ occurs after cool-down, see Figure 4.11d, and reduces by around $20 \mathrm{MPa}$ after excitation.

Here we so far neglected the effect of thermal stress within the composite. Stycast 2850FT and other epoxy resins are brittle at cryogenic temperatures [38]. As the winding pack's components have different thermal expansion coefficients, thermal stress will develop during cool-down of the coils. This is generally of sufficient magnitude to cause cracks in the epoxy [62]. The formation of cracks can relieve the stress concentration, however, the cracks can also act as sites for the initiation of further cracks when the magnet is energized. The release of energy associated with crack formation and crack propagation is large enough to quench NbTi magnets [62].

It is hard to predict the training behaviour of the magnet, that is, how many quenches will occur before the magnet is able to reach its nominal magnetic field strength. However, crack formation does not seem prohibitive to eventually reaching the design magnetic field. A positive side-note on the expected training behaviour is that the magnet is designed to operate at the low fraction of $16 \%$ of the short- sample critical current, whereas magnet training usually causes problems when trying to operate closer to the short sample limit $[68,69]$.

\subsubsection{Clamping the coils - bolts \& threaded rods}

Next we focus on the simulation of the cold mass as a whole. The two cassette parts need to be attached to each other in order to keep the coils in place and to maintain contact. This is achieved by using an array of 128 stainless steel M12 bolts surrounding the coils, and by 48 stainless steel M10 threaded rods that run along the $y$-centreline of each coil. Figure 4.12a shows a schematic representation of the bolts and threaded rods. To minimize the required computational effort, the geometry is simplified as shown in Figure 4.12b.

Perhaps counter-intuitively, the tensile load on the bolts and threaded rods increases significantly during cool-down, from $30 \mathrm{MPa}$ to $130 \mathrm{MPa}$ depending on the bolt location. This is caused by the higher thermal expansion coefficient of the aluminium alloy compared to the winding pack (aluminium shrinks $-0.415 \%$, stainless steel $-0.296 \%$ and the winding pack -0.303 to $-0.349 \%$, see Section 4.3). In other words, the cassette "wants" to shrink more than the coils. Thus, the outer parts of the cassette are pulled apart, as illustrated in Figure 4.12c. The bolts keep the two cassette parts together, resulting in the increased tensile stress on the bolts, see Figure 4.12d. Since the length-scales of interest in the $x^{\prime} y$-plane are much larger than the length of the bolts, differential thermal shrinking in the $z^{\prime}$-direction does not play a significant role.

The stress in the bolts is shown in Figure 4.13. To ensure that the bolts 
a) Longitudinal stress [MPa]

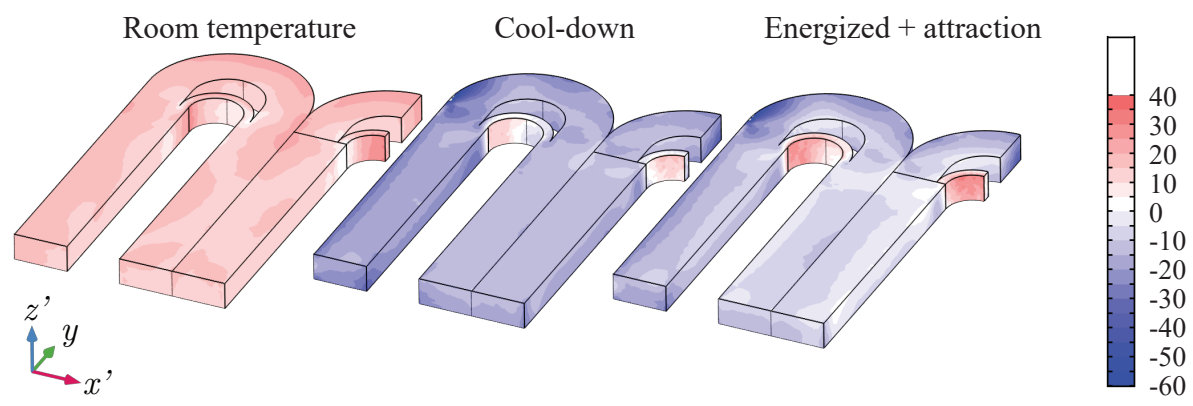

b) Transverse stress $[\mathrm{MPa}]$

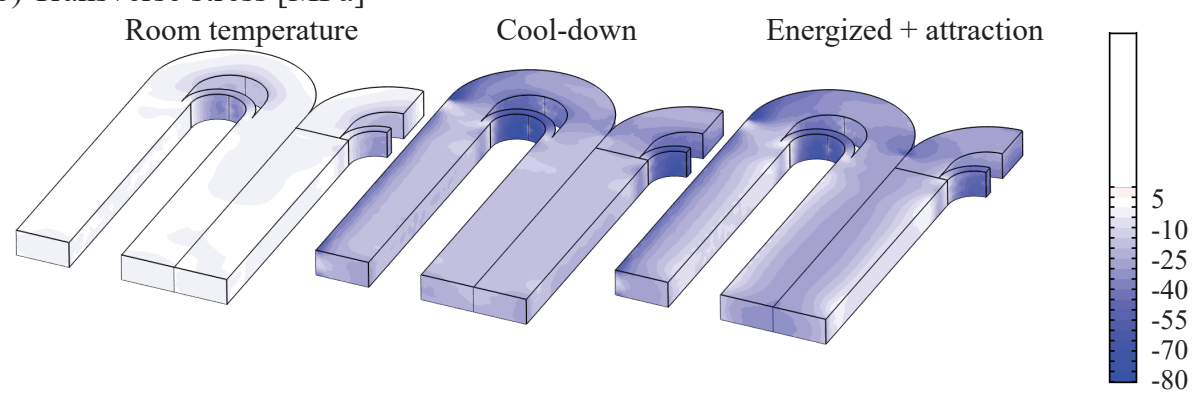

c) Shear stress [MPa]

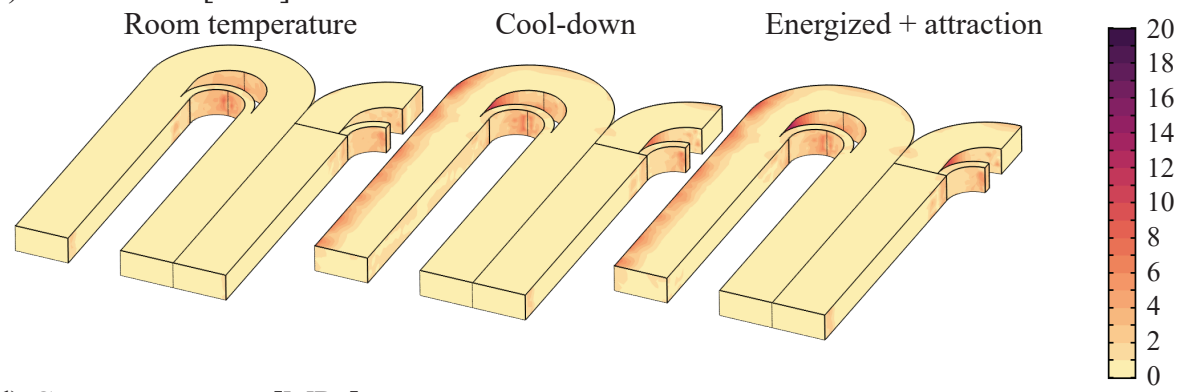

d) Contact pressure $[\mathrm{MPa}]$

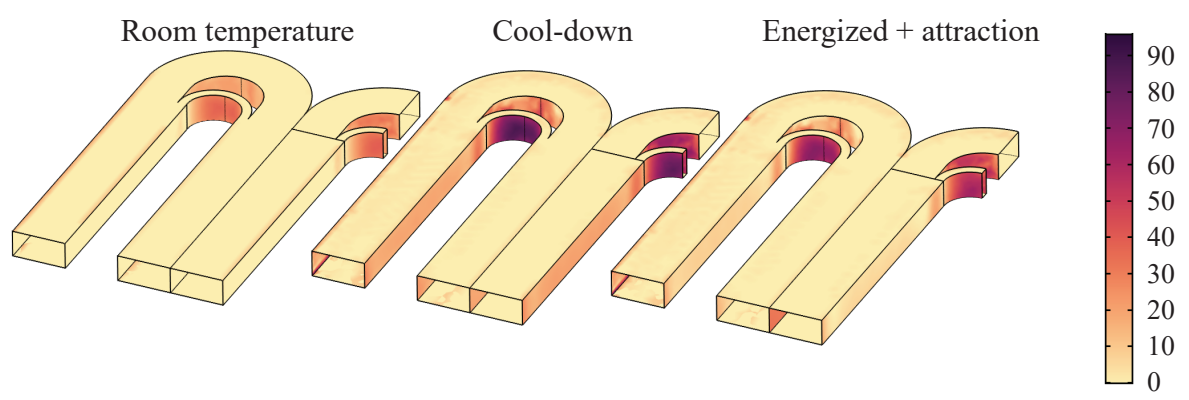

Figure 4.11: Simulation results of stresses in the winding packs: a) Longitudinal stress; b) Transverse stress; $\boldsymbol{c}$ ) Shear stress. $\boldsymbol{d}$ shows the contact pressure. 

a) Clamping design
b) Simulation geometry
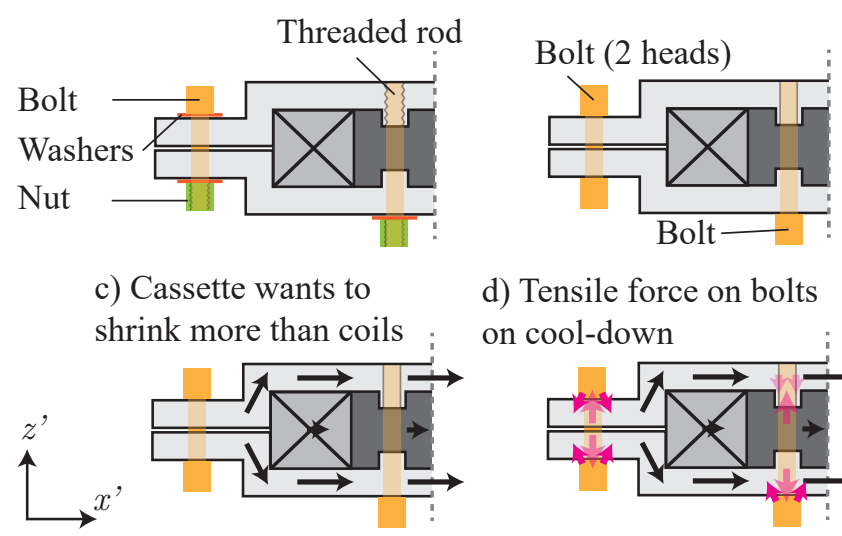

d) Tensile force on bolts on cool-down

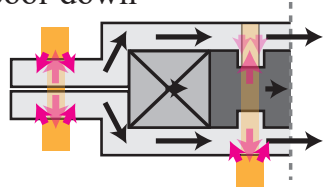

Figure 4.12: a) Schematic of the method used to fix the cassette to the coils. Around the cassette, bolts and nuts keep the two aluminium alloy parts together. Through the yokes of the coils threaded rods are present, fixed in threads in the top cassette and by nuts underneath the bottom cassette. b) This geometry is simplified in the mechanical simulations; the outer bolts and nuts are modelled by bolts with two heads. The threaded rods are represented by a bolt, fixed to the top cassette by a simplified thread. The washers are represented by increasing the bolt head thickness. c) During cool-down the two cassette halves try to pull themselves away from each other, as they shrink towards the $x^{\prime}$-symmetry plane. d) This results in a tensile load on the bolts.

see approximately the same load during cool-down, a pre-stress is applied to the bolts at room temperature of around $20 \mathrm{MPa}$. However, the bolts connecting the thermal link to the cassette are pre-stressed to $160 \mathrm{MPa}$ as the clamped connection between the thermal link and the cassette benefits from a high force. This link and the interface with the cold mass is discussed in more detail in Section 5.2.

Titanium spacers are used between the bolt heads and the thermal link to ensure that this pre-tension is not lost during cool-down, since the stainless steel bolts have a relatively low thermal contraction coefficient. Since titanium has a thermal contraction coefficient even lower than that of stainless steel [42], nevertheless the bolts remain in tension.

\subsubsection{Effect of cassette thickness}

In this section the influence of the cassette thickness is presented on (1) the transverse compression of the winding pack during cool-down, (2) the longitudinal 


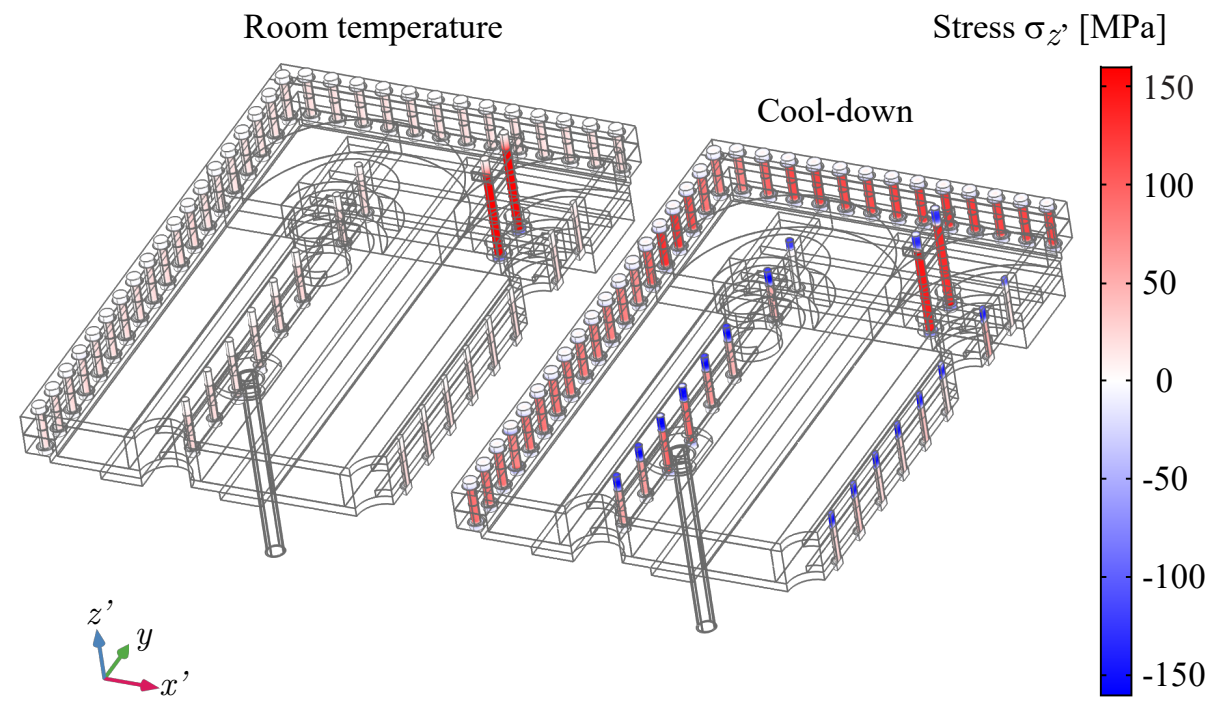

Figure 4.13: Stress in the bolts in longitudinal direction $z^{\prime}$ after pre-tensioning at room temperature and after cool-down.

stress in the winding pack and (3) the stress in the cassette itself. All these are obtained from COMSOL simulations.

The geometry of the cold mass is simplified to keep the calculation time acceptable. It consists of an octant of the geometry, see Figure 4.14. The aluminium cassette is simplified to a single solid piece. The material properties of the components are the same as in the previous sections. The dimensions of the coils are that of the MDS demonstrator (see Section 3.1.4). Perfect shimming of the coils in the cassette is assumed, i.e. there is no offset between boundaries at roomtemperature. The effect of winding tension, which is beneficial for the transverse stress and unwanted for the longitudinal stress, is not considered.

The longitudinal stress is evaluated in point A in Figure 4.14, since it is the highest at this location and also the magnetic field is highest here. The transverse stress is evaluated at point B. The magnetic field is of similar magnitude at this point.

A parametric study is performed where the thickness of the aluminium alloy cassette is swept from 12 to $100 \mathrm{~mm}$. For each thickness, a simulation is performed that starts with a calculation of the thermal stress, followed by applying a Lorentz force of various magnitudes. The electromagnetic forces range from 0.5 to 8 times the Lorentz force level experienced by the NbTi demonstrator during normal operation, $F_{\mathrm{L} 0}$. 


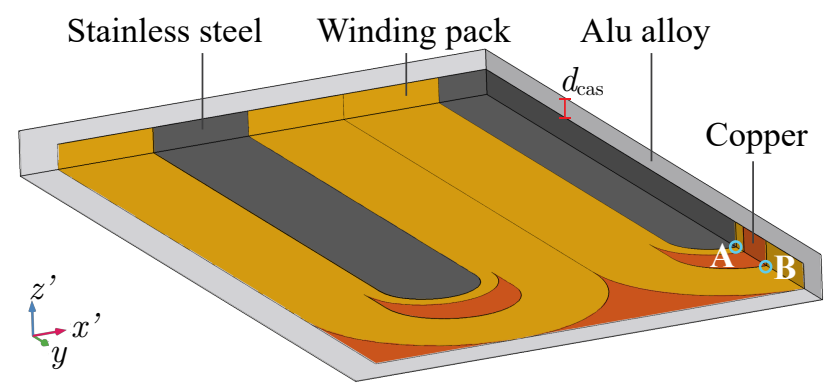

Figure 4.14: Simulation geometry, consisting of an octant of the cold mass. The copper pieces and winding packs are glued together. The winding pack and the aluminium have a contact boundary condition.

In Figure 4.15 the transverse stress in the winding pack is presented as a function of the cassette thickness. The stress is negative after cool-down due to the differential thermal contraction and increases in magnitude with increasing cassette thickness. The Lorentz force increases this stress, with a magnitude that seems to be fairly independent of the cassette thickness. It was chosen to maintain a negative (compressive) transverse stress to prevent tension on the epoxy, thus these simulations are used to estimate the minimal cassette thickness required to handle a certain Lorentz force.

In Figure 4.16 the longitudinal stress in the winding pack is plotted as a function of cassette thickness. The cool-down results in a longitudinal compression of around $-14 \mathrm{MPa}$, virtually independent of the thickness of the aluminium cassette.

Upon applying an electromagnetic body force to the winding packs equal to that of normal operation, the longitudinal stress increases to around $-4 \mathrm{MPa}$. A further increase of the force level results in an increase of the stress that depends on the thickness of the cassette. $\mathrm{NbTi} / \mathrm{Cu}$ conductor can handle a tensile stress of $500 \mathrm{MPa}$ [39]. With an estimate that the uninsulated conductor occupies $68 \%$ of the winding pack volume, a maximum still reasonable winding pack longitudinal stress of $340 \mathrm{MPa}$ is found. Only at force levels of upwards of 7 times that in the demonstrator, in combination with a thin cassette, is this value approached. However, such a low cassette thickness does not provide enough pre-compression to prevent positive transverse stresses, as explained in the context of Figure 4.15. The minimum thickness corresponding to a certain Lorentz force level is indicated with a dashed black line in Figure 4.16.

Thus, it was concluded that the longitudinal stress component is not a major factor in determining the minimum winding pack thickness for NbTi MDS mag- 


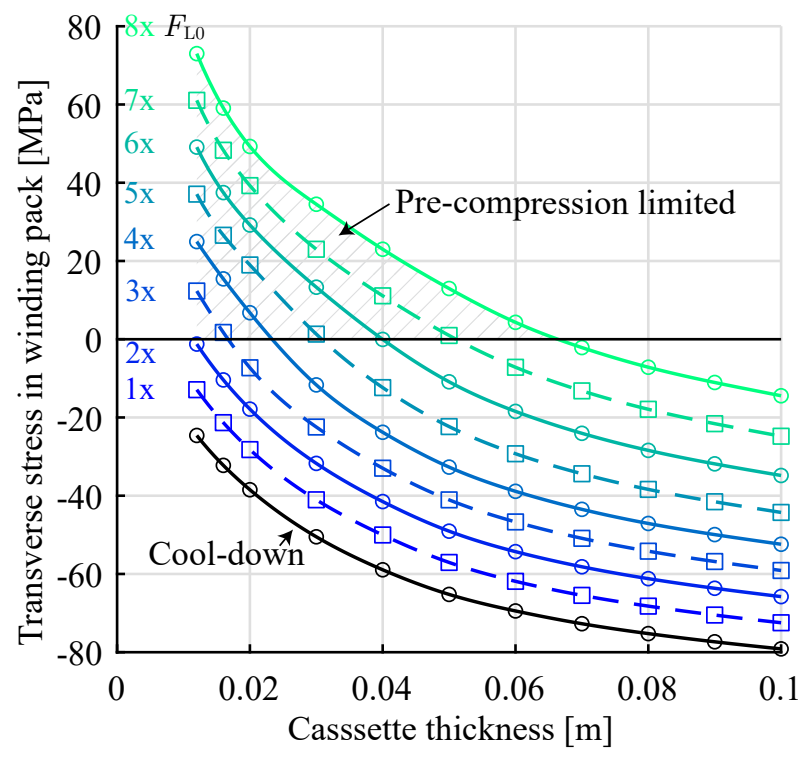

Figure 4.15: Transverse stress (in $x^{\prime} y$-plane) in the winding pack as a function of cassette thickness $d_{\text {cas }}$, see Figure 4.14, plotted for different Lorentz force levels, indicated by different colours. These levels are relative to the NbTi demonstrator $\left(F_{\mathrm{L} 0}\right)$. Data-points are indicated with markers. The hatched area indicates loadcases in which the winding pack in under positive (tensile) transverse stress.

nets. This conclusion may be different other superconductors. For example, for $\mathrm{Nb}_{3} \mathrm{Sn}$, with a maximum allowable longitudinal stress of $250 \mathrm{MPa}$ [39], a maximum longitudinal stress averaged over the winding pack of $188 \mathrm{MPa}$ is allowed. This value is exceeded for high Lorentz force levels in combination with a thin cassette $^{6}$

If the cassette is relatively thin, the winding pack dominates the thermal shrinkage of the cold mass, and if it is thick, the cassette dominates. Thus during cooldown a tensile stress develops in the cassette that decreases with increasing cassette thickness.

The von Mises stress in the aluminium is shown in Figure 4.17. This stress is estimated by multiplying the stress in the solid bulk (Figure 4.14) by a factor three to obtain the stress concentration around the holes, see Figure 4.9, that allow the room-temperature columns to pass through. The assumption is made that this factor three between the stress in the bulk of the aluminium and the stress

\footnotetext{
${ }^{6}$ Here we assume that the mechanical properties of $\mathrm{a} \mathrm{Nb}_{3} \mathrm{Sn}$-based winding pack are similar to that of the NbTi demonstrator.
} 


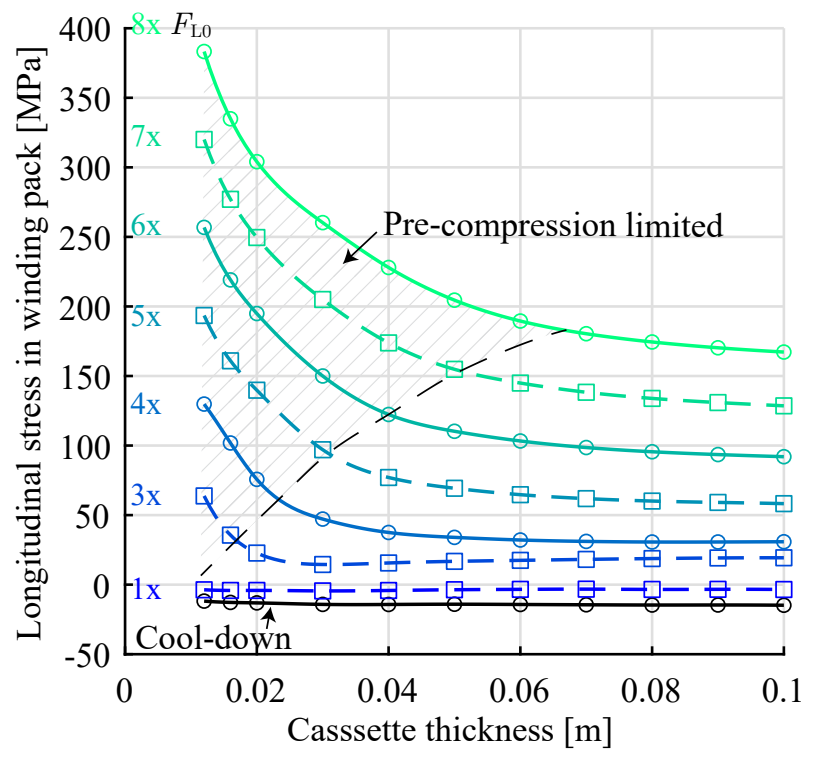

Figure 4.16: Longitudinal stress in the winding pack as a function of cassette thickness $d_{\text {cas }}$, see Figure 4.14, plotted for different force levels, indicated with different colours. Data-points are indicated with markers. The hatched area above the dashed line indicates load-cases in which the winding pack in under positive (tensile) transverse stress.

concentration around the holes is independent of the thickness of the cassette.

The minimum thickness that provides sufficient transverse pre-compression corresponding to a certain Lorentz force level is indicated with a dashed black line in Figure 4.17. The maximum stress is about the same as for the demonstrator. Higher stress levels may also be allowed as far as the aluminium is concerned, since aluminium alloys exist with a yield strength of at least $700 \mathrm{MPa}$ at cryogenic temperatures, for example 7175-T4 with $745 \mathrm{MPa}$ and 7075-T6 with $750 \mathrm{MPa}$ [73].

In conclusion, the stress in the cassette is not a limiting factor in the performance (i.e. the distance between the coils and the ferrofluid). Thus, removing the holes that allow the room-temperature columns would not bring any advantages as far a the required cassette thickness is concerned. The columns are therefore a good solution for not just the demonstrator but also for more powerful MDS magnets.

A second conclusion is that the main factor determining the minimum cassette thickness is the amount of pre-compression applied by the cassette due to 


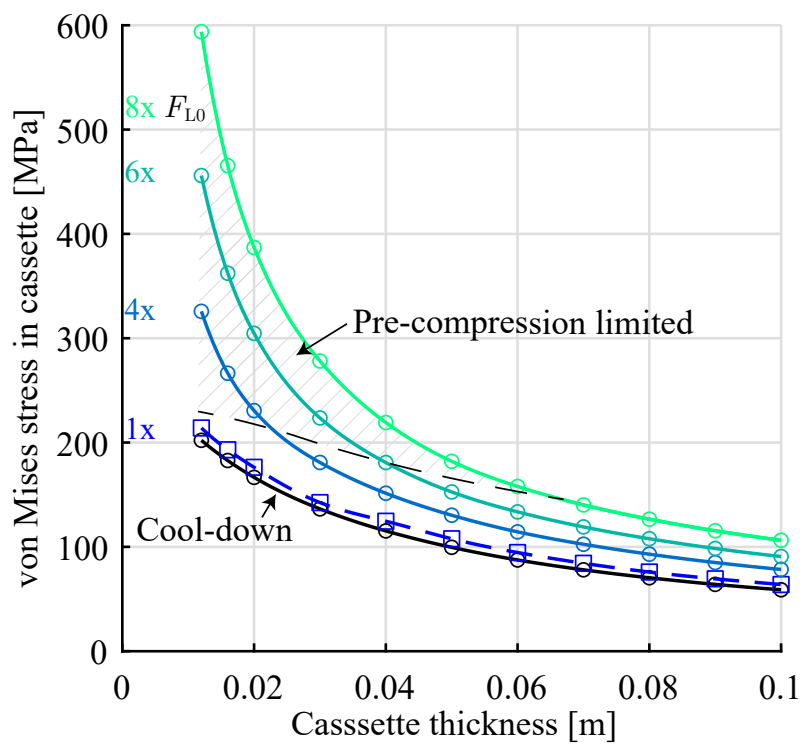

Figure 4.17: Von Mises stress in the aluminium alloy cassette as a function of cassette thickness $d_{\text {cas }}$, see Figure 4.14, plotted for different force levels. Datapoints are indicated with markers. The hatched area indicates load-cases in which the winding pack in under positive (tensile) transverse stress.

differential thermal shrinkage during cool-down.

Another conclusion is that very high strength aluminium alloy is not required.

As a thicker cassette implies a larger distance between the coils and the ferrofluid, it is of interest to consider designs that do not require the presence of a strong cassette on the fluid side of the magnet. Several such concepts are presented in the next section.

\subsubsection{Asymmetric cassette design attempts}

In this section a few earlier concepts for the mechanical design are presented, which were explored but found unsuitable. Three concepts were conceived aiming to handle the Lorentz forces by using the space available below the coils, thus reducing the thickness of the cassette at the top-side and hence also the fluidmagnet distance. These are schematically depicted in Figure 4.18a-c. A fourth concept was to enclose the coils in a steel reinforcement ring, see Figure 4.18d.

The first, most simple approach taken was to increase the thickness of the mechanical cassette only of the bottom, as shown in Figure 4.18a. However, this 


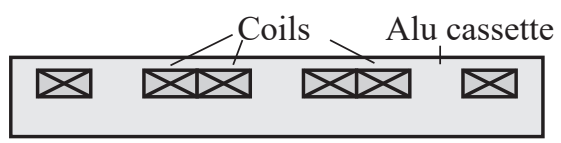

a) Increased thickness of bottom plate

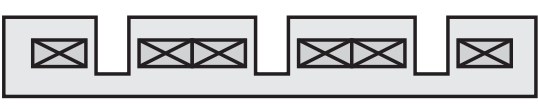

b) Room for ribs in cryostat top plate
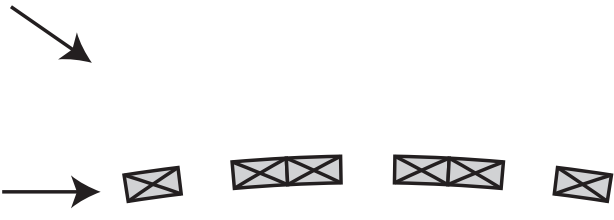

Vertical bending

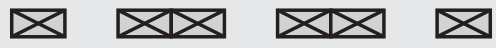

c) Reinforcement plate or

beams on bottom plate
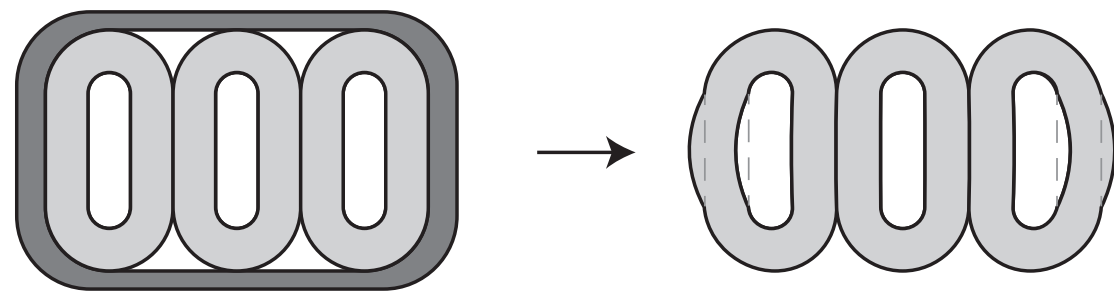

Horizontal bending

Figure 4.18: Compilation of concepts to reduce the coil-to-fluid distance with no satisfactory results. a) Increasing the thickness of the bottom part of the cassette results in vertical bending of the coils, due to the asymmetric contraction during cool-down. b) Cutting out part of the top of the cassette enables the use of a ribbed, thus stronger, cryostat wall, but also results in too much bending. c) Reinforcing the cassette on the bottom by using stiff beams also is not useful. $d$ ) Making use of the space on the sides of the coils by using a reinforcement ring leads to horizontal bending, since the ring is not stiff enough due to the long arm over which the Lorentz force has to be transferred.

caused a significant bending of the winding pack, since the thermal contraction of the bottom plate exerts a stronger force than the top plate, resulting in asymmetric tension on the winding pack.

The second option, illustrated in Figure 4.18b, utilizes slits between the coils, to allow for reinforcement ribs on the cryostat top plate. Similar bending as in the previous design is found, as the asymmetric contraction is still present.

In an attempt to neutralize the effect of the asymmetric contraction, a material 
with a low thermal contraction coefficient could be attached to the bottom of the cassette, see Figure 4.18c. Ti-6Al-4V has a thermal contraction of only $0.173 \%$ between room temperature and $4.2 \mathrm{~K}$, but a relatively average Young's modulus of $114 \mathrm{GPa}$ and it is prone to brittle fracture at high stress levels [42]. A different material option could be Hastelloy C-276, with a thermal contraction of $0.218 \%$ and Young's modulus of $205 \mathrm{GPa}$ [42]. Alternatively, one may even consider a ferromagnetic material, such as $\mathrm{FeNi}$, with $0.195 \%$ contraction for $\mathrm{Fe}-9 \% \mathrm{Ni}$ [42]. An additional benefit would be a reduced stray magnetic field around the magnet (the $0.5 \mathrm{mT}$ line is at around $3.5 \mathrm{~m}$ from the center of the demonstrator).

Simply using a flat plate bolted to the aluminium alloy base plate has the downside that the thermal contraction force is not equal in the two horizontal directions. Thus the coils still see an asymmetric force causing them to bend. One would need to resort to a ribbed structure to equalize the thermal contraction in both horizontal directions.

A further idea was to enclose the coils in a reinforcement ring, see Figure 4.18d. At first sight, this symmetric approach looks promising since no vertical bending of the coils is present. However, even a $0.20 \mathrm{~m}$ thick steel ring was found to lack the required stiffness to prevent horizontal bending. The effective moment caused by the Lorentz force in the $x^{\prime}$-direction is large as a result of the long arm. Moreover, this type of mechanical enclosure results in a high tensile stress of some $300 \mathrm{MPa}$ in the coil heads.

From the four options only the third one (Figure 4.18c) shows some potential for further exploration. The gain of in magnetic field gradient would be about $13 \%$ for the demonstrator. This is the effect of subtracting the cassette top plate thickness of $12 \mathrm{~mm}$ from the coil-to-fluid distance. The loss in performance due to the symmetric design is considered acceptable in view of the higher simplicity of the mechanical structure for the demonstrator magnet. As detailed in Section 3.2, however, the performance of high-field MDS magnets can be significantly improved if a suitable asymmetric mechanical design can be found.

The idea of placing magnetic iron underneath the coils to help boost the magnitude of the magnetic field was discarded early on in the design process in order to have more freedom in the design to meet the mechanical- and thermal requirements of the magnet. A "back-iron" underneath the coils would take up space that is now available for the cassette, high-purity thermal bus, instrumentation, and assembly access. Also, the added weight of the iron was considered unattractive as it would require increasing the strength of the warm-cold support structure and hence an increased heat in-leak. Thus iron was excluded from the design essentially for simplicity. 


\subsection{Cold mass support structure}

THIS section concerns the design of the G11 structure that hold the MDS cold 1 mass in place. Since these pillars are at room temperature on one side and at cryogenic temperature at the other, they will be referred to as warm-cold pillars.

The warm-cold pillars are shown in Figure 4.19 and are made up out of four hollow tubes ( $z^{\prime}$ oriented and indicated as "vertical"), and four solid tie-rods (indicated as "horizontal"). A schematic is shown in Figure 4.19. Two of these tie-rods are placed in the $x^{\prime}$-direction with a $+3^{\circ}$ angle offset in the $x^{\prime} z^{\prime}$-plane, and two at $-3^{\circ}$. We will assume that they are actually oriented in the $x^{\prime}$-direction, neglecting the $3^{\circ}$ offset.

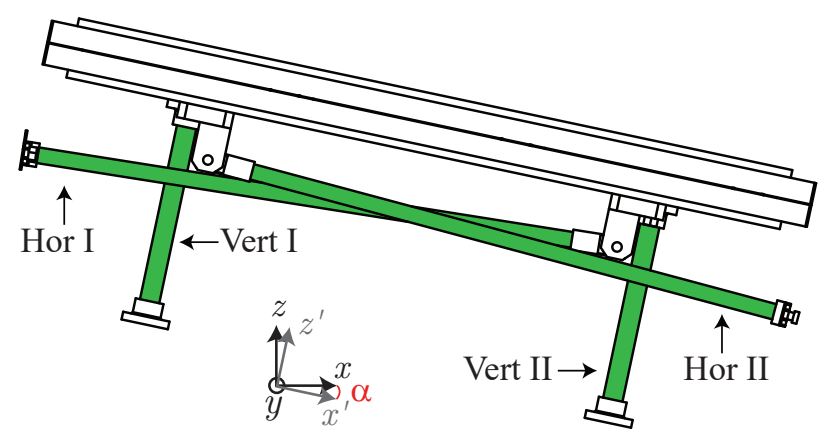

Figure 4.19: Side view of the cassette that encloses the coils and of the pillar structure suspending it inside the cryostat. The structure consists of four solid horizontal tie-rods (two "Hor I" and two "Hor II") and four vertical hollow pillars ("Vert I" and "Vert II"). They are made from G11 fibreglass tube and the tube ends are glued in stainless steel sockets.

In the design of the pillar structure, a trade-off is made between heat in-leak, requiring a small cross-sectional area of the pillars, and strength against buckling, requiring a certain minimum cross-section. A safety factor of four was chosen to determine the tube size and wall dimensions. Furthermore, upon energizing, the pillars may—depending on the position and scenario—also become loaded in tension.

First the critical buckling load is considered and next the heat in-leak through the pillars. Then, a figure-of-merit is introduced to compare materials to find the best pillar material optimized for high buckling strength and low heat in-leak. It was found that G11 is the optimum practical material. Additionally, the pillar design is checked for tensile strength, since it is required to handle the attraction force between ferrofluid and coils. These analytical calculations are compared to 
FEM simulation results.

Next the resonance frequencies of the cold mass and the selected pillar structure are considered. These allow to check the dynamic behaviour of the system in the case of external sources like seismic events.

Lastly, reducing the effect of external vibrations by means of a vibration isolator is presented.

\subsubsection{Buckling: critical load}

Euler's buckling criterion for a tube determines that buckling will occur once the compressive load exceeds a certain critical value $F_{\text {cr }}[42]$ :

$$
F_{\mathrm{cr}}=\frac{C \pi^{3} E}{L^{2}} \frac{\left(D_{\mathrm{o}}^{4}-D_{\mathrm{i}}^{4}\right)}{64},
$$

where $E$ is Young's modulus, $L$ is the length of the pillar, and $D_{\mathrm{o}}$ and $D_{\mathrm{i}}$ its outer- and inner diameters, respectively. The constant $C$ in the buckling criterion depends on the boundary conditions of the loading. A worst-case value of 0.25 is assumed [42].

The critical load can be seen as the gravitational load $F_{z}$ on each pillar, times a safety factor of $N$ :

$$
F_{\text {cr }}=\frac{N F_{z}}{n}
$$

Here $n$ is the number of pillars and the $12^{\circ}$ angle between the $z^{\prime}$ - and $z$ axis is neglected.

The heat in-leak to the cold end of $n$ tubes is given by

$$
\dot{Q}_{\mathrm{c}, \mathrm{total}}=\frac{n \bar{k} \Delta T}{L_{2}} \frac{\pi}{4}\left(D_{\mathrm{o}}^{2}-D_{\mathrm{i}}^{2}\right)
$$

where $L_{2}$ is the distance between the cold end of the pillar and the heat sink, as illustrated in Figure 4.20. The heat sink temperature is set to $77 \mathrm{~K}$ in the calculations. $\bar{k}$ is the effective heat conductivity coefficient, determined by a thermal conductivity integral, for the temperature gradient across this section of the pillar $(\Delta T=77 \mathrm{~K}-4 \mathrm{~K})$.

The total heat in-leak of $n$ pillars that can withstand buckling, including the safety factor $N$, is obtained by solving Eq. 4.4 for $D_{\mathrm{o}}$ and filling this expression 


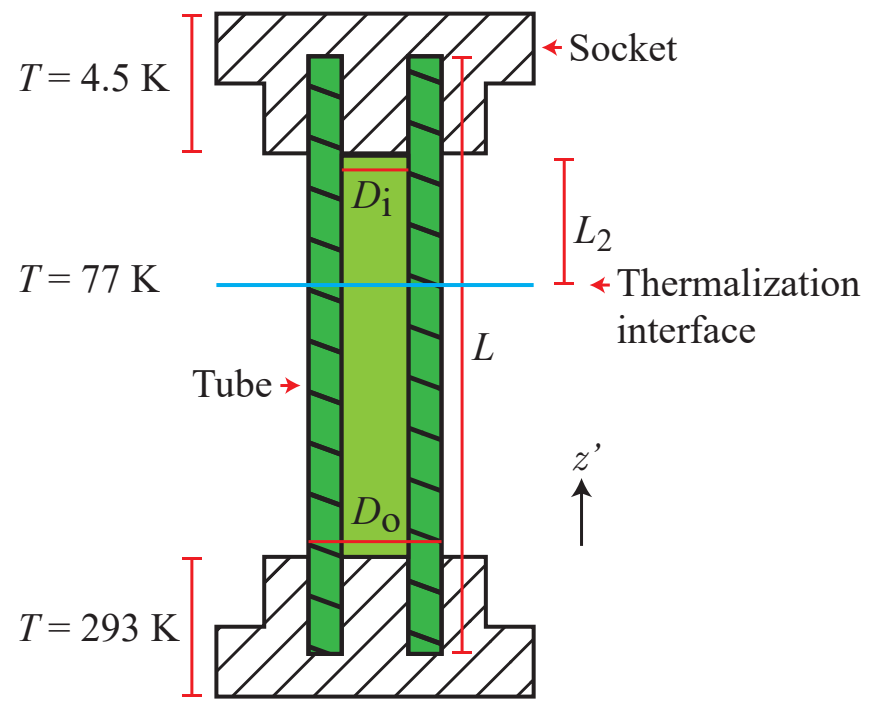

Figure 4.20: Cross-section of a G11 hollow pillar with length L, inner diameter $D_{\mathrm{i}}$ and outer diameter $D_{\mathrm{o}}$, as well as two sockets. The aim of the pillar is to separate the cold mass from the cryostat bottom plate with minimal heat in-leak. The fixtures, made from stainless steel 304, are considered to be at a uniform temperature, since their thermal conductivity is around 20 times higher than that of G11 [42] both at $4.5 \mathrm{~K}$ and at room temperature. The tube is thermalized at an intermediate temperature of around $77 \mathrm{~K}$ by a connection to the thermal shield, at a distance $L_{2}$ from the cold end 5.2.

in Eq. 4.6:

$$
\dot{Q}_{\text {c,total }}=\frac{n \bar{k} A \Delta T}{L_{2}} \frac{\pi}{4}\left[\sqrt{\left(\frac{64 N L^{2} F_{z}}{n C \pi^{3} E}+D_{\mathrm{i}}^{4}\right)}-D_{\mathrm{i}}^{2}\right] .
$$

In the case of a solid rod $\left(D_{\mathrm{i}}=0\right)$, this reduces to

$$
\dot{Q}_{\mathrm{c}, \text { total }}=2 \bar{k} \Delta T \frac{L}{L_{2}} \sqrt{\left(\frac{N n F_{z}}{C \pi E}\right)} .
$$

A figure of merit (FOM) is defined to compare materials to be used for the pillars. Eq. 4.7 and Eq. 4.8 indicate that the heat in-leak is minimized by minimizing 
$\bar{k} / \sqrt{E}$. Therefore,

$$
F O M \equiv \frac{\bar{k}}{\sqrt{E}} .
$$

Note that the length of the pillar does not influence the heat in-leak at $4.5 \mathrm{~K}$, only the relative placement of the radiation shield $L / L_{2}$ is important ${ }^{7}$. With the number of pillars the heat in-leak increases as well. Hence it is more optimal to use a few relatively thick pillars than many thin ones.

Between $4.5 \mathrm{~K}$ and $77 \mathrm{~K}$, the effective thermal conductivity is $0.21 \mathrm{~W} / \mathrm{m} / \mathrm{K}$ for G11 and $4.5 \mathrm{~W} / \mathrm{m} / \mathrm{K}$ for SS304 stainless steel, calculated using thermal conductivity integrals [42]. Young's moduli at room temperature are $20 \mathrm{GPa}$ for G11 and $195 \mathrm{GPa}$ for SS304 steel, respectively [42, 76]. This gives

$$
\begin{aligned}
F O M(\mathrm{G} 11) & =\frac{0.21}{\sqrt{20}} \approx 0.047, \\
F O M(\mathrm{SS} 304) & =\frac{4.5}{\sqrt{195}} \approx 0.32 .
\end{aligned}
$$

G11 thus allows to reduce the heat in-leak by a factor 7 compared to SS304 when using rods. The next section concerns the attainable reduction in heat in-leak by using hollow tubes.

\subsubsection{Optimized pillar dimensions against buckling}

Eq. 4.7 is used to relate the diameter of the warm-cold pillars to the heat in-leak for a gravitational load of $5 \mathrm{kN}$. A conservative safety factor of four is included. The result is shown in Figure 4.21, for a 4-pillar G11 design, and in Figure 4.22 for four SS304 pillars. The heat load is summed for all four pillars. The thermal heat sink is connected at $L_{2}=0.37 L$ (Section 5.2). The length of the pillars is set to $0.272 \mathrm{~m}$. Even though increasing $L_{2}$ decreases the in-leak at the cold side, in this case the need for accessibility during assembly did not allow a larger distance between heat sink and cassette.

The solid line shows how the outer diameter of the pillar needs to increase to reduce the heat load. The inner diameter is shown by the dashed line. The pillar quickly becomes thin-walled as the diameter increases, i.e. the cross-sectional area decreases and thus the heat in-leak is reduced. However, the reduced crosssectional area also implies an increase of the experienced stress following fluid-

\footnotetext{
${ }^{7}$ The distance between the room-temperature socket and the heat-sink section does of course influence the heat in-leak at the sink, and therefore its temperature due to the limited cooling power of the cooler. Consequently, it has an impact on the heat-load at $4.5 \mathrm{~K}$. This effect is not taken into account here.
} 


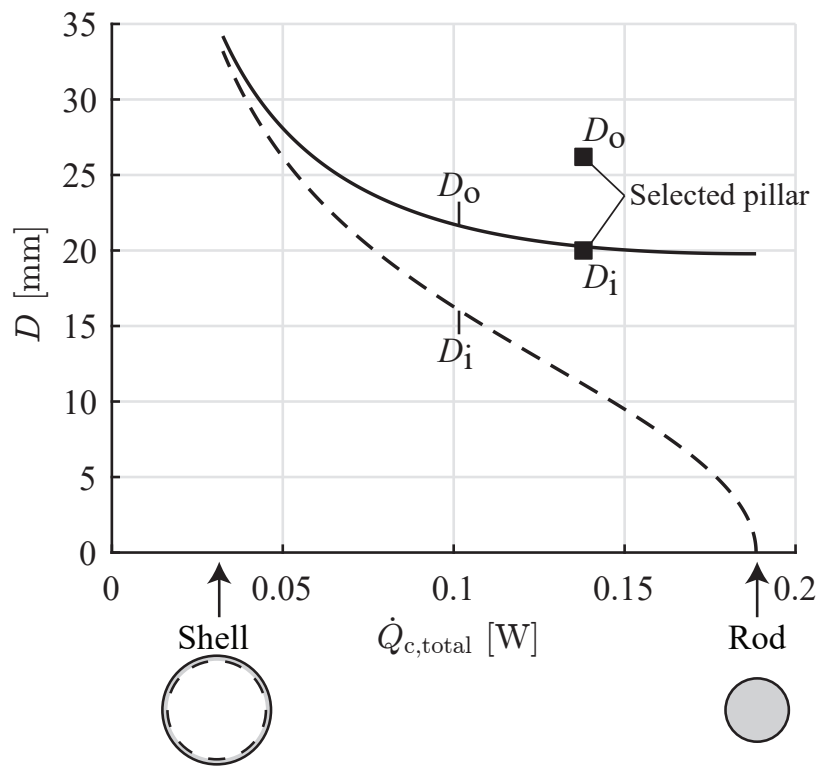

Figure 4.21: Relation between the inner- and outer diameters of the pillars and the heat load through four pillars at $4.5 \mathrm{~K}, \dot{Q}_{\mathrm{c}, \text { total }}$, for a four-pillar 111 configuration with $L_{2}=0.37 \mathrm{~L}=100 \mathrm{~mm}$, see Figure 4.20. The heat load and diameters are linked through the required resistance against buckling. The selected pillars have an inner diameter of $20 \mathrm{~mm}$ and an outside diameter of $26.2 \mathrm{~mm}$ and are indicated by black square markers. It can be seen that these pillars are somewhat over-dimensioned.

cold mass attraction. For SS304 this limits the minimal heat load to $0.7 \mathrm{~W}$, since for smaller cross-sectional areas the tensile stress becomes unacceptable.

G11 is in the buckling-limited region for all plotted diameters. As a safety margin, half the room temperature yield stress is the maximum. This yield stress is $240 \mathrm{MPa}$ for SS304 and $375 \mathrm{MPa}$ for G11 [42].

Even with four G11 solid rods, the total heat in-leak is only $0.19 \mathrm{~W}$. Thus G11 is chosen as the material for the warm-cold pillars. Very thin-walled tubes theoretically allow for a low heat load; however, any imperfections in wall thickness or tube wall imperfections will then have a large effect on the buckling strength.

The calculations in the preceding section are based on vertical pillars with a cross-sectional profile that is optimized for the buckling resistance versus the heat in-leak via conduction. However, it was estimated that the thermal budget, as discussed in Section 5.2, allows for vertical pillars with a larger cross-sectional area. So the chosen pillars have inner and outer diameters of $20 \mathrm{~mm}$ and $26.2 \mathrm{~mm}$, re- 


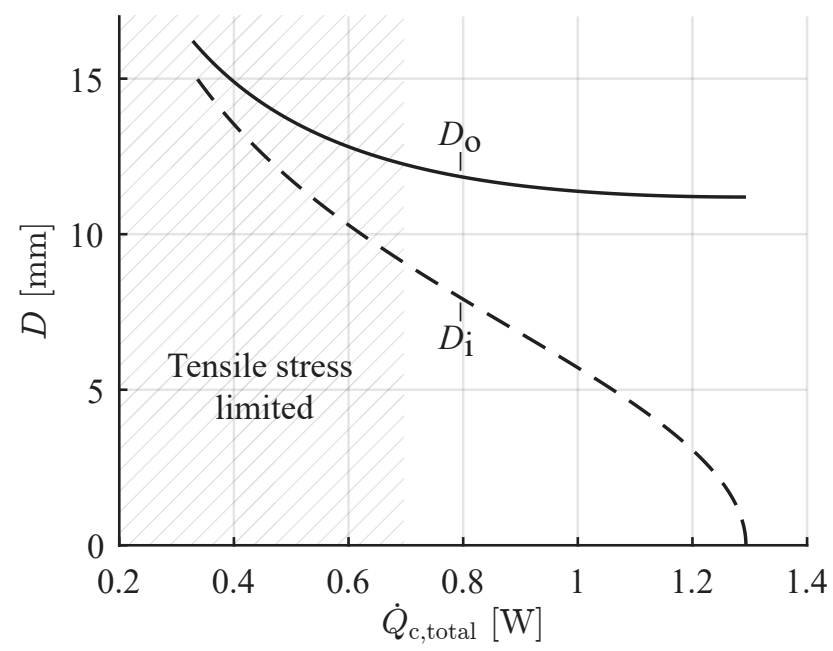

Figure 4.22: Relation between the inner-and outer diameter of the pillars and the heat load through four pillars at $4 \mathrm{~K}, \dot{Q}_{\mathrm{c}, \text { total, }}$, for a four-pillar SS304 configuration with $L_{2}=0.37$ L, see Figure 4.20 .

spectively. The main motivation behind this choice is that a mechanical failure has much worse effects than a slightly higher operating temperature. If a pillar breaks, the magnet stops being useful, whereas at a slightly higher operating temperature one can still utilize the magnet with a smaller temperature margin or with a lower magnetic field. The selected pillars have a buckling strength of $10 \mathrm{kN}$, while the gravitational load is $1.3 \mathrm{kN}$. Thus a safety factor of 8 is present.

The four vertical warm-cold pillars of the demonstrator magnet introduce a heat load at $4.5 \mathrm{~K}$ of $138 \mathrm{~mW}$, still five times lower than what can be achieved with stainless steel pillars.

It was decided that further methods to increase the buckling resistance were not necessary, since with the chosen pillar structure the heat load is low enough. For example, buckling strength can also be substantially improved by reducing the effective length $L$ of the pillars by interconnecting them at 1 or 2 temperature levels.

The horizontal tie rods, shown in Figure 4.19, do not experience a strong compressive load and are not in risk of buckling. The heat in-leak of four of these rods, thermalized to $77 \mathrm{~K}$ at half a meter from the cold mass, and with a $20 \mathrm{~mm}$ diameter, is estimated at $39 \mathrm{~mW}$. This diameter is chosen to provide sufficient stiffness. Both the rods and tubes are manufactured by Von Roll, and the specific type of G11 is Vetronite [76]. The stresses in the pillars structure in various loadcases are the focus of the next section. 


\subsubsection{Vertical \& horizontal pillar structures}

Two methods are used to estimate the stress in the pillars and tie rods. The first is analytical and based on the assumption that the vertical pillars handle all forces in the $z^{\prime}$-direction and the tie rods all $x^{\prime}$-forces:

$$
\begin{aligned}
F_{\text {vert-pillars }} & =F_{z} \cos \alpha+F_{\text {mag }, z^{\prime}} \pm F_{\text {torque }} ; \\
F_{\text {hor-pillars }} & =F_{z} \sin \alpha+F_{\text {mag }, x^{\prime}} .
\end{aligned}
$$

The torque on the tilted cassette due to the force between coils and ferrofluid only causes stress in the vertical pillars, as the tie rods are assumed to be parallel to the cassette.

During pumping of the cryostat, the cryostat wall at the warm end of the horizontal tie rods moves inwards $0.35 \mathrm{~mm}$ (see Section 4.2). This results in a compressive force of $3 \mathrm{kN}$ per rod, $2 / 3$ of the buckling resistance.

When the cold mass is cooled down to $4.5 \mathrm{~K}$, the vertical pillars shrink and the cold mass is free to move down due to the low stiffness of the tie rods in their transverse direction. Thus no thermal stress is created in the bulk of the vertical pillars. The connection of the pillars to the fixture on the cold side was evaluated in Section 4.4.5. The tie rods do develop a thermal stress and this will be estimated next.

The cassette itself is considered to be a rigid object that shrinks by $0.37 \%$. This corresponds to the shrinkage of the cold mass in the $x^{\prime}$-direction as calculated in Section 4.4.5. The assumption of a rigid object can be made as the pillars have a low stiffness compared to the cassette.

The horizontal rods are thermalized roughly half a meter from the cassette and have a total length of $0.72 \mathrm{~m}$. The fibre direction is longitudinal. To estimate the thermal contraction of these tie rods, a simple 1D FEM calculation is performed by which the rod is divided into a section of $0.22 \mathrm{~m}$ with one end at $293 \mathrm{~K}$ and one end at $77 \mathrm{~K}$, and a $0.5 \mathrm{~m}$ section with one end at $77 \mathrm{~K}$ and the other at $4.5 \mathrm{~K}$. Thermal contraction and conductivity are taken from [42]. The result is that the tie rods shrinks by $0.194 \%$.

Part of the contraction will not result in stress, since the cold end of the rod moves over a distance $\Delta x^{\prime}=-0.0037 \cdot 0.30 \mathrm{~m}=-1.1 \mathrm{~mm}$ due to the shrinking cassette. The resulting thermal stress in the tie rods is

$$
\begin{aligned}
\sigma_{\text {thermal }, x^{\prime}} & =\varepsilon E=\frac{\Delta L}{L} E \\
& =(0.00194-0.0037 \cdot 0.30 \mathrm{~m} / 0.72 \mathrm{~m}) \cdot 2 \cdot 10^{10} \mathrm{~Pa} \\
& =7.8 \mathrm{MPa} .
\end{aligned}
$$


The stress in the pillars during different loading scenarios are presented in Table 4.6. The magnitudes of the forces between ferrofluid and coils are presented in Sections 3.2 and 3.3, respectively. The two analysed scenarios are those in which the total force and in which the exerted torque are maximum. The first scenario corresponds to normal operation in which the magnet is fully energized and attracts the entire fluid volume, the second one to a situation where part of the fluid is accidentally drained and a highly asymmetric force balance occurs. The attraction force in both scenarios has been multiplied by a factor 3.3 to represent an unstable ferrofluid (Section 3.1.5). Data from a COMSOL model agree well with analytical estimates. During all load-cases the stress is well below the allowed values. The vertical pillars are under compression unless the magnet is energized and there is a ferrofluid present, see Figure 3.1. At this point, it depends on the filling level of the fluid tank whether the Vert I pillars see a compressiveor a tensile force.

The mentioned simulation model has a geometry, see Figure 4.23, representing the coils and pillars, as well as a 1-piece cassette-yoke-copper composite. The total mass of the combined object is $520 \mathrm{~kg}^{8}$.

Table 4.6: Average vertical stress $\bar{\sigma}_{z^{\prime}}$ in vertical warm-cold pillars and average horizontal stress $\bar{\sigma}_{x^{\prime}}$ in horizontal warm-cold pillars for various load cases, calculated both using analytical expressions and a simplified 3D COMSOL model, respectively. FEM results are presented in brackets.

\begin{tabular}{l|ll|ll} 
& \multicolumn{2}{|l}{ Stress $\bar{\sigma}_{z^{\prime}}[\mathrm{MPa}]$} & \multicolumn{2}{l}{ Stress $\bar{\sigma}_{x^{\prime}}[\mathrm{MPa}]$} \\
Case & Vert I & Vert II & Hor I & Hor II \\
\hline Room temperature & $-6(-5)$ & $-6(-6)$ & $-9(-9)$ & $-11(-11)$ \\
Cool-down & $-6(-4)$ & $-6(-5)$ & $-1(0)$ & $-3(-2)$ \\
High torque & $-5(-4)$ & $+17(+17)$ & $+4(+2)$ & $-7(-5)$ \\
Full fluid bed & $+19(+19)$ & $+24(+24)$ & $0(-2)$ & $-5(-5)$
\end{tabular}

When comparing the vertical displacement of the cold mass after cool-down $(-0.5 \mathrm{~mm})$ and upon energizing, it is found that the cold mass moves up by $0.37 \mathrm{~mm}$ due to the ferrofluid-to-magnet attraction, in the case of a full fluid bed.

\footnotetext{
${ }^{8}$ The mass in a more detailed design changed to $550 \mathrm{~kg}$.
} 


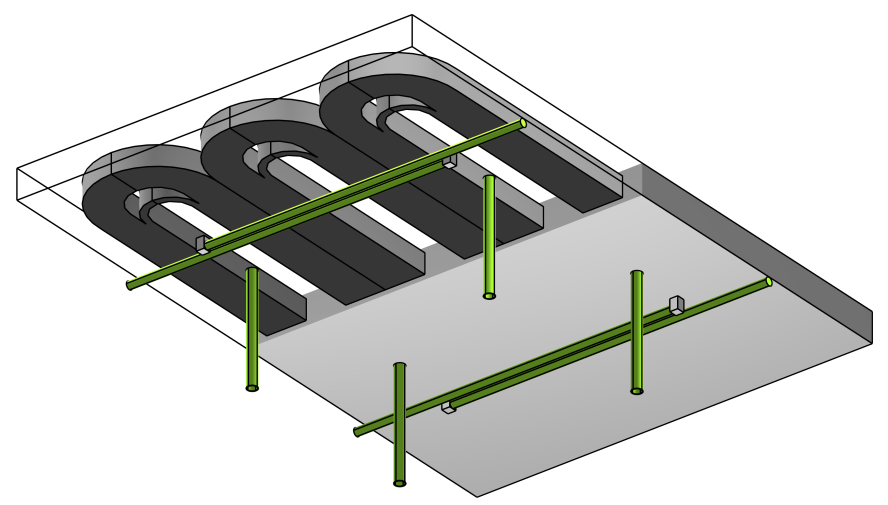

Figure 4.23: Simplified geometry used in simulation of the pillar-cold mass structure. The mechanical cassette is modelled as a single object with mixed properties of the aluminium alloy, stainless steel yoke, and copper pieces making up the actual cassette. Half of this object is shown in the figure.

\subsubsection{Resonance frequencies of cassette-pillar structure}

With MDS being an industrial process, a magnet suitable for this kind of environment may be subjected to external vibrations and shocks, including seismic, to an extent which a laboratory demonstrator may not be.

The building in which the demonstrator magnet was originally intended to be installed at University of Delft also houses a testing facility for concrete beams. These beams are destructively tested, generating vibrations that, if significant enough, could damage the magnet. Instead of trying to find out if this particular magnet will operate stably in this particular building, a more general method is followed that can be applied to future superconducting MDS magnets. The result is that the demonstrator system has to be installed another building.

First an analytical estimate is made of the resonance frequencies, which is later compared to COMSOL simulation results. The advantage of including a COMSOL simulation is that the significance of stress-stiffening on the resonance frequencies can be investigated. This stress-stiffening is a result of the tensile load that develops on the tie rods during cool-down, and on the vertical pillars due to the magnet-fluid force, as discussed in the previous section.

In the analytical calculation, the warm ends of the pillars are fixed, as the cryostat body is considered to be rigid. The configuration is represented as a simple lumped mechanical system with the cold mass mass $m$ connected via the warm-cold pillars, represented by a spring $k$, to the rigid and static cryostat body.

Let us assume that there are three main eigenfrequencies, one for each dir- 
ection. The stiffness of the spring in de $x^{\prime}$-direction is dominated by the four horizontal $x$-pillars, and in the $z^{\prime}$-direction by the vertical pillars. The stiffnesses, reported in Table 4.7, are estimated by

$$
k_{x^{\prime}}=n_{x^{\prime}} \frac{E A_{x^{\prime}}}{L_{x^{\prime}}}, \quad \quad k_{z^{\prime}}=n_{z^{\prime}} \frac{E A_{z^{\prime}}}{L_{z^{\prime}}},
$$

where $A_{x^{\prime}}$ and $A_{z^{\prime}}$ are the surface areas of the horizontal and vertical pillars, respectively, $L_{x^{\prime}}$ and $L_{z^{\prime}}$ their length, and where $n$ is the number of pillars [80]. As for the stiffness in the $y$-direction, we consider both the vertical pillars and tie rods as beams being loaded perpendicular to their longitudinal axis. This leads to a stiffness

$$
k_{y}=\frac{3 n_{z^{\prime}} E I_{z^{\prime}}}{L_{z^{\prime}}^{3}}+\frac{3 n_{x^{\prime}} E I_{x^{\prime}}}{L_{x^{\prime}}^{3}}=\frac{3 \pi n_{z^{\prime}} E\left(D_{\mathrm{o}, z^{\prime}}^{4}-D_{\mathrm{i}, z^{\prime}}^{4}\right)}{64 L_{z^{\prime}}^{3}}+\frac{3 \pi n_{x^{\prime}} E D_{\mathrm{o}, x^{\prime}}^{4}}{64 L_{x^{\prime}}^{3}} .
$$

The tie rods only contribute $2 \%$ to the stiffness in the $y$-direction, due to the $L^{3}$ term favouring short pillar lengths. From the calculated stiffnesses the resonance frequencies are calculated using

$$
f=\frac{1}{2 \pi} \sqrt{\frac{k}{m}}
$$

where the mass $m$ of the cold mass is set to $520 \mathrm{~kg}$. The results can be found in Table 4.7 as well. As expected, the resonance frequency in the $y$-direction is much lower than in the other two directions, as the stiffness in $y$-direction is two orders of magnitude lower than in the other two directions.

Table 4.7: Analytical estimate of stiffness of pillar structure (Eq. 4.14,4.15) and resonance frequencies $f$ (Eq. 4.16 and COMSOL). Estimated for a non-prestressed system.

\begin{tabular}{llll} 
Direction & $\begin{array}{l}\text { Stiffness } k \\
{[\mathrm{~N} / \mathrm{m}]}\end{array}$ & $\begin{array}{l}f \text { (Eq. 4.16) } \\
{[\mathrm{Hz}]}\end{array}$ & $\begin{array}{l}f \text { (COMSOL) } \\
{[\mathrm{Hz}]}\end{array}$ \\
\hline$x^{\prime}$ & $3.1 \cdot 10^{7}$ & 39 & 36 \\
$y$ & $7.4 \cdot 10^{5}$ & 6.0 & 5.8 \\
$z^{\prime}$ & $6.6 \cdot 10^{7}$ & 57 & 53
\end{tabular}

To verify the results from this simple estimate an eigenfrequency study is 
performed in COMSOL using the geometry shown in Figure 4.23. The results are shown in Table 4.7.

The resonance frequencies resulting from the COMSOL simulation correspond well with the analytical calculation. The presented eigenvalues shown are calculated for the scenario in which the cold mass is at room temperature. The other load cases provide no significant differences as only the lowest resonance frequency shows a shift from $5.8 \mathrm{~Hz}$ to $6.3 \mathrm{~Hz}$. This change is likely due to the stress stiffening effect caused by the tensile load on the tie rods on cool-down and by the tensile stress on the vertical pillars due to the fluid-cold mass attraction.

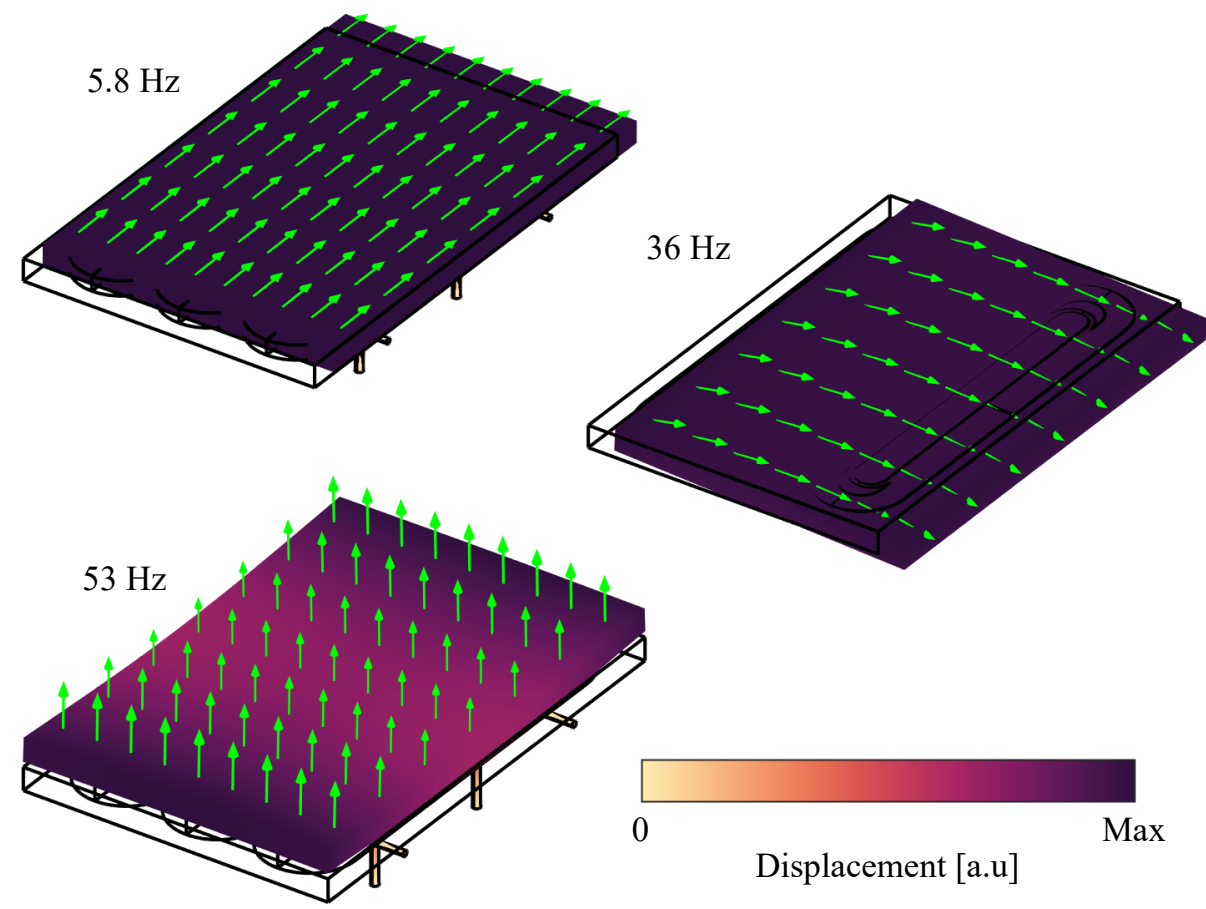

Figure 4.24: Displacement shapes of the four major resonance frequencies of the cold mass-pillar structure are shown. The cold mass vibrates as a rigid body in the $y$-direction at $5.8 \mathrm{~Hz}$, in the $x^{\prime}$-direction at $36 \mathrm{~Hz}$ and in the $z^{\prime}$-direction at $53 \mathrm{~Hz}$.

\subsubsection{Response of the cold mass to shocks}

To get an idea of the amplitude of the displacement and stress in the cold mass and the pillars due to external vibrations, a response spectrum analysis is performed, 
leaning heavily on design rules for earthquake resistant buildings [81]. The goal is to find the response of the cold mass to an accelerating ground plane. As the frequency spectrum of earthquakes is well studied, the possible vibrations due to destructive concrete testing will be modelled as a small earthquake. As a rough estimate of the effect is sought, this is deemed sufficient for a first approach.

The same lumped system is considered as in the previous section with the cryostat as a rigid body, moving at the same amplitude and phase as the ground. After that, a model including a vibration isolator between ground and cryostat is evaluated, to estimate the potential reduction of vibrations transmitted to the cold mass.

Figure 4.25 shows an example of the frequency band of possible earthquakes. The shape of the curve depends on the geographical location and the type of soil. The plotted spectrum is valid for a dense or medium-dense sand or clay soil [81]. The magnitude of the used curves corresponds to a 'weak', or scale III, earthquake on the Modified Mercalli Intensity scale ${ }^{9}$. The peak ground acceleration for a scale III earthquake is $0.08 \mathrm{~m} / \mathrm{s}$ on average [83].

Using the calculated eigenfrequencies and the earthquake spectrum as an input, the response of the cold mass is calculated using mode superposition. This method is able to calculate the approximate dynamic response of a structure by a superposition of its eigenmodes, weighing each eigenmode with its modal mass [84]. The calculated eigenfrequencies were found to contain $>99 \%$ of the relative modal mass in each direction. In response spectrum analysis, the load represents a homogeneous acceleration in each spatial direction. Thus, each eigenmode can be considered to have a fraction of the total mass associated with it. The sum over all modal mass components in each separate direction is equal to the mass of the structure. The stresses and displacements calculated by this method are all absolute valued.

The von Mises stress in the G11 pillars reaches $200 \mathrm{MPa}$ in this simulation, as indicated in Figure 4.26. This is close to the compressive- and tensile strength of this material, which are both $300 \mathrm{MPa}$ [76]. Also, the compressive load on the pillars could reach up to $26 \mathrm{kN}$ per pillar, more than their buckling strength without any safety factor of $10 \mathrm{kN}$. This buckling strength is calculated in Section 4.5.2.

The maximum displacement of the cold mass relative to the moving ground is shown in Figure 4.27. The displacement is mainly in the $y$-direction as the $5.8 \mathrm{~Hz}$ eigenfrequency is located in the high-acceleration regime of the input spectrum.

\footnotetext{
${ }^{9}$ Scale III on the Modified Mercalli Intensity scale: Felt quite noticeably by persons indoors, especially on upper floors of buildings. Many people do not recognize it as an earthquake. Standing motor cars may rock slightly. Vibrations similar to the passing of a truck [82].
} 


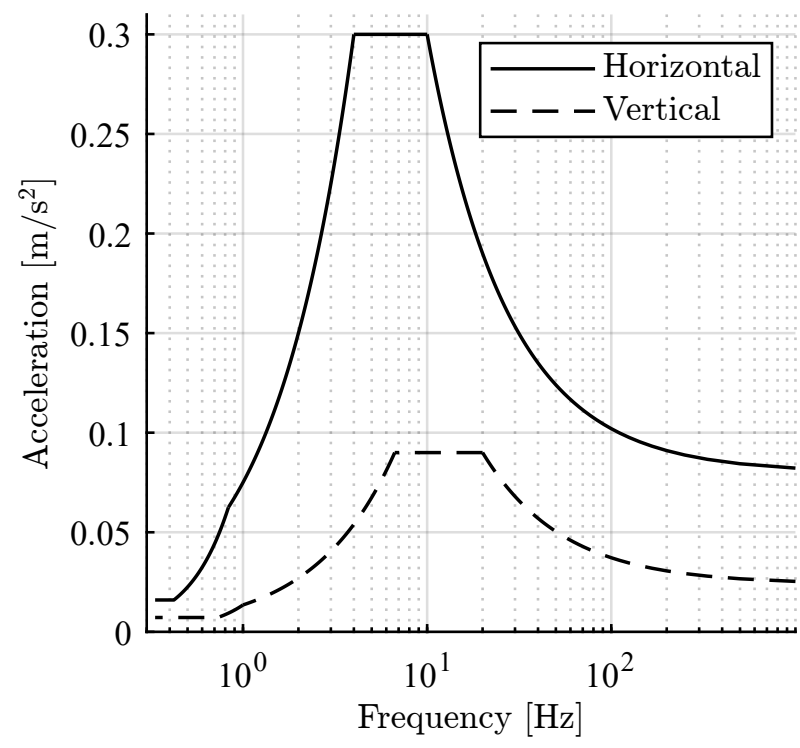

Figure 4.25: Input acceleration spectrum for the response analysis, showing acceleration magnitude as a function of frequency, for the horizontal and vertical directions. Values are taken from [81] and represent the earthquake spectrum for an area with a dense or medium-dense sand, gravel or stiff clay soil. The magnitude of the used curves corresponds to a 'weak', or scale III, earthquake on the Modified Mercalli Intensity scale [82].

Figure 4.26: Maximum von Mises stress in the cold mass and pillars during a worst-case earthquake within the frequency band given by Figure 4.25.
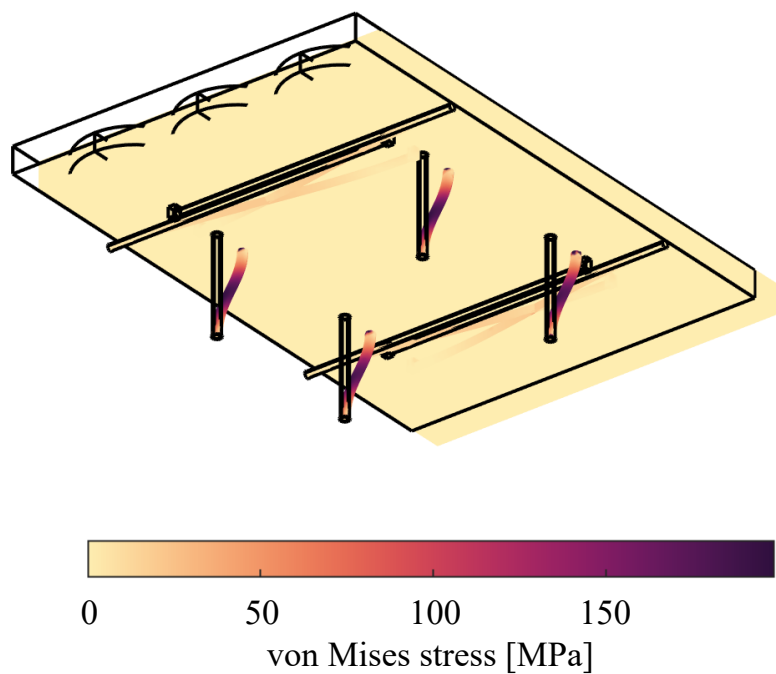


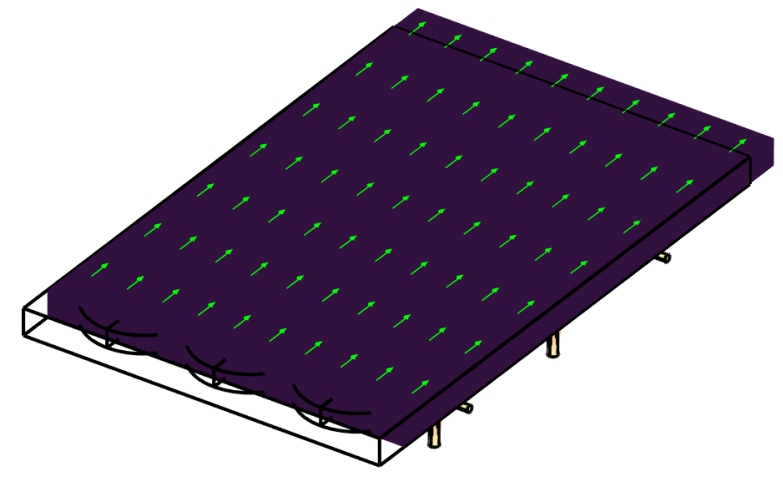

The conclusion is that the cold mass will likely not survive a weak earthquake if the cryostat itself moves as a rigid body in phase with the ground. This is because of the low stiffness in the $y$-direction due to a lack of support in this direction. The addition of a vibration isolator such as rubber between cryostat and floor possibly can improve the seismic behaviour. This is the focus of the next section.

\subsubsection{Feasibility of vibration isolation}

Intuitively placing the cryostat on the floor via a vibration isolator such as rubber pads or air cushion may help reducing vibrations. The goal of this analysis is to find out if it possible to reduce the maximum load on the G11 pillars due to vibration so that their total load does not exceed half the buckling strength per pillar of $10 \mathrm{kN}$. The gravitational load occupies $1.3 \mathrm{kN}$ per pillar of the allowed $5.0 \mathrm{kN}$. This means that a factor 7 reduction of the dynamic load as estimated in the previous section is required. As an extra margin, the following calculations aim for a factor ten reduction.

A common way to represent a vibration isolator placed between a mass and ground is by Voigt's model [80]. In this model, a spring $k$ and damper $c$ are placed in parallel between mass and ground, as illustrated in Figure 4.28.

The isolator acts as a low-pass filter. The sharpness of the resonance peak of the isolator depends on the damping ratio $\zeta$ of the system, defined as $\zeta \equiv$ $c /(2 \sqrt{\mathrm{km}})$. This ratio is between 0.01 and 0.5 for rubbers and 0.01 and 0.05 for 


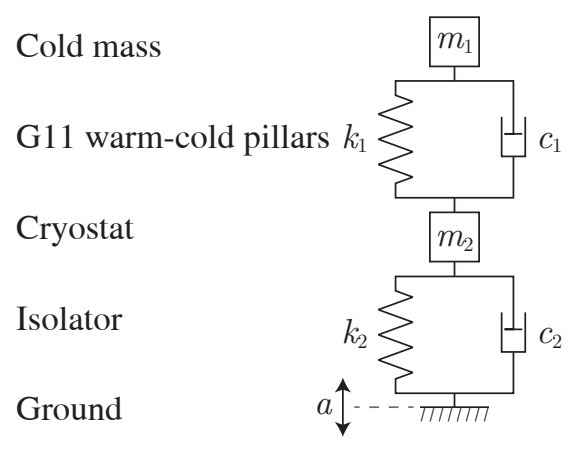

Figure 4.28: Lumped model representation of the cold mass as a mass $m_{1}$ $\left(520 \mathrm{~kg}\right.$ ) connected to the rigid cryostat (mass $m_{2}$ of $1200 \mathrm{~kg}$ ) which is in turn connected to ground accelerating at a rate a. The connections are modelled as a damper in parallel with a spring. This type of isolator is known as the KelvinVoigt model [85]. Depending on the choice of stiffness of the spring $k_{2}$ and the damping coefficient $c_{2}$ of the damper, the cryostat may not vibrate at the same amplitude and phase as the ground, possibly affecting the movement of the cold mass.

air springs [80]. If $\zeta$ is smaller than 1, a resonance peak is present.

The G11 pillar structure itself can also be modelled as a vibration isolator with a low damping capacity. To achieve this, a damper is placed parallel to the spring representing the pillars, see Figure 4.28. This damper is required to prevent a very fast rising resonance peak.

The system consists of two low-pass filters in series. The goal is to have the vibration isolator's own resonance frequency below that of the resonance frequencies of the pillar-cold mass system. In this way external excitations with a frequency content that includes the resonance frequencies of the G11 pillar-cold mass combination has a smaller effect on the cold mass, as these frequencies are damped by the isolator.

Figure 4.29 shows the magnitude of the acceleration of the cold mass scaled to that of the ground. The system without an isolator is especially responsive near the resonance frequency (Eq. 4.16).

When an isolator is employed, the peak reduces in magnitude and shifts to slightly higher frequency. A factor 10 reduction of the height of the peak around 6 to $7 \mathrm{~Hz}$ can be indeed achieved. However, the cold mass now suffers from the response to a second resonance peak originating from the resonance frequency of the isolator. At this resonance peak cryostat and cold mass move in phase. The isolator needs to have a low stiffness to push its resonance frequency below $1 \mathrm{~Hz}$. 
Below this frequency, the disturbance spectrum is much smaller and thus is less importance, see Figure 4.25..

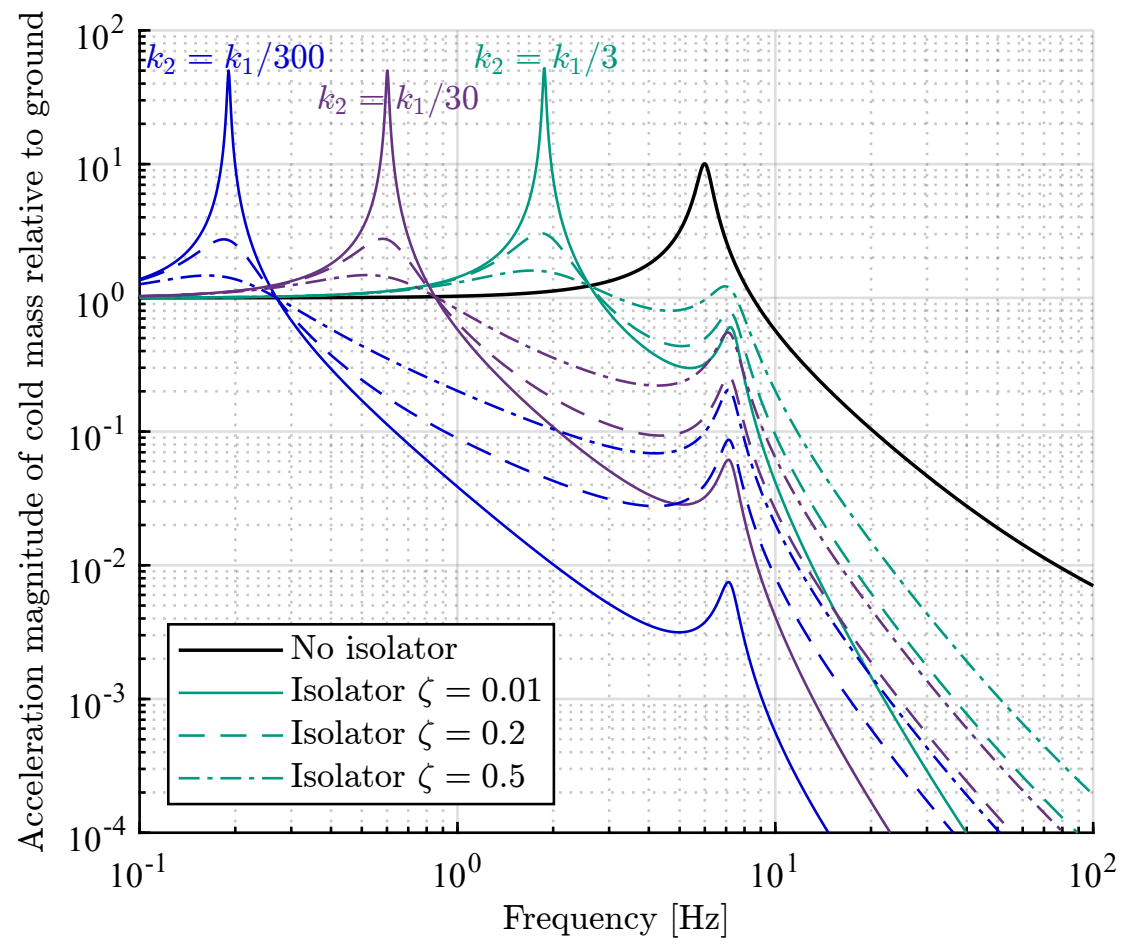

Figure 4.29: Cold mass acceleration magnitude relative to the ground, as a function of frequency. Different colours denote different stiffnesses $k_{2}$ of the isolator and different line-styles indicate different damping coefficients $\zeta$.

To estimate the deflection of the isolator when the magnet is installed, we assume a linear relation between deflection and load (allowing Hooke's law), and a spring constant $k_{2}=k_{1} / 30=2.5 \cdot 10^{4} \mathrm{kN} / \mathrm{m}$ :

$$
\Delta z=\frac{\left(m_{1}+m_{2}\right) g}{k_{2}}=\frac{1720 \cdot 9.81}{2.5 \cdot 10^{4}} \mathrm{~m}=0.68 \mathrm{~m} .
$$

This is large for a practical implementation ${ }^{10}$, however a realistic rubber- or airbased isolator need not behave linearly [80].

Also, whereas isolating the cryostat from the ground may help in reducing vibrations travelling via the ground to the cold mass, the opposite is also true. Any

\footnotetext{
${ }^{10} \mathrm{Also}$, safety concerns arise due to the stored energy of $5.8 \mathrm{~kJ}$ in such a spring: if the top-heavy cryostat slides of the isolator, the spring is able to launch a $70 \mathrm{~kg}$ human $8 \mathrm{~m}$ in the air.
} 
vibration caused by an external force on the cold mass, e.g. something touching the cryostat or a changing magnetic force on the cold mass, will have to be damped internally by the system.

To conclude, the recommendation is to position the demonstrator magnet not in an environment with strong vibrations, due to the low resonance frequencies of the cold mass, which are hard to dampen.

For the design of future MDS magnets regarding to vibrations and shocks, two aspects are noteworthy. The first is that a stronger support in the $y$-direction can be included in the mechanical design from the onset. The second is that for magnets operating at higher temperatures by using high-temperature superconductors in the coils, the extra heat budget allows for a much more rigid support structure, and thus an upwards shift of the resonance frequencies.

\subsection{Conclusion}

THE chapter dealt with the mechanical design of a conduction-cooled NbTi-

1 based MDS demonstrator magnet. The cryostat of the system has a main vessel housing the cold mass and a turret in which a cryocooler and other services are placed. The novelty of the cryostat is that it has a large flat surface facing the ferrofluid, in order to minimize the distance between ferrofluid and coils. This $50.5 \mathrm{~mm}$ distance between the cryostat and the top surface of the coil is made up from components listed in Table 4.1.

The top plate of the cryostat is supported by room-temperature stainless steel columns passing through holes in the cold mass. They allow the top plate to be relatively thin $(20 \mathrm{~mm})$. This rod structure is advantageous for more powerful MDS magnets as well, despite the added complexity in cold mass construction and cryostat. By opting for conduction-cooling of the cold mass, a double-walled cryostat is not necessary. The performance of the demonstrator is a factor 2 higher than that of a bath-cooled system that does not use the column structure.

Several concepts for cold mass design were presented. The selected design uses a cassette consisting of two aluminium-alloy machined plates that shrink-fit around the coils during cool-down and provide a compressive force on the winding pack. Underneath the cassette, high-purity aluminium heat drains ensure that the thermal gradient across the cold mass is smaller than $100 \mathrm{mK}$. It was found that the bolts that connect the two cassette halves gain a significant tensile stress of up to 140 MPA during cool-down, due to differential thermal shrinkage.

The main factor determining the minimum cassette thickness, $12 \mathrm{~mm}$ per plate for the demonstrator, is the amount of pre-compression required to prevent positive transverse stress in the winding pack due to the Lorentz force. The effects of 
the thickness on the longitudinal stress in the winding pack and on the von Mises stress in the cassette itself are shown to be secondary importance.

The cold mass is kept in place using a fibreglass pillar and tie rod structure, the dimensions of these were optimized to minimize heat in-leak. The support structure can handle both compressive and tensile loads, without yielding or buckling. G11 is found to be the optimum material choice to withstand buckling with a minimum heat in-leak.

The response of the magnet to shocks and vibrations is analysed by calculating the resonance frequencies of the cold mass-pillar structure and using modal superposition. The result is that the vertical support pillars are in risk of breaking in the case of a seismic event. The possibility of protecting the magnet using a vibration isolator such as rubber or an air cushion is shown to be possible, though care in the design of the insulator needs to be taken. 


\section{5 | Thermal \& electrical design}

In this chapter the focus is on thermal and electrical design aspects of the MDS demonstrator magnet. First the thermal design is presented, which shows that the magnet can operate using a single cryocooler with sufficient margin. The coils are conduction-cooled and operates below $4.5 \mathrm{~K}$. High-purity aluminium heat drains enable a low thermal gradient across the cold mass.

The current path is introduced, as well as the adopted protection scheme. The magnet's stored energy can safely be dumped in the coils. Diodes are placed (anti-)parallel to the coils to prevent high terminal voltages. In the case of a quench in the superconducting part of the current leads or an external anomaly, a switch is opened and the current is forced through a heater in series with the diodes, causing a deliberate transition of the coils to the normal state and thus a fast ramp-down. 


\subsection{Introduction}

THE coils in the MDS demonstrator need to operate at a temperature of $4.5 \mathrm{~K}$ 1 or lower in order to allow operation with a temperature margin of $2.0 \mathrm{~K}$, and the magnet operating at $300 \mathrm{~A}$. A procedure that ensures a safe shut-down in the case of an anomaly also needs to be included in the design. In this chapter these thermal and electrical aspects are dealt with. The layout of the chapter is as illustrated in Figure 5.1:

- First, a thermal overview of the system is given. This includes the calculated heat loads at the two stages of the cryocooler and the expected thermal gradient between cryocooler and coils.

- Second, the layout of the current leads and electrical connections are presented. The feasibility of the quench protection method for this system is explored, as well as the ability of a fast ramp-down in the case of an external anomaly. This ramp-down can be started by means of a manual trigger or by automatic actuation after detection.

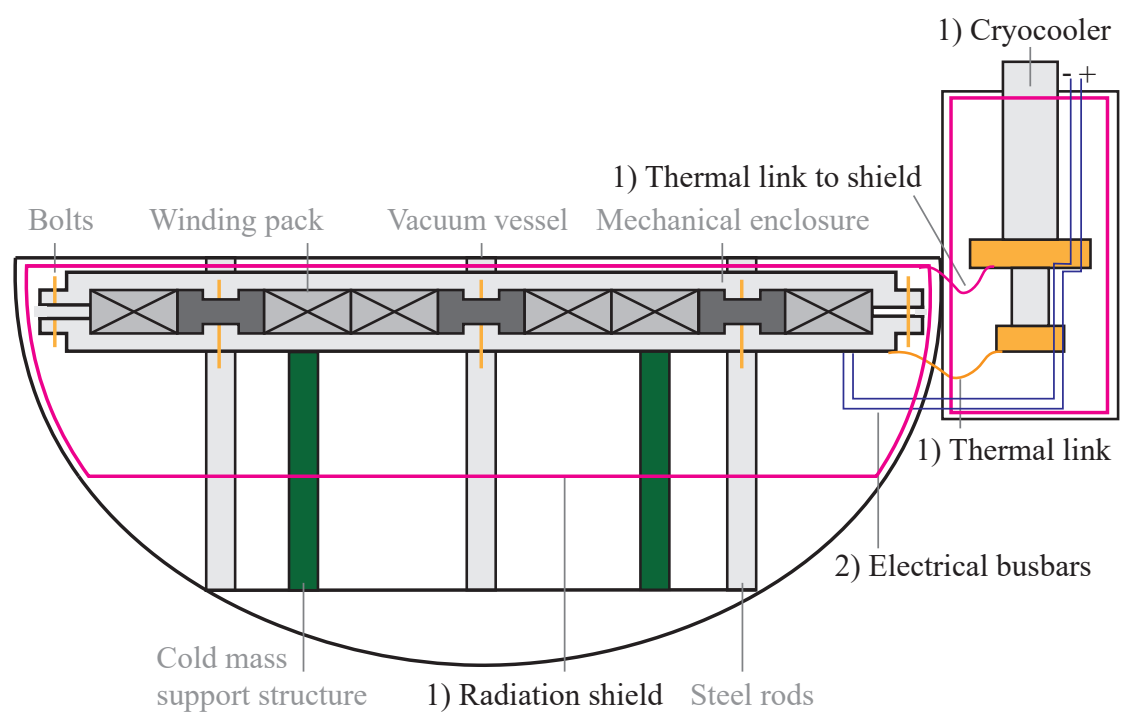

Figure 5.1: Schematic overview of thermal (1) and electrical (2) components of the magnet system as discussed in this chapter. The cryocooler has been displaced relatively to the cold mass for simplicity. 


\subsection{Thermal design}

THIS section concerns the thermal design of the magnet. This includes the 1 minimization of the heat in-leak from various sources on the cold mass and on the radiation shield, as well as minimizing the temperature difference between the warmest spot in a coil and the second stage of the cryocooler.

The coils are designed to operate with a maximum temperature of $4.5 \mathrm{~K}$, allowing a temperature margin of $2.0 \mathrm{~K}$. Since the cooling power of the cryocooler is temperature dependent, the heat in-leak to the cold mass can not be too high since otherwise the temperature of the second stage of the cooler will be above the acceptable value. This acceptable value is significantly lower than $4.5 \mathrm{~K}$, since a thermal gradient between the coils and the cryocooler is unavoidable.

Heat transfer due to convection will be regarded as negligible due to the vacuum inside the cryostat. What remain to be considered are radiation and in-leak via conduction, ramping losses in the superconductor, as well as joule heating in resistive parts of the electrical circuit.

The section starts with introducing the components that contribute to a significant heat load into the system. Heat in-leak due to conduction is discussed, as well as an estimate of the radiation load. These estimates are based on proper heat-sinking using an intermediate temperature of $77 \mathrm{~K}$.

After the identification of relevant heat loads, a lumped thermal model is solved to find the magnitudes of the heat loads as well as the temperatures of the cryocooler, radiation shield and cold mass. The model also allows an estimation of the cool-down time of the system. The cool-down is performed by the cryocooler without the aid of additional cryogens. Whereas using liquid nitrogen to speed up the cool-down process is possible, it was decided not to do so for the demonstrator, in favour of simplicity. For the same reason removable heatlinks - high-thermal conductivity links that can be mechanically disconnected at a certain point in time-between the intermediate stage and the cold mass are not considered.

Using the results of the lumped model, an estimate of the thermal gradient in the cold mass is made using a 3D FEM calculation. This allows for a prediction of the warmest spot in the coils.

\subsubsection{Cryocooler}

The cryocooler is a model RDK-415D2 two-stage Gifford-McMahon cooler from Sumitomo Heavy Industries, Ltd. The first stage has a cooling power of several tens of watts around liquid nitrogen temperature $(77 \mathrm{~K})$ and the second stage has around a watt of cooling power at liquid helium temperature $(4.2 \mathrm{~K})$. The cooling power depends on the temperature of the respective stages, as can be seen 
in Figure 5.2. The two stages are two circular copper flanges to which connections can be made. For the operating principle of such a cryocooler the reader is referred to [86].

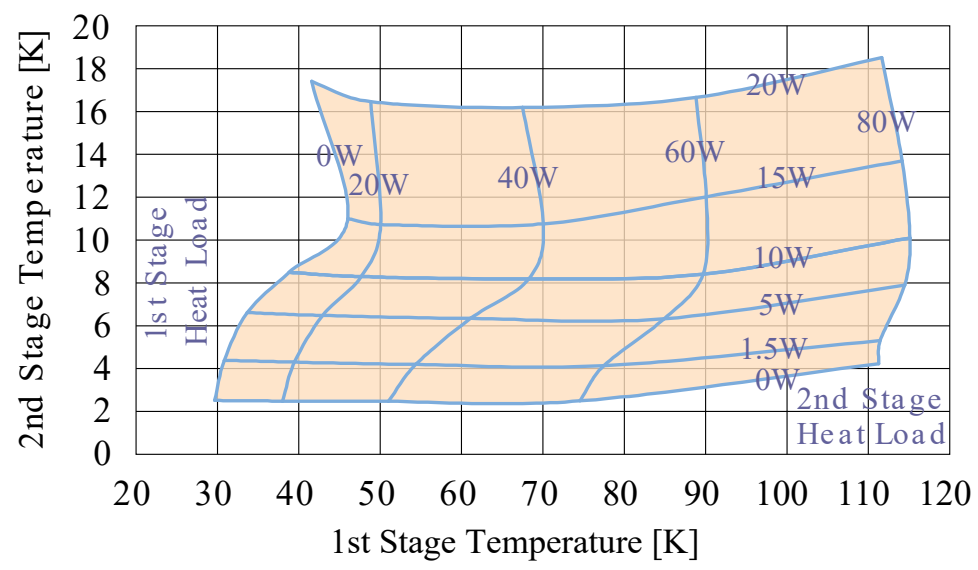

Figure 5.2: RDK-415D Cold-head capacity map at $50 \mathrm{~Hz}$ input power. Figure reproduced with data from SHI Cryogenics Group [52]. In the plot the "horizontal" lines are at constant load at the second stage and the "vertical" lines are at constant load at the first stage.

The powerful first stage is commonly used to intercept heat coming from room temperature to the cold mass. To intercept radiation from the room-temperature vacuum vessel, a heat shield made from copper and aluminium, is placed around the cold mass, the support structure, the current leads and the cryocooler.

Instead of solving the coupled problem straight away, a segregated approach is followed in which the heat load is first calculated using rough estimates for the two cryocooler stage temperatures. Here, liquid nitrogen and liquid helium boiling temperatures at atmospheric pressure are taken as a starting point, since data on thermal properties at these temperatures are readily available [39, 42].

The associated heat load is then used to calculate the cryocooler first- and second stage temperatures. In the following section the estimates are then refined by solving the lumped thermal model.

\subsubsection{Heat loads}

Several components that introduce a significant conductive heat in-leak can be distinguished. 


\subsubsection{Support structure}

The mechanical support structure keeps the cold mass in place within the cryostat. This support structure exhibits the conflicting requirements of low thermal conductance and high mechanical strength. The optimization of the chosen fibreglass structure is described in Section 4.5.

The estimated heat in-leak on the cold mass is $148 \mathrm{~mW}$ for the four vertical hollow pillars and $38 \mathrm{~mW}$ for the four horizontal solid pillars. Here the dimensions of the pillars are taken as discussed in Section 4.5.2. The pillars are heat-sunk to the cryocooler's first stage via the radiation shield to reduce the inleak at the cold mass. The heat load on the radiation shield by thermalization of the pillars is $1.1 \mathrm{~W}$ for the vertical pillars $\left(0.113 \mathrm{~m}\right.$ thermal path, $9.0 \mathrm{~cm}^{2}$ crosssectional area) and $1.0 \mathrm{~W}$ for the tie rods $\left(0.17 \mathrm{~m}\right.$ thermal path length, $12.6 \mathrm{~cm}^{2}$ cross-sectional area).

\subsubsection{Radiation}

The inner surface of the cryostat's outer vessel has an area of approximately $3.9 \mathrm{~m}^{2}$, and the radiation shield an area of $3.4 \mathrm{~m}^{2}$. The radiation shield is shown in Figure 5.3.

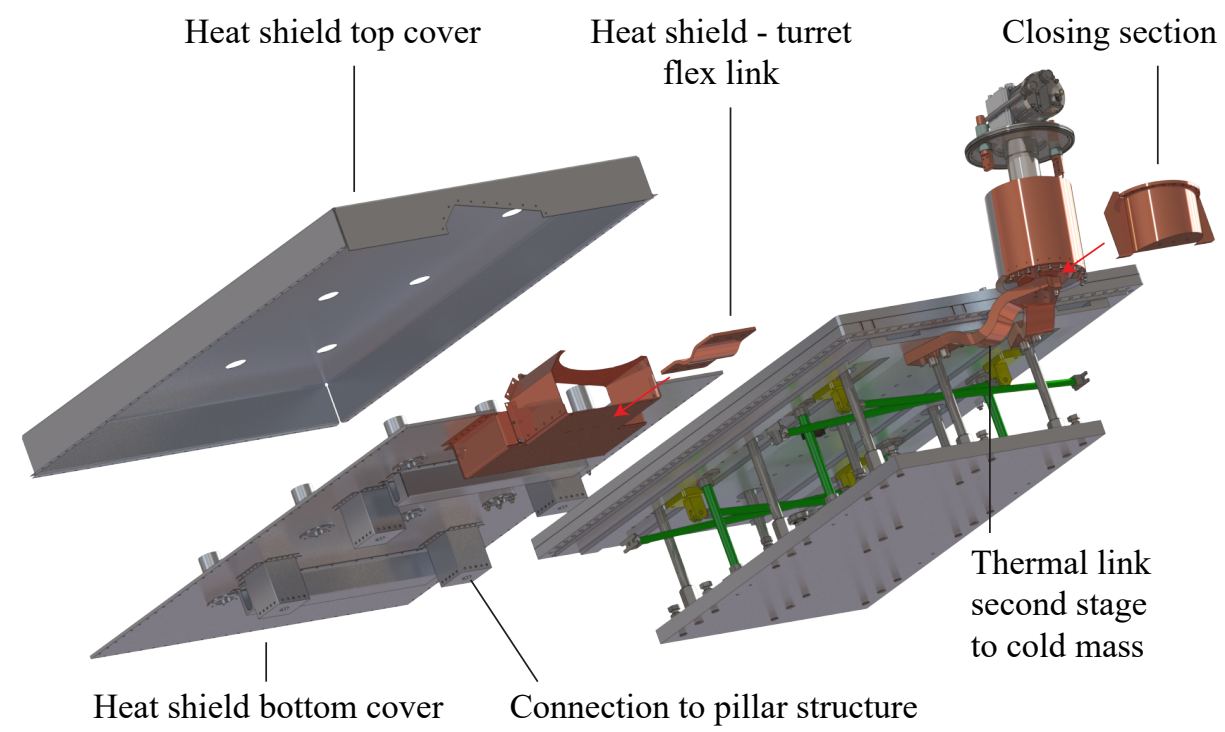

Figure 5.3: Exploded view of cold mass with radiation shield. Not shown are MLI blankets.

Copper is used for parts of the radiation shield that see a relatively high heat 
flux and where space is limited, whereas aluminium is used for the other parts to reduce weight and costs. The main structural component of the radiation shield consists of a $10 \mathrm{~mm}$ plate of AL2024-T351, which is placed below the cold mass. This type of aluminium was chosen because of availability, it has a thermal conductivity at $77 \mathrm{~K}$ of $90 \mathrm{~W} / \mathrm{m} / \mathrm{K}$, which is average compared to other aluminium alloys [87]. The plate is kept in place via the vertical pillar heat-sinks, which are glued to the pillars with Stycast 2850FT. These heat sinks consists of $10 \mathrm{~mm}$ thick plates of AL1060 and are connected to the main plate via $2 \mathrm{~mm}$ sheets of AL1060.

The top cover of the radiation shield is also made from $2 \mathrm{~mm}$ thick AL1060. The connection between parts is with bolts. Threads are vented to prevent the creation of pockets of trapped air during de-pressurisation of the system. The transfer of heat from shield to cryocooler is achieved with copper pieces. Attached to the bottom main plate is a $3 \mathrm{~mm}$ thick copper plate section. This section and previously mentioned components are assembled around the cold mass before insertion into the cryostat.

On the top of the cryocooler's first stage a copper hollow cylinder with $10 \mathrm{~mm}$ thickness is attached via M6 bolts before mounting the cooler in the cryostat turret. After installation of the coils/cold mass and cooler, the radiation shield is closed by installing a second copper hollow cylinder of $10 \mathrm{~mm}$ thickness and two side plates, indicated as "closing section" in Figure 5.3.

This cylinder is then connected with the copper plate section of the radiation shield via a flexible copper connection, consisting of $155 \mathrm{~mm}$ wide, $0.2 \mathrm{~mm}$ thick OFHC copper foils that together form a $10 \mathrm{~mm}$ thick stack. The two outer end of the foils are press-welded to form solid terminals. In this way, a flexible component is obtained that allows bending in one direction. This movement by bending allows stress-free movement of the cold mass relative to the cryocooler during cool-down. The link is annealed at $300{ }^{\circ} \mathrm{C}$ for $6 \mathrm{~h}$ in a vacuum environment to increase the thermal conductivity.

Before installation of the radiation shield, the cold mass is first wrapped in one blanket of MLI ${ }^{1}$ made by RUAG Space, with a blanket thickness of $3 \mathrm{~mm}$. The radiation shield itself is wrapped in three blankets of the same type of blanket.

The heat load due to thermal radiation between two surfaces with MLI in between is approximately $2.5 \mathrm{~W} / \mathrm{m}^{2}$ for $300 \mathrm{~K}$ to $77 \mathrm{~K}$ and $40 \mathrm{~mW} / \mathrm{m}^{2}$ for $77 \mathrm{~K}$ to $4.2 \mathrm{~K}$ [38]. This gives a heat in-leak of $9.75 \mathrm{~W}$ on the heat shield and $136 \mathrm{~mW}$ of the cold mass.

\footnotetext{
${ }^{1} M L I$, or multi-layer-insulation, consists of thin high-reflectivity layers with spacer layers between them [42]. The spacer layers minimize solid conduction across the stack. The layer density is usually around 30 layers per centimetre. MLI is placed between the hot and cold parts of a cryostat to reduce radiative heat transfer.
} 


\subsubsection{Current leads}

The operating current of the magnet of $300 \mathrm{~A}$ is fed from room temperature to the thermalization point on the cryocooler's first stage via copper current leads. These current leads are divided into two distinct sections. A pair of copper leads carrying current between $300 \mathrm{~K}$ and $77 \mathrm{~K}$ ideally ${ }^{2}$ has a shape factor of $0.274 \mathrm{~mm} / \mathrm{m} / \mathrm{A}$ and an in-leak of $45.4 \mathrm{~mW} / \mathrm{A}$ [39]. This means a heat load of $27 \mathrm{~W}$ for a pair of 300 A leads. The shape factor means that one has the freedom to find a practical length of the current lead, and base the cross-sectional area on this length. Here this section is made from braided copper wire with an RRR of 30 .

The transfer of the current from the first stage to the second stage is achieved via BSCCO-2223 current leads made from Sumitomo type G conductor. The superconducting section has a silver-gold alloy matrix ( $5.4 \%$ gold by weight) for its reduced thermal conductivity relative to a pure-metal matrix [88]. A pair of current leads consists of 6 tapes with a length of $0.16 \mathrm{~m}$ each and a cross-sectional area per tape of $4.3 \mathrm{~mm}$ by $0.23 \mathrm{~mm}$. This introduces a load of $125 \mathrm{~mW}$ at the cold end, assuming a $77 \mathrm{~K}$ first stage temperature. Additionally, the tapes are mechanically supported by a $2 \mathrm{~mm}$ thick G11 sheet per lead causing an extra heat-leak of $6 \mathrm{~mW}$.

The BSSCO tapes have a critical current $I_{\mathrm{c}}$ per tape at self-field of $291 \mathrm{~A}$ at $66 \mathrm{~K}$ and $192 \mathrm{~A}$ at $77 \mathrm{~K}$. They are situated in a low-field region $(<100 \mathrm{mT})$, thus no strong correction is needed because of the background field [88]. This yields a margin of 2 to 3 in $I / I_{\mathrm{c}}$ when the leads are anchored to the second stage at $77 \mathrm{~K}$ or $66 \mathrm{~K}$, respectively.

Part of the current leads are shown in Figure 5.4. One can see the vacuum feedthroughs with a relatively large cross-sectional area to make sure the vacuum seals are at room-temperature. Also shown are the heat-sinks to the first- and second stages, and the superconducting connection between the two stages. The copper connection between the vacuum feedthrough and the first stage heat sink is not shown. The current flows from the vacuum feedthrough to the not-shown copper current lead via a soldered connection. A close-up of the first stage is shown in Figure 5.5.

The lead is soldered to a copper rod and copper plate combination. This plate is bolted to the heat-sink with an indium foil in between. The heat-sink consists of a copper main ring, making contact with the cryocooler flange. A second, Cshaped, piece of copper is glued to this ring. G11 insulation pieces of $0.2 \mathrm{~mm}$ thickness provide electrical isolation. The current flows through the $\mathrm{C}$-shaped part. At the other end of the $\mathrm{C}$, the superconducting lead is bolted using a copper

\footnotetext{
${ }^{2}$ Valid for conduction-cooled leads. Balancing the Joule heating with the in-leak through conduction yields an optimum cross-sectional area for a given length over which the current is transported between two thermal anchors [39].
} 


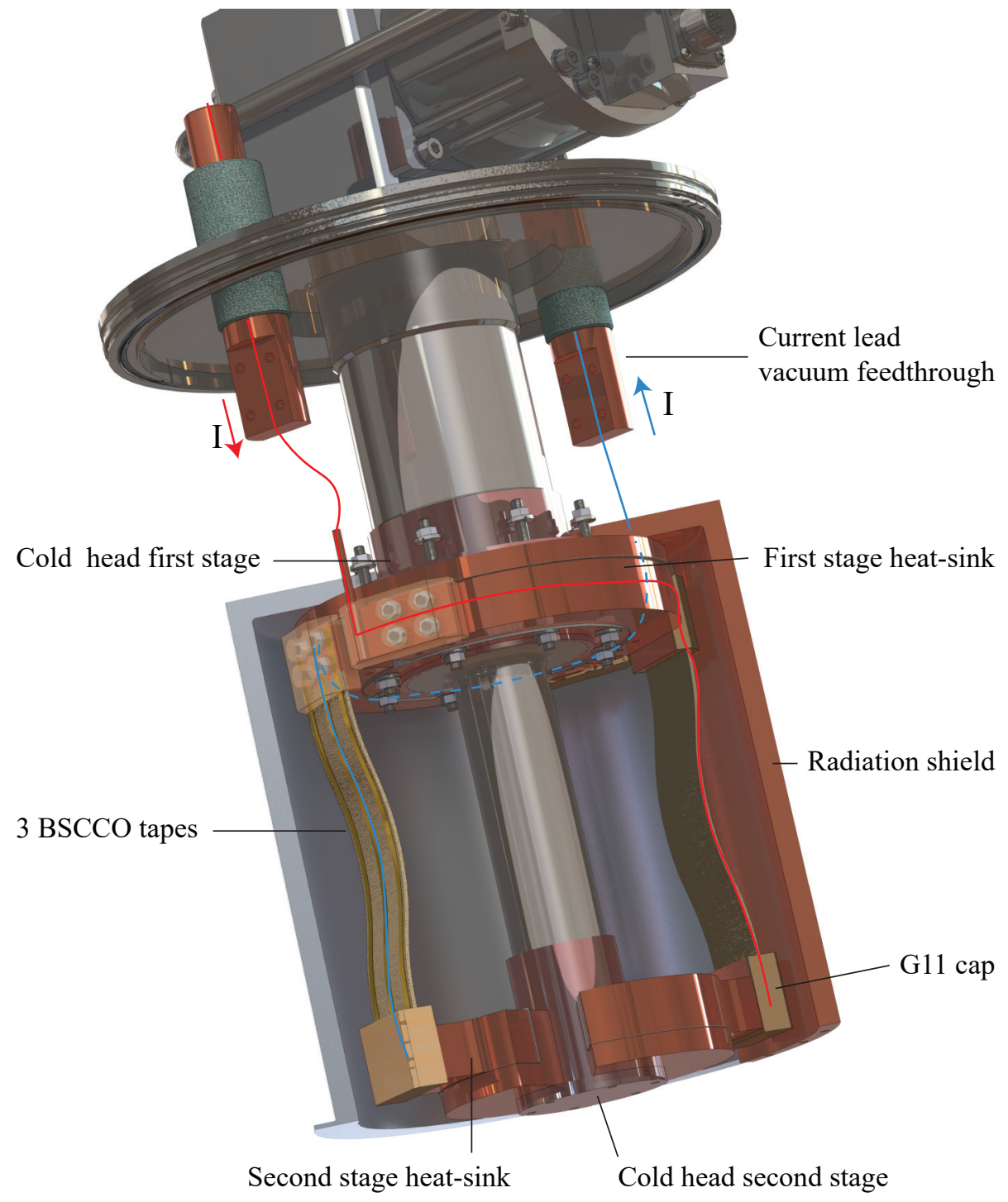

Figure 5.4: Current leads and heat-sink. The current path going in and out of the set-up is sketched in red and blue. 


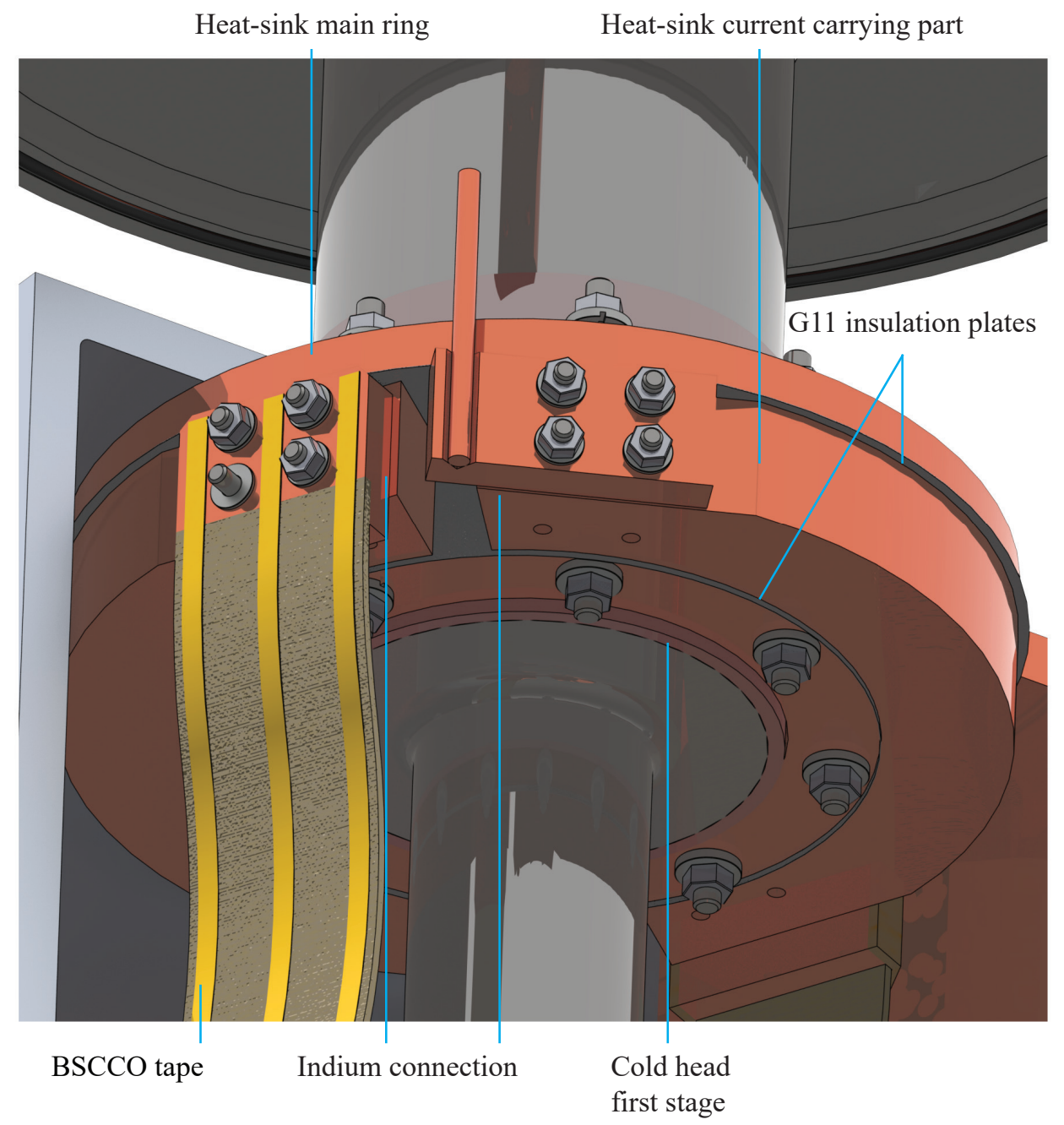

Figure 5.5: View of the first-stage heat-sink. Not shown are the connections between the vacuum feedthrough and the copper rod, G11 insulation caps and the MLI layer. 
plate-indium-copper connection. G11 caps are placed over several areas over the current leads to prevent electrical contact with the MLI layer. Other parts are wrapped in Kapton foil.

The second stage heat-sink is similar to that of the first stage. The current is passed from the second stage heat-sink to the coils using NbTi conductor. Four wires with the same properties as that of the coils are placed in parallel to form a lead. Using four conductors instead of a single conductor increases the current sharing temperature of this section from $6.5 \mathrm{~K}$ to $6.8 \mathrm{~K}$. This gain is small because of the steep drop of performance of $\mathrm{NbTi}$ with increasing temperature, see Appendix A. Instead of connecting two C-shaped parts to a single main ring, as is the case for the first stage, for the second stage the main ring is replaced by two additional C-shaped parts, since otherwise mounting the part on top of the second stage flange would not be possible since the inner diameter of the ring is smaller than the outer diameter of the cold head. The bottom of the second stage flange is already allocated for the thermal link between the cooler and the cold mass, and thus not available for heat-sinking the current leads. As the heat load through this link will be shown to be larger than the load through the current leads, using the contact area underneath the second stage for the thermal link is preferred.

For the first stage heat-sink the contact area between the copper ring and the copper C-piece is $92 \mathrm{~cm}^{2}$. Using a thermal conductivity of G11 of $0.28 \mathrm{~W} / \mathrm{m} / \mathrm{K}$ at $77 \mathrm{~K} \mathrm{[42]} \mathrm{and} \mathrm{a} \mathrm{heat} \mathrm{load} \mathrm{of} 13.6 \mathrm{~W}$ per lead, a temperature difference between the two copper pieces of $1.0 \mathrm{~K}$ is expected.

The second stage heat-sink has a contact area of $59 \mathrm{~cm}^{2}$. With a heat load of $66 \mathrm{~mW}$ per lead, this gives a gradient of $31 \mathrm{mK}$ across the $\mathrm{G} 11$ ( $k$ of $0.072 \mathrm{~W} / \mathrm{m} / \mathrm{K}$ at $4.2 \mathrm{~K}$ [42]) and $22 \mathrm{mK}$ across the two Stycast interfaces ( $k$ of $0.05 \mathrm{~W} / \mathrm{m} / \mathrm{K}$ at $4.2 \mathrm{~K}[42])$. The thermal contact resistance across these interfaces is expected to be below $1 \mathrm{mK}[89]$.

\subsubsection{Instrumentation}

The wiring connecting the various sensors to the read-out instrumentation carries in a small amount of heat. As this is a demonstrator system, a larger number of sensors is desired than one may consider for a production model. Assume a sensor with four magnanin wires of $0.2 \mathrm{~mm}$ diameter connected between the thermal shield and the cold mass thermalization point. Taking an effective thermal conductivity of $6 \mathrm{~W} / \mathrm{m} / \mathrm{K}$ [42], the heat load per sensor is $56 \mu \mathrm{W}$ for a meter line (scaling with length ${ }^{-1}$ ). For 20 sensors at $4 \mathrm{~K}$ with an average wiring length of one meter between the thermalization pads, this yields a total in-leak from radiation shield to cold mass of $4.5 \mathrm{~mW}$. The Joule heating in the wires is negligible. 


\subsubsection{Ramping losses}

The hysteresis loss rate $P_{\text {hyst }}$-the power dissipated by movement of fluxoids in the superconductor due to a changing magnetic field —is estimated (from [39]) by integrating the product of the rate of change of the magnetic flux density, $\dot{B}$, and critical current density $J_{\mathrm{c}}$

$$
P_{\text {hyst }}=\frac{2}{3 \pi} \lambda_{\text {sc }} d_{\text {fil }} \int_{V_{\text {windingpacks }}} J_{\mathrm{c}}(B, T) \dot{B} \mathrm{~d} \tau,
$$

where $\lambda_{\mathrm{sc}}$ is the volumetric fraction of superconductor in the winding pack (29\%), $V_{\text {windingpacks }}$ the volume of the three winding packs $(42 \mathrm{~L})$ and $d_{\text {fil }}$ the diameter of the superconducting filaments $(75 \mu \mathrm{m})$.

The effect of transport current on the dissipation is neglected as the ratio between the current density and critical current density is low $(<20 \%)$.

As an example, we set the ramp duration of the magnet to $15 \mathrm{~h}$ with a constant (but spatially varying) $\dot{B}$. The calculated power as a function of time is presented in Figure 5.6. The dissipated power decreases over time because the critical current decreases with increasing magnetic field. The power increases with decreasing temperature because the critical current increases with decreasing temperature.

From the figure, it seems realistic to use $0.1 \mathrm{~W}$ as a heat load for the hysteresis loss for a 15 hour ramp. The effect of ramping at a higher speed is discussed in Section 5.2.5.

The relative contributions of eddy currents and coupling losses to the heat load during ramping are small compared to that of hysteresis.

\subsubsection{Cooling budget - first estimation}

With the heat loads as calculated above, a summation of the total heat loads on the first and second stages of the cryocooler can be made, as is performed in Table 5.1. The calculated heat loads at $77 \mathrm{~K}$ and $4.2 \mathrm{~K}$ are smaller than the cryocooler's cooling power at those temperatures. Thus the operation of the system using a single cryocooler looks feasible.

It is of interest to find out at which temperatures of the first and second stages of the cooler the cooling power will equal the heat load. Instead of following an iterative approach manually to find this balance, a lumped element model is solved numerically. This model and its results are presented in the next section. 


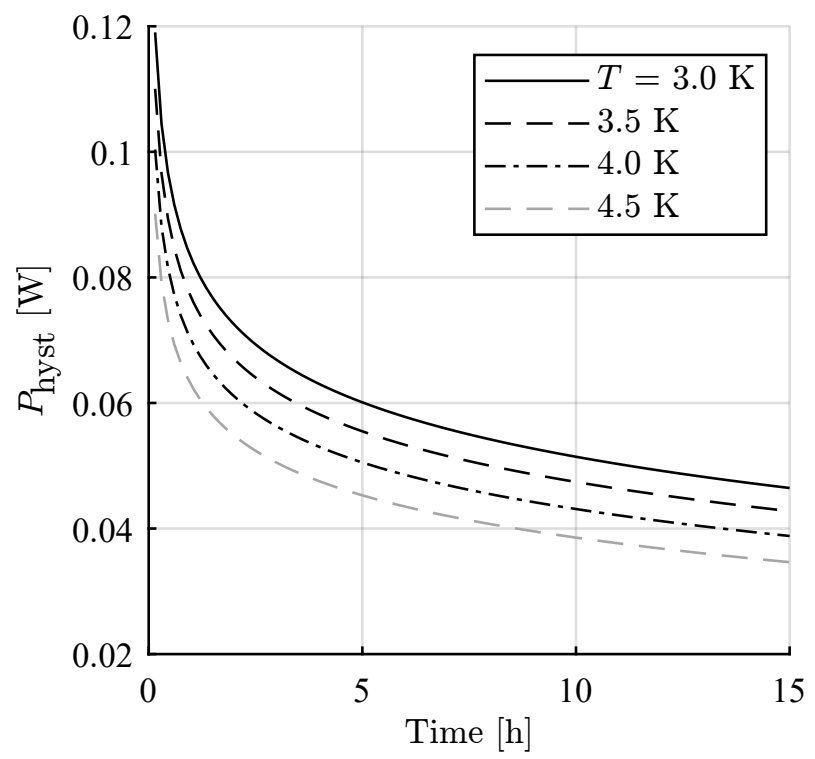

Figure 5.6: Hysteresis loss rate $P_{\text {hyst }}$ as a function of time, plotted for different temperatures of the coils for a ramp to $300 \mathrm{~A}$ in $15 \mathrm{~h}$.

Table 5.1: Cryogenic loads in conduction-cooled MDS system. The heat loads are calculated from $300 \mathrm{~K}$ to $77 \mathrm{~K}$ for the first stage and from $77 \mathrm{~K}$ to $4 \mathrm{~K}$ for the second stage.

\begin{tabular}{lll} 
& $1^{\text {st }}$ stage $\dot{Q}[\mathrm{~W}]$ & $2^{\text {nd }}$ stage $\dot{Q}[\mathrm{~W}]$ \\
\hline Current leads & 27 & 0.13 \\
Support structure & 2.2 & 0.18 \\
Thermal radiation & 9.8 & 0.14 \\
Loss in coils during ramping & 0.1 \\
\hline Total & 39 & 0.55 \\
Cryocooler SHI RDK-415D 4K & $60 \mathrm{~W}$ at $77 \mathrm{~K}$ & $1.5 \mathrm{~W}$ at $4.2 \mathrm{~K}$ \\
a As specified by the manufacturer. Measured performance by SHI with \\
60 Hz AC input are 45 W at 38 K and 1.5 W at 3.8 K [52]. At 50 Hz \\
this cooler model has approximately the same performance for the second \\
stage, and the first stage has roughly a $12 \mathrm{~K}$ higher temperature at a $45 \mathrm{~W}$ \\
load [52]. This is better than the performance in the official load-map \\
shown in Figure 5.2.
\end{tabular}




\subsubsection{Lumped thermal model}

A lumped thermal model is used to calculate the temperatures and heat flows in the system. To calculate the temperature at which the heat loads on the first and second stages of the cryocooler balance the cooling power, the cooling power as a function of temperature is required as an input. Measured data presented in [90] are used. The low-temperature measurement points are shown in Figure 5.7. The paper also includes data on the second stage cooling power up to $16 \mathrm{~K}$ [90].

Additionally, a data-point at $150 \mathrm{~K}$ is taken from [91]. At this temperature, the first stage was measured to have a cooling power of $80 \mathrm{~W}$ and the second stage of $45 \mathrm{~W}$, measured using $60 \mathrm{~Hz}$ input power. In the lumped thermal model, the interpolation between data-points is set to linear and extrapolation to constant. In this way, the cooling power above $150 \mathrm{~K}$ is thought to be underestimated. The performance differences between 50 and $60 \mathrm{~Hz}$ input power are acceptable for our use of these high-temperature data-points, which is a calculation of the cool-down time of the system.

The lumped thermal network consists of thermal masses connected via conductive links, as shown in Figure 5.8.

The considered masses are the first and second stages of the cryocooler, radiation shield and cold mass. The cold mass is considered to be at a uniform temperature; the gradient across the cold mass is subject of the next section.

The first stage is connected to a fixed room temperature node via the current leads. The current leads also connect the first and second stages. The thermal gradients between current leads and cryocooler, estimated in Section 5.2.2.3 at $1.0 \mathrm{~K}$ for the first stage and $30 \mathrm{mK}$ for the second stage, are neglected here.

The radiation shield is connected to the cryocooler via a copper link (Copper link I in the figure). This link represents the copper link as a resistor in series with a second resistor representing the gradient across the shield. The warmest point on the shield is used to calculate heat transfer from and to the shield. This includes heat flow through the fibreglass support structure to the shield from room temperature (Support structure I) and from shield to cold mass (Support structure II). The effective thermal resistance of the shield in Copper link I is matched so that the calculated hot-spot temperature matches results from a 3D FEM calculation of the radiation shield, which will be shown later.

Radiation between surfaces is taken into account by considering the geometry as two infinite parallel plates. The MLI is represented by an adjusted emissivity coefficient. The heat load due to thermal radiation between two surfaces with MLI in between is taken to be $2.5 \mathrm{~W} / \mathrm{m}^{2}$ for $300 \mathrm{~K}$ to $77 \mathrm{~K}$ and $40 \mathrm{~mW} / \mathrm{m}^{2}$ for $77 \mathrm{~K}$ 


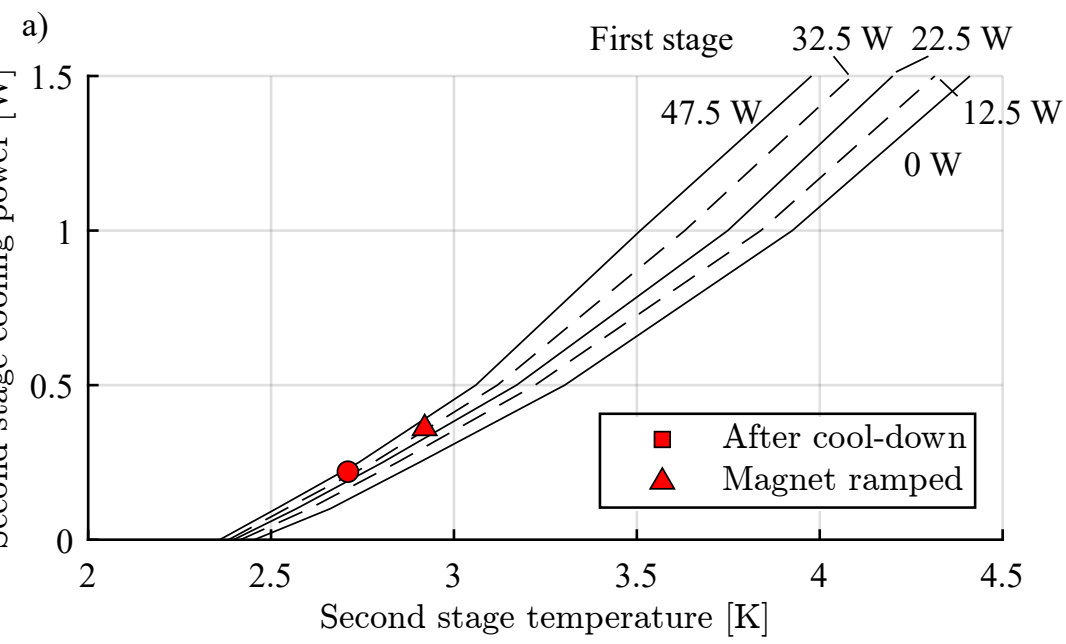

b)

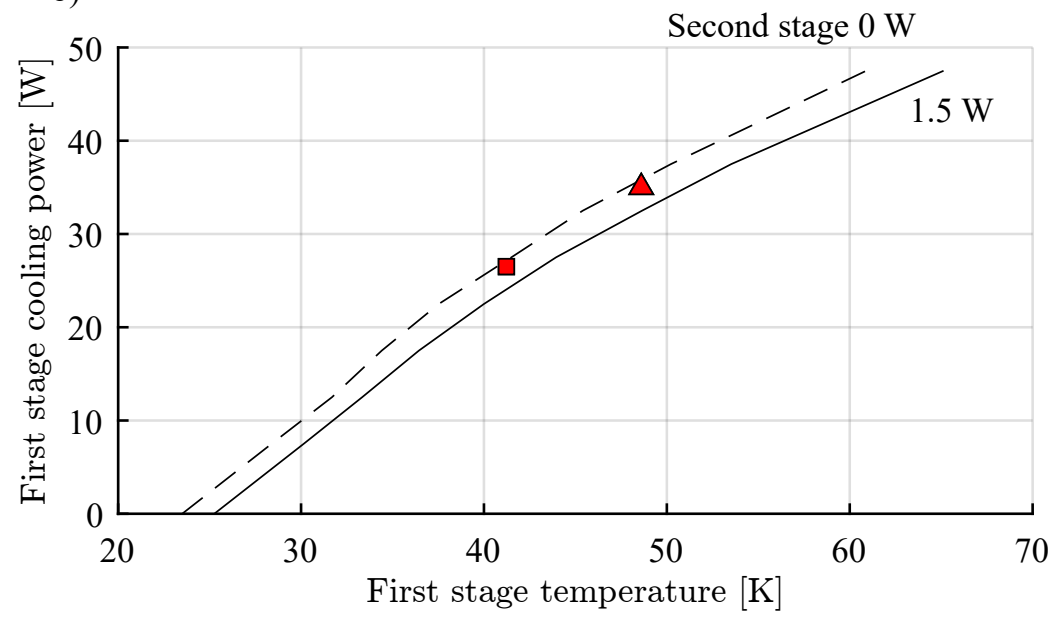

Figure 5.7: Cooling power of RDK-415D cryocooler as a function of temperature and load at neighbouring stage. Data are taken from [90]. Also indicated are the expected operating points of the MDS demonstrator magnet system as calculated using the lumped thermal model. 
a) Cool-down

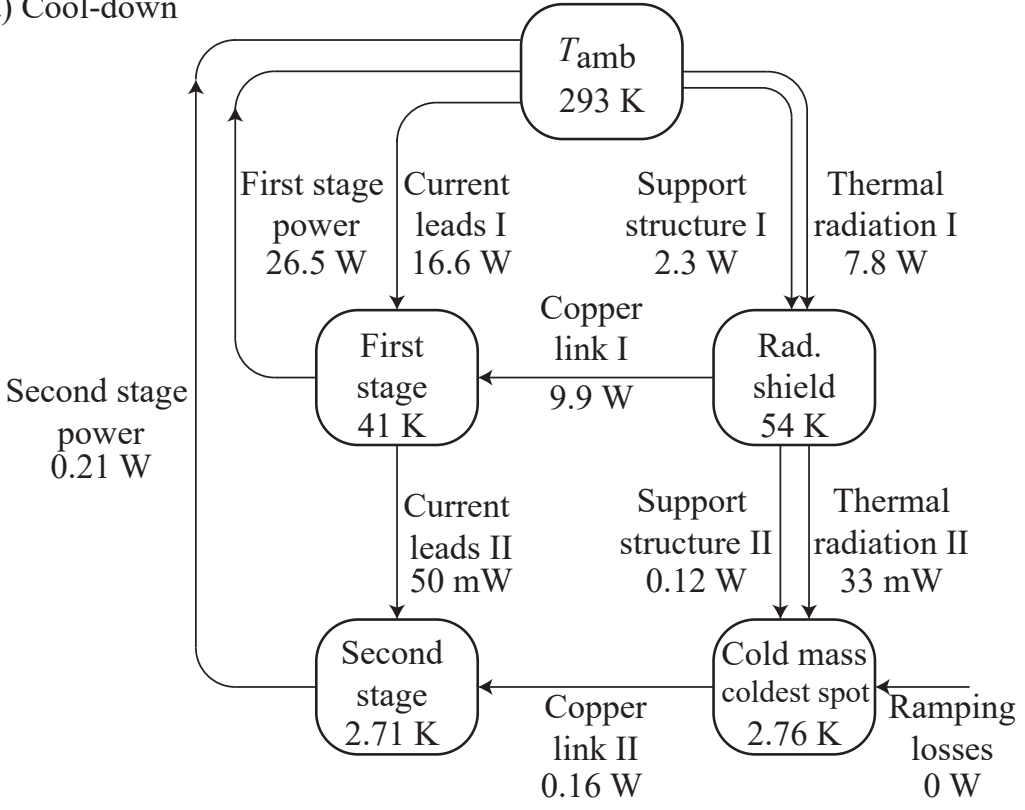

b) Magnet on

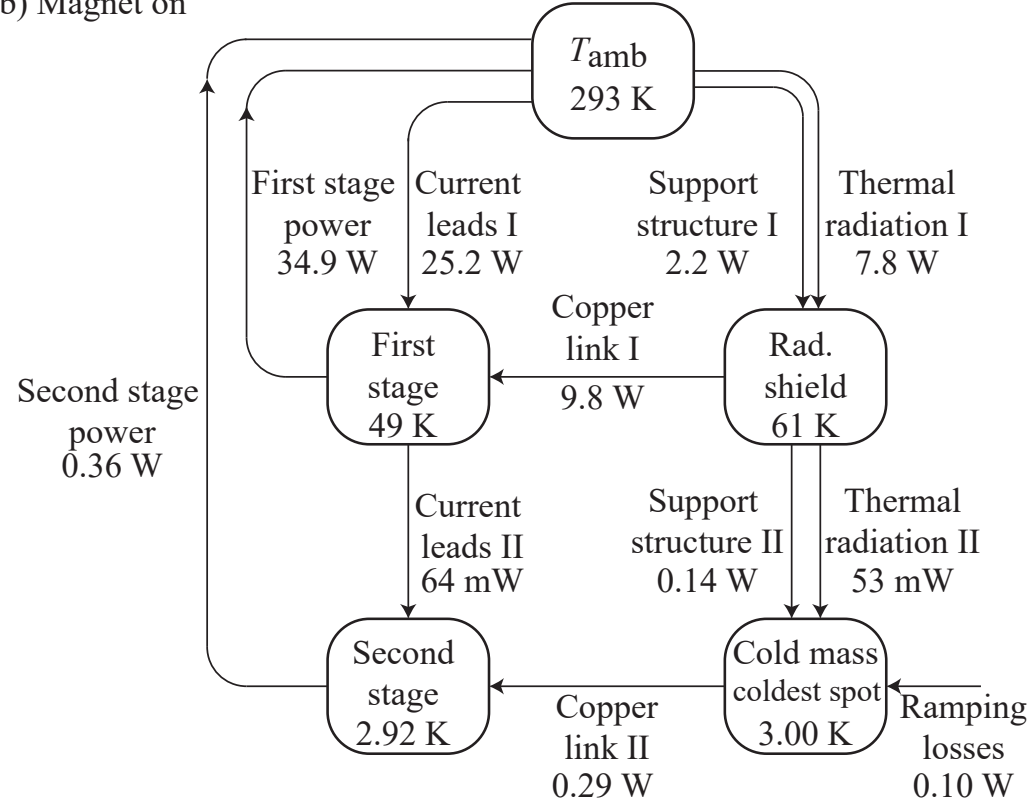

Figure 5.8: Heat flows and temperatures in lumped thermal network calculations. a) shows the situation after cool-down. b) including the effect of ramping up the magnetic field. 
to $4.2 \mathrm{~K}$ [38], respectively ${ }^{3}$. This yields an effective surface emissivity of 0.0055 for the transfer between room temperature and radiation shield (Thermal radiation I), and 0.0201 between radiation shield and the cold mass (Thermal radiation II). These emissivities are used in the parallel plate approximation to compute the radiation in the lumped model.

The support structure is modelled by dividing the pillars each in ten sections over the length. These are connected in series. This way, the effect of the nonlinear temperature profile on the thermal conductivity is taken into account. Alternatively, one could work with thermal conductivity integrals.

The second stage of the cryocooler is connected to the cold mass via a thermal link. This link consist of copper OFHC laminates of $0.2 \mathrm{~mm}$ thickness each. These are hot-pressed at their ends to form solid end-pieces, similar to the link discussed for the radiation shield. The effective cross-sectional area is $45 \mathrm{~cm}^{2}$. The flexibility resulting from a bend in the laminates allows for a stress-free cooldown of the system. The RRR of the thermal links in the calculations was set to 100 , which is a low-end value for annealed OFHC copper [42].

This link connecting the cold mass and the cooler consists of two copper parts, which when installed make up the shape of a letter V, as can be seen in Figure 5.3. Here the bottom of the $\mathrm{V}$ corresponds to the anchoring points on the cold head. This solution is chosen as it allows a connection on the cold mass on two places between the racetrack coils, where there is space to bolt the link with sufficient force $(60 \mathrm{kN}$ per link). Titanium bushings are used to prevent the pre-tension being lost on cool-down. The path-length between the middle of the cold contact area and the middle of the warm contact area of the link is taken as the effective length over which the gradient is calculated. This length is $300 \mathrm{~mm}$.

Due to the relatively small contact area between cold head and thermal link, the interface between the two must be carefully considered as thermal contact resistance at low temperatures can be significant [42]. Measurements were carried out at the University of Twente to measure the thermal contact resistance using different options for the interface [92]. It was found that an indium sheet of $0.1 \mathrm{~mm}$ thickness between a copper block and an aluminium block resulted in a contact resistance of $40 \mathrm{mK} / \mathrm{W}$ at $3.8 \mathrm{~K}$ when bolted with $29 \mathrm{kN}$. This is the interface with the lowest measured thermal contact resistance between copper and aluminium. For the copper-indium-copper connections we will assume the same thermal contact resistance. The contact area of the cold head, $30 \mathrm{~cm}^{2}$, used for the measurements is slightly smaller than that of the cold head for the MDS demonstrator, $35 \mathrm{~cm}^{2}$. Thus the results from [92] are taken as a worst-case value.

\footnotetext{
${ }^{3}$ The selected MRI blankets, type Coolcat 2 NW from RUAG Space, perform better than these initial estimates. By using 3 blankets between between $300 \mathrm{~K}$ and $77 \mathrm{~K}$, the estimated heat flux is $0.6 \mathrm{~W} / \mathrm{m}^{2}$ [50]. One blanket between $77 \mathrm{~K}$ and $4 \mathrm{~K}$ gives a heat flux of $20 \mathrm{~mW} / \mathrm{m}^{2}$.
} 
The effect of temperature at low temperatures on the thermal contact resistance $R_{\mathrm{c}}$ can be approximated by

$$
R_{\mathrm{c}}=2.56 a T^{-2.18}
$$

where $a$ has a value of one and a unit of $\left[\mathrm{K}^{3.18} / \mathrm{W}\right]$ [92].

Applying thermal grease or using a dry-metal contact resulted in a higher contact resistance [92], thus the choice of using indium sheets for the thermal contact between the cold head and the thermal link as well as the thermal link and the cold mass was made. The downside of this approach is that the link could be hard to remove from the cold head.

The link is connected to the cryocooler's second stage using six M6 bolts and a pre-tension of $7 \mathrm{kN}$ each. The thermal contact resistance is included twice in the model, in series with the V-link.

The copper V-link is situated partly in a medium magnetic field region (up to $2 \mathrm{~T}$ ). To account for this, the thermal conductivity of the link is calculated using an average magnetic field in the link of $0.5 \mathrm{~T}$, using a formula from [93] for the thermal conductivity as a function of magnetic field, temperature and RRR. The reduction in thermal conductivity due to the magnetic field is $13 \%$. The gradient across the V-link is estimated to be small enough so that a 3D FEM simulation of the thermal link taking into account the spatially-depended magnetic field is not required.

The calculated temperatures and heat flows are given in Figure 5.8. The second stage cools down to $2.7 \mathrm{~K}$ and increases to $2.9 \mathrm{~K}$ when the magnet is ramped up. The first stage cools down to $41 \mathrm{~K}$ and increases to $49 \mathrm{~K}$ when the magnet is powered. As this is lower than the $77 \mathrm{~K}$ used in the initial calculations for the thermal budget (Table 5.1), the heat flow between the first stage and second stage is also lower. As a result the cold mass eventually cools to a temperature of $3.0 \mathrm{~K}$ at the point where the thermal link is attached.

The heat load through the copper current leads is calculated using a 1D FEM model. The temperature profile of the optimized leads is shown in Figure 5.9. When carrying current between $293 \mathrm{~K}$ and $49 \mathrm{~K}$, the optimal cross-sectional area per lead for copper with a RRR of 30 was found to be $76 \mathrm{~mm}^{2}$ per meter of length. High-purity copper should be avoided, as the Wiedemann-Franz law does not hold for this type of copper below $77 \mathrm{~K}$ [42]. Brass is a second option as a material for the lead, but is not chosen as low-RRR copper was available in the laboratory.

A simplified 3D FEM calculation representing the aluminium AL2024-T351 bottom plate of the heat shield and AL1060 top cover was performed to estimate the temperature gradient across the radiation shield, see Figure 5.10. The thermal 


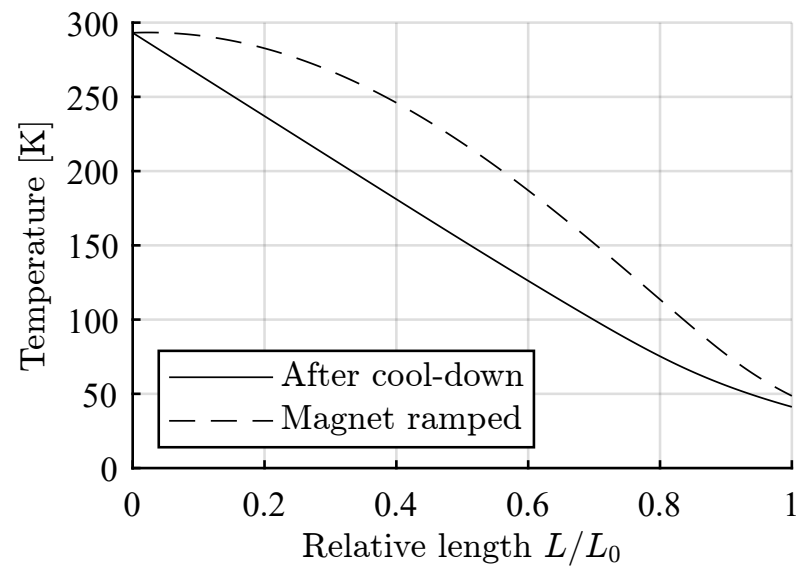

Figure 5.9: Temperature in copper current lead along the length, for a scenario in which the coils are cooled-down but not powered, and a scenario in which the current leads are carrying the full operating current of $300 \mathrm{~A}$. The main reason behind the different profiles is resistive heating in the leads.

conductivity of AL2024-T351 and AL1060 was taken from [87]. The applied heat loads are those of radiation and anchoring of support pillars as calculated in the lumped model, see Figure 5.8.

The connection to the copper parts is modelled as a small surface with a fixed temperature of $50 \mathrm{~K}$. The result of the simulation is that the warmest spot of the radiation shield is $61.5 \mathrm{~K}$. Compared to the aluminium alloys, the copper parts of the heat shield have a relatively high thermal conductivity as well as a large cross-sectional area, and thus it can be reasonable considered that the temperature gradient is small across these parts.

The hot-spot temperature of $61.5 \mathrm{~K}$ matches the lumped thermal model calculation because the value of the resistor representing the gradient across the radiation shield was chosen to match the 3D simulation result. The temperature of the radiation shield is important for the radiation towards the cold mass, as there is a $T^{4}$ scaling in the heat flux. Thus the question arises whether representing the radiation shield temperature with a single scalar in the lumped model is valid.

To answer this question a histogram of the temperature distribution of the inside surfaces of the radiation shield is shown in Figure 5.11. The histogram is weighted by multiplying the $y$-axis by a factor $T^{4}$. A major fraction of the radiation comes from around $61 \mathrm{~K}$. The mean effective temperature is calculated from the histogram to be $59 \mathrm{~K}$. Thus using the hot-spot temperature to calculate the radiation results in around $18 \%$ too high radiation load. However, the effect 


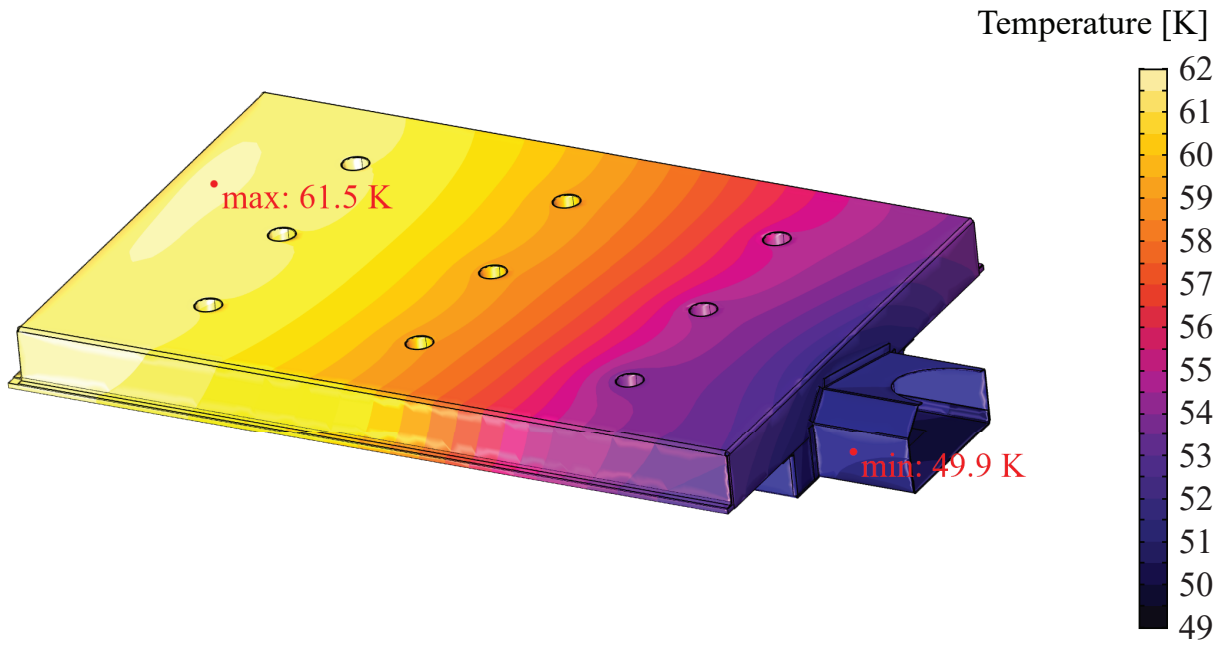

Figure 5.10: Temperature distribution in the radiation shield when the magnet has been energized.

of uncertainty in the emissivities is probably a lot higher.

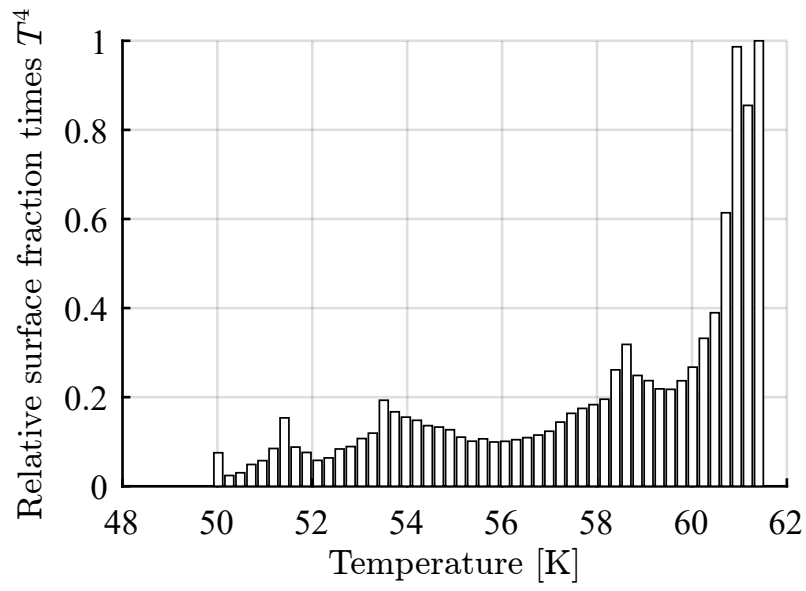

Figure 5.11: Histogram of inner surface of radiation shield after magnet energizing. The $y$-axis has been multiplied by $T^{4}$ to indicate the relative importance of each temperature in the radiative load. The y-axis is normalized. 


\subsubsection{Cold mass temperature}

In this section an estimate of the hot-spot temperature of the coils during normal operation is presented.

The lumped thermal model predicts a temperature of $3.0 \mathrm{~K}$ at the coldest spot of the cold mass. The temperature gradient between this spot and the cryocooler includes (1) the copper thermal link between the cryocooler and the aluminium heat drains on the cold mass, (2) the thermal contact resistance between the cryocooler and thermal link, and (3) between the thermal link and the aluminium heat drains. Key is how much temperature difference there will be between the coldest spot on the heat drains and the coils.

A geometry of the simulation model is shown in Figure 5.12. The whole cold mass is simulated, whereas in principle a $y z^{\prime}$-symmetry plane can be utilized. This is not done because now the same geometry can be used to simulate the effect of a point load located anywhere in the geometry. This is helpful when estimating the effect of an electrical short in the electrical circuit, as is performed in Chapter 6.

All bolts and threads are omitted from the simulation. The geometry consist of the three coils, the stainless steel yokes on which these are wound, the copper crescent spacers (RRR 100), the copper end-pieces (RRR 100), the high-purity aluminium heat drains (RRR 1500) and the two-part aluminium alloy cassette. The copper link between the cold mass and the cryocooler is modelled by placing a fixed temperature boundary condition, shown by the blue patch in the figure. The heat load from the vertical pillars is applied as well as the heat load from the tie rods, as indicated in the figure.

The winding packs are modelled as orthotropic homogeneous objects. The material properties are calculated using a winding pack composition as detailed in Section 4.3 and with thermal properties as in Table 5.2. Data for Formvar, Stycast and $\mathrm{NbTi}$ at $4.2 \mathrm{~K}$ are used. Using these values to represent the winding pack at $3.0 \mathrm{~K}$ is optimistic.

The thermal conductivity of the winding pack in the direction of the current flow is taken to be a factor 0.39 of that of copper with RRR 100 at $3.0 \mathrm{~K}$. This is because the copper occupies an estimated $39 \%$ of the winding pack volume, and because the heat flux in the direction of the conductor can be considered to flow only in the copper, as it has a much higher conductivity than the other components of the winding pack. The effect of magnetic field on the thermal conductivity is taken into account by giving the simulation access to the 3D magnetic field distribution. As mentioned in Section 3.1.2, the measured RRR of the actual conductor is 111, thus the RRR of 100 used in the calculation is considered to give a good representation. The thermal conductivity of copper as a function of $\mathrm{RRR}$, magnetic field and temperature is taken from [93]. 

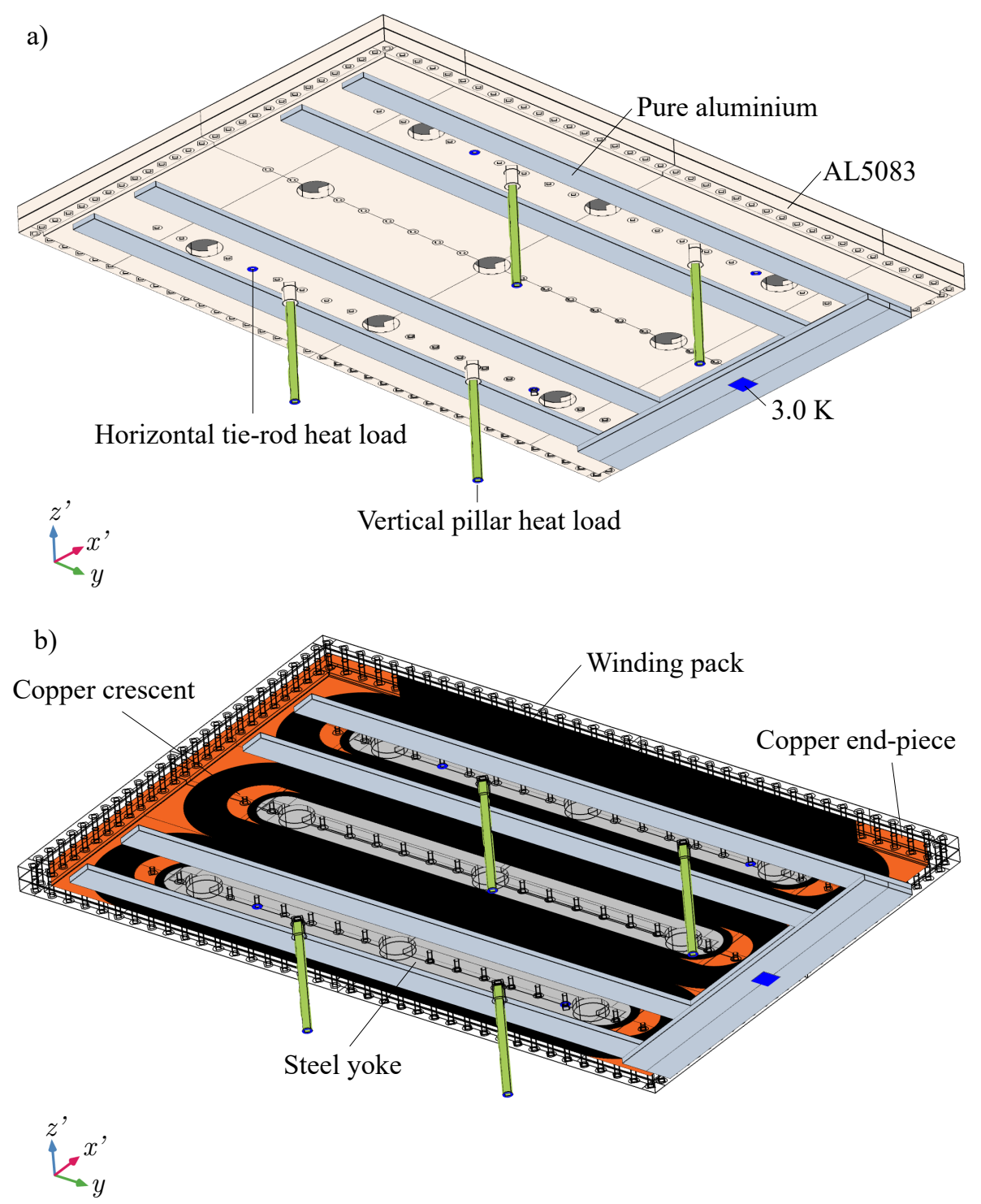

Figure 5.12: a) Geometry used in the thermal model of the cold mass under normal operation. b) Same geometry, with the aluminium alloy cassette hidden for clarity. 
The thermal conductivity perpendicular to the coil turns is estimated by a $2 \mathrm{D}$ simulation in COMSOL using the cell periodicity feature, which was detailed in Section 4.3 on the winding pack's mechanical properties. The resulting effective thermal conductivity at $3.0 \mathrm{~K}$ is $0.15 \mathrm{~W} / \mathrm{m} / \mathrm{K}$. This is larger than the $0.07 \mathrm{~W} / \mathrm{m} / \mathrm{K}$ predicted by the inverse rule of mixtures. The reason behind the difference is that the inverse rule of mixtures assumes that the heat flow has to go through each component in series, whereas in reality the path is partly parallel as well. The value obtained from the simulation was chosen for further calculations.

The thermal conductivity of copper was set to $273 \mathrm{~W} / \mathrm{m} / \mathrm{K}$ in the calculation of the transverse conductivity, corresponding to a magnetic field of $2.5 \mathrm{~T}$ and 3.0 K [93]. This $2.5 \mathrm{~T}$ is the mean magnetic field in the winding pack. Thus unlike the longitudinal thermal conductivity, the perpendicular components do not vary with spatial coordinates in the simulation. The results in the perpendicular direction do not change significantly when the magnetic field is changed, as the effective thermal conductivity is dominated largely by the epoxy. The orthotropic material properties of the winding pack are applied via a curvilinear coordinate system.

The thermal conductivity from the high-purity aluminium heat drains takes into account the magnetic field profile, RRR and temperature and is taken from CryoComp [94].

Table 5.2: Thermal conductivity of winding pack components at $3.0 \mathrm{~K}$ for copper and $4.2 \mathrm{~K}$ for the other materials.

\begin{tabular}{ll} 
Material & Thermal conductivity $[\mathrm{W} / \mathrm{m} / \mathrm{K}]$ \\
\hline Copper RRR 100 & 273 at 2.5 T [93] \\
Stycast 2850FT & $0.05[95]$ \\
Formvar & $0.011[96]$ \\
NbTi & $0.17[38]$
\end{tabular}

The applied boundary loads in the simulation are:

- a radiative heat load of $53 \mathrm{~mW}$. This load is distributed over all external surfaces;

- ramp loss in the winding packs. A value of $0.1 \mathrm{~W}$ is set as a body load on the three coils. This load represents the ramping of the magnet;

- a boundary load of $110 \mathrm{~mW}$ is applied representing the in-leak via the vertical pillars; 
- similarly a load of $35 \mathrm{~mW}$ represents the tie rods.

The connection between the coils and the top aluminium plate is simulated using a $2 \mathrm{~mm}$ thick layer of G11, with thermal conductivity of $0.072 \mathrm{~W} / \mathrm{m} / \mathrm{K}$ [42]. This represents the G11 side-plate of the coils. Likewise the bottom aluminium plate and the coils make contact via a $0.5 \mathrm{~mm}$ thick G11 plate. The high-purity aluminium heat-drain bars make contact with the aluminium alloy bottom plate and with each other via a Stycast layer of $100 \mu \mathrm{m}$ thickness. The same applies to the connection between the coils themselves and between the sides of the coils and the cassette.

The results from the simulation are shown in Figure 5.13. The maximum temperature in the winding pack is $3.07 \mathrm{~K}$ and is located near the anchoring areas of the pillar structure. Because of the geometry of the high-purity aluminium bars and because of the anisotropic thermal conductivity of the winding pack, the temperature distribution of the coils is rather uniform along the conductor and shows variation in the perpendicular directions. Because the gradient across the cold mass is small, it was decided that adjusting the winding pack thermal properties to take into account the fact that the thermal conductivity of Stycast, Formvar and $\mathrm{NbTi}$ is lower at $3.0 \mathrm{~K}$ than at $4.2 \mathrm{~K}$ is not necessary.

The coils are designed for stable operation from a superconducting point of view, at a temperature of no more than $4.5 \mathrm{~K}$. This excludes a $2 \mathrm{~K}$ temperature margin, as discussed in Section 3.1.2. The calculated $3.07 \mathrm{~K}$ maximum is thus below the demand put on the thermal design by the electromagnetic design.

So far it was assumed that the heat load on the second stage is $0.36 \mathrm{~W}$, as calculated in Section 5.2.4, and that the heat transferred from the cold mass to the second stage is $0.29 \mathrm{~W}$, with a cold head temperature of $2.92 \mathrm{~K}$. We can scale the heat loads on the second stage and on the cold mass to get an idea of the margin in the thermal design. It is assumed that the first stage and radiation shield stay at the same temperature as calculated before.

The cold head is able to maintain a $4.2 \mathrm{~K}$ temperature with a $1.5 \mathrm{~W}$ load [52]. If the calculated heat load is scaled up to $1.5 \mathrm{~W}-$ a factor 4.2 increase- $1.2 \mathrm{~W}$ flows from the cold mass to the cold head and $0.3 \mathrm{~W}$ from the current leads to the second stage.

At temperatures around $4 \mathrm{~K}$ the thermal contact resistance decreases with increasing temperature and thermal conductivity increases with temperature [42]. Thus as a pessimistic estimate it can be assumed that the thermal gradient between cold mass and cold head can be scaled linearly by the relative increase of the heat load. This yields a hot-spot temperature of the coils of $4.49 \mathrm{~K}$, which is near to the maximum allowed temperature of $4.5 \mathrm{~K}$.

One could use the margin in the thermal design to ramp the magnet at a higher 


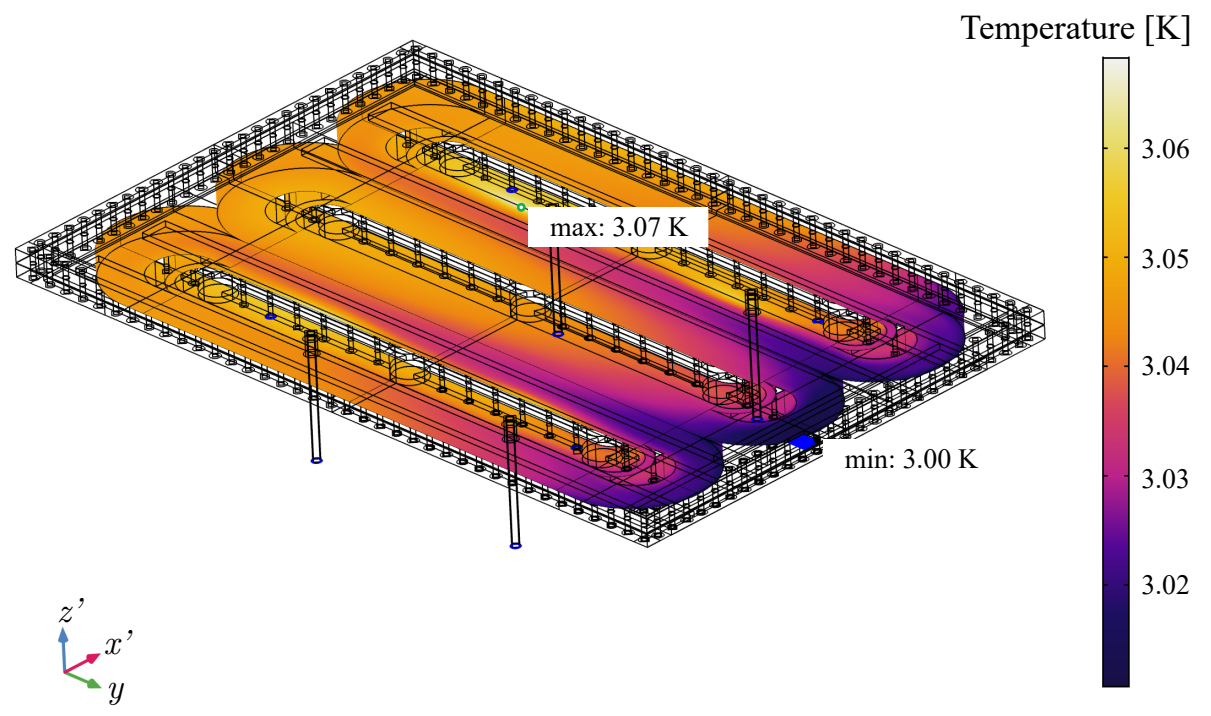

Figure 5.13: Temperature distribution in coils calculated in COMSOL. Heat loads are applied as detailed in Table 5.1, representing normal operating conditions. The coldest spot in the cold mass is fixed to $3.0 \mathrm{~K}$ and the heat loads are applied as detailed in the main text. The warmest spot in the winding pack is less than $0.1 \mathrm{~K}$ above the coldest point.

rate than the $15 \mathrm{~h}$ used in the calculations. Based on Figure 5.6, a 1.5 hour ramp seems possible with a maximum winding pack temperature of $4.5 \mathrm{~K}$.

So it can be concluded that, from a point of view of thermal budget of the cold mass, a margin of around four is present during a ramp of $15 \mathrm{~h}$ and that a quicker ramp is possible from a cooling point of view.

What is not included here is the effect of a possible electrical short in one of the coils. The effect of this is detailed in Section 6.4. It will be shown that the effect of a point load can have a large influence on the hot-spot temperature of the winding pack.

\subsubsection{Cool-down time}

As the coils need to be cooled from room temperature to operating temperatures by the cryocooler via a link, it is important that this link has a high thermal conductivity over a wide range of temperatures. Otherwise, the cold head of the cooler could already cool down to a low temperature itself, while the coils are still much warmer. The result is that the cooling power is decreased. For this reason 
a helium-based heat-pipe is not considered, as the operating range is too narrow [97]. Instead copper and aluminium links as discussed in the previous section are relied upon to transfer heat.

The lumped thermal model introduced in Section 5.2.4 is used to calculate the temperature of the cold mass as a function of time. The mass of components in the calculation is given in Table 5.3. The specific heat as a function of temperature is based on data between $4 \mathrm{~K}$ and $300 \mathrm{~K}$ from [42]. Extrapolation below $4 \mathrm{~K}$ is set to linear ${ }^{4}$.

Table 5.3: Mass of major components in the system.

\begin{tabular}{lll} 
Section & Material & Mass $[\mathrm{kg}]$ \\
\hline Cold mass & Copper & 202 \\
& Steel & 92 \\
& Aluminium & 137 \\
& Stycast 2850FT & 33 \\
& Niobium & 52 \\
Radiation shield & Titanium & 27 \\
& Copper & 27 \\
First stage cryocooler & Aluminium & 55 \\
Second stage cryocooler & Copper & 4.7 \\
& Copper & 3.2
\end{tabular}

The result is shown in Figure 5.14. The first stage of the cooler and the radiation shield cool down in 5 days. After this, there is a small downwards trend. At first, the cold mass is warmer than the shield, and this causes radiation and conduction between the two, the magnitude of which reduces over time.

The cold mass takes slightly less than 16 days to reach a stable temperature of $2.8 \mathrm{~K}$. Here the reader is reminded that the cooling power between $150 \mathrm{~K}$ and room temperature is given a pessimistic value, as introduced in Section 5.2.4. As a rule of thumb, this cryocooler takes around 1 hour to cool-down $1.5 \mathrm{~kg}$ of copper from room temperature to around $4 \mathrm{~K}$ [98]. This would yield a cool-down time of 15 days if the entire cold-mass were copper, close to the obtained results.

\footnotetext{
${ }^{4}$ The linear relation between heat capacity and temperature only holds for metals. As $94 \%$ of the weight of the cold mass is made up from metals, this relation is assumed to hold for the cold mass as a whole.
} 


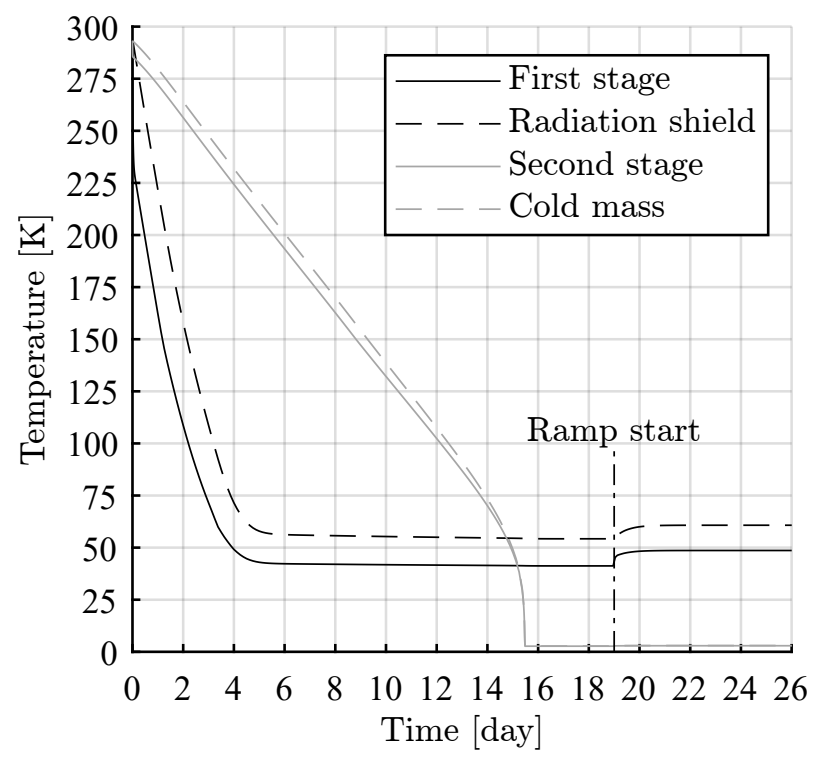

Figure 5.14: Cool-down temperature as a function of time calculated using the lumped thermal model introduced in Section 5.2.4.

The temperature difference between the second stage and the cold mass stays small over the cool-down period. Thus the thermal link is found to be adequate.

In the simulation, the magnet is ramped up to $300 \mathrm{~A}$ in 24 hours, starting at 19 days after the cryocooler is turned on. The major effect of the ramp on the temperature profile is in an increase of the first stage temperature due to the Joule heating in the copper sections of the current leads.

As explained in the next section, the cold mass is expected to warm up to around $100 \mathrm{~K}$ after a quench. It can be seen from Figure 5.14 that it would take the system around 4 days to cool down and recover after a quench. 


\subsection{Electrical circuit}

current leads and protection circuit.

\subsubsection{Self-inductance and stored energy}

The magnet has a total self-inductance of $16.4 \mathrm{H}$. This is built up from the selfinductance of each coil $L_{i}$ and their mutual inductances $M_{i j}$. The values are listed in the matrix in Eq. 5.3.

$$
\left[\begin{array}{ccc}
L_{1} & M_{12} & M_{13} \\
\text { sym } & L_{2} & M_{23} \\
\text { sym } & \text { sym } & L_{3}
\end{array}\right]=\left[\begin{array}{ccc}
4.64 & 0.68 & -0.10 \\
\text { sym } & 4.64 & 0.68 \\
\text { sym } & \text { sym } & 4.64
\end{array}\right] \mathrm{H} .
$$

Here coil 2 is the middle coil. With the operating current of the magnet of $300 \mathrm{~A}$, this leads to a stored magnetic energy ${ }^{5}$ of $0.74 \mathrm{MJ}$.

Safely depositing this energy in a short time is required in three different scenarios: (1) a quench in the magnet, (2) a quench in the current leads and (3) an external emergency situation requiring a fast ramp-down of the magnetic field.

The mass of one winding pack is at $85.8 \mathrm{~kg}$. This means that if all the stored magnetic energy were to be deposited in a single winding pack, the average heat supplied would be $8.7 \mathrm{~kJ} / \mathrm{kg}$. This would heat the winding pack to around $107 \mathrm{~K}$, whereas a maximum temperature of $150 \mathrm{~K}$ is generally accepted [38]. This $107 \mathrm{~K}$ is calculated using temperature dependent heat capacities from [42]. The density of the winding pack is estimated using the rule of mixtures based on data from [42] and is set to $6132 \mathrm{~kg} / \mathrm{m}^{3}$.

In reality a quenching coil would likely transfer enough energy to a neighbouring coil, causing it to quench as well and distributing the stored energy more evenly.

Thus it seems plausible to use a quench protection method where the stored magnetic energy is mainly dissipated in the coils. This is a common quench protection system for low-field superconducting magnets [38, 39]. It has the benefits of being passive and, when diodes are placed parallel to the coils, of not creating high external voltages. In this section first the adopted electrical scheme is introduced, followed by estimates of hot-spot temperatures in coils and the current leads, ramp-down time, voltages and dynamic mechanical forces.

\footnotetext{
${ }^{5}$ The energy associated with the magnetization of the ferrofluid is neglected.
} 


\subsubsection{MDS magnet's electrical circuit}

The electrical circuit of the MDS magnet is shown in Figure 5.15.

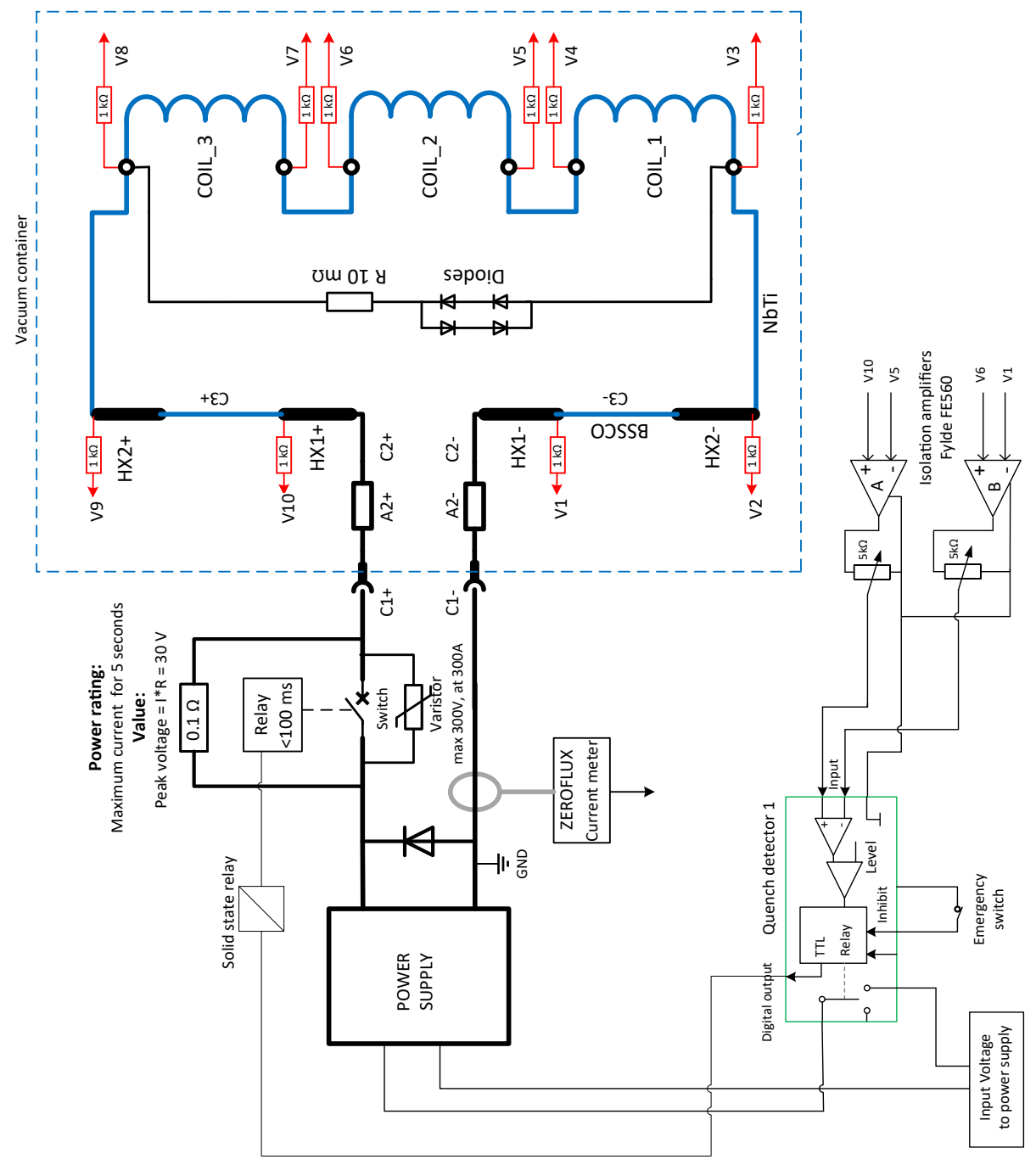

Figure 5.15: MDS magnet's electrical circuit.

The three coils are connected in series. During normal operation the current flows from the power supply through a closed switch, vacuum feed-through $(\mathrm{C} 1+)$, copper current lead (A2+), copper heat exchanger (HX1+) with the first stage of the cryocooler, high-temperature superconductor $(\mathrm{C} 3+)$, heat exchanger with the 
second stage of the cryocooler ( $\mathrm{HX} 2+)$, four parallel $\mathrm{NbTi}$ conductors and finally to the coils.

Connected in parallel to the coils are four diodes. Two of these are connected in forward-, and two in backward direction. These diodes of the Schottky type are located on the cold mass. By placing them in the cold, no additional warm-cold connections are needed. They are rated to handle up to 600 A continuously at room temperature.

The forward voltage of the diodes was measured in a liquid helium bath, and was found to be $2.01 \mathrm{~V}$ at $3.5 \mathrm{~A}$ and $1.60 \mathrm{~V}$ at $300 \mathrm{~A}$. This drop at higher currents is due to heating of the diode. The diodes limit the ramp-rate of the magnet. If it would be desired to ramp at a higher rate, more diodes can be included.

The reason that the diodes are placed parallel to the combination of three coils in series, instead of placing a diode pair across each coil, is that in the selected configuration each coil always carries the same current. In this way a scenario in which one or two coils carry current while another coil is already ramping down is avoided. This keeps the system simpler as it requires less components and connections. Also, it avoids asymmetric forces and local stress.

The voltage tap locations are shown in the schematic. Two pairs are used for quench detection: $\mathrm{V} 5+\mathrm{V} 10$ and $\mathrm{V} 6+\mathrm{V} 1$. This way, a quench originating in the superconducting part of the current leads can be detected as well.

In the case of an emergency, the quench detection system opens the electrical circuit by means of a relay, and the current will flow through the diodes. Also present in series with the diodes is a $10 \mathrm{~m} \Omega$ resistor. This resistor is a steel slab that is glued to the bottom of the cassette. The aim of this resistor is to cause a quench in the coils when the relay has been opened. It may be possible that the dissipation in the diodes is sufficient by itself to cause a quench in this situation, however, the thermal contact between the current-carrying part of the diodes and the cassette is undetermined. Thus the resistor is added. By causing an intentional quench when the relay is opened, a fast ramp-down of the magnet can be achieved in the case of an emergency in the industrial operating environment. The timescale of the ramp-down is around 2 to 3 seconds, as explored later in the chapter.

The current leads consist of copper parts to carry current between room temperature and the first stage of the cryocooler. These are represented in the diagram as A2 and are heat-sunk at HX1. From the first stage of the cryocooler to the second stage three BSCCO-2223 tapes (Sumitomo type G) per lead are present with specifics as detailed in Section 5.2.

If a single one of these tapes transitions to the normal state, the other two tapes in the lead are able to carry the transport current while remaining superconductive as there is enough margin, see Section 5.2. A possible failure scenario causing all three tapes in a lead to quench is a temperature rise of the first stage of the 
cryocooler. In principal this temperature rise can be used as a warning, and the magnet can be ramped down by the power supply [99].

However, it is important to make sure that the BSCCO tapes do not burn out in the case a quench does occur and the metal matrix needs to conduct the transport current for a short period.

\subsubsection{Current lead temperature during fault}

In the case of a quench in the superconducting part of the current leads, the metal matrix need to conduct the current until the switch in the electrical circuit (Figure 5.15 ) is opened. To get an estimate of what time is allowed to detect a quench and open the switch, a 1D FEM calculation is performed in COMSOL. The simulation is a coupled heat transfer- and electrical currents calculation.

The specific heat, thermal conductivity and electrical resistance (at zero external magnetic field) of the tape are taken from the Sumitomo data sheet [88]. The mass density is set to $9.3 \mathrm{~g} / \mathrm{cm}^{3}$, based on an estimated composition of $70 \%$ silver $\left(10.5 \mathrm{~g} / \mathrm{cm}^{3}[42]\right)$ and $30 \%$ BSCCO filaments $\left(6.4 \mathrm{~g} / \mathrm{cm}^{3}[100]\right)$.

The tapes are considered to be thermally isolated from their G11 backbone and it is assumed that no heat can leak towards the cryocooler. The ratio between the thickness and length of the tape+G11 combination is roughly the same as the ratio between the thermal conductivity in the longitudinal direction (dominated by the silver-gold matrix) and the thermal conductivity in the perpendicular direction (dominated by the G11). The model is thus pessimistic.

The calculated temperature profile along the tapes is shown in Figure 5.16 for different times after the tapes have been driven normal. At the start of the simulation, one end of the tape is at $4.5 \mathrm{~K}$ and the other end at $77 \mathrm{~K}$. The maximum temperature after $0.6 \mathrm{~s}$ is around $200 \mathrm{~K}$. This is considered to be the maximum allowed temperature. Taking into account a switching time of the relay of $0.1 \mathrm{~s}$, this leaves $0.5 \mathrm{~s}$ to detect (by measuring the potential over the voltage taps) and react on a quench signal. This is thought to be sufficient time. Note that in reality heat transfer from the tapes to the cryocooler and from the tapes to the G11 backbone will reduce the hot-spot temperature compared to the calculated values. As mentioned in Section 5.2.3, the current leads' heat exchangers contain a large fraction of copper, which is beneficial in distributing the Joule heating in the BSCCO tapes away from the tapes themselves.

\subsubsection{Simulation of a quenching coil}

A straightforward calculation as presented in Section 5.3.1 resulted in an estimated temperature of a thermally isolated coil during a quench of $107 \mathrm{~K}$. Here 


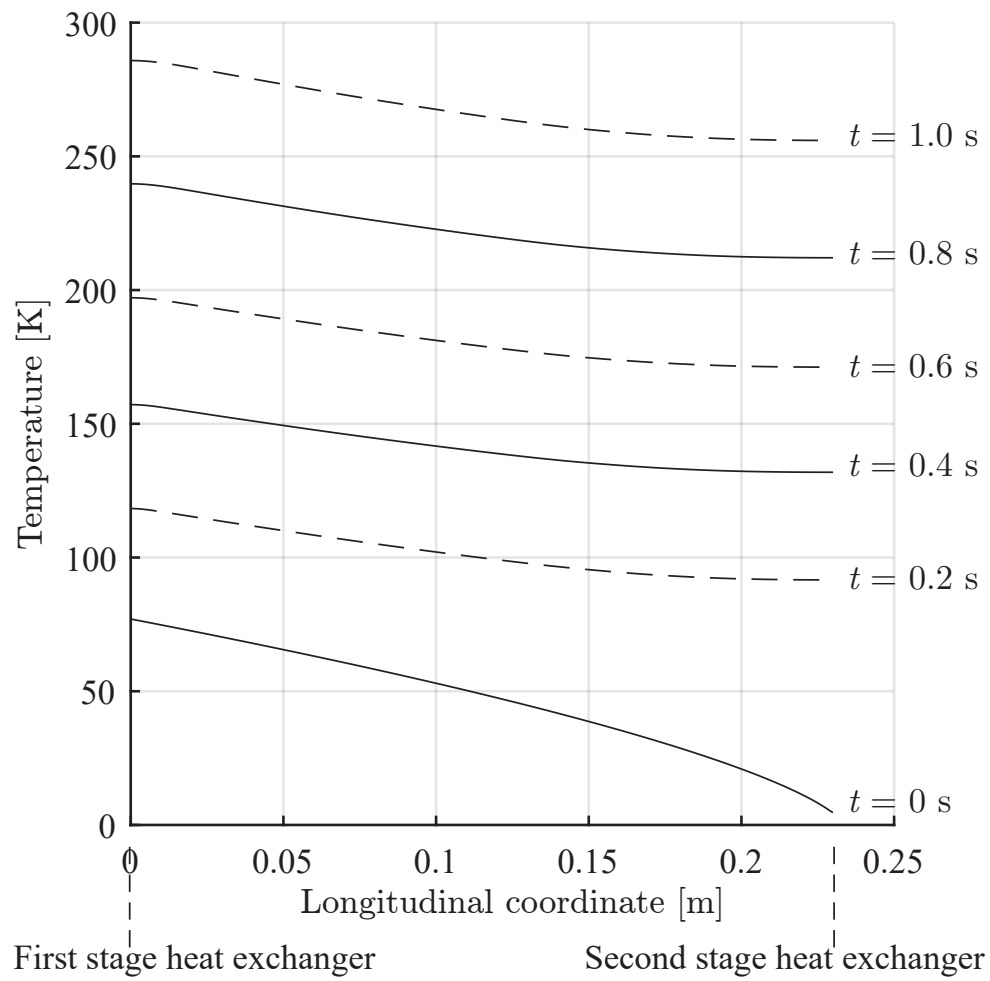

Figure 5.16: Computed temperature profile in the BSCCO current leads as a function of time for a fault scenario in which the silver-gold matrix carries the full operational current starting at $t=0 \mathrm{~s}$.

it was assumed that the dissipated energy is distributed uniformly. However, as was calculated in Section 5.2.5, the effective thermal conductivity at $4 \mathrm{~K}$ of the winding pack perpendicular to the turns is a factor thousand less than along the conductor. Thus it is of interest to find out how this anisotropy influences the hot-spot temperature during a quench.

Quench simulations were performed in COMSOL to determine:

- the maximum temperature in the winding pack during a quench;

- the maximum voltage within the winding pack;

- the feasibility of quenching the coil on purpose in the case of a trigger by the emergency switch to enforce a rapid ramp-down;

- the dynamic force on the high-purity aluminium heat drains, due to induced 
currents. This is of interest because it allows an estimate whether simply glueing the bars to the cassette provides sufficient strength.

Two different simulations will be presented. The first concerns the adiabatic quench behaviour, and provides estimates of peak temperature and voltage. The second simulation allows for heat exchange with the cold mass and induction of eddy currents, leading to a more realistic quench evolution.

\subsubsection{Adiabatic winding pack}

First the focus is on the adiabatic quench simulation. Here adiabatic refers to the winding pack being considered as thermally isolated from the surroundings. Heat transfer within the winding pack, and thus between adjacent coil turns is possible and the winding pack is considered to be a homogeneous composite with orthotropic material properties. A schematic showing the interaction between the electrical circuit-, heat transfer- and magnetic field calculations can be found in Figure 5.17.

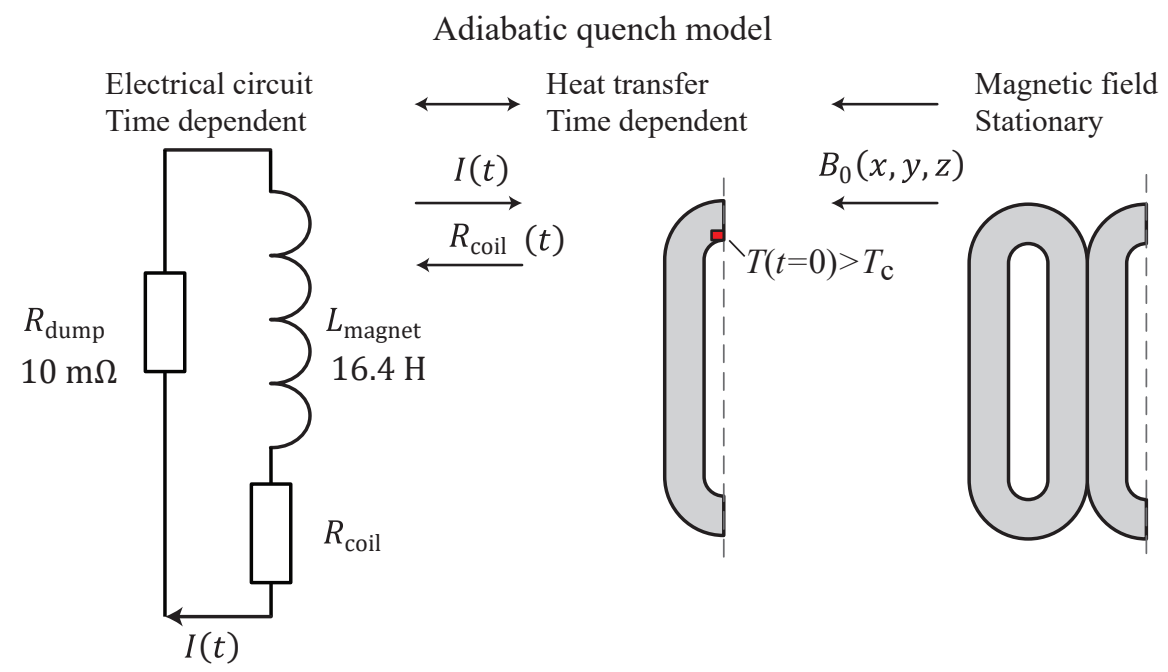

Figure 5.17: Overview of interaction between different simulation components for the adiabatic quench model. At each time-step the electrical network receives the resistance of the quenching coil in order to determine the current. This current is used in the thermal model to determine the local ohmic dissipation. To account for magneto-resistance, the static magnetic field profile is scaled with the current via Eq. 5.6. 
This simulation was performed as it was thought to produce the highest hotspot temperature, and as it could act as a starting point for more detailed calculations.

The electrical circuit is represented by a lumped system consisting of a $10 \mathrm{~m} \Omega$ dump resistor $R_{\text {dump }}$ in series with the coils. The self-inductance of each coil and their mutual inductances, as listed in Section 5.3.1, are lumped into one selfinductance $L_{\text {magnet }}$. This is correct since each coil carries the same current.

The resistance of the coil $R_{\text {coil }}$-equivalent to the resistance of the normal zone- is calculated at each time step using the temperature- and magnetic field dependent average resistivity of the conductor, $\bar{\rho}(T, B)$ via

$$
R_{\mathrm{coil}}=\frac{\bar{\rho}(T, B)}{A_{\mathrm{cu}}} l_{\mathrm{wire}}
$$

where $A_{\mathrm{cu}}$ is the cross-sectional area of the copper matrix in the wire of $0.84 \mathrm{~mm}^{2}$ and $l_{\text {wire }}$ is the length of conductor in the winding pack of $6.3 \mathrm{~km}$. The average resistivity is calculated by

$$
\bar{\rho}(T, B)=\frac{1}{V_{\text {windingpack }}} \iiint_{V_{\text {windingpack }}} \theta(x, y, z, T) \rho(x, y, z, T, B) \mathrm{d} V,
$$

where $V_{\text {windingpack }}$ is the volume of the winding pack and $\rho$ is the resistivity of copper with RRR 100, taken from [93]. $T$ and $B$ both vary with spatial coordinates. $\theta$ is a smoothed Heaviside step function that provides a simplified representation of the superconducting-normal transition. It is zero below $6 \mathrm{~K}$ and one above $7 \mathrm{~K}$. Thus regions below $6 \mathrm{~K}$ do not contribute to the resistivity. As the quench propagation itself is not a major focus point of the simulation, this simplification of the actual magnetic field dependent superconducting properties is thought to be sufficient.

The magnetic field dependence of the copper resistivity is taken into account by scaling the magnetic field of a stationary magnetic field calculation $B_{0}$ with the current $I(t)$ relative to the starting current $I_{0}$ :

$$
B(x, y, z, t)=B_{0}(x, y, z) \frac{I(t)}{I_{0}} .
$$

The calculated resistivity is used in combination with the current derived from the lumped electrical network to determine the local dissipation $P$, in $\mathrm{W} / \mathrm{m}^{3}$, in the winding pack

$$
P(x, y, z, T, B)=\theta(x, y, z, T) \rho(x, y, z, T, B) \frac{I^{2}(t)}{A_{\mathrm{cu}}^{2}} .
$$


The specific heat of the winding pack as a function of temperature is calculated using the rule of mixtures using data from [42]. For NbTi the heat capacity at zero magnetic field is used. As there is only a difference in specific heat at low temperatures $(<10 \mathrm{~K})$, and the specific heat is small at those temperatures, this is not thought to influence the results significantly.

The orthotropic thermal conductivity in the winding pack is taken into account by introducing a curvilinear coordinate system. In the longitudinal direction the thermal conductivity is set to $38.8 \%$ of that of RRR 100 copper. This corresponds to the copper volume fraction in the winding pack volume. The thermal conductivity perpendicular to the windings is set to $0.15 \mathrm{~W} / \mathrm{m} / \mathrm{K}$ at $4.5 \mathrm{~K}$ as determined in Section 5.2.5 and is given the temperature dependence of thermal conductivity of Stycast 2850FT [101].

The heat loads during normal operation are omitted from the simulation, as they are a factor $10^{5}$ smaller than the dissipation inside the winding pack. The diodes are also not included in the model, as their highly non-linear characteristics required unacceptable calculation times. The effect of this on the simulation results is small, as the diodes would dissipate only a fraction of the total dissipated energy.

The initiation of the quench is modelled by setting the initial temperature of a small section of one of the racetrack's inner heads to $7 \mathrm{~K}$, as indicated in Figure 5.18. It can be seen in the figure that the whole coil is in the normal state at $0.04 \mathrm{~s}$.

The maximum and minimum temperatures in the winding pack are presented in Figure 5.19a as a function of time. The maximum is $113 \mathrm{~K}$. The average temperature in the winding pack is $97 \mathrm{~K}$. This is lower than the estimated $107 \mathrm{~K}$ in the case of uniform heating, and is the result of the increase of heat capacity with temperature. Also, part of the stored energy is dissipated in the dump resistor instead of in the winding pack.

It can be observed from Figure 5.19a that the whole winding pack is in the normal state after only $0.03 \mathrm{~s}$. This corresponds to a longitudinal quench propagation velocity of around $46 \mathrm{~m} / \mathrm{s}$, which is a realistic value for $\mathrm{NbTi} / \mathrm{Cu}$ conductor [39]. From this it was concluded that the simplification of the superconductingto-normal transition is sufficient for the intended aims of the simulation.

The temperature in the coldest spot in the winding pack stays below $88 \mathrm{~K}$. This cold spot is located in the middle of the winding pack, where the magnetic field is relatively low. In the high magnetic field region, the magneto-resistance of the copper matrix causes this region to heat up more than the low-field region. This gives the high-field region a head start in reaching the higher temperatures in which magneto-resistance has a smaller effect, but as the resistivity increases with temperature this region maintains the higher temperature as long as there 


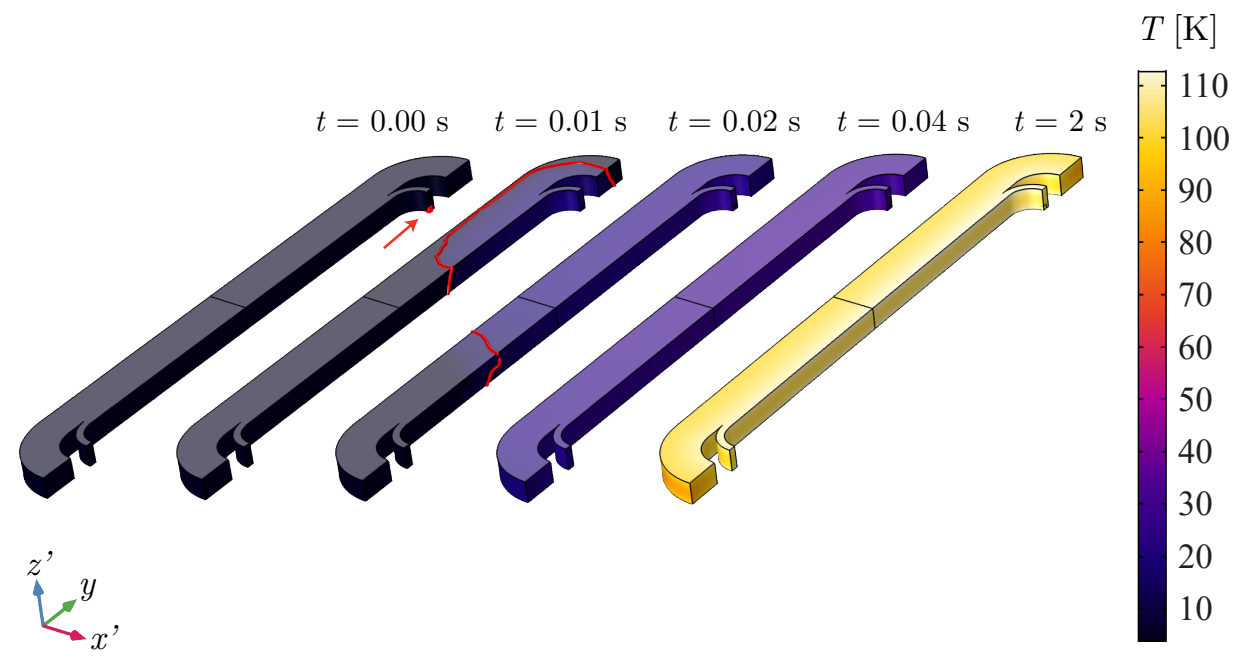

Figure 5.18: Temperature profile of quenching coil at selected times. At $t=0 a$ small normal zone is present, indicated by the arrow. The zone expands over time and ohmic heating causes the temperature of the coil to increase. The transition between the normal-and superconducting state is indicated by a red border. The winding pack is thermally isolated from the rest of the cold mass.

is energy available for dissipation. Due to the low thermal conductivity of the winding pack perpendicular to the windings, the temperature gradient only slowly becomes smaller after the maximum has been reached.

The computed resistance is shown in Figure 5.19b. A discontinuity is present at $0.03 \mathrm{~s}$, due to the normal zone having occupied the entire winding pack at this point. The resistance climbs up to a value of $29 \Omega$ and then stabilizes.

This resistance is much higher than that of the $10 \mathrm{~m} \Omega$ dump resistor, and thus the current, presented in Figure 5.19c, starts to decay significantly only when the resistance of the coil starts to increase. After three seconds the current is below $5 \mathrm{~A}$.

The maximum voltage occurs inside the winding pack, where the inductive and resistive voltages are opposed to each other. This internal voltage is approximated using [39]

$$
V_{\text {internal }}(t)=I(t) R_{\text {coil }}(t)\left(1-\frac{L_{\text {normal }}(t)}{L_{\text {magnet }}}\right),
$$

where $L_{\text {magnet }}$ is the total inductance of the system and $L_{\text {normal }}$ is the inductance 

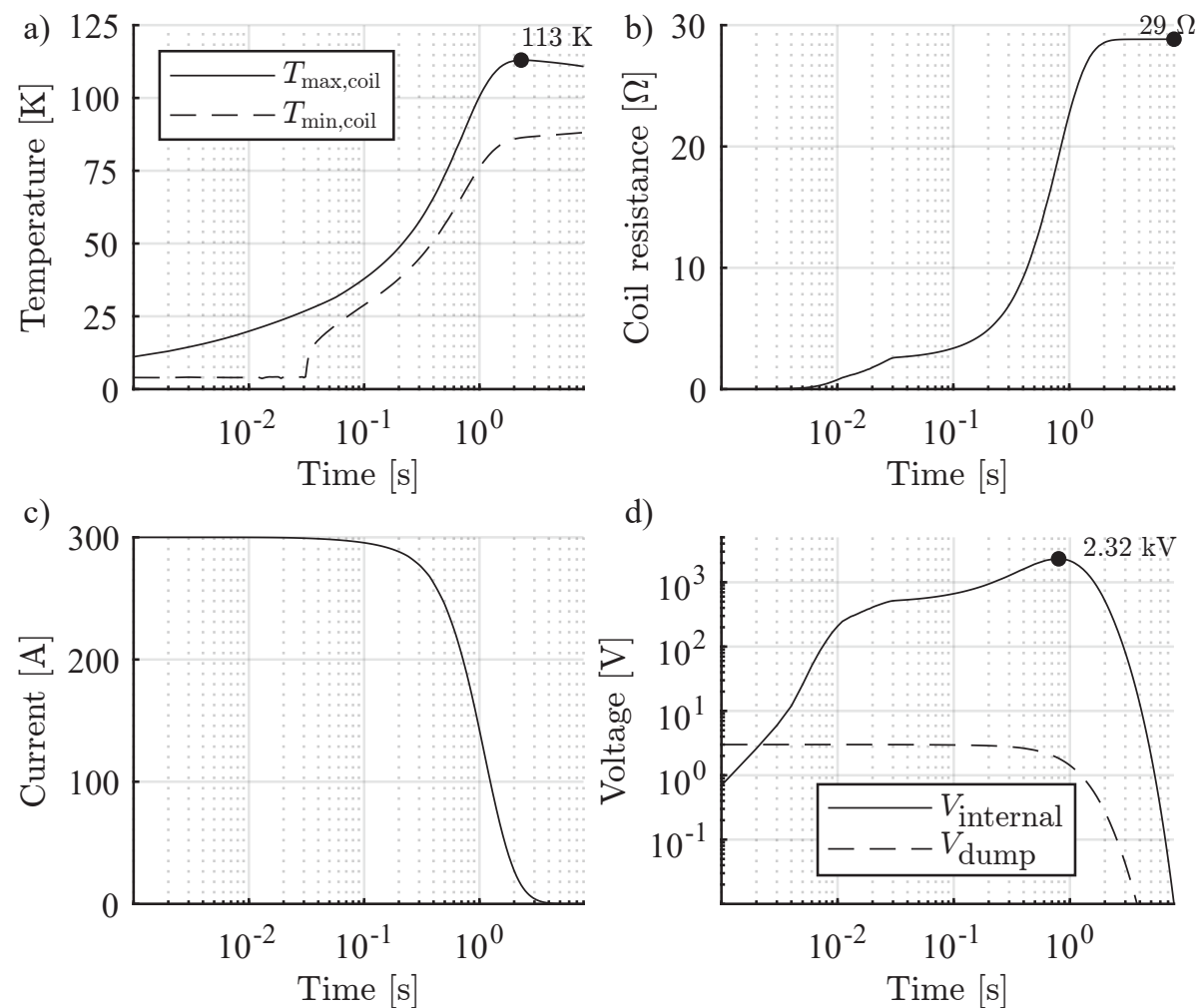

d)
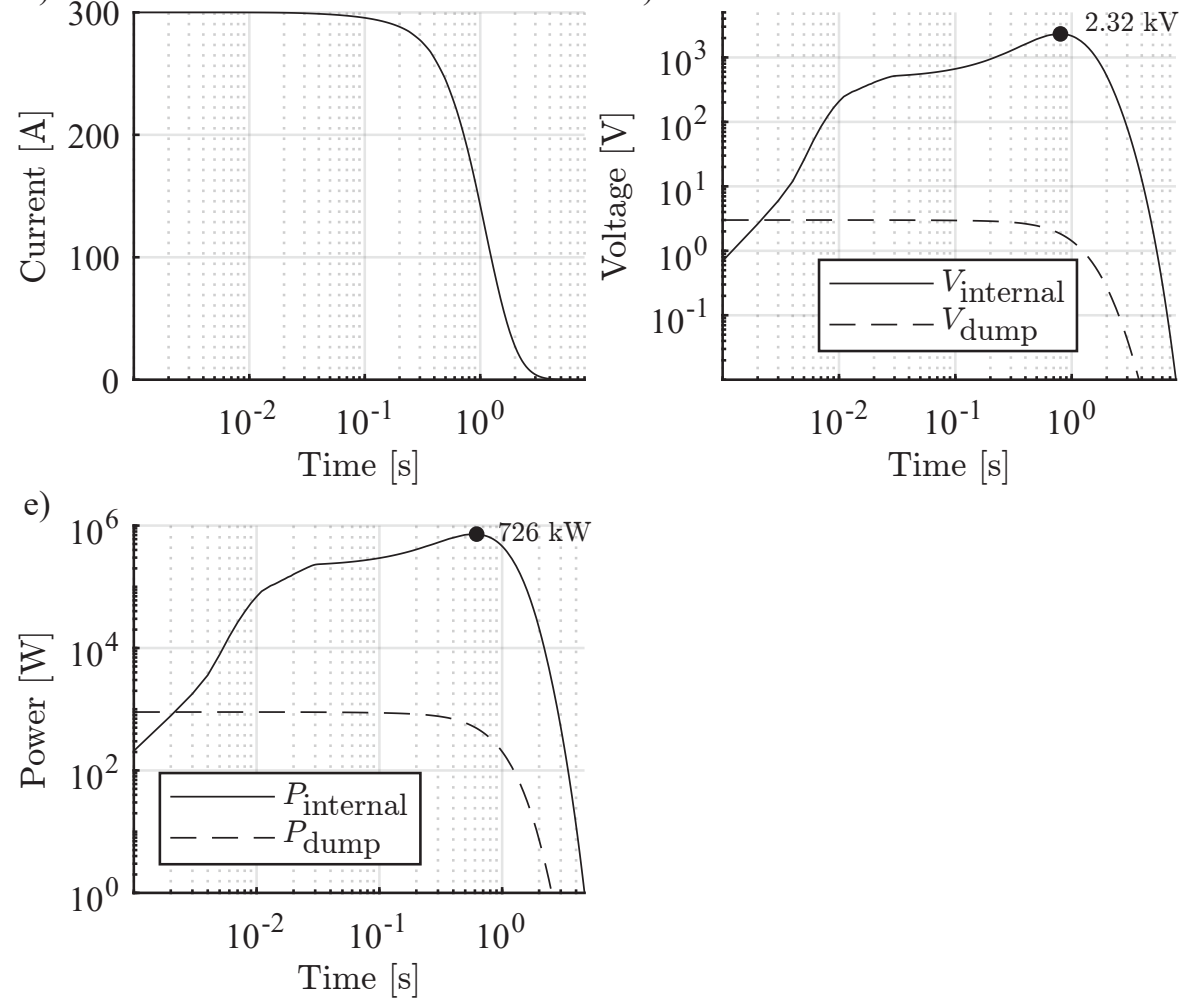

Figure 5.19: Results as a function of time are shown of calculations for a quenching winding pack that is thermally isolated. a) Temperature inside the winding pack. b) Resistance of the coil. c) Current in the circuit. d) Voltage, inside the winding pack and across the dump resistor. $\mathrm{e})$ Dissipation inside the winding pack and in the dump resistor. 
of the normal zone. Assuming that the voltage is not high enough to create an electrical short, $L_{\text {magnet }}$ is time-independent. In the calculation, $L_{\text {normal }}$ has a value between 0 and $L_{\text {magnet }} / 3$, which is a simplification but sufficient for the required accuracy. The maximum calculated internal voltage is around $2.3 \mathrm{kV}$, see Figure 5.19d. In the considered scenario, in which the middle coil quenches but the two coils on each side remain superconducting, this voltage would be present between the current leads of the middle coil. However, as the coils touch their neighbours over a wide contact area, it is likely that a quenching coil will quickly drive its neighbours normal as well, and thus this internal voltage can be kept lower, as $L_{\text {normal }}$ approaches $L_{\text {magnet }}$.

The dissipated power as a function of time is presented in Figure 5.19e. The maximum dissipation inside the winding pack is $726 \mathrm{~kW}$. The dump resistor dissipates $R_{\text {dump }} I^{2}=900 \mathrm{~W}$ initially. This value decreases with time as the current decays. The electrical resistance of the dump resistor is not adjusted for temperature. As the resistor is made out of steel, and steel does not have a large temperature dependent resistance, this is a valid assumption [42]. The diodes, which are part of the protection scheme but not included in this calculation, would each dissipate roughly half that of the dump resistor. The effect of neglecting the diodes on the energy dissipated in the winding pack thus seems small.

By integrating the dissipated power over time, the dissipated energies are obtained: a value of $6.4 \mathrm{~kJ}$ in the dump resistor and $717 \mathrm{~kJ}$ in the winding pack. Together this makes up $97.8 \%$ of the energy initially stored in the magnetic field, of $E=0.5 L I^{2}=0.5 \cdot 16.44 \cdot 300^{2}=740 \mathrm{~kJ}$. At the end of the calculation, the current has decayed below a milliampere, thus no energy remains stored in the system that can explain the difference. Also, the density of the considered data-points in the considered time-range is high (288 points). The $2.2 \%$ difference is thought to be small enough to draw conclusions regarding the thermaland electrical behaviour.

From the adiabatic model a hot-spot temperature of around $113 \mathrm{~K}$ is calculated, which is below the maximum allowable value of $150 \mathrm{~K}$. In reality it is likely that once a coil quenches, the other coils quench as well, if not from conduction of heat from the quenching coil to neighbours, then by ramping losses caused by the rapid change of the operating current (the energy dissipated by hysteresis effects in the superconductor is around $3 \mathrm{~kJ}$, see Section 5.2.2.5). Thus a protection strategy based on dissipation of the stored magnetic energy in the winding pack is feasible for this magnet.

\subsubsection{Thermally connected winding pack}

After the results from the adiabatic simulation, it was decided to perform a more detailed calculation. There are two main differences with the previous calculation. 
One is that the winding pack can exchange heat with the stainless steel yoke, aluminium cassette and copper end-pieces. The cooling of the outer surface of the coil could result in a less uniform heating of the winding pack and thus to a higher hot-spot temperature. The second reason is that the magnetic field calculation is made time-dependent, allowing the generation of induced currents.

An image of the simulation geometry used for the thermal calculations is shown in Figure 5.20. The geometry consists of a simplified version of that used in the stationary heat profile calculation of Section 5.2.5. One point of interest regarding this thermal calculation is to see the required time-scale in the case a heater underneath the cassette is activated with the goal of invoking a quench on purpose, for example when an emergency switch is pressed by the operator. By putting the resistor in series with the diodes, see Figure 5.15, no separate current leads for the heater are required, and the heater is activated automatically by opening the switch in the main current loop.

A second point of interest in this simulation concerns the fact that the aluminium cassette, and especially the copper end-pieces and high-purity aluminium heat drain bars have a much higher thermal conductivity than the winding pack has in the direction perpendicular to the windings. This may have a significant effect on the temperature profile in the winding pack, and thus on the electrical resistance and the time-scale of the ramp-down.

Another reason for performing the simulation is to obtain an estimation of the induced forces in the high-purity aluminium.

The two outside coils are not taken into account in the thermal calculation, as it was thought that the hot-spot would be higher when assuming that only the middle coil quenches. In reality the middle coil would likely quickly transfer enough energy to the outer coils to make these quench as well. Thus the selected scenario can be regarded as a worst-case as far as the hot-spot temperature is concerned. Also, by only considering thermal aspects of a single coil, comparison with the previously discussed adiabatic model can be more easily made.

The second main difference with the adiabatic calculation is that the magnetic field is now calculated at each time step. The geometry used is the same as that for the thermal calculation, with the difference that the outside coils are also included as well as the addition of an air domain.

The interaction between the different physics in the calculation is shown in Figure 5.21. The electrical circuit is kept the same as in the adiabatic quench model. The heat transfer study receives the magnitude of the current from the electrical circuit to calculate the dump resistor dissipation. The current is also used to calculate the magnetic field generated by the coils. The temperature dependent electrical resistivity is calculated by using temperature data from the heat transfer study. The calculated magnetic field is used to take into account magnetic-field- 


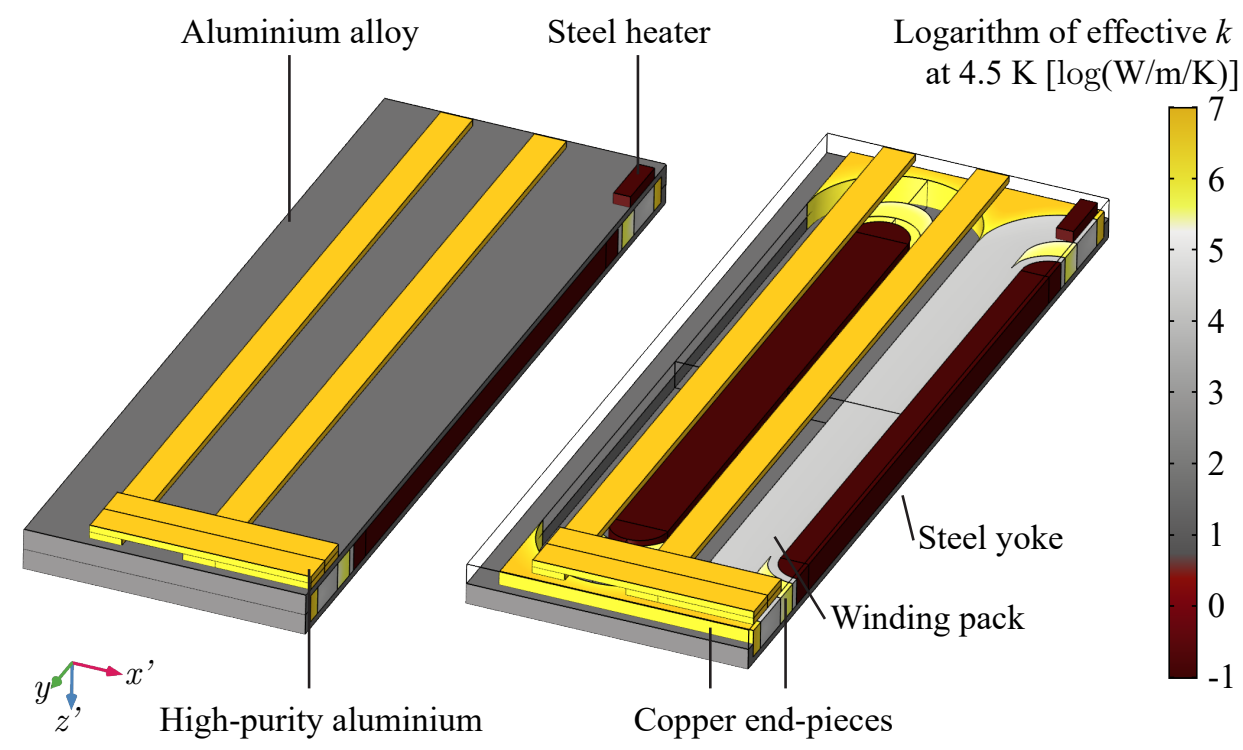

Figure 5.20: Simulation geometry for thermal calculations, consisting of a half of the simplified cold mass, viewed from the bottom. The left image shows the full simulation geometry, and the right image has one of the two parts that make up the aluminium alloy cassette removed to enable a clearer view. One can see the stainless steel yokes and one winding pack. Also shown are copper crescents and end-pieces. Underneath the cassette high-purity aluminium heat drains are present. The dump resistor which acts as a heater to initiate a quench is located on one end of the cold mass. The objects are coloured based on the logarithm of their effective thermal conductivity at $4.5 \mathrm{~K}$ and full operating magnetic field.

dependent thermal conductivity in the heat transfer calculation. The electrical resistance of the coil is calculated by COMSOL's coil module and is given as an input for the electrical circuit. The calculated electrical resistivity of the winding pack as well as the eddy current heating are used in the heat transfer module.

A possible effect of the presence of the aluminium heat-drain bars is that they can act as a coupled secondary coil. The changing magnetic field in the magnet induces currents in the bars which in turn influence the decay of the current in the magnet. The transfer of stored magnetic energy away from the winding pack using a coupled secondary is a well-known quench protection method [39]. In the presented calculation however, the coupling is not made in the electrical circuit. This means that the winding pack and dump resistor together dissipate the energy stored in the magnetic field, but on top of that the energy dissipated by generation 
Non-adiabatic quench model

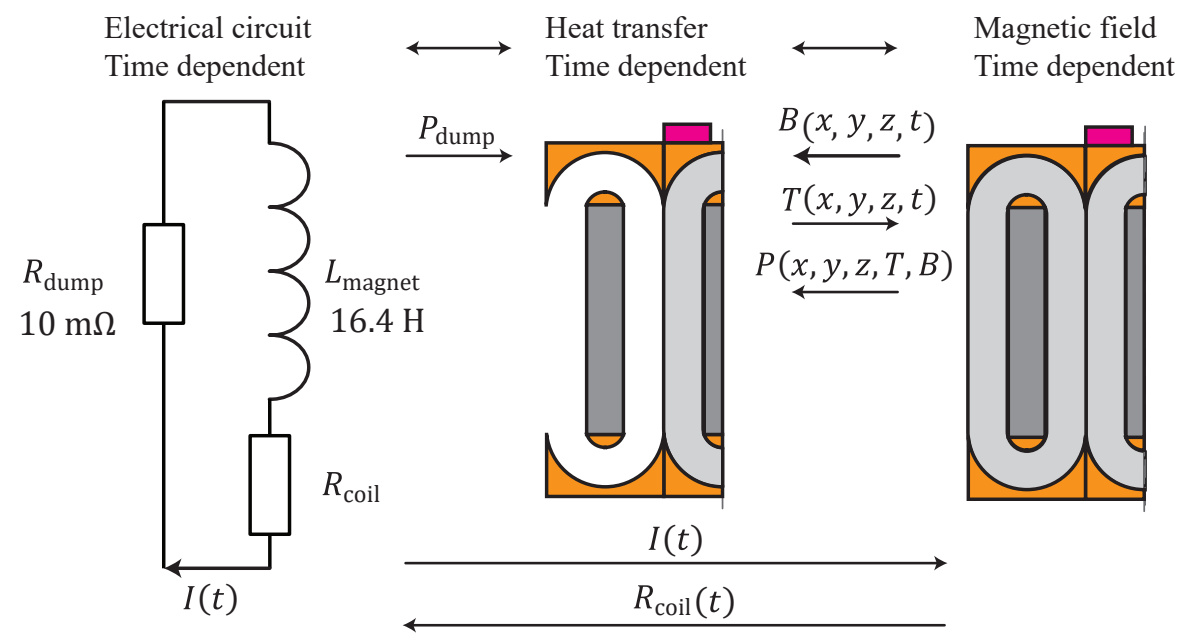

Figure 5.21: Overview of interaction between different simulation components for the quench model of a thermally connected winding pack. Electrical circuit-, heat transfer-and magnetic field calculations exchange information at each time step.

of eddy currents in the bars is added. However, it will be shown that the relative magnitude of this latter contribution is relatively small.

The steel heater is placed below one of the heads of the middle coil. In this way, the simulation geometry can be halved because of symmetry. In reality it is probably better to put the heater below one of the straight sections of the center coil, further away from the copper parts of the coil. In the simulation, the heater starts carrying the operating current after $50 \mathrm{~ms}$. The interfaces between different components are modelled as a $100 \mu \mathrm{m}$ layer of Stycast 2850FT.

The temperature profile of the winding pack at selected time-steps is presented in Figure 5.22. The maximum and minimum temperatures of the winding pack are shown in Figure 5.23a as a function of time. The coil starts to quench at around $90 \mathrm{~ms}, 40 \mathrm{~ms}$ after the heater is powered on, and the whole winding pack is in the normal state after $130 \mathrm{~ms}$.

Compared to the adiabatic winding pack temperature profile, as was presented in Figure 5.19, the maximum temperature is now higher, but still acceptable, with a value of $135 \mathrm{~K}$. The reason behind this increase is that the boundaries of the winding pack are now effectively cooled by the surrounding cold mass, which 


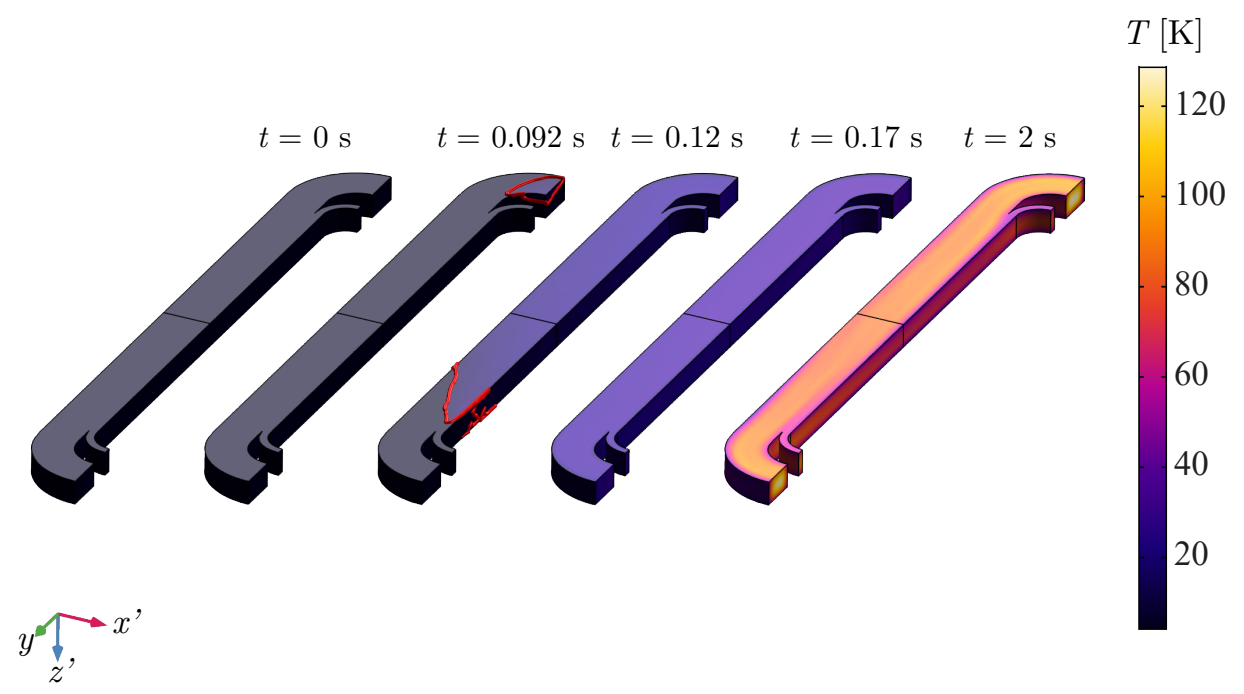

Figure 5.22: Temperature profile of quenching coil at selected times. The quench is initiated by a heater, which is shown in Figure 5.20. The transition between the normal-and superconducting states is indicated by a red border. The coil is viewed from the bottom. Heat transfer with the rest of the cold mass is allowed.

has a relatively good thermal conductivity compared to that of the winding pack perpendicular to the windings. As a result, only the inner bulk of the winding pack dissipates significant heat, allowing the hot-spot temperature to become larger.

The steel heater does not overheat, see Figure 5.23a, thus simply glueing the heater to the aluminium cassette seems a good solution.

As the simulation allows for the induction of eddy currents, it is interesting to see the magnitude of the currents flowing in the high-purity aluminium bars. The current density in the $y$-direction in the winding packs and the bars is presented in Figure 5.24.

Integrating the current density over an $x^{\prime} z^{\prime}$-cross section at the $y$-symmetry plane of the coils yields the ampere-turns versus time, shown in Figure 5.23f. The peak current flowing in the aluminium bars is significant compared to that of the coils (up to 23\%). However, as the electrical resistivity of the high-purity aluminium (RRR 1500) is much lower than that of the copper matrix of the conductor (RRR 100), the relative contribution to the dissipated power is $4 \%$ at maximum, see Figure 5.23e.

The calculated dissipation is $742 \mathrm{~kJ}$ in the winding pack, $24 \mathrm{~kJ}$ in the heatdrain bars, $1 \mathrm{~kJ}$ in the heater and $7 \mathrm{~kJ}$ in the copper end-pieces and crescents. This is $4.6 \%$ higher than the energy initially stored in the magnetic field of $740 \mathrm{~kJ}$ 

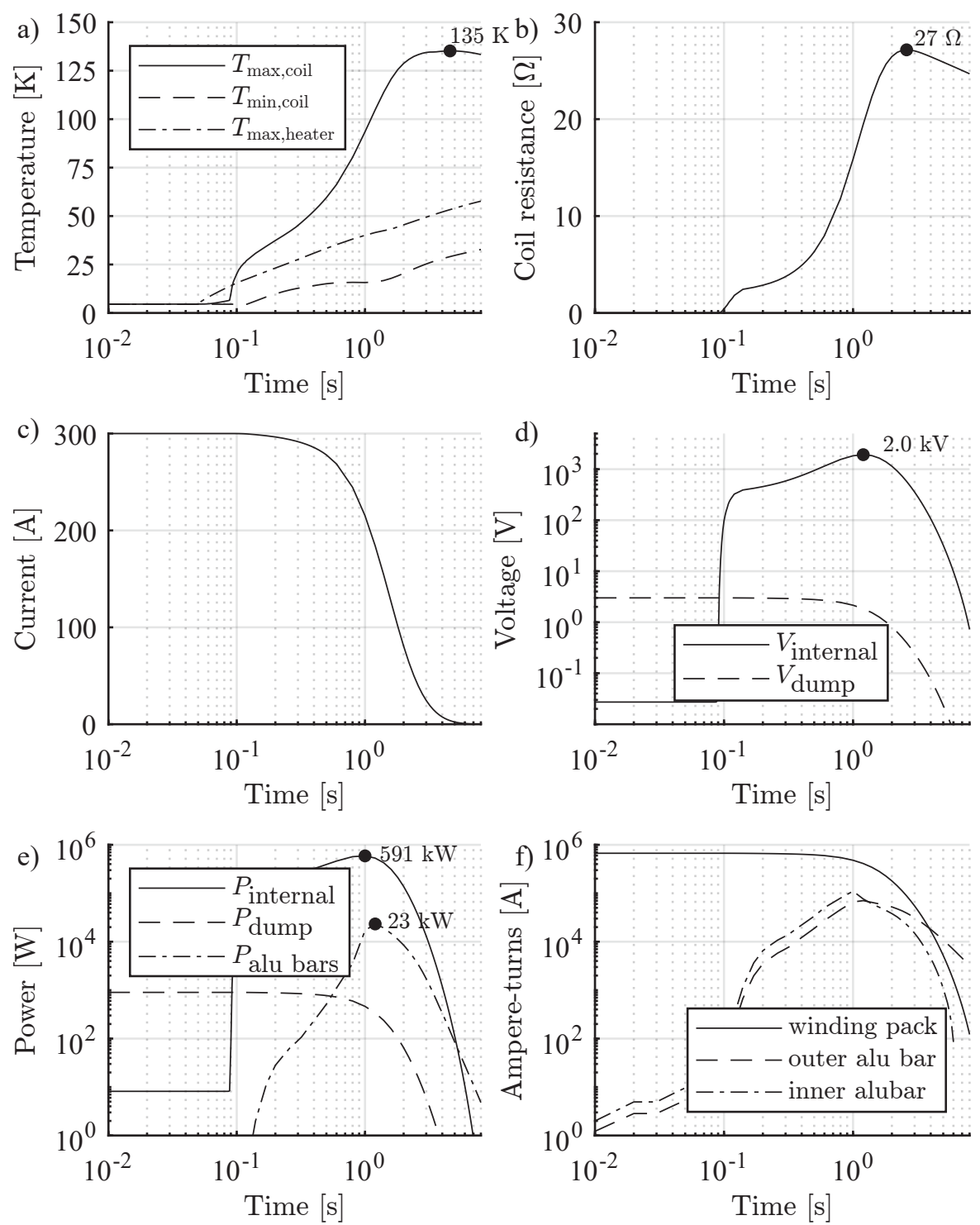

Figure 5.23: Results as a function of time are shown of calculations for a quenching winding pack that is thermally connected to the cold mass. a) Temperature inside the winding pack and heater. $\boldsymbol{b})$ Resistance of the coil. c) Current in the circuit. $\boldsymbol{d})$ Voltage, inside the winding pack and across the dump resistor. $\boldsymbol{e})$ Dissipation inside the winding pack, in the dump resistor, and in the high-purity aluminium heat drains. f) Ampere-turns in the winding pack and the high-purity aluminium bars. 
and is a result of the fact that the eddy currents are not included in the electrical network. As a consequence, the current in the coils decays at a lower rate than if these effects were included, causing extra dissipation in the system. Thus, the results are interpreted as a worst-case scenario.

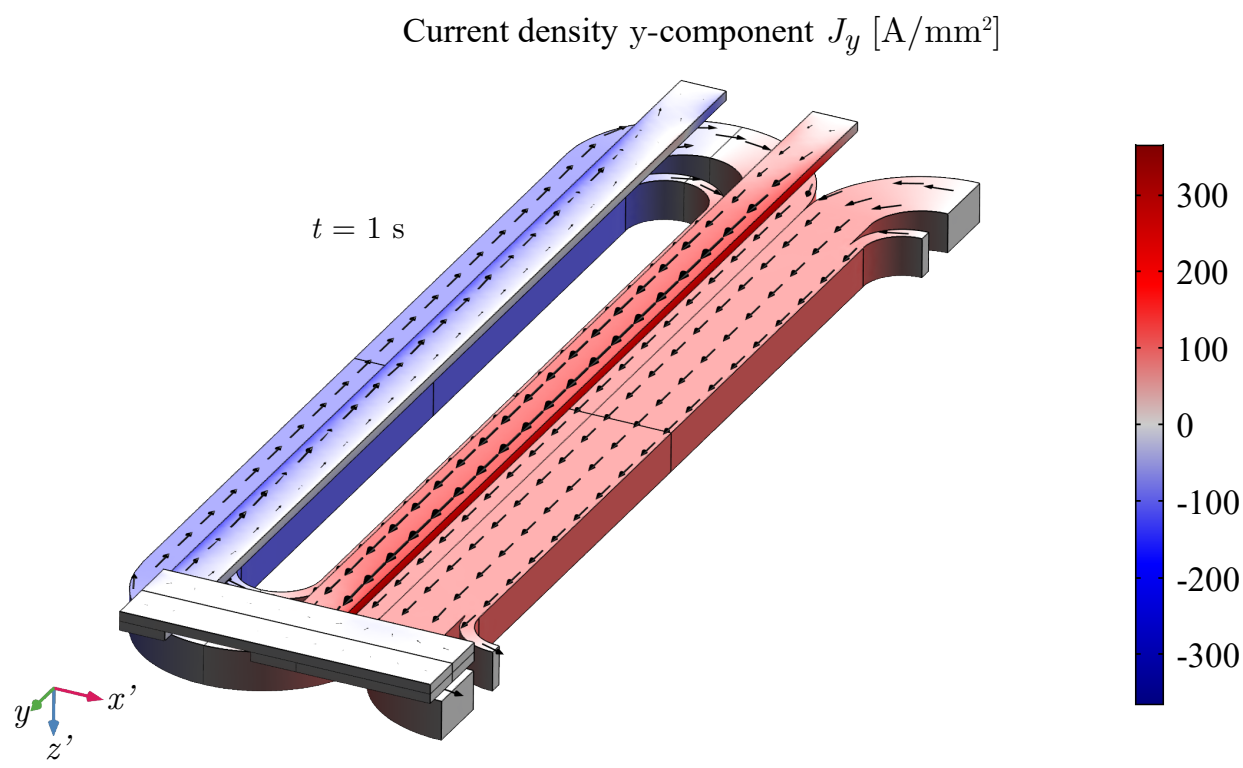

Figure 5.24: Current density in y-direction after $1 \mathrm{~s}$. The current density in the high-purity aluminium heat drains has a similar magnitude to that of the winding pack. The direction of the current density vector is shown by black arrows.

From the induced current density in the high-purity aluminium heat drain bars and the magnetic field, the dynamic Lorentz force on these bars can be calculated by integration. The maximum horizontal force on the outer heat-drain bar is $105 \mathrm{kN}$, pointing outwards. For the inner bar the maximum horizontal force has the same magnitude, directed inwards. This results in a shear stress of $2 \mathrm{MPa}$ on the epoxy connection between the bars and the cold mass, which is acceptable.

From the calculations regarding the quenching of a winding pack thermally connected to the cold mass, it can be concluded that the hot-spot temperature is acceptable when the stored energy is largely dissipated within the winding pack. Also, using a steel heater to initiate a quench to obtain a fast ramp-down in the case of an external anomaly or a quench in the current leads is possible, as the rampdown time, hot-spot temperature of various components and dynamic forces are at acceptable levels. 


\subsection{Conclusion}

THE chapter dealt with the thermal and electrical design of the conduction-

1 cooled NbTi MDS demonstrator magnet. The simulations involving the thermal budget of the system show that the magnet can be operated using a single cryocooler. The ability of the system to run conduction-cooled is beneficial for the performance as it avoids the need of a double-walled cryostat. Cool-down is possible in less than 16 days.

Calculations show that the cold mass is expected to stay below $3.1 \mathrm{~K}$ during a $15 \mathrm{~h}$ ramp, well below the $4.5 \mathrm{~K}$ limit imposed by stability- and thermal margin requirements of the superconductor.

The magnet is protected by diodes placed on the cold mass. These limit the voltage across the coils' terminals. The maximum internal voltage is $2 \mathrm{kV}$. The stored magnetic energy $(0.74 \mathrm{MJ})$ during a quench is deposited largely in the winding pack itself, reaching a rather safe hot-spot temperature of $135 \mathrm{~K}$. In the case of an external anomaly or a quench in the current leads, a heater on the cold mass can ensure a fast ramp-down of the system in around 3 seconds. After a quench the cold mass takes 5 days to cool down to the operating temperature again. 


\section{6 | Manufacturing of NbTi MDS racetrack coils}

This chapter describes the winding of the three MDS NbTi/Cu-based racetrack coils. The optimization of the geometry of the winding packs was described in Section 3.1, together with the composition of the coils. The coils were manufactured at the University of Twente. During the winding of the second coil, an electrical short occurred. The expected effects of this short on the expected operating behaviour of the magnet are analysed. 


\subsection{Introduction}

TITIALLY this chapter would have focused on the assembly and testing of the 1 demonstrator. Due to delays in the project, the assembly of the demonstrator is still in progress at the time of writing this thesis. The crucial first step in the realization of this demonstrator is the manufacturing of the NbTi-based racetrack coils which is discussed in Section 6.2; the diagnosis on an electrical short in one of the coils in Section 6.3; the estimated effects of the short on the operational behaviour of the magnet in Section 6.4 and lastly the attempt to find the value of the resistance of the short in Section 6.5.

\subsection{Coil winding procedure}

7 HIS section describes the winding of the three NbTi racetracks for the MDS demonstrator at the University of Twente. It was decided early on in the project that the windings would be impregnated to prevent conductor motion during operation, a commonly used strategy in superconducting magnets [38, 39]. In this way the entire winding pack becomes a single structural element, consisting of the conductor and the epoxy. Two distinct approaches were considered for the NbTi winding packs:

- The first, vacuum impregnation, is to wind the entire winding pack, place the coil in a vacuum mould and then to rely on the pressure difference between a resin reservoir at ambient pressure and the low pressure coil environment to fill all voids between turns with epoxy [102].

- The second, wet winding, is to apply uncured epoxy on the turns during the winding process, and to let the epoxy harden after completing a certain number of layers, after which winding can continue again [103].

The conductor should make up as large a fraction of the winding pack as possible. This improves the performance, since the number of ampere-turns in a given cross-section can be increased. A second advantage is that less mechanical energy can be stored in the epoxy, which reduces the possible heating of the winding pack when a crack is formed in the epoxy due to stress.

The highest theoretical packing factor of a round conductor is achieved with orthocyclic winding [56], where adjacent layers are shifted by half the diameter of the conductor and the packing factor can reach $90.7 \%$.

However, imperfections in the winding process reduce this packing factor. As more layers are wound on top of each other, errors accumulate and it becomes harder to position the conductor correctly. During winding trials with copper wire, a practical limit on the number of layers that could be wound with acceptable 
precision was found to be about $6^{1}$. Since a coil requires $69-70$ layers of 3234 turns each, this led to the choice of wet-winding, as this allows the winding process to be broken down into multiple sessions. Each session then could start with an error-free surface, provided that clamping during curing and subsequent cleaning are done correctly.

Stycast 2850FT Blue was selected as the epoxy resin, since compared to other epoxies its thermal expansion coefficient is relatively close to that of the conductor [42]. Catalyst $23 \mathrm{LV}$ was used, since it cures at room temperature and has a longer curing time than Catalyst 24 LV [104] and thus more layers could be completed in a single session. A packing factor of $75 \%$ was achieved. Protective breathing gear was worn during handling of uncured Stycast to prevent breathing in toxic fumes [104].

The winding set-up consist of three main parts, as shown in Figure 6.1:

- The feed-spool containing the conductor. This spool is placed on rollers and has a $60 \mathrm{~W}$ electromotor connected to its axis that acts as a brake;

- A wheel to apply tension on the wire. The conductor makes a full round along the perimeter of the wheel. The wooden wheel is covered with rubber for improved grip. A $115 \mathrm{~W}$ electro-motor provides the tension. Two additional (unpowered) wheels help to guide the wire;

- The main winding bench. This bench consists of a steel frame, a counterweight, a rotating section and a $150 \mathrm{~W}$ electromotor.

The conductor, with diameter $1.44 \mathrm{~mm}$, is wound on a stainless steel (SS304) mandrel, as shown in Figure 6.2. The mandrel remains part of the coils during their integration into the cryostat. It has a Teflon coating to prevent the Stycast resin from glueing the conductor to the mandrel.

As discussed in Section 3.2.2, the semi-circular coil heads are split into two sections to reduce the magnetic peak field on the conductor. Between the two sections of the heads, copper crescent-shaped pieces are inserted. In each coil, one of the two crescents incorporates a temperature sensor. Copper is chosen for its high thermal conductivity and because its thermal contraction is close to that of the winding pack (as discussed in Chapter 5).

Since a rectangular coil is easier to position and shim inside the aluminium cassette, copper end-pieces are put also on the ends of the winding pack to obtain a rectangular shape. On one of the flat faces of the winding pack, a $2 \mathrm{~mm}$ thick G11 plate includes a slot that carries the current lead. The current lead consists

\footnotetext{
${ }^{1}$ As more experience was gained during the winding of the coils, up to 10 layers could be wound in a single session.
} 


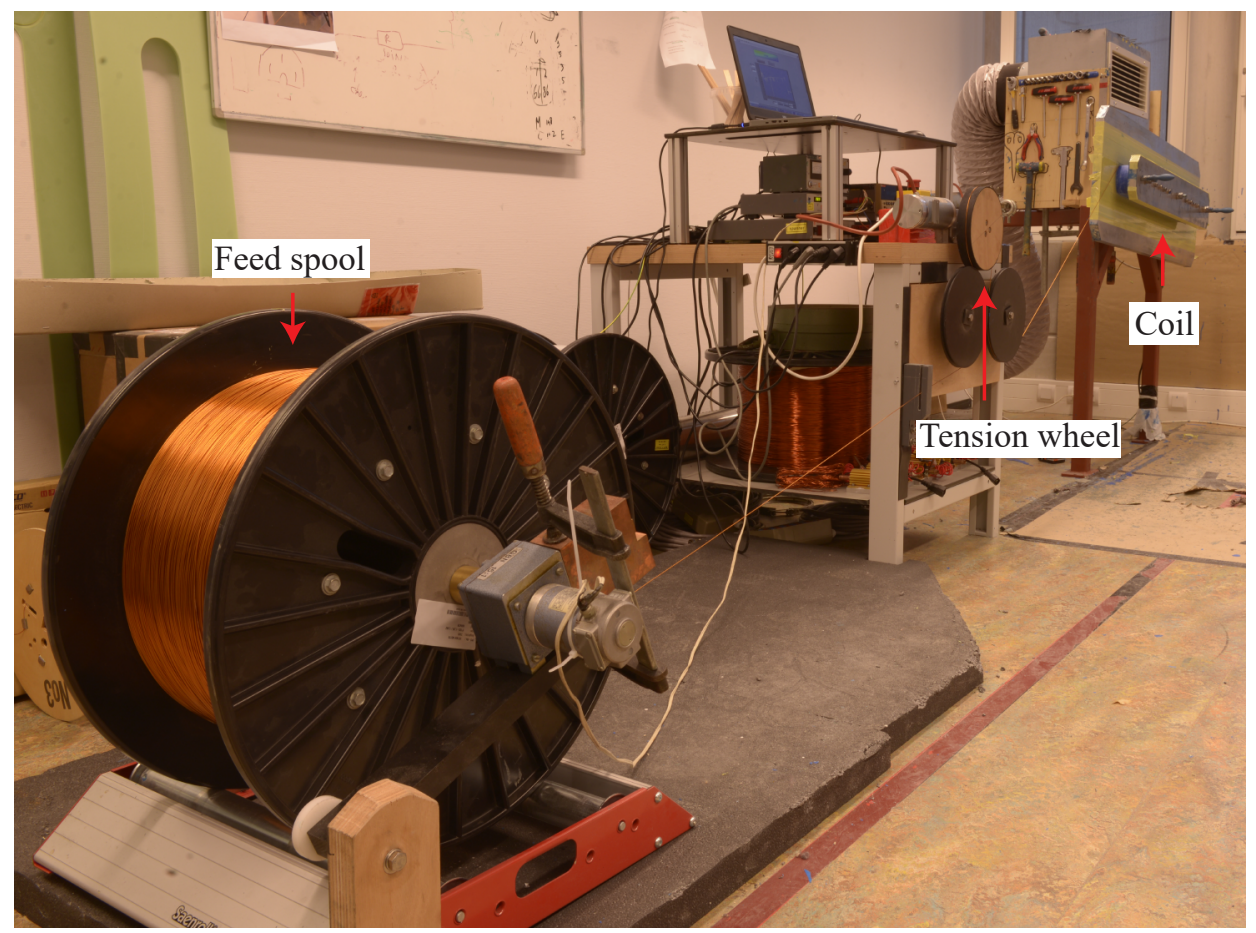

Figure 6.1: Winding set-up.

of two superconducting wires soldered together over their entire length, as shown in Figure 6.4b. On the other flat face, a $0.5 \mathrm{~mm}$ thick G11 plate is glued after winding.

The coil manufacturing consists of a cycle with three main phases:

(a) The actual winding phase, see Figure 6.3a-b. In this phase new layers are added to the winding pack. These are laterally confined by a main side plate and a smaller plate on the other side, both of aluminium. The smaller plate allows better control over the positioning of the windings thanks to improved visibility. During the winding process of each coil, this plate is twice replaced by a larger one when the winding pack becomes gradually thicker. Once a layer is completed, it is covered with Stycast 2850FT with a brush;

(b) The curing phase, see also Figure 6.3c-d. Once a winding session is finished, two straight aluminium beams are placed on the outer sections of the winding pack in order to compress the still-wet resin and to flatten these outer faces. The pushing force is applied by tightening a set of T-shaped 

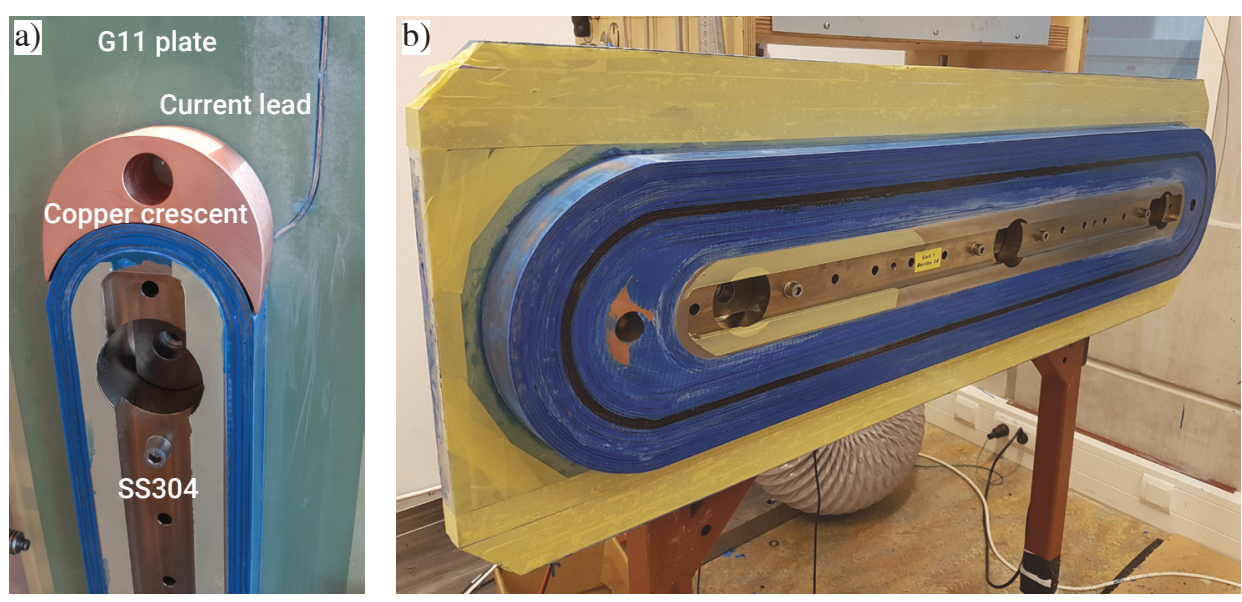

Figure 6.2: a) Copper crescent before further integration in the coil. The copper is surrounded by a layer of glass tape, smeared with Stycast. b) Coil 1 after winding roughly $75 \%$ of the turns. The black "belt" is due to the use of black Stycast during one winding session.

screws with trapezoid threads. The pressure applied by these beams is estimated to be $5 \mathrm{MPa}$. In this configuration the epoxy is allowed to cure.

To speed up the curing, 12 heaters rated at $600 \mathrm{~W}$ each are foreseen. Six of these are fixed to the main side-plate and six more can be placed on the removable side-plate in pairs of two. These heaters are powered with a temperature controller. The resistors are connected in two parallel strings of six each. The controller can supply up to $240 \mathrm{~V}$. The controller was foreseen to heat the main side plate to $70{ }^{\circ} \mathrm{C}$ for one hour, monitored with a Pt100 sensor inserted in a hole in this plate. The controller additionally restricts the supplied current by reading a second Pt100 that is connected to one of the heaters.

The resistors have connections that allow for water-cooling to enable a quick cool-down back to room temperature after curing;

(c) A cleaning phase, see also Figure 6.4. Once the epoxy is cured, the pushing beams and the smallest side plate are removed (Figure 6.4a) and excess Stycast is carefully removed with sanding paper and a scalpel (Figure 6.4b). The heads of the winding pack are rounded with a mixture of Stycast and glass beads (Figure 6.4c).

Initially it was anticipated that the whole cycle could be repeated once a day. However, this was found to be overly optimistic. Instead, the coils were wound on 
a one day winding, one day cleaning cycle. Curing took place overnight without use of heaters (Stycast 2850FT with Catalyst $23 \mathrm{LV}$ cures in half a day at room temperature[104]).

\subsection{Diagnosis of electrical short}

THE second coil that was manufactured turned out to contain an internal elec1 trical short. This section describes how this short was discovered, discusses possible causes and describes measures taken to avoid further occurrence of such shorts.

Before starting the winding of the second coil, the electrical resistance of the two remaining spools of NbTi wire was measured. According to the manufacturer, both spools contain a wire of a length of $7335 \mathrm{~m}$. Both resistance values were measured to be $150.4 \pm 0.1 \Omega$, corresponding to $\sim 20 \mathrm{~m} \Omega$ per meter conductor..

During the winding of the second coil, it was found that the electrical resistivity of the combination of the feeding spool and the coil had dropped $\sim 2 \%$, from 150.4 to $147.0 \Omega$. This measurement took place after 19 layers had been completed.

At first it was thought that this change could be explained by a variation of temperature in the winding room. The electrical resistivity of copper increases with $0.404 \% / \mathrm{K}$ around room temperature [105], i.e. the temperature would have been $\sim 6 \mathrm{~K}$ higher during the first spool measurement. It was decided to monitor this resistance on a more regular basis. In Figure 6.5 this measured resistance is shown as a function of the number of completed layers.

Excluding the initial spool value of $150.4 \Omega$, the mean resistance during the winding process was found to be $146.8 \Omega$, as indicated with a solid horizontal line in Figure 6.5. This figure also shows that if temperature fluctuations alone are responsible for the observed variation in resistance, a variation of $2 \mathrm{~K}$ around the average temperature needs to be assumed. This seems reasonable, since the winding room's windows were opened during the winding sessions for increased ventilation and coil 2 was wound in the winter. Also, the conductor may be below room temperature in the morning, due to its thermal inertia and the fact that the university buildings maintain a lower temperature outside standard working hours.

The lowest measured temperature of the winding mass was $17^{\circ} \mathrm{C}$, measured by inserting a Pt 100 thermometer in the main winding side-plate. The temperature of this plate was only measured sporadically and with only two significant digits.

However, to explain the difference between the initial $150.4 \Omega$ and the mean resistance value of $146.8 \Omega$, the conductor on the spool would have been $6 \mathrm{~K}$ above the standard conditions in the winding room. This is thought to be unlikely. 
Winding

a)

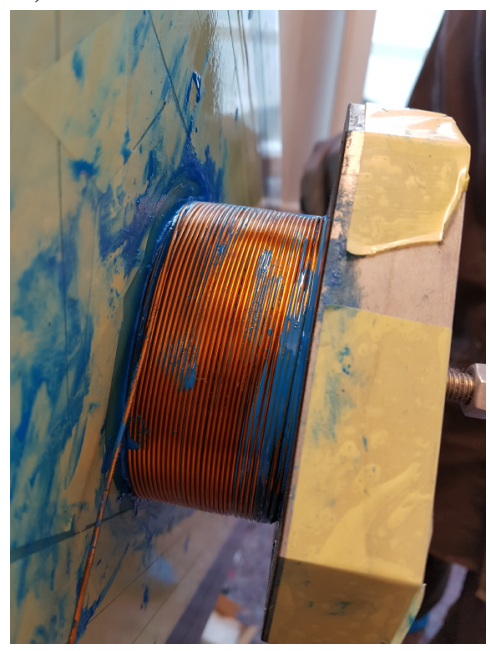

c)

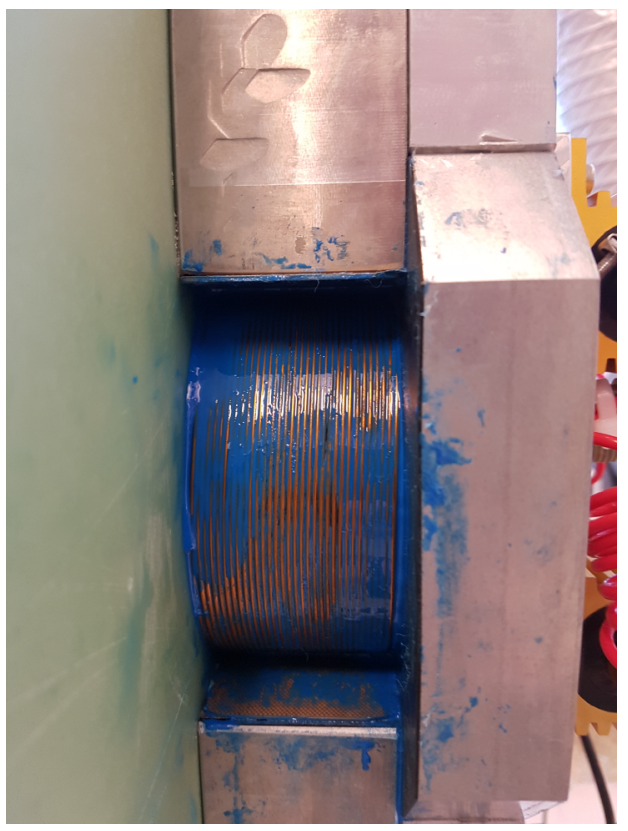

b)

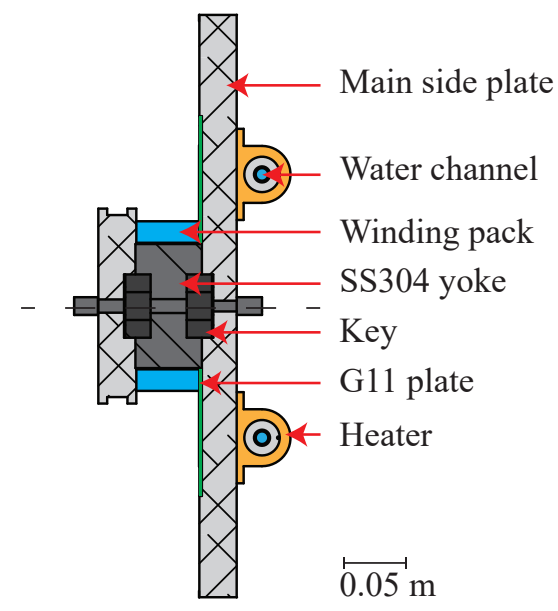

Curing

d)

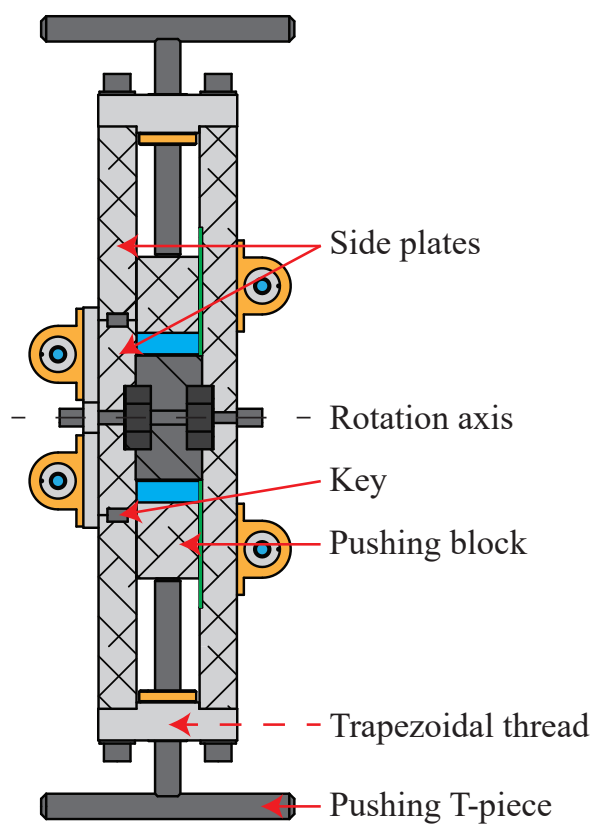

Figure 6.3: a) Winding phase. b) cross-section of tools in winding configuration. c) Curing phase. $d$ ) cross-section of tools in curing configuration. 


\section{Cleaning}

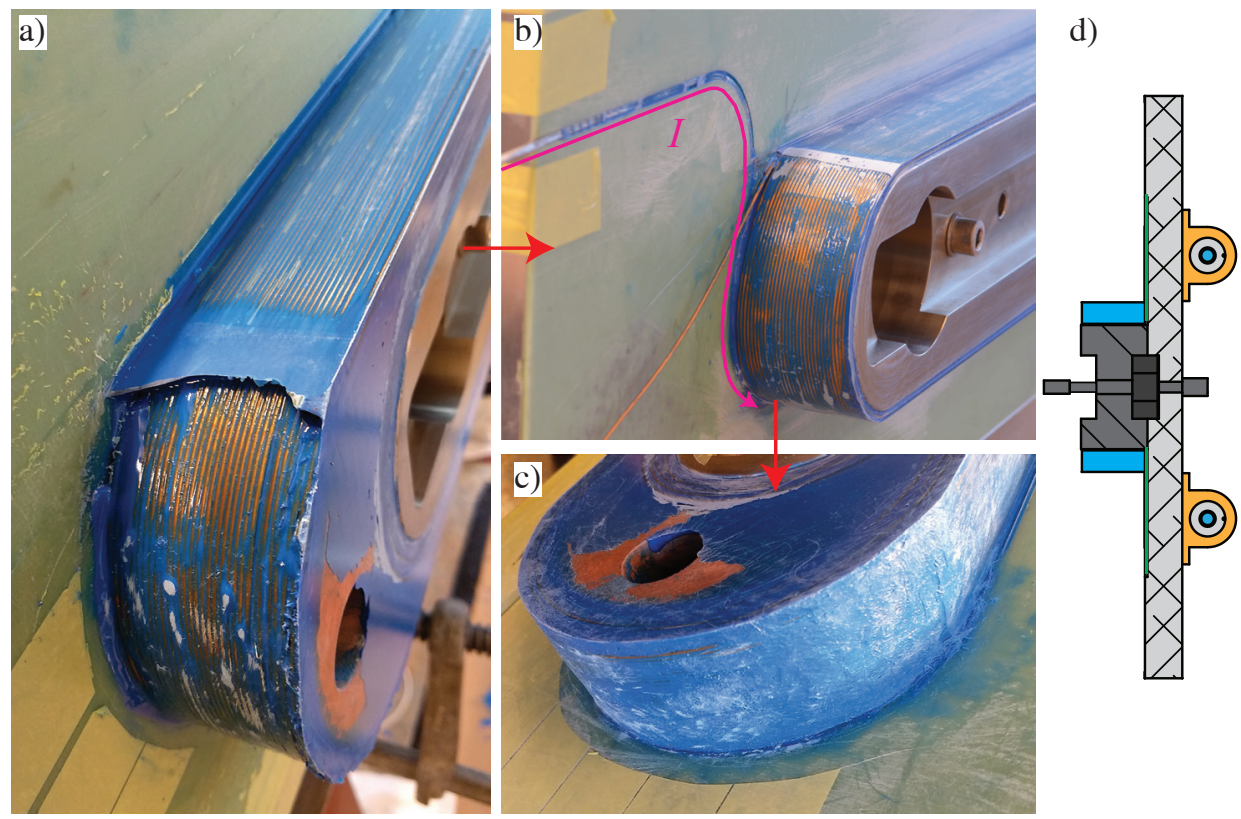

Figure 6.4: Winding pack during the cleaning phase, a) after removing the pushing beams, b) removal of excess Stycast, $\boldsymbol{c}$ ) after applying glass-loaded epoxy on the heads. d) cross-section of the winding tools in the cleaning configuration.

Thus it seems, based on these resistance observations, that an electrical short is present in coil 2 .

This short should be present somewhere between the first and the nineteenth layer. The copper crescent spacers, located between the 11th and 12th layer, were measured to be correctly electrically isolated from the winding pack, and thus do not seem to play a role in the short.

To estimate the number of turns involved in the short, we can assume that the resistance of the short itself is much smaller than that of the wire length in the shorted section. In that case the resistance decrease of the winding pack is equal to the resistance of the shorted section. This yields a value for the shorted section of 3.6 $\pm 1.2 \Omega$. The first layer has a turn length of $2.31 \mathrm{~m}$, the 19 th layer of $2.62 \mathrm{~m}$. This yields an estimate of the number of turns that is bypassed by the short ranging from 47 to 105, with a median of 74 turns. Based on this, a short between two successive winding layers seems likely, since this would involve between 2 and 68 turns. 


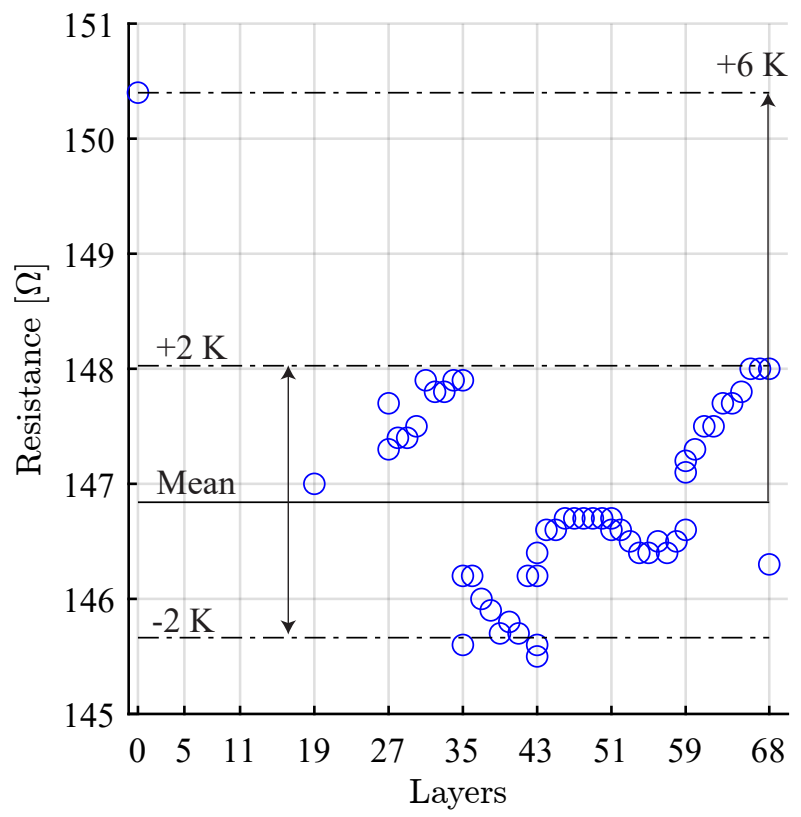

Figure 6.5: Measured electrical resistance of coil 2 in series with take-off spool 2, as a function of the already wound number of layers on coil 2. Vertical grid lines are placed at the start points of each winding session.

One possible cause of the short is the presence of a foreign hard particle in the winding pack. This could for example be a piece of solder, since the fume cupboard in which the two-component epoxy resin is mixed is also used for soldering. A piece of solder might thus have ended up in the Stycast.

Another possible foreign particle is a piece of hardened Stycast. During removal of excess cured Stycast from the winding pack and winding tools in between winding sessions (cleaning phase in Figure 6.4), a piece of hard Stycast might have ended up for example in the box of brushes used to apply wet Stycast. Such slivers of hardened Stycast can be sharp.

A second plausible type of cause is a double insulation failure, the accidental removal of the insulation layer in two turns in adjacent layers. This may happen for example at the location where the outgoing conductor and the winding pack separate, see Figure 6.6. This outgoing wire will always make an angle with the adjacent turns, with some epoxy filling the resulting gap. Careful removal of this epoxy 'arm-pit' after curing is required to prevent a later bump in the winding pack. It is not impossible that the insulation got damaged during this removal.

After discovering the short, care was taken (1) to prevent further inclusion 


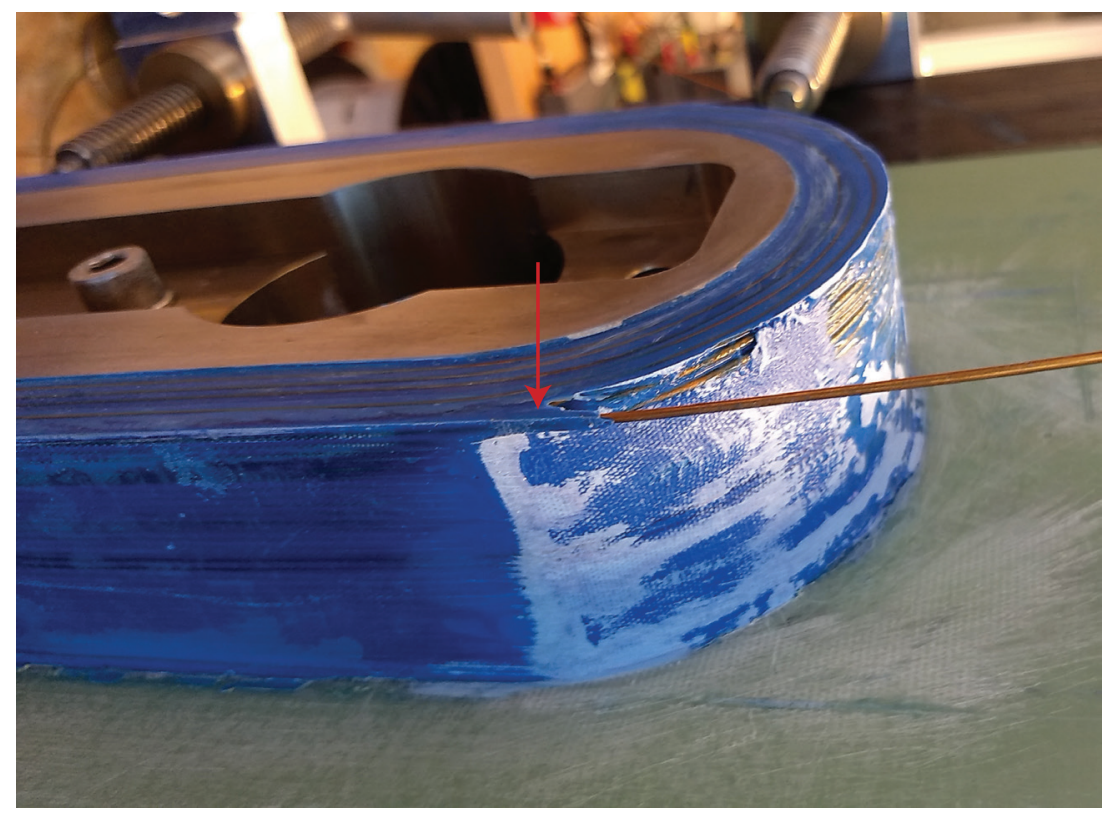

Figure 6.6: Close-up of winding pack during cleaning process. The arrow indicates the Stycast between the outgoing conductor and the main body. Removal of this Stycast might have lead to damage on the insulation layer of the conductor.

of hard particles in the winding pack, (2) to prevent damage of the insulation of the conductor during cleaning, and (3) to increase the frequency of resistance measurements.

Immediately after discovering the short, it was not clear if the second coil could still be used and some modelling effort was spent to answer this important question. The next section concerns itself with the possible consequences of an electrical short in one of the coils on the operating behaviour of the magnet. In this model we assume that the short consists of a copper-copper contact, but that there is no direct contact between NbTi filaments.

\subsection{Effects of electrical short}

SINCE the electrical short is not expected to be superconducting, steady-state $S$ operation of the magnet will not be affected as DC currents will follow the superconducting path. However, two potential effects during the ramping of a current in the coils have to be taken into account. The first is that in the shorted section $I_{\mathrm{c}}$ may be exceeded. The second is that the ohmic dissipation caused by 
this induced current in the short itself is too high.

To get an estimate of the possible magnitude of these unwanted effects due to the electrical short in coil 2, a simplified electrical model of the magnet is considered, as shown in Figure 6.7c.
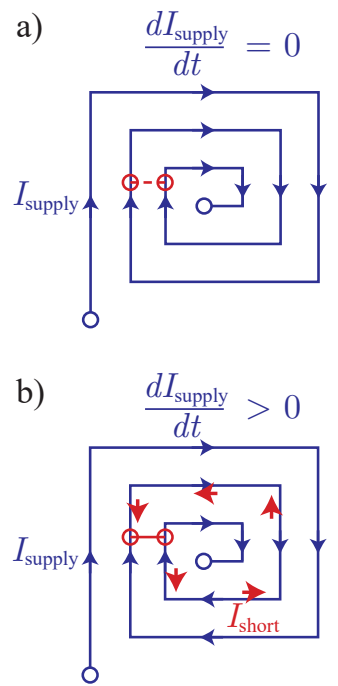

c)

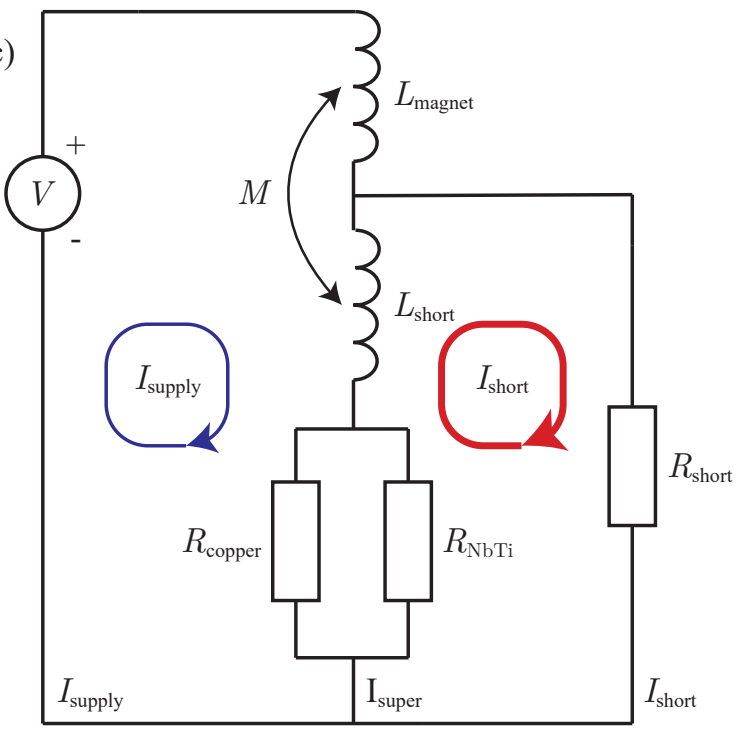

Figure 6.7: Schematic representation of the current path a) under steady state operation and $\boldsymbol{b}$ ) during a ramp of the excitation current $I_{\text {supply. }}$. In the later case, the induced layer-to-layer voltage drives an extra current component $I_{\text {short }}$ through the short. c) Simplified electrical representation of the magnet system with an electrical short.

To excite the magnet, a voltage source provides a potential difference across the coil terminals ${ }^{2}$. The magnet primarily consists of the three coils, lumped together to form the inductance $L_{\text {magnet }}$. Also part of the model circuit is the shorted section of coil 2, taking into account the inductance of the shorted section $L_{\text {short }}$ and the resistance of this shorted section. This resistance is modelled as two resistors in parallel, one representing the copper matrix $\left(R_{\text {copper }}\right)$ and the other the NbTi filaments $\left(R_{\mathrm{NbTi}}\right)$.

\footnotetext{
${ }^{2}$ The power supply also needs to supply a voltage across the current leads, and there are diodes present parallel to the coils, as explained in Section 5.3. As long as the voltage across the coils and diodes is sufficiently low, i.e. below the forward voltage of the diode string, the diodes can be neglected from this analysis.
} 
The shorted section is connected in parallel with a resistance $R_{\text {short }}$ that represents the bypass created by the electrical short.

The shorted section and the main coils are inductively coupled, which may be represented by a mutual inductance $M$. During the ramping of the magnet, this drives an unwanted current $I_{\text {short }}$ through the shorted section, indicated by the red loops in Figure 6.7c. The main coils and the shorted section thus act as a transformer. Since the number of turns in the intact coil section is much larger than in the shorted section, the ratio of the current in the shorted section to that in the intact coils can be large. The magnitude of $I_{\text {short }}$ can be controlled by the ramping rate of the magnet $d I_{\text {supply }} / d t$ (and thus by the voltage supplied by the power supply). An analysis is required to see what would be an acceptable ramping time for an MDS magnet with an electrical short.

The inductances of the MDS magnet with a short are calculated with a 3D FEM model. In the simulation it is assumed that the short is present in one of the two side coils. The shorted section is taken to comprise 68 turns, located in the inner two layers of the affected coil. The sum of the inductances and mutual inductances of the intact section is calculated to be $16.4 \mathrm{H}$. The shorted section has an inductance $L_{\text {short }} \approx 2.5 \mathrm{mH}$, and the mutual inductance $M$ with the intact sections is $62 \mathrm{mH}$. The corresponding coupling coefficient $k$ is 0.308 . This value is reasonable, since the shorted section and the bulk of the affected coil are closely coupled, whereas the shorted section and the other two coils or not. Thus a value slightly smaller than $1 / 3$ is to be expected.

The lumped electrical network with components as indicated in Figure $6.7 \mathrm{c}$ is solved to estimate the magnitudes of the currents in the main circuit, through the short, and through the shorted section, all as a function of time. The resistance of the shorted section can be estimated with reasonable accuracy, given the fact that its length is roughly known. The resistance of the filaments is modelled using a power law, with an $n$-value of 15 and a critical current of $2.5 \mathrm{kA}$ with a $10^{-5} \mathrm{~V} / \mathrm{m}$ criterion. The magnetic field dependence of the critical current is not taken into account in these rough estimates. The resistance of the short itself, however, is unknown. Therefore its value is swept over a wide range.

The power supply provides a voltage that is controlled based on the current the power supply is providing. We assume that this voltage has a constant value $V_{0}$ as long as the current is below the desired value of $300 \mathrm{~A}$, and zero at higher currents. The results shown in Figure 6.8 are calculated with $V_{0}$ set to $60 \mathrm{mV}$. This coincides with a ramp from zero to full current in about 23 hours.

Figure 6.8a shows the current as a function of time (1) delivered by the power supply $I_{\text {supply }}$, (2) flowing through the electrical short $I_{\text {short }}$ and (3) through the superconducting section $I_{\text {super }}$. $I_{\text {super }}$ rises to a negative value at first and then remains more or less constant. Once the ramp is completed, $I_{\text {super }}$ slowly decays 

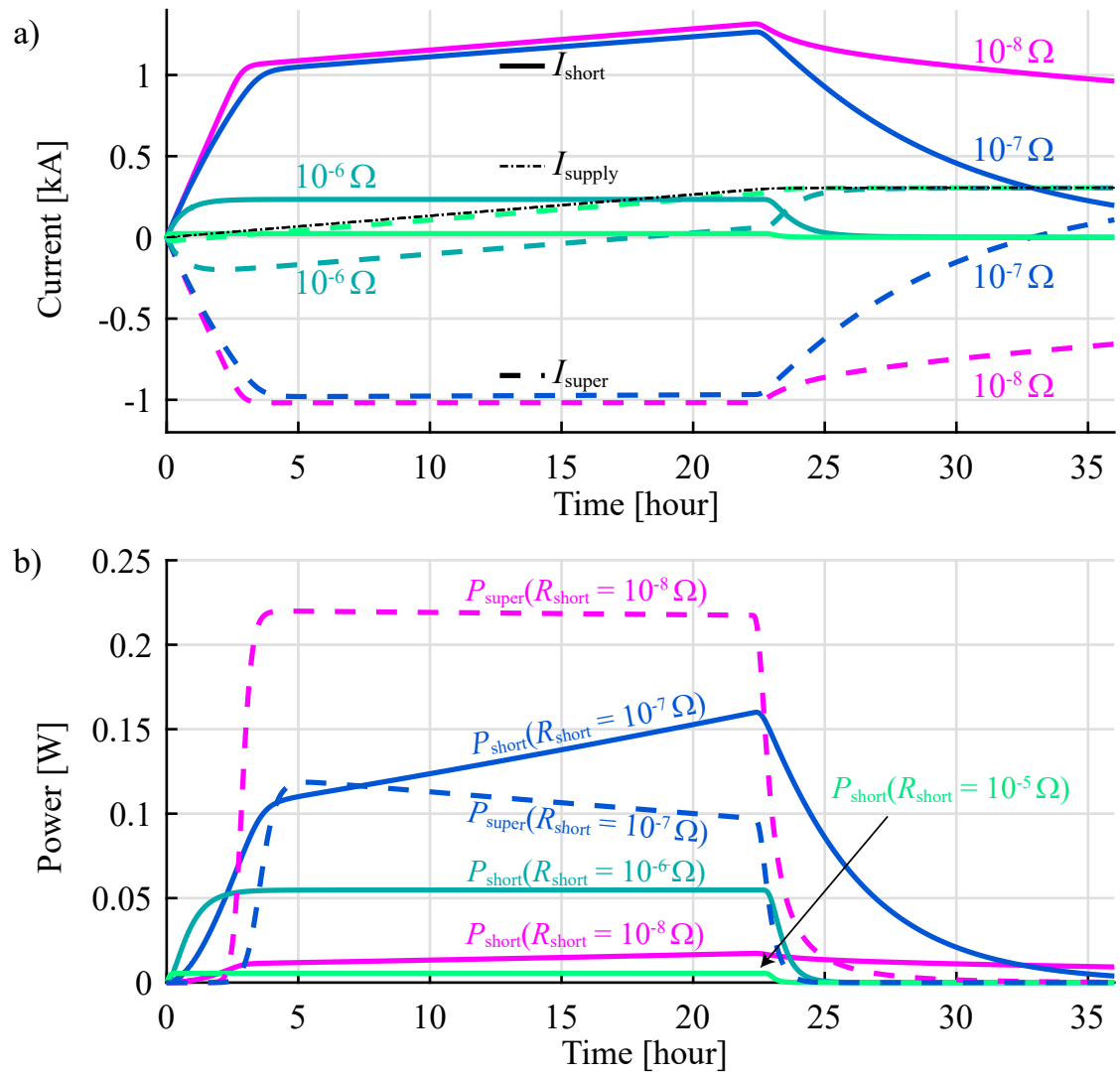

Figure 6.8: a) Currents versus time for a $60 \mathrm{mV}$ ramp, for various resistances of the electrical short $R_{\text {short. }}$. The dashed black lines indicate the current in the main circuit $I_{\text {supply. }}$. The solid coloured lines indicate the current through the electrical short $I_{\text {short }}$ and the dashed coloured lines through the shorted turns $I_{\text {super. }}$ b) Power dissipated as a function of time during a ramp with $60 \mathrm{mV}$, in the electrical short ( $P_{\text {short, }}$ solid lines) and in the shorted section itself $\left(P_{\text {super }}\right.$, dashed lines). Different coloured lines indicate different electrical short resistances $R_{\text {short }}$.

to the same value $I_{\text {supply }}$ as the main section. The current through the short $I_{\text {short }}$

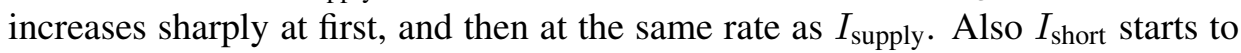
decay when the voltage supplied by the power supply starts to decrease. Once the ramp is completed, $I_{\text {short }}$ decays to zero.

The magnitude of these induced currents of course depends on the resistance $R_{\text {short }}$ of the short. If $R_{\text {short }}$ is smaller than $1 \mu \Omega,\left|I_{\text {super }}\right|$ can be significantly larger than $I_{\text {supply }}$. If $R_{\text {short }}$ is larger than around $10 \mu \Omega$ the induced current remains 
small relative to the supply current.

The power dissipated in the electrical short $P_{\text {short }}$ can be as high as $0.16 \mathrm{~W}$, as shown in Figure 6.8b. The highest dissipation in the short occurs for short resistances around $0.1 \mu \Omega$. The dissipation in the shorted section itself $P_{\text {super }}$ is also significant for short resistances of $0.1 \mu \Omega$ or lower, as the current in this section becomes significant. The dissipation goes up to $0.22 \mathrm{~W}$ for a $10 \mathrm{n} \Omega$ short.

There are two main risks associated with this dissipation. The first is that an increased heat load on the cryocooler means that the cryocooler temperature, and thus also the cold mass temperature will rise. However, as explained in Chapter 5 , some margin is available.

A second risk is that around the location of the short the winding pack can heat to a significantly higher temperature. Using the thermal model of the cold mass from Chapter 5, a $1.3 \mathrm{~K}$ local temperature rise in the coil is estimated for a $0.2 \mathrm{~mm} 50 \mathrm{~mW}$ "point" load. This corresponds to $R_{\text {short }}$ value of $1 \mu \Omega$. The temperature profile is mainly affected near the short itself. A benefit could be that the increase in temperature is located in such a small volume that no minimum propagation zone is created.

The big unknown in the analysis in the value of $R_{\text {short }}$ at cryogenic temperatures. Assuming a contact length between the two damaged turns of at most $10 \mathrm{~mm}$ and a direct copper-copper contact, a lower bound of around $0.7 \mu \Omega$ can be placed [106]. This is a low enough resistance to cause problems. Thus room-temperature measurements of the affected coil were performed in an attempt to obtain a rough estimate of the short resistance. These measurements are described in the next section.

\subsection{Room-temperature impedance}

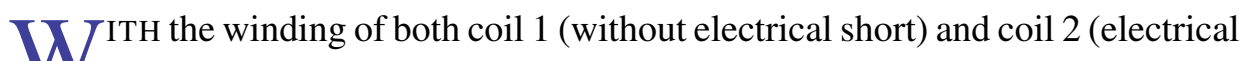
short suspected) completed, the impedance of both coils was measured. Figure 6.9 shows the impedance as a function of frequency.

Also shown is the overall impedance of a simple RLC-network in which the coil is represented by a resistor in series with an inductor, together with a capacitor placed parallel to the first two elements (Figure 6.10a) [107]. The capacitance $C_{\text {coil }}$ was set to $0.9 \mathrm{nF}$, to match the experimental data of coil 1 . Both the measured data and the model show a peak in the impedance around $2.5 \mathrm{kHz}$. The phase starts at $0^{\circ}$ (resistance dominated), rises to around $+90^{\circ}$ (inductive), and switches to $-90^{\circ}$ (capacitative) above $2.5 \mathrm{kHz}$.

The main differences in the data measured on the second coil are a shift in the impedance peak from 2.5 to $\sim 3 \mathrm{kHz}$, a broad dip in the phase between $30 \mathrm{~Hz}$ and 

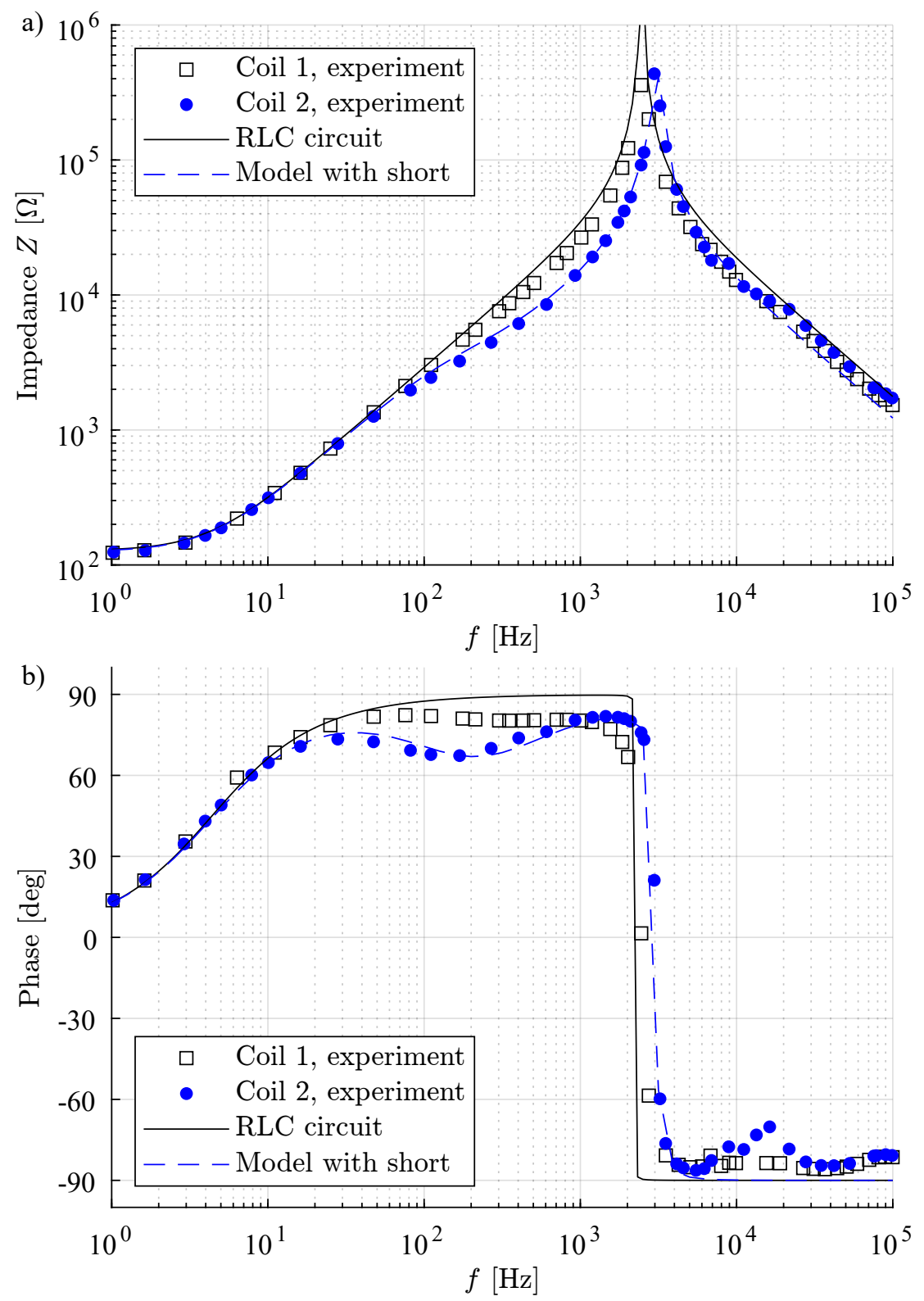

Figure 6.9: a) Frequency-dependent impedance of coils 1 and 2. b) Phase of the impedance of coils 1 and 2.

$1 \mathrm{kHz}$ that is absent in coil 1 , and a feature around $12 \mathrm{kHz}$ in coil 2 that is present in coil 1 but of smaller magnitude. 


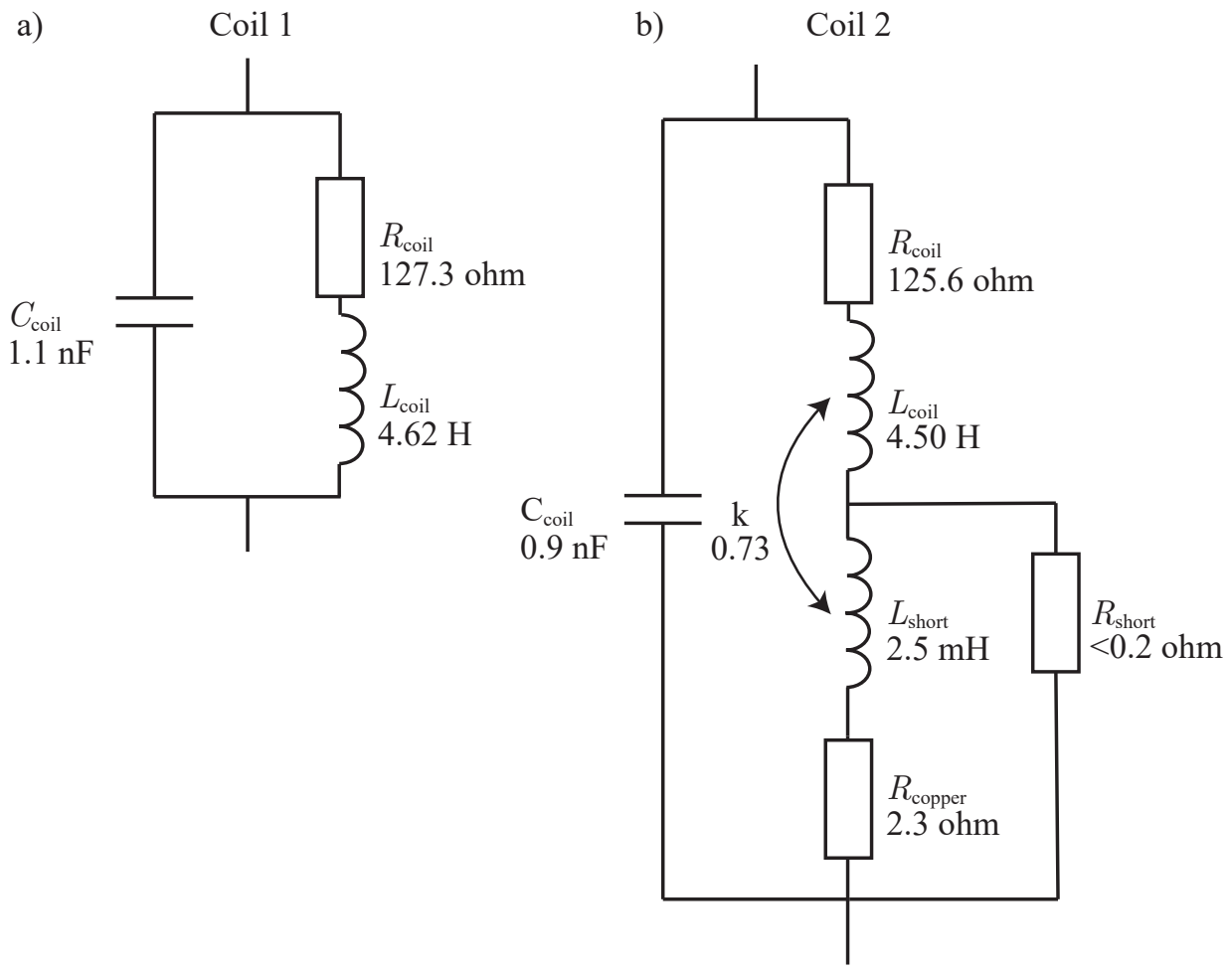

Figure 6.10: a) RLC model representing the intact coil, b) Electrical model representing the coil with a shorted section. The values correspond to best fits to the measured data presented in Figure 6.9.

The phase dip in the inductive regime can be reproduced by an electrical model of the shorted coil as shown in Figure 6.10b. However, the sensitivity of the fit to the capacitance, inductance, and mutual inductance is much larger than its sensitivity to the short resistance. Only an upper bound on $R_{\text {short }}$ of around $0.2 \Omega$ can be placed.

The copper in the matrix of the conductor has a RRR of around 110, as discussed Section 3.1.4. Assuming that $R_{\text {short }}$ has the same temperature dependence, this places an upper bound on the short resistance at the cold mass at $1.8 \mathrm{~m} \Omega$. This is not precise enough to determine a safe ramping speed of the magnet.

Subdividing the capacitance into a part in parallel with the main coil and an other part in parallel with the short does not significantly change the results.

The feature in the phase signal at $12 \mathrm{kHz}$ is not reproduced with the simple model. Possibly the tables on which the coils lay during the impedance measure- 
ment contained various amounts of metal.

It was concluded that no accurate estimate of the electrical short resistance could be obtained at room temperature. It was also decided that the damaged coil will still be used in the magnet, which can be ramped to a current of a few amperes after cool-down. Using a Hall-sensor, the relaxation of the magnetic field in time due to the influence of the short can be measured. By obtaining an estimate of the short resistance from this cryogenic test, a safe ramping speed will be extracted.

\subsection{Conclusion}

THIS chapter described the winding process of the NbTi MDS racetrack coils 1 at the University of Twente. The coils are wet-wound in stages. Stycast 2850FT epoxy resin is applied on the turns during the winding. After winding up to 10 layers, the straight sections of the racetrack are pressed and allowed to cure so that a flat surface is obtained upon which the next winding section can start.

During the winding of the second coil, an electric short was accidentally created, likely between two adjacent winding layers and possibly due to a foreign particle or to the post-cure cleaning operation. To estimate the possible impact on this coil's operation, the effects of such an electrical short of unknown resistance on the ramping behaviour of the magnet are analysed with the aid of an electrical network model. The result is that the magnet in all likelihood should not be ramped within one day, due to the heat generated by the large electrical currents in the shorted section and the short itself.

Room-temperature measurements were performed in an attempt to measure the magnitude of the resistance of the electrical short, but the sensitivity of the model used in the data analysis to the value of short resistance was too low for an accurate estimate. Instead, a relaxation test of the magnetic field upon excitation is proposed to establish a safe excitation protocol. 


\section{7 | Conclusion \& outlook}

$\mathrm{T}^{\mathrm{N}}$ this thesis the design of the first superconducting magnet system for magnetic density separation (MDS) is described. MDS is a relatively new separation technology that allows to separate a particle mixture of multiple non-magnetic components based on their mass density in one single process, using a magnetic field gradient and a ferrofluid. Such a technology is expected to contribute strongly to the renewable use of resources and to a cyclic economy.

Compared to the permanent magnets used in state-of-the-art MDS systems, the higher magnetic field strength and increased design space offered by superconducting electromagnets in principle offer multiple advantages: (1) higher magnetic forces and hence the possibility to separate heavier materials; (2) wider poles, enabling deeper fluid beds and thus enhanced separation resolution; and (3) more dilute ferrofluids, leading to a significant reduction in operational cost. The superconducting MDS system described in this thesis aims to provide a test bed to demonstrate these features.

There are two distinct strategies to move a particle feed stream through an MDS fluid bed. In the first one the ferrofluid is simply pumped round and drags the feed particles across the separation chamber, while in the second one a horizontal magnetic force component is created by tilting the coil array with respect to the horizontal plane. The present demonstrator system is of the second type and is designed to separate shredded electronic materials, illustrating the first advantage mentioned above.

The thesis focuses on the design of its superconducting magnet, including the cryostat and system assembly. At the time of writing this thesis, the realization of the demonstrator has been started and is expected to be completed by the middle of 2021. A first step in this process was the manufacturing of the superconducting coils, which is also described in this thesis.

In Chapter 2 the ideal magnetic field for MDS is derived. Such a magnetic field has a magnitude gradient that chances monotonically in one single direction. It was shown that only a magnetic field with a magnitude that decays exponentially with the distance to the magnet satisfies these requirements. Mathematically, this 
magnetic field is generated by a pure harmonic sheet current.

This ideal harmonic current distribution can in practise be approximated with an array of racetrack coils, albeit at the cost of introducing undesired magnitude variations in the magnetic field in a plane parallel to the magnet system. These parallel variations can be minimized by choosing the relative dimensions of the coils such that the width of the rectangular current-carrying sections exactly equals one third of the periodicity $\lambda$ of the current distribution. $\lambda$ also determines the exponential decay rate of the magnetic field, and for the demonstrator was chosen to be $0.6 \mathrm{~m}$. Also the effect of a finite winding pack thickness, as opposed to an idealized 2D sheet current, on the magnetic field quality is analysed and described in Chapter 2. Thicker winding packs have a more advantageous ratio of the useful magnetic field in the ferrofluid to the current-limiting peak-field on the superconductor, but this effect eventually saturates, rendering also overly thick winding packs economically inefficient.

In Chapter 3, an infinite array of racetrack coils as derived in Chapter 2 is taken as a starting point to determine the optimum number and type of racetrack coil. An distinction is made between "wide" and "narrow" coil families, and it is shown that narrow coils generate less undesired ripple in the perpendicular gradient of the magnetic field. Using the same optimization metric, also edge effects caused by truncation the infinite array to a finite number of coils are analysed. Three narrow coils are found to be optimal in a tilted system for a wide range of tilt angles. The demonstrator design therefore consists of three racetrack coils, each with a total width of $300 \mathrm{~mm}$ (i.e. $\lambda / 2$ ) and $1 \mathrm{~m}$-long straight legs. Each coil has around 2230 turns of $\mathrm{NbTi} / \mathrm{Cu}$ conductor, and will carry a current of $300 \mathrm{~A}$ at or just below the nominal operating temperature of $4.5 \mathrm{~K}$. The resulting magnetic peak field on the conductor is $5.2 \mathrm{~T}$ while the average magnetic field at the bottom of the ferrofluid bed, $50 \mathrm{~mm}$ from the coils' surface, is $2 \mathrm{~T}$. The average vertical field gradient at the bottom of the fluid is of magnitude $20 \mathrm{~T} / \mathrm{m}$.

In Section 3.2, the relation between the maximum obtainable vertical gradient in the fluid with the thickness of the winding pack is derived. This relation is in general non-monotonic, since a thicker winding pack implies higher Lorentz forces and hence requires a sturdier support structure, increasing the distance between coils and ferrofluid. For the $\mathrm{NbTi} / \mathrm{Cu}$ conductor used in the demonstrator the expected performance is near optimal $(21 \mathrm{~T} / \mathrm{m})$. It should also be noted that the chosen cooling strategy (conduction-cooling) increases this performance by roughly a factor two compared to bath-cooling, since it avoids the need for a double-walled cryostat.

Also state-of-the-art technical superconductors other than $\mathrm{NbTi}$ are considered in order to estimate the potential performance of future MDS magnets. A distinction is made between "conservative" designs, i.e. designs that use a similar 
mechanical enclosure as the NbTi demonstrator, and "rather optimistic" designs that assume a mechanical enclosure that does not substantially increases the coilto-fluid distance when the magnet becomes more powerful. $\mathrm{MgB}_{2}$ is found to perform similar to $\mathrm{NbTi}$ at $4.5 \mathrm{~K}$. $\mathrm{Nb}_{3} \mathrm{Sn}$ allows to generate a magnetic field gradient of $36 \mathrm{~T} / \mathrm{m}$ with the conservative design and more than $60 \mathrm{~T} / \mathrm{m}$ with the optimistic assumption. A ReBCO-based MDS system operating at $4.5 \mathrm{~K}$ will also deliver about $35 \mathrm{~T} / \mathrm{m}$ with the conservative design and over $75 \mathrm{~T} / \mathrm{m}$ with the optimistic one. Compared to the demonstrator presented in this work, such a nearly four-fold increase in performance clearly illustrates how there is a lot to be gained, especially by further optimization of the mechanical enclosure of the coil system. Performance estimates of ReBCO-based MDS systems operating at higher temperatures are also given.

With the optimal electromagnet configuration and magnetic field profile for the demonstrator determined, the magnetic attracting force between coils and ferrofluid can be derived. In Section 3.3 it is shown how this attracting force, of the order of $8 \mathrm{kN}$ in normal operation, overcomes the $500 \mathrm{~kg}$ weight of the cold mass, causing the loading state of its support system to change from compressive to tensile upon magnet excitation. In the case of ferrofluid instability, with all ferromagnetic nanoparticles segregating at the bottom of the fluid bed, this force may further rise to about $34 \mathrm{kN}$. Also other risks are considered, such as partial drainage of the ferrofluid, which can create a torque of up to $1.6 \mathrm{kNm}$ on the system.

In Chapter 4 these and other mechanical aspects of the MDS demonstrator are covered in more detail. The main mechanical design challenge specific to the MDS application is to keep the distance between the ferrofluid and the coils minimal, thus maximizing the magnetic field gradient in the fluid.

First, the mechanical lay-out of the cryostat is discussed. Its vacuum vessel consists of a D-shaped main chamber with attached to it an instrumentation turret that houses electrical feedthroughs and the actual cryocooler. Especially the flat top-plate of the vacuum chamber experiences sizeable inward forces due to the combined ambient pressure and the attraction of the ferrofluid to the magnet. Using internal support columns between this top plate and the curved bottom of the chamber, the plate can nevertheless be kept relatively thin $(20 \mathrm{~mm})$.

In the brief Section 4.3, the effective mechanical properties of the winding packs are estimated. These estimates serve as input for the simulations of the mechanical behaviour of the cold mass presented in Section 4.4. The cold mass comprises the coils but also their cassette, an aluminium enclosure that shrinkfits around the winding packs during cool-down. This thermal pre-compression offsets the outward directed Lorentz force generated during excitations and thus keeps the coils immobile. The required pre-compression level is the main factor 
that determines the thickness of the cassette material.

In Section 4.5 is described the design on the support structure that keeps the cold mass in place within the cryostat. This structure consists of four vertical pillars and four horizontal tie rods. These elements are optimized to provide adequate strength while minimizing the conductive heat in-leak, leading to the choice for fibre-reinforced G11 composite cylinders with appropriate dimensions. Not only static loading, including anomalous operation as discussed above, but also the dynamic behaviour of the system is analysed to ensure that the magnet survives external vibrations. Especially transport and installation are found to be critical in this respect.

In Chapter 5 the thermal and electrical design of the system is presented.

First, the dominant heat loads are identified and an estimated heat-budget is drawn up. The main conclusion is that the system can be operated at $4.5 \mathrm{~K}$ using just a single cryocooler. Next, a more refined thermal lumped-element model is solved to get a more detailed insight in the heat fluxes and temperatures in the system. This analysis leads to the introduction of high-purity aluminium thermal heat drains at the bottom of the cold mass keep to the thermal gradient across the coils below $70 \mathrm{mK}$. The model also predicts the dynamic thermal behaviour of the system, showing that the initial cool-down will take 16 days and that full recovery after a quench will require 5 days.

For quench protection, varistors are connected in parallel to the coils and physically bolted to the cold mass. When a quench is detected, a breaker opens the main electrical circuit and the current flows through the diodes and the coils. The $0.75 \mathrm{MJ}$ energy stored in the magnetic field is thus dissipated mainly within the quenching coil(s). This is feasible because of the heat capacity of the winding packs. The expected maximum temperature within the windings is $135 \mathrm{~K}$ and the maximum internal voltage $2 \mathrm{kV}$. The current decays in $3 \mathrm{~s}$.

Also in the case of a quench in the superconducting current leads or in the case of an external emergency, the switch is opened and the coils are quenched on purpose by a resistor that is installed in series with the diodes. The response time of this solution was found to be adequate.

In Chapter 6 the wet winding process of the NbTi racetrack coils is presented in some detail. Also the effects of an internal electrical short that was accidentally introduced in one of the coils are analysed with an electrical network model. Due to this short, the magnet cannot be ramped up to full field within less than one day. Higher ramp rates induce too high ohmic dissipation in the short itself and excessive currents in the shorted section of the winding pack.

The main recommendation for future superconducting MDS systems is the further exploration of other technical superconductors than $\mathrm{NbTi}$, specifically Re$\mathrm{BCO}$ and $\mathrm{MgB}_{2}$. A 'universal' optimal superconducting MDS system, suited for 
the separation of all types of feed streams, does not exist. Instead the optimal material choice depends on the specific application. A detailed cost analysis balancing capital cost, dominated by the magnet system, against operational cost, dominated by the replenishing of lost ferrofluid, is required for the selection of the specific superconducting material for a specific MDS application.

The larger cooling power available by operating at a higher temperature allows to simplify the cryogenic system and to increase the strength of the support structure significantly. In addition, thermal contact resistance plays a smaller role at higher temperatures and the thermal conductivity of copper is higher. Thus higher heat loads do not necessarily result in a higher temperature gradient.

As shown in Chapter 3, the gain in performance of ReBCO-based magnets compared to the presented $\mathrm{NbTi}$ demonstrator can be significant if a different type of mechanical enclosure can be devised in which the coil-to-fluid distance does not need to increase with increasing Lorentz force. 


\section{A | Scaling laws}

THIS appendix discusses the relationship between the electrical engineering 1 current $J_{\mathrm{e}}$ and magnetic flux density $B$ and temperature $T$ of several technical superconductors. These relations are referred to as scaling laws. They are of the form $J_{\mathrm{e}}(B, T)$.

\section{A.1 NbTi}

$7 \mathrm{O}$ estimate the engineering critical current density of $\mathrm{NbTi}$ as a function of 1 magnetic field and temperature a scaling law was used as suggested by Bottura [108]:

$$
J_{\mathrm{e}}(B, T)=J_{\text {ref }} \frac{C_{0}}{B} b^{\alpha}(1-b)^{\beta}\left(1-t^{m}\right)^{\gamma},
$$

where

$$
\begin{aligned}
t & =\frac{T}{T_{\mathrm{c} 0}}, & b=\frac{B}{B_{\mathrm{c} 2}}, \\
B_{\mathrm{c} 2} & =B_{\mathrm{c} 20}\left(1-t^{m}\right) . &
\end{aligned}
$$

$B_{\mathrm{c} 20}$ and $T_{\mathrm{c} 0}$ can be attributed to a physical interpretation as the upper critical magnetic field and upper critical temperature. Bottura suggests a value of 1.7 for $m$.

The coefficients, presented in Table A.1, for the scaling law used in this thesis are matched to the NbTi wire from Bruker used as the conductor for the MDS demonstrator.

The critical current of the wire as a function of applied magnetic field was measured at $5 \mathrm{~T}$ by Bruker and at 6 to $10 \mathrm{~T}$ at the University of Twente (UT), see Table A.2. No data was obtained at $5 \mathrm{~T}$ at the UT due to limitations of the current leads of the used insert. The wire has a copper-to-non-copper ratio of 1.35.

The University of Twente measurement data are corrected for self-field 
Table A.1: Coefficient values used in the scaling law of NbTi (Eq. A.1).

\begin{tabular}{lllllllll} 
Symbol & $C_{0}$ & $\alpha$ & $\beta$ & $\gamma$ & $m$ & $B_{\mathrm{c} 20}$ & $T_{\mathrm{c} 0}$ & $J_{\text {ref }}$ \\
\hline Unit & {$[\mathrm{T}]$} & {$[-]$} & {$[-]$} & {$[-]$} & {$[-]$} & {$[\mathrm{T}]$} & {$[\mathrm{K}]$} & {$\left[\mathrm{kA} / \mathrm{mm}^{2}\right]$} \\
Value & 40.6 & 0.812 & 1.23 & 2.3 & 1.7 & 13.9 & 9.3 & 3.78
\end{tabular}

Table A.2: Critical current of the NbTi wire used in the demonstrator, measured at $4.2 \mathrm{~K}$. The $5 \mathrm{~T}$ data-points were measured by the manufacturer Bruker, the other data-points were taken at the University of Twente (UT), using a $10^{-5} \mathrm{~V} / \mathrm{m}$ electric field criterion on a $1.2 \mathrm{~m}$ long sample wound on a barrel.

\begin{tabular}{lrrr} 
Lab & Background field [T] & Critical current [A] & $n$-value [-] \\
\hline Bruker & 5.0 & 1921 & 59 \\
UT & 6.0 & 1573 & 23 \\
UT & 7.0 & 1141 & 19 \\
UT & 8.0 & 716 & 24 \\
UT & 9.0 & 373 & 18 \\
UT & 10.0 & 64 & 9
\end{tabular}

(0.443 mT/A) and used to fit Eq. A.1. The Bruker data-point is not used as the self-field is not known. As the UT measurements are all performed in a liquid helium bath at atmospheric pressure, $\gamma$ can not be determined from the data and instead a value of 2.3 is taken from [108]. Using a least-mean-square fit, the coefficients in Table A.1 are obtained.

Figure A.1 shows the engineering critical current density as a function of magnetic field. The fit to the data-points at $4.2 \mathrm{~K}$ is shown, as well as the scaling law at $6.5 \mathrm{~K}$. This $6.5 \mathrm{~K}$ line indicates the maximum allowed current density in order to achieve a $2 \mathrm{~K}$ temperature margin for the MDS magnet at an operating temperature of $4.5 \mathrm{~K}$. 


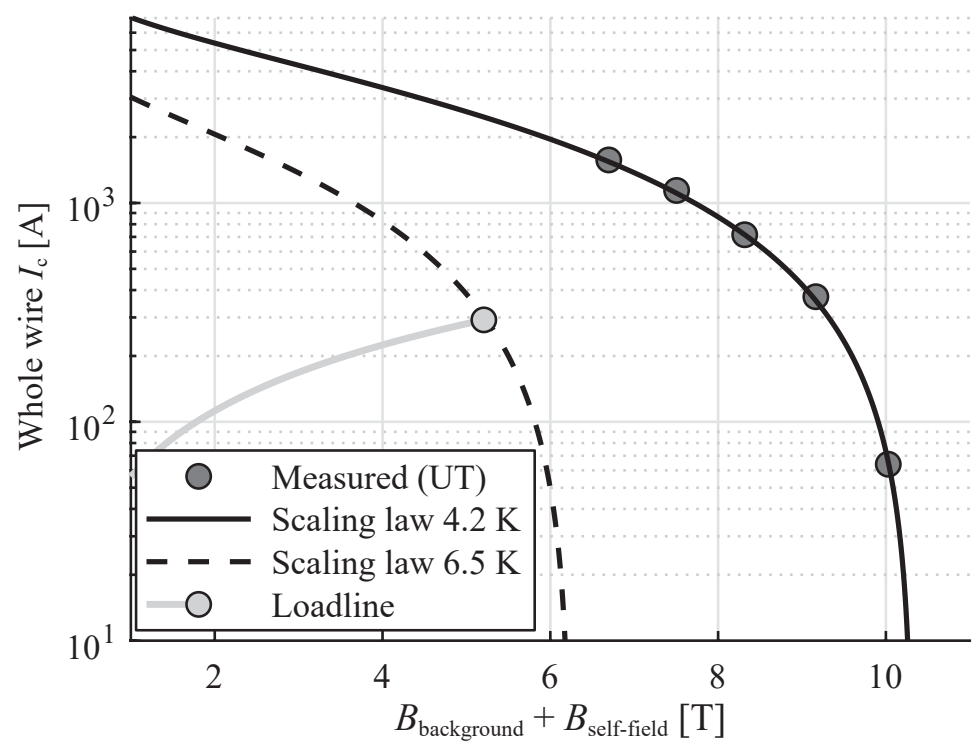

Figure A.1: Critical current of NbTi wire used in the MDS demonstrator, as a function of magnetic field. Dark markers show measurements, to which the solid black line is fitted. This fit allows (via Eq. A.1) a predication of the performance at $6.5 \mathrm{~K}$ (dashed line).

\section{A.2 $\mathrm{Nb}_{3} \mathrm{Sn}$}

$T^{\text {HE }}$ used scaling law for $\mathrm{Nb}_{3} \mathrm{Sn}$ has the form [109]

$$
J_{\mathrm{e}}(B, T)=J_{\text {ref }} \frac{C_{0}}{B} b^{\alpha}(1-b)^{\beta}\left(1-t^{m}\right)\left(1-t^{2}\right),
$$

with $t, b$ and $B_{\mathrm{c} 2}$ as defined by Eq. A.2 and Eq. A.3. The $\mathrm{Nb}_{3} \mathrm{Sn}$ scaling law coefficients are based on a fit to a characteristic dataset measured at the University of Twente on a strand produced by Oxford Instruments, Superconducting Technology (OST) [109]. The $C_{0}$ coefficient is scaled to match the minimum required conductor performance of LHC MQXF at $12 \mathrm{~T}$ and $4.22 \mathrm{~K}$ : a whole-wire critical current density of at least $1.1 \mathrm{kA} / \mathrm{mm}^{2}$ [110]. 
Table A.3: Coefficient values used in the scaling law of $N_{3} S n$ (Eq. A.4).

\begin{tabular}{llllllll} 
Symbol & $C_{0}$ & $\alpha$ & $\beta$ & $m$ & $B_{\mathrm{c} 20}$ & $T_{\mathrm{c} 0}$ & $J_{\text {ref }}$ \\
\hline Unit & {$[\mathrm{T}]$} & {$[-]$} & {$[-]$} & {$[-]$} & {$[\mathrm{T}]$} & {$[\mathrm{K}]$} & {$\left[\mathrm{kA} / \mathrm{mm}^{2}\right]$} \\
Value & 66 & 0.5 & 2 & 1.52 & 33.77 & 16.16 & 1.114
\end{tabular}

\section{A.3 $\mathrm{MgB}_{2}$}

$\mathrm{F}^{\mathrm{OR} \mathrm{MgB}_{2} \text { an empirical scaling law of the form [111] }}$

$$
J_{\mathrm{c}}(B, T)=J_{\mathrm{ref}} C_{0}(1-t) \exp \left(-\frac{B}{B_{\mathrm{p}}}\right),
$$

is used, where

$$
B_{\mathrm{p}}=B_{\mathrm{p} 0}(1-t),
$$

and where $t$ as defined by Eq. A.2 and $B_{\mathrm{p} 0}$ a fitting constant.

The $\mathrm{MgB}_{2}$ scaling law coefficients, presented in Table A.4, are fitted to datapoints measured on a Hypertech wire in 2015 at the University of Twente, see Figure A.2. The sample had a length of only a couple of centimetres and currents were moderate $(<500 \mathrm{~A})$, so the self-field was estimated to be negligible compared to the background magnetic field. The whole-wire critical current density is $183 \mathrm{~A} / \mathrm{mm}^{2}$ at $5 \mathrm{~T}$ and $4.2 \mathrm{~K}$.

The least-mean-square fit shows deviations from the measurements at higher temperatures, but this is not thought to be a problem as the evaluation of the potential $\mathrm{MgB}_{2}$ performance in an MDS system is performed for a $4.5 \mathrm{~K}$ operating temperature.

Table A.4: Coefficient values used in the scaling law of $M g B_{2}$ (Eq. A.5).

\begin{tabular}{lllll} 
Symbol & $C_{0}$ & $B_{\mathrm{p} 0}$ & $T_{\mathrm{c} 0}$ & $J_{\text {ref }}$ \\
\hline Unit & {$[\mathrm{T}]$} & {$[\mathrm{T}]$} & {$[\mathrm{K}]$} & {$\left[\mathrm{kA} / \mathrm{mm}^{2}\right]$} \\
Value & 8.644 & 2.68 & 38.6 & 0.183
\end{tabular}




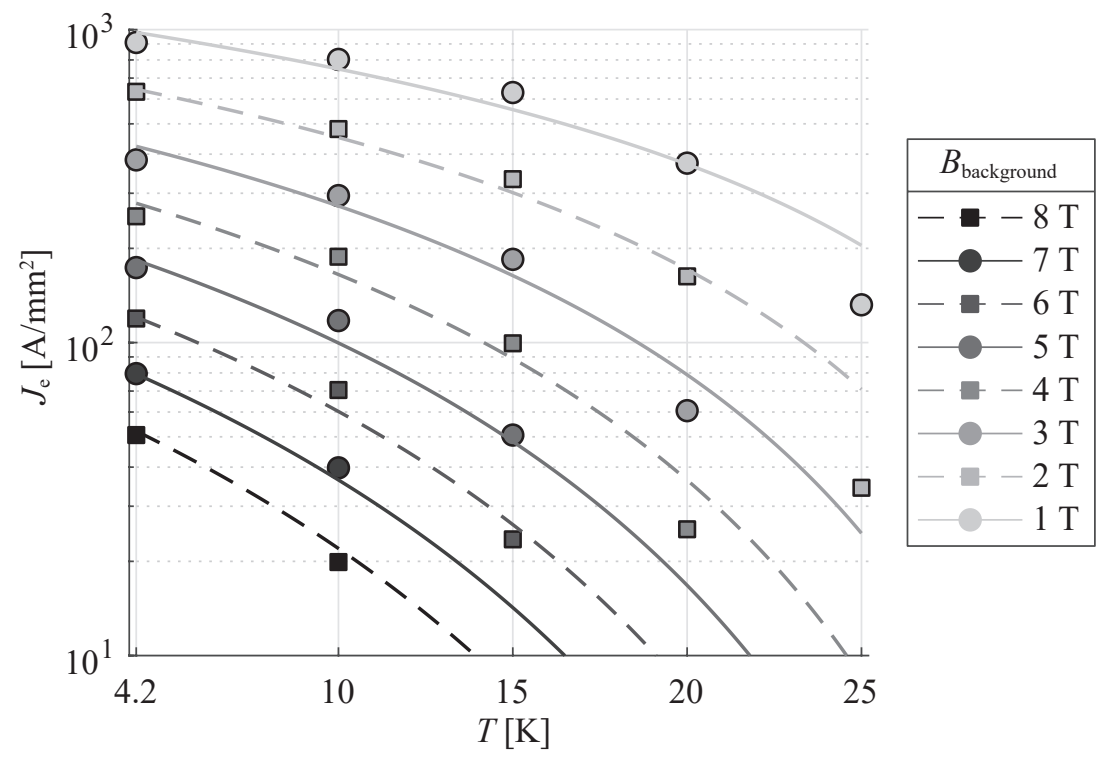

Figure A.2: $M g B_{2}$ whole wire critical current density as a function of magnetic field and temperature. Markers indicate data-points and lines indicate a fit of Eq. A.5 to these points.

\section{A.4 ReBCO}

THE scaling law for ReBCO describes the critical current density as a function 1 of temperature and a perpendicular magnetic field [46]. This magnetic field orientation yields the lowest performance and is thus a worst-case:

$$
J_{\mathrm{e}}\left(B_{\perp}, T\right)=J_{\mathrm{ref}} \frac{C_{0}}{B} b^{\alpha}(1-b)^{\beta}\left(1-t^{m}\right)^{\gamma},
$$

where

$$
b=\frac{B_{\perp}}{B_{\mathrm{i}, \perp}}, \quad \quad B_{\mathrm{i}, \perp}=B_{\mathrm{i} 0, \perp}\left(1-t^{m}\right),
$$

and with $t$ as defined by Eq. A.2. $B_{\mathrm{i} 0, \perp}$ can be interpreted as the upper irreversibility field for a perpendicular orientation.

The coefficients used in this thesis are given in Table A.5. These were obtained [46] by fitting the scaling law to measurements on a Fujikura tape. $C_{0}$ was increased by $50 \%$ to represent a tape with a $100 \mu \mathrm{m}$ substrate instead of the original $150 \mu \mathrm{m}$ tape. This brings the engineering critical current density to 
$0.68 \mathrm{kA} / \mathrm{mm}^{2}$ at $12 \mathrm{~T}(\perp)$ and $4.2 \mathrm{~K}$.

Table A.5: Coefficient values used in the scaling law for ReBCO (Eq. A.7).

\begin{tabular}{lllllllll} 
Symbol & $C_{0}$ & $\alpha$ & $\beta$ & $\gamma$ & $m$ & $B_{\mathrm{i} 0, \perp}$ & $T_{\mathrm{c} 0}$ & $J_{\mathrm{ref}}$ \\
\hline Unit & {$[\mathrm{T}]$} & {$[-]$} & {$[-]$} & {$[-]$} & {$[-]$} & {$[\mathrm{T}]$} & {$[\mathrm{K}]$} & {$\left[\mathrm{kA} / \mathrm{mm}^{2}\right]$} \\
Value & 54.6 & 0.5 & 2.5 & 2.44 & 1 & 140 & 93 & 0.68
\end{tabular}




\section{B I Magnetic field profile}

7 HIS section contains figures that detail the magnetic field profile $H\left(x^{\prime}, y, z^{\prime}\right)$ 1 of the superconducting MDS demonstrator at the nominal operating current of $300 \mathrm{~A}$. The coordinate system is slightly translated from that used in the rest of the thesis, as the $z^{\prime}$-coordinate is shifted so that $z^{\prime}=0$ corresponds to the bottom of the fluid bed. 

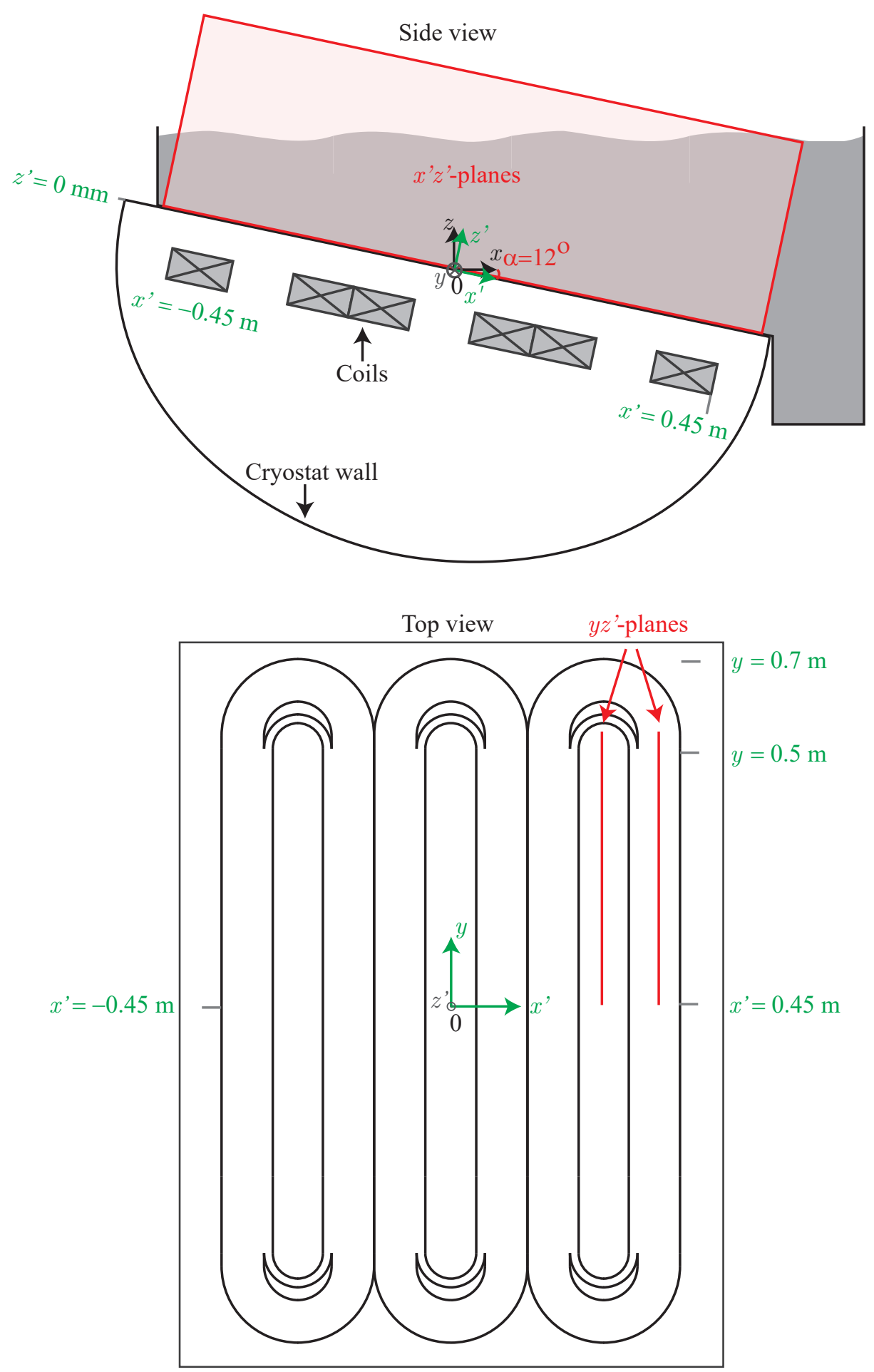
Stray field [mT]

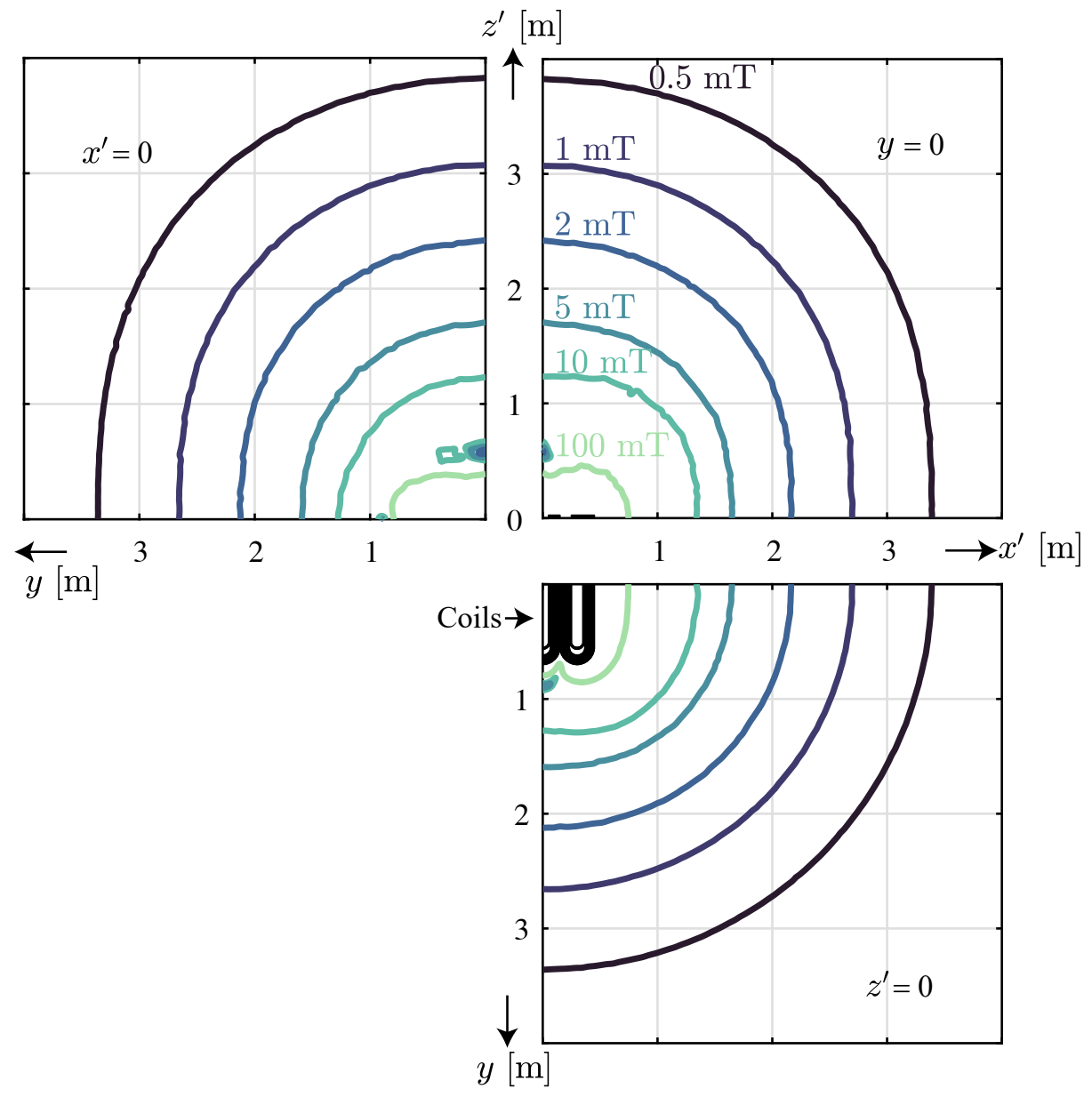




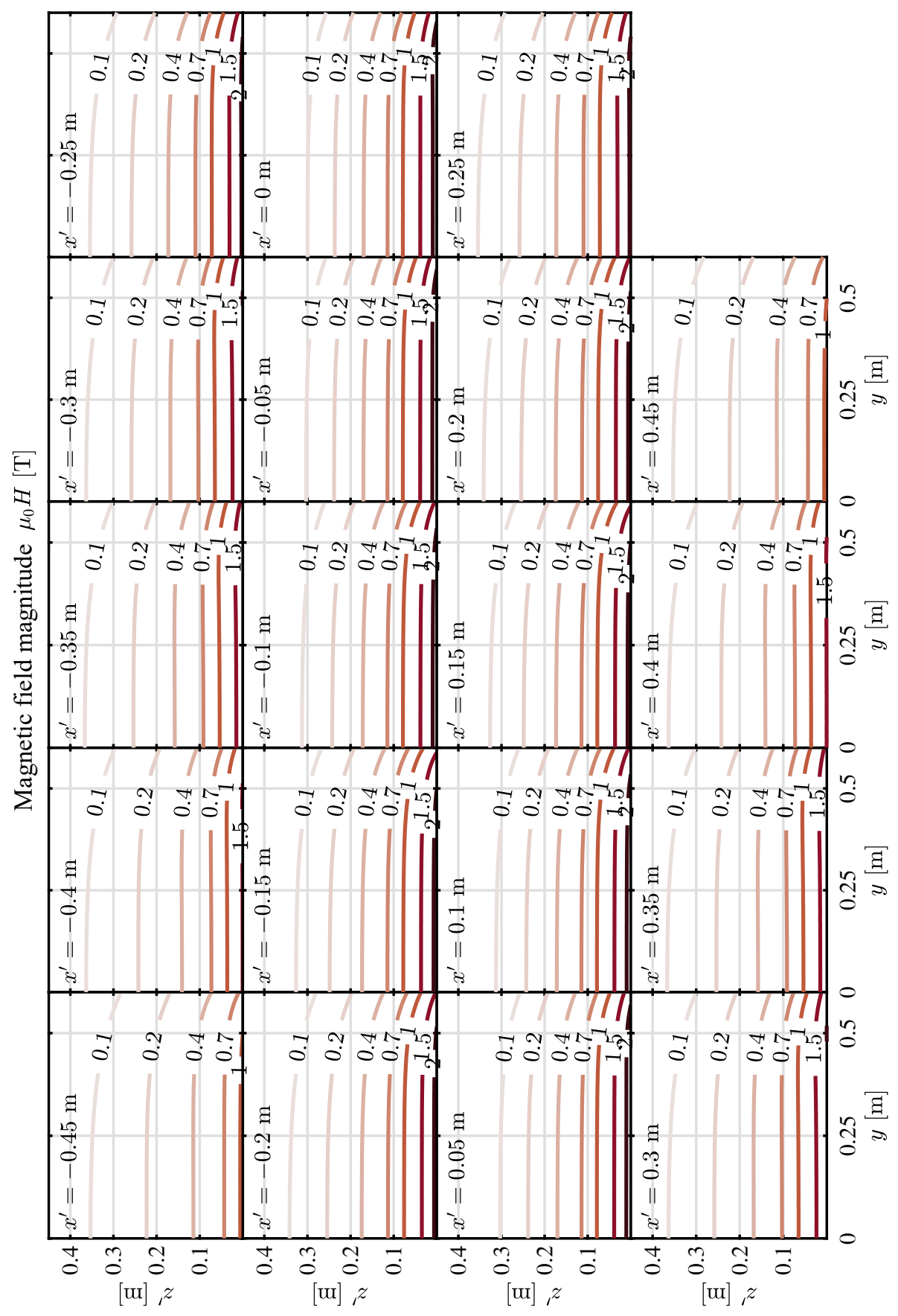




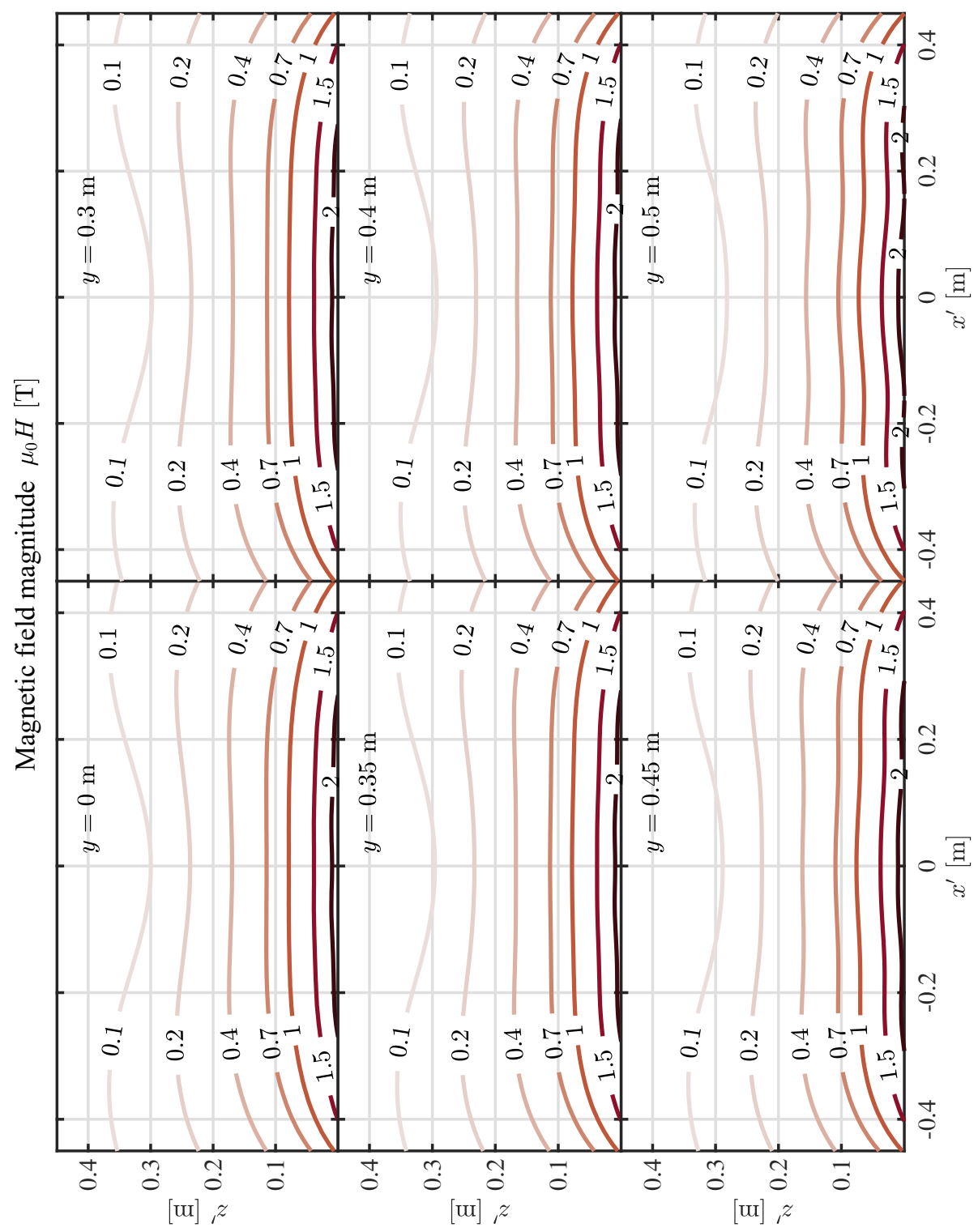




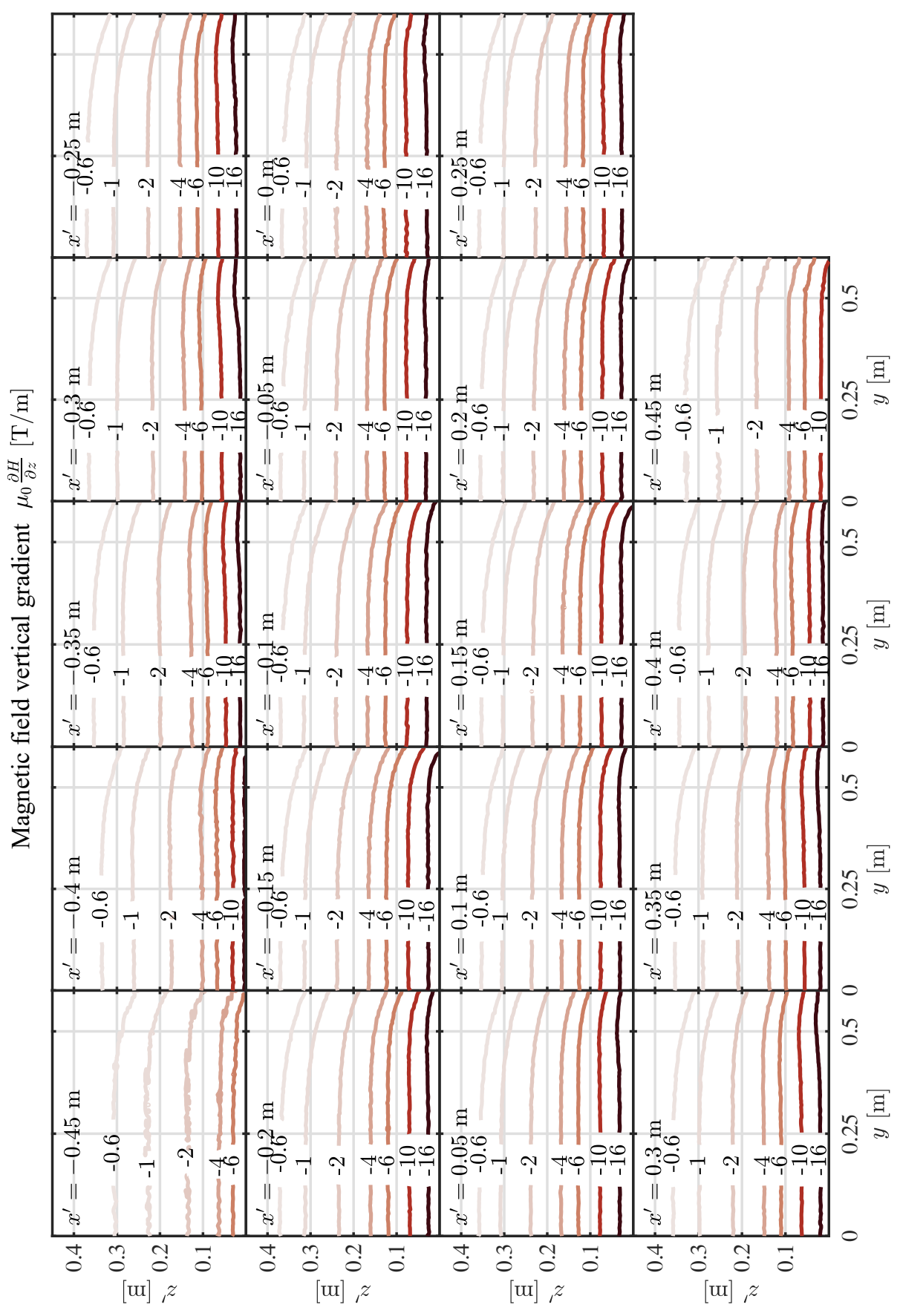




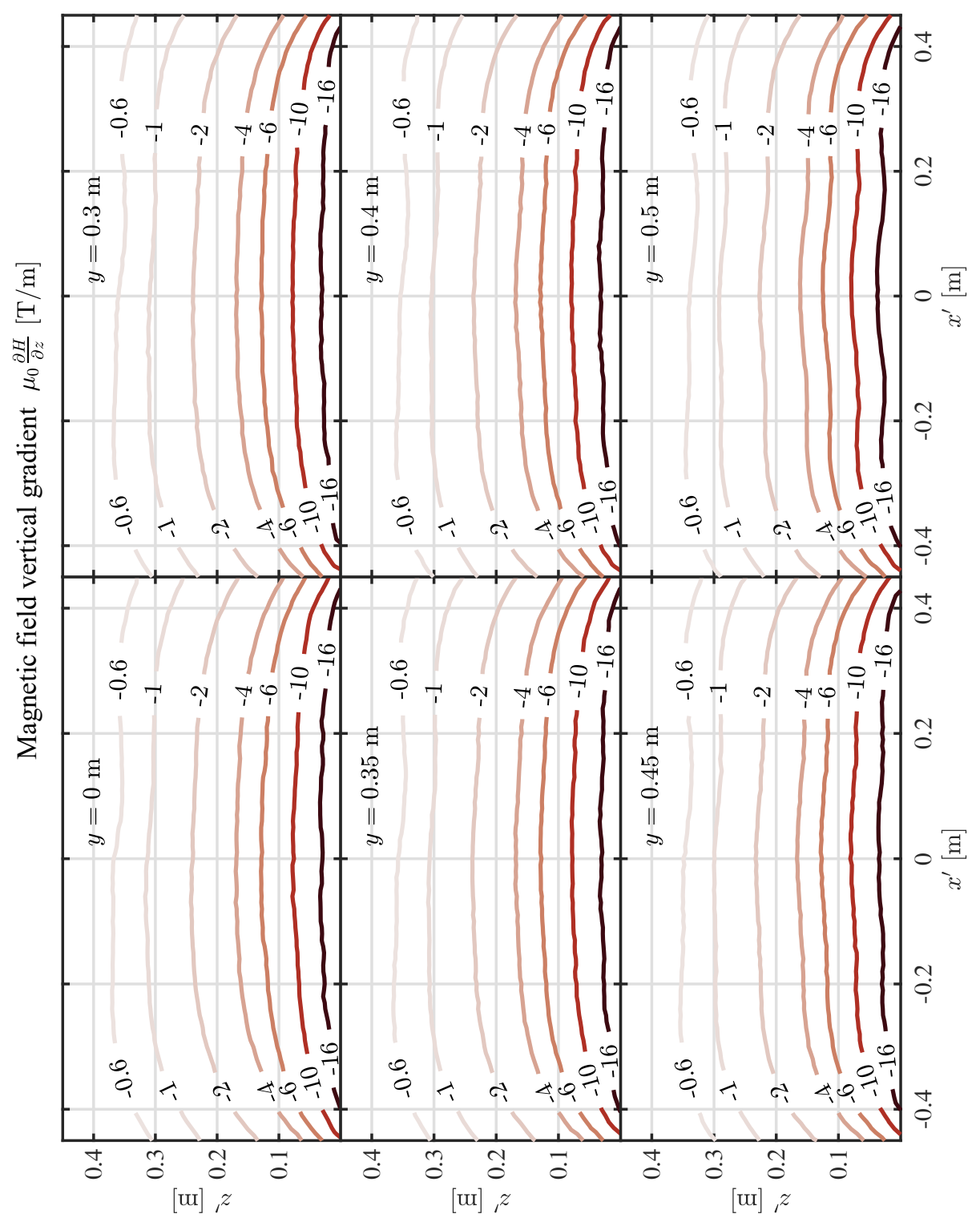




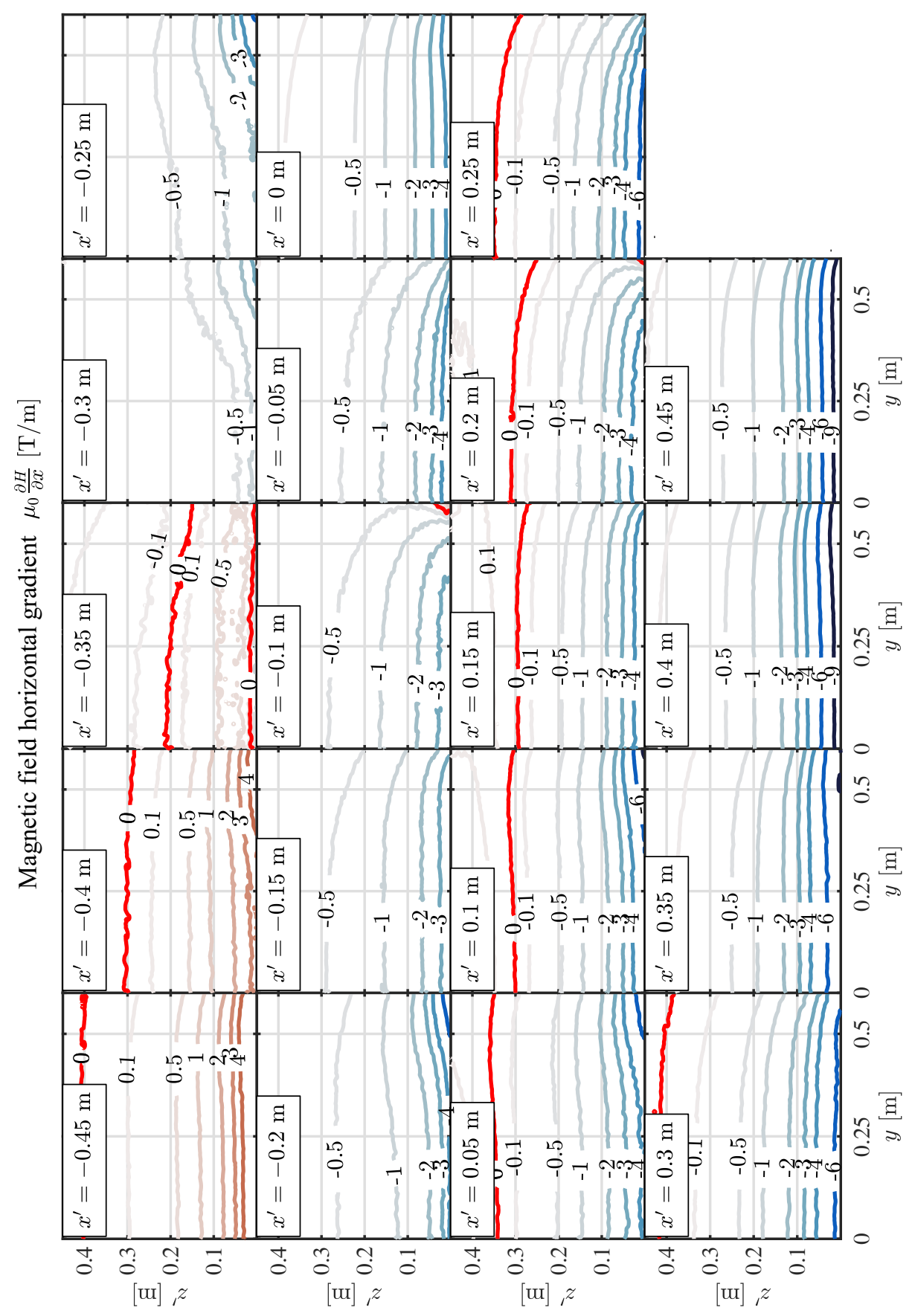




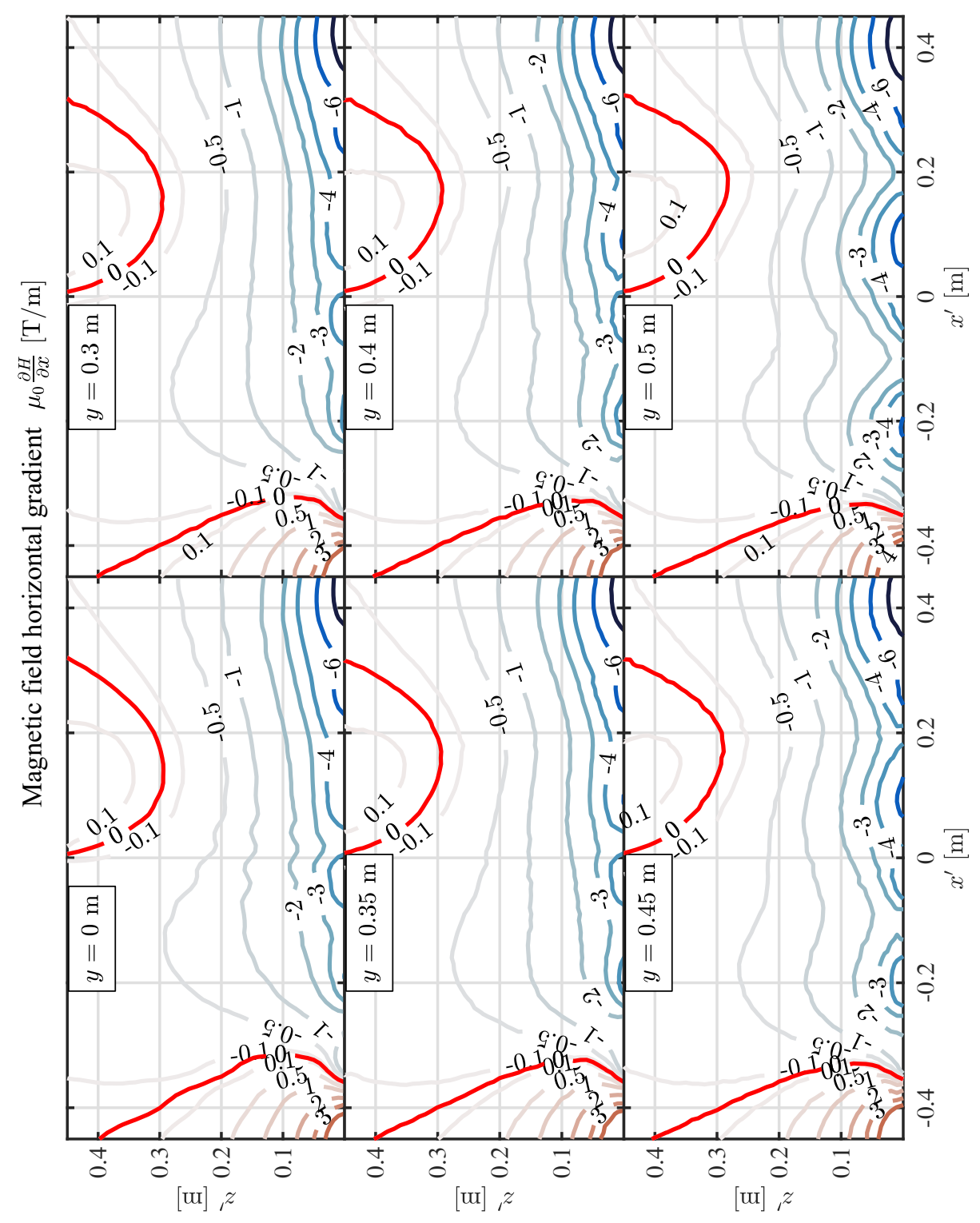




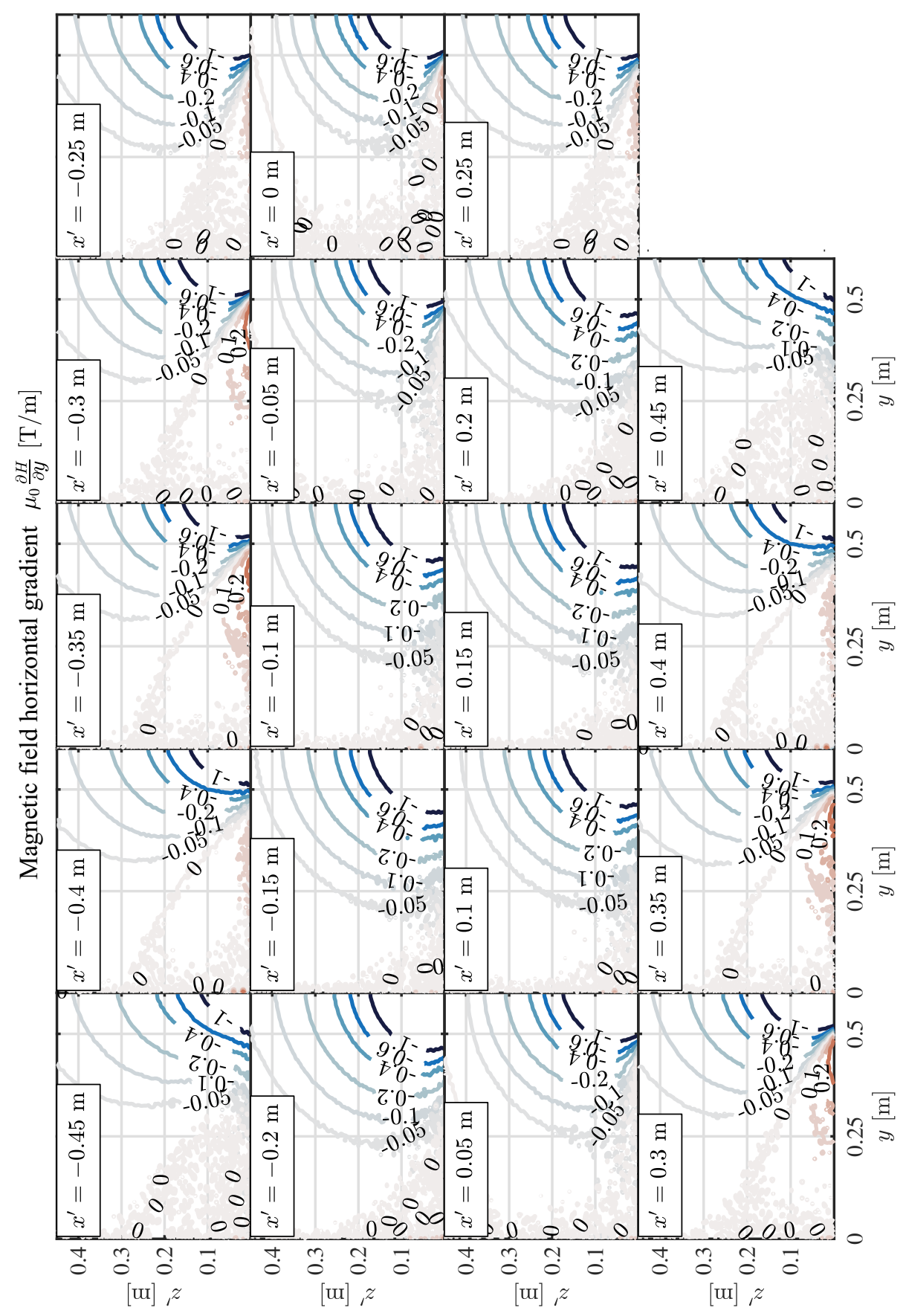




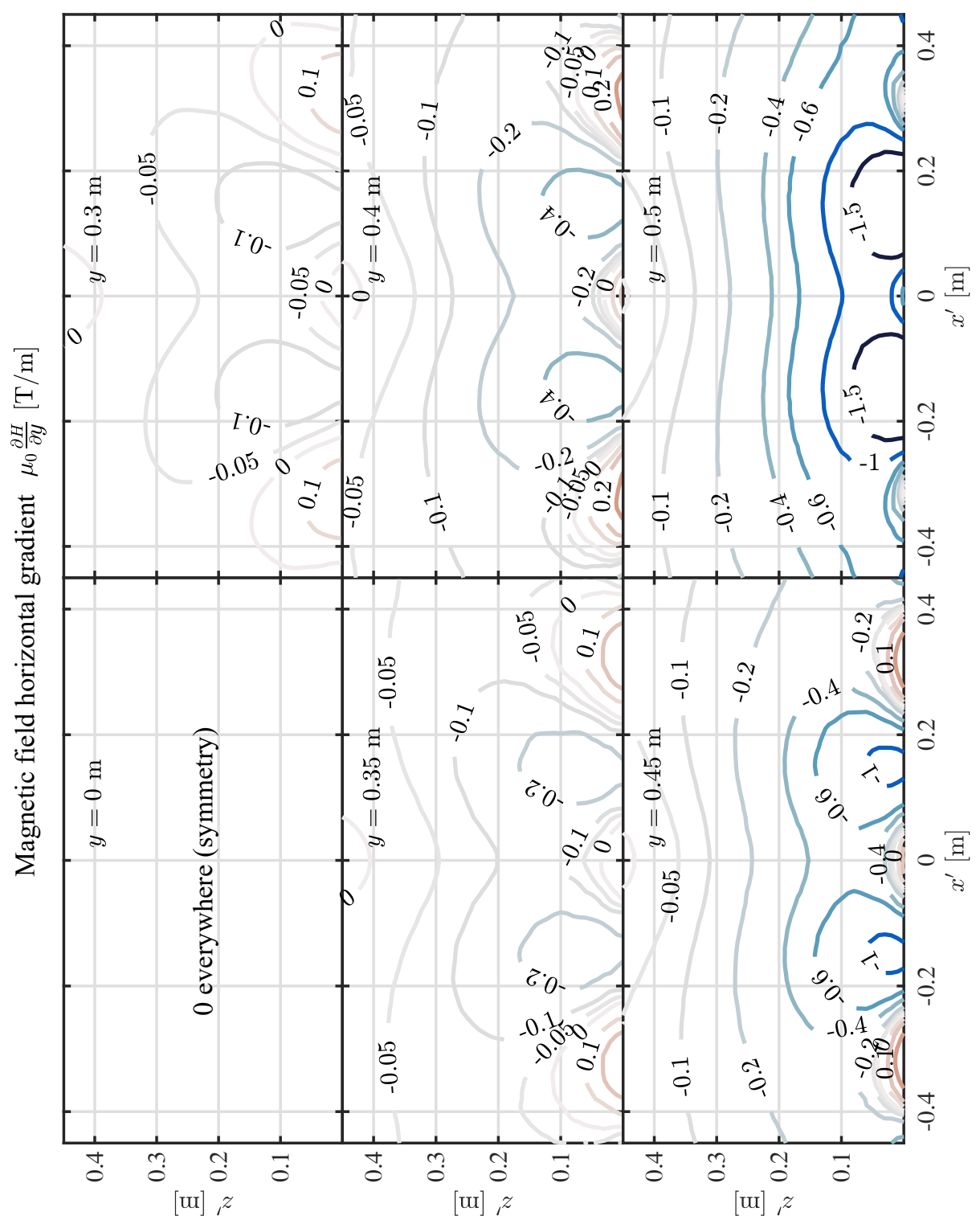




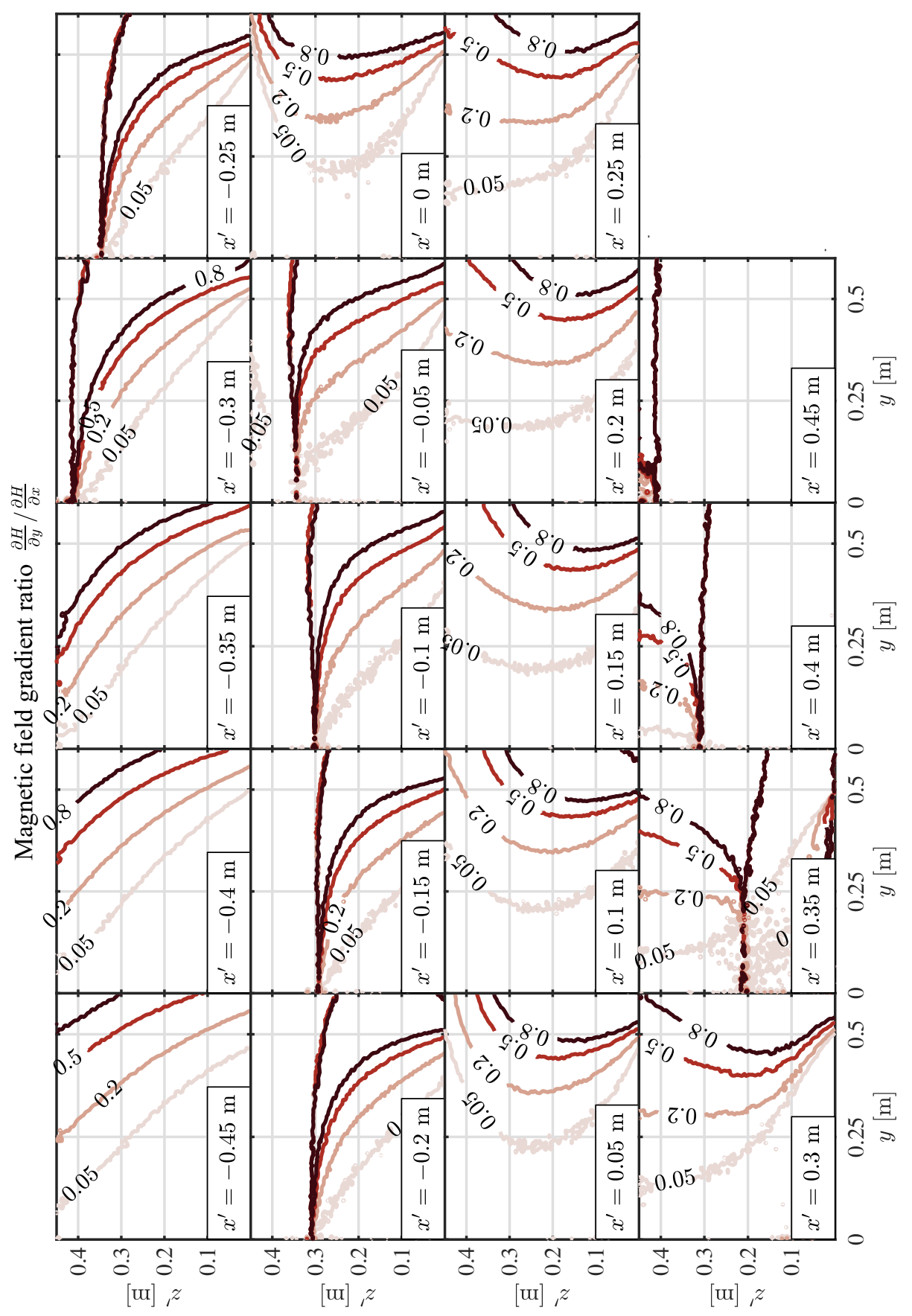




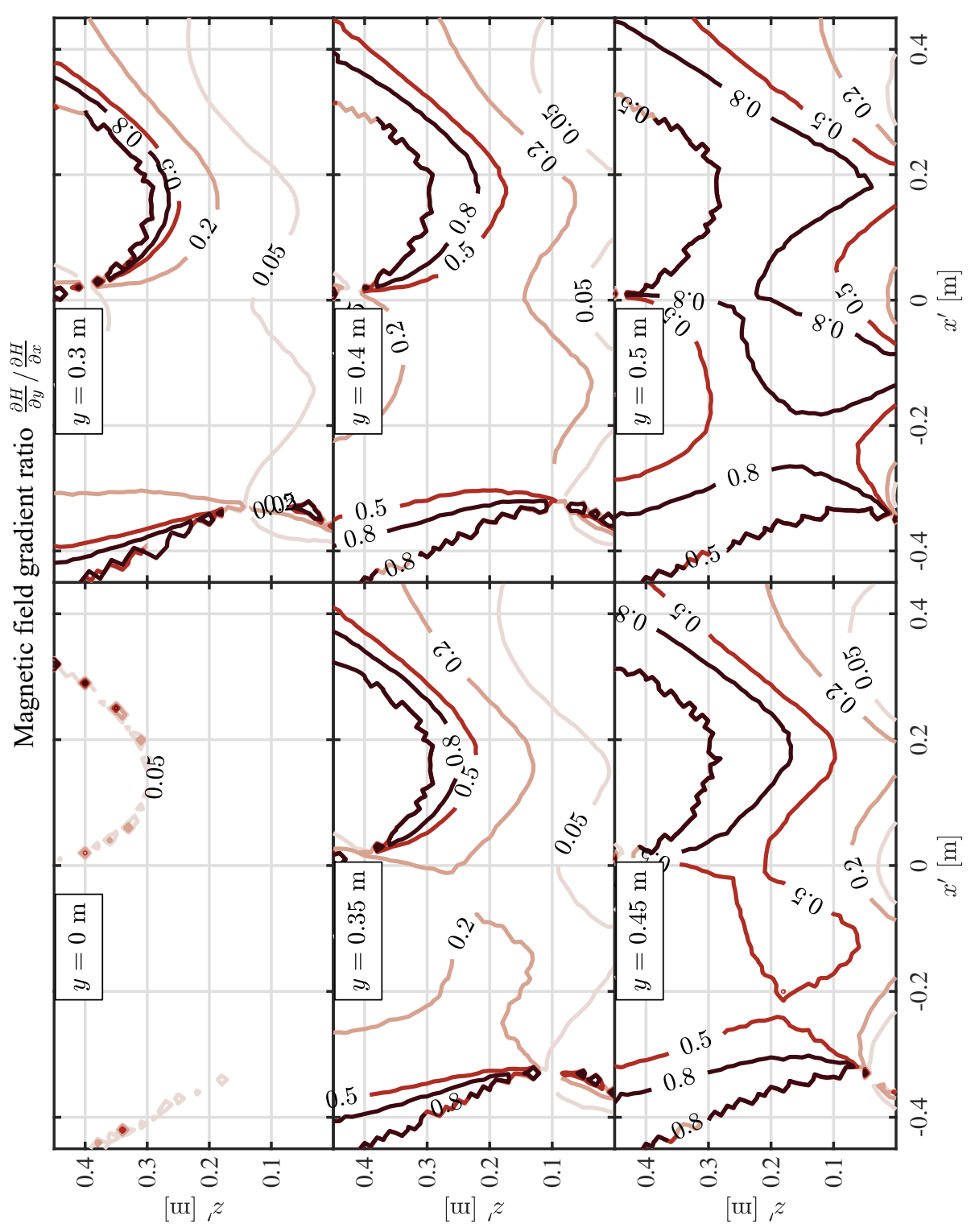




\section{Bibliography}

[1] E. Bakker, P.C. Rem and N. Fraunholcz. "Upgrading mixed polyolefin waste with magnetic density separation". In: Waste Management 29.5 (2009), pp. 1712-1717.

[2] B. Hu. "Magnetic Density Separation of polyolefin wastes". University of Delft, Delft, 2014.

[3] S. Serranti et al. "An innovative recycling process to obtain pure polyethylene and polypropylene from household waste". In: Waste Management 35 (2015), pp. 15-20.

[4] L. Muchova, E. Bakker and P.C. Rem. "Precious metals in municipal solid waste incineration bottom ash". In: Water Air Soil Pollut: Focus 9.107 (2009).

[5] E. Bakker et al. "Turning magnetic density separation into green business Using the cyclic innovation model". In: The Open Waste Management Journal 3 (2010).

[6] V. Luciani et al. "Upgrading of PVC rich wastes by magnetic density separation and hyperspectral imaging quality control". In: Waste Management 45 (2015), pp. 118-125.

[7] J.A. Oberteuffer. "Magnetic Separation: A Review of Principles, Devices, and Applications". In: IEEE Transactions on Magnetics 10 (1974), p. 223.

[8] J. Svoboda. Magnetic Techniques for the Treatment of Materials. Kluwer Academic Publishers, 2004.

[9] F.E. Luborsky and B.J. Drummond. "High Gradient Magnetic Separation: Theory Versus Experiment". In: IEEE Transactions on Magnetics 11 (1975), p. 1696.

[10] R. Gerber M. Takayasu and F.J. Friedlaender. "Magnetic separation of submicron particles". In: IEEE Transactions on Magnetics 19 (1983), p. 2112. 
[11] S.C. Trindade and H.H. Kolm. "Magnetic Desulfurization of Coal". In: IEEE Transactions on Magnetics 9 (1973), p. 310.

[12] R.R. Oder. "High gradient magnetic separation theory and applications". In: IEEE Transactions on Magnetics 12 (1976), p. 428.

[13] F.J. Friedlaender and A.S. Holman. "Development of high-gradient and open-gradient magnet separation of dry fine coal". In: IEEE Transactions on Magnetics 17 (1981), p. 3314.

[14] D.R. Kelland. "High Gradient Magnetic Separation Applied to Mineral Beneficiation”. In: IEEE Transactions on Magnetics 9 (1975), p. 307.

[15] A. Nakahira, T. Kubo and H. Murase. "Synthesis of LDH-type clay substituted with $\mathrm{Fe}$ and $\mathrm{Ni}$ ion for arsenic removal and its application to magnetic separation”. In: IEEE Transactions on Magnetics 43 (2007), p. 2442.

[16] X. Zheng, Y. Wang and D. Lu. "Effect of matrix shape on the capture of fine weakly magnetic minerals in high-gradient magnetic separation". In: IEEE Transactions on Magnetics 52.9 (2016).

[17] J.A. Oberteuffer et al. "High gradient magnetic filtration of steel mill process and waste waters". In: IEEE Transactions on Magnetics 11 (1975), p. 1591.

[18] A. Pasteur et al. "Optimization of High Gradient Magnetic Separation Filter Units for the Purification of Fermentation Products". In: IEEE Transactions on Magnetics 50 (2014), p. 6818417.

[19] D. Melville, F. Paul and S. Roath. "High Gradient Magnetic Separation of Red Cells from Whole Blood”. In: IEEE Transactions on Magnetics 11 (1975), p. 1701.

[20] A. Hultgren et al. "High-yield cell separations using magnetic nanowires". In: IEEE Transactions on Magnetics 40 (2004), p. 2988.

[21] K. Tsukada et al. "Using Magnetic Field Gradients to Shorten the AntigenAntibody Reaction Time for a Magnetic Immunoassay". In: IEEE Transactions on Magnetics 55 (2019), p. 8637765.

[22] D. Fletcher et al. "Eddy-current separation of non-ferrous conductors and non-conductors: theory and initial experiments". In: IEEE Transactions on Magnetics 27 (1991), p. 5375.

[23] J. Cui and E. Forssberg. "Mechanical recycling of waste electric and electronic equipment: A review". In: Journal of Hazardous Materials 99 (2003), p. 243.

[24] R. Kaiser and G. Miskolczy. "Some applications of ferrofluid magnetic colloids". In: IEEE Transactions on Magnetics 6.3 (1970), pp. 694-698. 
[25] Alex M. van Silfhout, H. Engelkamp and B.H. Erné. "Colloidal Stability of Aqueous Ferrofluids at 10 T". In: The Journal of Physical Chemistry Letters 11.15 (2020), pp. 5908-5912.

[26] Innovative Magnetic Density Separation for the optimal use of resources and energy. 2015.

[27] K. Halbach. "Design of permanent multipole magnets with oriented rare earth cobalt material". In: Nuclear Instruments and Methods 169 (1980), pp. 1-10.

[28] J. Mallinson. "One-sides fluxes - A magnetic curiosity?" In: IEEE Transactions on Magnetics 9.4 (1973), pp. 678-682.

[29] P.C. Rem. personal communication. 2020.

[30] J.X. Jin, S.X. Dou, H.K. Liu et al. "A high gradient magnetic separator fabricated using Bi-2223/Ag HTS tapes". In: IEEE Transactions on Applied Superconductivity 9.2 (1999).

[31] R. Gerber and M.H. Watmough. "A design of a superconducting split-coil open-gradient magnetic separator". In: IEEE Transactions on Magnetics 21.2053 (1985).

[32] J. Iannicelli et al. "Magnetic separation of kaolin clay using a high temperature superconducting magnet system". In: IEEE Transactions on Applied Superconductivity 7.1061 (1997).

[33] J. Boehm et al. "Deflection of weakly magnetic materials by superconducting OGMS”. In: IEEE Transactions on Magnetics 24.1674 (1988).

[34] D.D. Jackson. "Processing of China Clays Using a Commercial-Scale, Conduction-Cooled Superconducting Magnetic Separation System". In: IEEE Transactions on Magnetics 49.7 (2013), pp. 3438-3440.

[35] K.J. Binns. The Analytical and Numerical Solution of Electric and Magnetic Fields. Wiley, 1992.

[36] H. Gobel. "Inventarisatie scheidingstechnieken harde polyolefinen in polypropyleen (PP) en polyethyleen (PE)". In: The Public Waste Agency of Flanders (OVAM) (2009).

[37] V. Zermeño and F. Grilli. "3D modelling and simulation of 2G HTS stacks and coils”. In: Superconductor Science and Technology 27.044025 (2014), pp. 99-116.

[38] Y. Iwasa. Case Studies in Superconducting Magnets. Springer, 2009.

[39] M.N. Wilson. Superconducting magnets. Clarendon Press, 1987. 
[40] P.C. Rem and P. Berkhout. Splitter for Magnetic Density Separation. International Patent No. WO 2017/111583 A1. 2017.

[41] H.S. Shin et al. "Bending strain characteristics of critical current in REBCO CC tapes in different modes". In: Physica C: Superconductivity 469.15-20 (2009), pp. 1467-1471.

[42] J.W. Ekin. Experimental Techniques for Low Temperature Measurements. Oxford. Univ. Press, 2006.

[43] F. Paschen. "Ueber die zum Funkenübergang in Luft, Wasserstoff und Kohlensäure bei verschiedenen Drucken erforderliche Potentialdifferenz". In: Annalen der Physik 273.5 (1889), pp. 69-75.

[44] U.P. Trociewitz, D.K. Hilton E. Bosque et al. "Bi-2212 Coil Technology at NHMFL". In: Magnet Technology 25, 2017.

[45] H.H.J. ten Kate. personal communication. 2016.

[46] J. Fleiter and A. Ballarino. Parameterization of the critical surface of REBCO conductors from Fuijikura. Tech. rep. CERN. 2014.

[47] A. van Silfhout and B. Erné. "Magnetic detection of nanoparticle sedimentation in magnetized ferrofluids". In: Journal of Magnetism and Magnetic Materials 472 (2019), pp. 53-58.

[48] G. Brown et al. "Wiggler and undulator magnets - a review". In: Nucl. Instrum. Methods Phys. Res. 208.1-3 (1983), pp. 65-77.

[49] E.J. Jaeschke, E. Gluskin, N. Mezentsev et al. Synchrotron Light Sources and Free-Electron Lasers. Springer Nature Switzerland AG, 2019.

[50] Thermal Insulation Products - COOLCAT 2 NW. RUAG Space, 2019.

[51] E. Kostrov, A. Bagdinov, E. Demikhov et al. "Performance Test of a G-M Cooler in Magnetic Field”. In: Physics Procedia 267 (2015), pp. 440-444.

[52] RDK-415D 4K Cryocooler Series Capacity Map. URL: www . shicryogenics . com/wp-content/uploads/2019/08/RDK-415D_Capacity_ Map.pdf (visited on 24/02/2020).

[53] K. Pieterman, A. Ketting and J. Geerse. "Ferromagnetic traces in austenitic stainless steels at $4.2 \mathrm{~K}$ ". In: Journal de Physique Colloques 45 (1984), pp. 625-631.

[54] S.P. Timoshenko. Strength of materials pt. 1, Elementary theory and problems. Princeton, 1955.

[55] C.E. Imrak and I. Gerdemeli. "An Exact Solution for the Deflection of a Clamped Rectangular Plate under Uniform Load". In: Applied Mathematical Sciences 1.43 (2007), pp. 2129-2137. 
[56] J. Hagedorn, F. Sell-Le Blanc and J. Fleischer. Handbook of Coil Winding: Technologies for efficient electrical wound products and their automated production. Springer Vieweg, 2019.

[57] S. Foner and B.B. Schwartz. Superconductor Materials Science: Metallurgy, Fabrication, and Applications. Springer Science \& Business Media, 2012.

[58] W.H. Gray and C.T. Sun. Theoretical and experimental determination of mechanical properties of superconducting wire. Oak Ridge National Laboratory, 1976.

[59] J.W. Ekin, R.E. Schramm and A.F. Clark. Effect of Strain on Epoxy Impregnated Superconducting Composites, a chapter in Nonmetallic Materials and Composites at low Temperatures. Plenum Press - New York and London, 1978.

[60] C.W. Hicks, D.O. Brodsky, E.A. Yelland et al. "Strong Increase of $T_{c}$ of $\mathrm{Sr}_{2} \mathrm{RuO}_{4}$ Under Both Tensile and Compressive Strain”. In: Science 344 (2014), p. 283.

[61] C. Barth, N. Bagrets, K.-P. Weiss et al. "Degradation free epoxy impregnation of REBCO coils and cables". In: Superconductor Science and Technology 26 (2013), p. 5.

[62] E.S. Bobrov, J.E.C. Williams and Y. Iwasa. "Experimental and theoretical investigation of mechanical disturbances in epoxy impregnated superconducting coils. 2. Shear-stress-induced epoxy fracture as the principal source of premature quenches and training- theoretical analysis". In: Cryogenics 25 (1985), pp. 307-316.

[63] A.F. Clark, W.F. Weston, V.D. Arp et al. Characterization of a superconducting coil composite and its components. National Bureau of Standards, 1976.

[64] H.M. Ledbetter and D.T. Read. "Orthorhombic constants of an $\mathrm{NbTi} / \mathrm{Cu}$ composite superconductor”. In: Journal of Applied Physics 48.5 (1977), pp. 141-146.

[65] L. Rossi. "Superconducting Magnets for the LHC Main Lattice". In: Magnet Technology 18, 2003.

[66] Y. Lvovsky, E.W. Stautner and T. Zhang. "Novel technologies and configurations of superconducting magnets for MRI". In: Superconductor Science and Technology 26 (2013), p. 093001.

[67] H.H.J. ten Kate. "Superconducting Magnets Quench Propagation and Protection". In: CERN Accelerator School on Superconductivity for Accelerators, Erice, 2013. 
[68] A.V. Tollestrup. "Care and training of superconducting magnets". In: IEEE Trans. Magn. 17 (1981), pp. 863-872.

[69] G. Pasztor and C. Schmidt. "Dynamic stress effects in technical superconductors and the "training" problem of superconducting magnets". In: Journal of Applied Physics 49 (1978), p. 886.

[70] F. Toral. Mechanical Design of Superconducting Accelerator Magnets. Tech. rep. CERN-2014-005. 2015.

[71] L. Wang et al. "Design and Construction of a Prototype Solenoid Coil for MICE Coupling Magnets". In: IEEE Transactions on Applied Superconductivity 20.3 (2010), pp. 373-376.

[72] S. Bartlett, S. Caspi and D.R. Diederich. "An R\&D Approach to the Development of Long $\mathrm{Nb}_{3} \mathrm{Sn}$ Accelerator Magnets Using the Key and Bladder Technology". In: IEEE Transactions on Applied Superconductivity 15.2 (2005), pp. 1136-1139.

[73] J.G. Kaufman. Properties of Aluminum Alloys: Tensile, Creep and Fatigue Data at High and Low Temperatures. The Aluminum Association, 1999.

[74] W. van de Camp, M.M.J. Dhallé, W.A.J Wessel et al. "Cryogenic Fatigue and Stress-strain Behavior of a Fibre Metal Laminate". In: Physics Procedia 67 (2015), pp. 1043-1048.

[75] COMSOL Materials Library 5.4. COMSOL AB, 2019.

[76] Runde Rohre Vetronit Cryo T datasheet WHA C07TVGC612. Von Roll Deutschland GmbH, 2018.

[77] C.L. Goodzeit. "Superconducting accelerator magnets". In: USPAS, 2001.

[78] J.E. Huber, D. Nowell and D.A. Hills. "On the stress analysis of a wound coil with application to electromagnet manufacture". In: The Journal of Strain Analysis for Engineering Design 42.6 (2007), pp. 447-460.

[79] J.G. Kaufman. Relational Analysis: Guidelines for Estimating High and Low Temperature Properties of Metals. ASM International, 2011.

[80] C.M. Harris. Shock and vibration handbook. McGraw-Hill, 1988.

[81] Eurocode 8: Design of structures for earthquake resistance - Part 1 : General rules, seismic actions and rules for buildings. The European Union per Regulation 305/2011, Directive 98/34/EC Directive 2004/18/EC.

[82] Magnitude / Intensity Comparison. United States Geological Survey. URL: https: / / earthquake.usgs.gov/learn/topics/mag_vs_ int.php (visited on 18/07/2019). 
[83] D. Wald et al. "Relationships between Peak Ground Acceleration, Peak Ground Velocity, and Modified Mercalli Intensity in California". In: Earthquake Spectra 15.3 (1999), pp. 557-564.

[84] Structural Mechanics Module User's Guide Version 5.4. COMSOL AB, 2019.

[85] S. Polukoshko. "Estimation of damping capacity of rubber vibration isolators under harmonic excitation". In: Vibroengineering procedia 8 (2006), pp. 50-56.

[86] A.T.A.M. de Waele. "Basic Operation of Cryocoolers and Related Thermal Machines". In: J Low Temp Phys 164 (2011), pp. 79-236.

[87] A.L. Woodcraft. "Predicting the thermal conductivity of aluminium alloys in the cryogenic to room temperature range". In: Cryogenics 45 (2005), pp. 421-431.

[88] 1st Grade Superconductor Di-BSCCO Product Sheet. Sumitomo Electric, 2016.

[89] D. S. Matsumoto, C. L. Reynolds Jr. and A. C. Anderson. "Thermal boundary resistance at metal-epoxy interfaces". In: Phys. Rev. B 16.3303 (1977).

[90] N. Suman, T.S. Datta et al. "Practical load map of two stage $1.5 \mathrm{~W}$ at $4.2 \mathrm{~K}$ (SRDK-415D) GM cryocooler". In: Conference: National Symposium of Cryogenics-25, 2014.

[91] J. Zbasnik, M.A. Green, E. H. Hoyer et al. ““'Tests of a GM cryocooler and high Tc leads for use on the ALS Superbend magnets". In: Montreal: Cryogenic Engineering Conference, 1999.

[92] G. Tomás, M. van den Broek, S. Kamyar et al. “"Experimental characterization of $\mathrm{Al}-\mathrm{Cu}$ thermal contact resistance below $50 \mathrm{~K}$ ". manuscipt in preparation.

[93] A. Verweij. CUDI: Users Manual. Tech. rep. 2007.

[94] CryoComp Version 3.01. Eckels Engineering Inc., Florence, USA.

[95] R. Poggiani. Materials and components of possible interest for cryogenic operation of Einstein Telescope. Tech. rep. ET - Einstein gravitational wave Telescope - Design Study, Cryogenics for ET. 2009.

[96] S. Capsi, J.Y. Lee, Y.I. Kim et al. Cryogenic-Coolant He ${ }^{4}$-Superconductor Interaction. Tech. rep. NASA Contractor Report 2963. 1978.

[97] J.M. Pfotenhauer et al. "Characterizing Helium Pulsating Heat Pipes". In: IOP Conf. Series: Materials Science and Engineering 502 (2019), p. 012058. 
[98] H. Boy. personal communication. 2020.

[99] A. Bergen, R. Andersen, M. Bauer et al. "Design and in-field testing of the world's first ReBCO rotor for a 3.6 MW wind generator". In: Supercond. Sci. Technol. 32 (2019), p. 125006.

[100] Bismuth Strontium Calcium Copper Oxide Sputtering Target. 25th Mar. 2020. URL: Www . americanelements . com/bi smuth-strontiumcalcium $\div 5 \mathrm{C} \% 5 \mathrm{C}$ - copper-oxide-sputtering-target 114901-61-0.

[101] N. Bagrets et al. "Thermal properties of $2 \mathrm{G}$ coated conductor cable materials". In: Cryogenics 61 (2014), pp. 8-14.

[102] M.A. Green et al. Vacuum impregnation with epoxy of large superconducting magnet structures. Plenum Press, New York, 1979.

[103] K.-J. Best and H. Hillmann. "Magnet construction by wet winding technique with ceramic material". In: IEEE Transactions on Magnetics 17 (1981), p. 5.

[104] Loctite Stycast 2850FT. Henkel, 2015.

[105] R.A. Matula. "Electrical resistivity of copper, gold, palladium, and silver". In: Journal of Physical and Chemical Reference Data 8 (1979), p. 1147.

[106] A. Nijhuis et al. "Control of contact resistance by strand surface coating in 36-strand NbTi CICCs". In: Cryogenics 41 (2001), pp. 1-7.

[107] H. Ehmler and M. Köppen. "AC modeling and impedance spectrum tests of the superconducting magnetic field coils for the Wendelstein 7-X fusion experiment”. In: Rev. Sci. Instrum. 78 (2007), p. 104705.

[108] L. Bottura. "A Practical fit for the Critical Surface of Nb-Ti”. In: IEEE Transactions on Applied Superconductivity 10 (2000), pp. 1054-1057.

[109] A. Nijhuis. "Mechanical and Electro-Magnetic Performance of $\mathrm{Nb}_{3} \mathrm{Sn} \mathrm{Su}-$ perconductor for Fusion". University of Twente, Enschede, 2016.

[110] L.D. Cooley et al. "Conductor specification and performance validation for LARP QXF magnet cables". In: IEEE Transactions on Applied Superconductivity (2017).

[111] M. Dhallé et al. "Transport and inductive critical current densities in superconducting $\mathrm{MgB}_{2}$ ”. In: Physica C 363 (2001), pp. 155-165. 


\section{Summary}

THIS thesis concerns the design of a superconducting magnet for use in Mag1 netic Density Separation (MDS). This magnet is being constructed at the University of Twente and is meant to serve in a demonstrator set-up for the separation of shredded electronic materials.

MDS is a novel separation technology, developed in the research chair of prof. Peter Rem at the University of Delft, that can be used in for example the recycling industry. The technique is based on the combination of a fluid that is strongly attracted by magnetic fields (a ferrofluid) and a magnetic field with a strong gradient in a single direction. When shredded non-magnetic particles are inserted in the fluid bed, they will move towards different stable depths in the fluid that corresponds to their mass density. This means the MDS process can separate multiple densities in one single step, for example different plastics or electronics.

The equilibrium height of a non-magnetic particle within the ferrofluid is determined by the force balance of gravity, buoyancy and the upwards force exerted by the ferrofluid. This upwards force is created because the ferrofluid is attracted towards the magnet whereas the non-magnetic particles are not. This force scales with the gradient of the magnitude of the magnetic field and with the concentration of magnetic nanoparticles in the ferrofluid. Because the gradient changes with the distance to the magnet, the force also changes with distance to the magnet, and thus each non-magnetic particle with a different mass density will have a different equilibrium height.

State-of-the-art MDS systems use permanent magnets. Compared to these magnets, superconducting magnets can offer several benefits.

Superconductors can generate a stronger magnetic field, increasing the upwards force. This allows the separation of dense particles such as lead and copper. Another advantage that comes with the stronger magnetic field is that a lower concentration of magnetic nanoparticles in the fluid can be used while maintaining a strong upwards force. This reduces operation expenditure, because the ferrofluid is expensive and must be regularly replenished because a fraction of the fluid is lost during post-processing of separated particles. 
A second advantage in using superconducting magnets for MDS results from the fact that the separation resolution scales linearly with the pole size of the magnet. This pole size is limited for permanent magnets due to practical considerations. Electromagnets, made out of coils, do not have this limitation, and thus an enhanced separation resolution is possible, a factor 2.5 for the demonstrator.

The superconducting magnet that is the subject of this thesis consists of a set of three racetrack coils of $\mathrm{NbTi} / \mathrm{Cu}$ conductor, with a length of $1.4 \mathrm{~m}$. The coils are placed adjoining in a plane. Their geometry is optimized so that the gradient of the magnetic field magnitude only changes in a single direction, over a large volume. The relative dimensions of the coils' legs ( $100 \mathrm{~mm}$ per leg) with respect to the width of each coil of $300 \mathrm{~mm}$ are optimal to meet the requirements of the magnetic field profile.

Two basic strategies can be used to ensure that feed particles move through the fluid bed in the horizontal direction. The first used a fluid flow to drag particles along. The required number of coils for such a system can be calculated by multiplying the time feed particles need to reach their equilibrium height with the flow speed.

The second strategy makes uses of a tilt of the coils with respect to the horizontal plane. This tilt creates a horizontal component of the force that the ferrofluid exerts on the feed particles. The demonstrator makes uses of this principle. For a tilted system, three is the optimal number of coils, because of edge effects that influence the horizontal gradient.

The main MDS specific mechanical design challenge of the magnet was the minimization of the distance between the flat, roughly $1 \mathrm{~m}$ by $1 \mathrm{~m}$, surfaces formed by the top surface of the coils and the ferrofluid to $50 \mathrm{~mm}$. Initially the idea was to cool the coils using a liquid helium bath, which would have resulted in a double-walled cryostat. The two flat plates of the cryostat that separate the coils from the ferrofluid would result in a significant decrease of the magnet's performance.

Because of this it was decided to switch to a conduction-cooled system with a cryocooler, so that a single walled cryostat can be used. This is possible because the heat load is small enough to be cooled using a single cryocooler via conduction. The flat cryostat wall needs to be strong enough to withstand both the atmospheric pressure as well as the force with which the fluid is attracted towards the magnets (together $1.3 \mathrm{bar}$ ). By using a stainless steel column structure that supports the flat plate, this plate can be kept relatively thin $(20 \mathrm{~mm})$. These columns pass through the cold mass and stay at room temperature.

Each coil consist of roughly 2230 turns of $1.4 \mathrm{~mm} \mathrm{NbTi/Cu}$ conductor. The magnet generates an average magnetic field at the bottom of the fluid bed of $2.0 \mathrm{~T}$. 
The Lorentz forces are supported by two machined aluminium alloy (AL5083H321) plates. During cool-down these plates shrink-fit around the coils, due to differential thermal contraction. This results in a pre-compression on the winding packs. When the coils are energized, this compression prevents the creation of a positive transverse stress. This is important to prevent the formation and propagation of cracks in the epoxy matrix. Crack formation could cause the magnet to leave the superconducting state due to heating related to energy release during crack propagation.

The required thickness of the aluminium alloy plates depends on the required pre-compression on the winding packs, and less on the longitudinal stress in the conductor or the stress in the plates themselves.

The fibreglass structure that keeps the cold mass in place is optimized to offer the required mechanical strength with as low as possible thermal conduction. G11 fibreglass is the optimum material choice when comparing materials on buckling resistance as well as on tensile strength, relative to their thermal conduction.

The coils are designed to operate at a temperature of $4.5 \mathrm{~K}$ or below. The heat balance of the system shows that this is feasible with the current design, using a single two-stage cryocooler. The coils require around two weeks to cool down from room temperature.

The three NbTi-based coils are wound at the University of Twente, using a wet-winding technique. During winding of the second coil an electrical short was created. Analysis on the effects of this short indicate that the system will need at least a day to ramp the current from zero to the operational current of $300 \mathrm{~A}$, in order to keep heating due to the short within acceptable bounds.

The system generates a gradient of $20 \mathrm{~T} / \mathrm{m}$ at the bottom of the fluid bed. This is near to the limit of what NbTi can offer at this pole size. A large enhancement of the performance is possible using for example a $\mathrm{Nb}_{3} \mathrm{Sn}$ - or ReBCO-based system. For these superconductors the potential performance depend strongly on the mechanical design of the cold mass. As mentioned, the demonstrator uses two machined aluminium alloy plates to handle the Lorentz force. These plates have the same thickness and as such the cold mass looks symmetrical. This design can be extrapolated for more powerful magnets. When the coils generate stronger forces, the plates will need to be thicker and thus the distance between the coils and fluid increases. This limits the gradient using this approach to around $36 \mathrm{~T} / \mathrm{m}$.

If on the other hand a design can be found in which the thickness of the top plate need not increase when the force increases, a gradient of up to $77 \mathrm{~T} / \mathrm{m}$ at the fluid bed can be generated. Thus further investigation into such an asymmetric approach is strongly recommended. 


\section{Samenvatting}

IT proefschrift beschrijft het ontwerp van een supergeleidende magneet voor toepassing in Magnetische Dichtheidsscheiding (MDS). De magneet wordt geconstrueerd aan de Universiteit Twente en is bedoeld voor gebruik in een demonstratorsysteem voor de scheiding van elektronisch afval.

MDS is een nieuwe scheidingstechniek, ontwikkeld in de onderzoeksgroep van prof. Peter Rem aan de Universiteit Delft, die bijvoorbeeld in de recyclingsector gebruikt wordt. De techniek is gebaseerd op de combinatie van een vloeistof die sterk aangetrokken wordt door magneetvelden (een ferrovloeistof) en een magneetveld met een sterke gradiënt in één richting. Wanneer (versnipperde) niet-magnetische deeltjes in het vloeistofbed gestopt worden gaan deze op verschillende dieptes in de vloeistof drijven, afhankelijk van de massadichtheid van de individuele deeltjes. Men kan dus een mengsel van meerdere dichtheden met het MDS proces scheiden in één enkele stap, bijvoorbeeld verschillende plastics of zoals genoemd onderdelen van elektronica.

De evenwichtshoogte in de ferrovloeistof van een niet-magnetisch deeltje met een bepaalde massadichtheid wordt bepaald door de krachtenbalans van zwaartekracht, drijfvermogen en de door de ferrovloeistof uitgeoefende opwaartse kracht. Deze opwaartse kracht ontstaat doordat de ferrovloeistof richting de magneet getrokken wordt en de niet-magnetische deeltjes niet. Deze kracht schaalt met de gradiënt van de sterkte van het magneetveld en met de concentratie van magnetische nanodeeltjes in de vloeistof. Omdat de gradiënt verandert met de afstand tot de magneet, verandert ook de kracht met afstand tot de magneet, en daardoor heeft elk niet-magnetisch deeltje met een andere massadichtheid een eigen evenwichtshoogte.

State-of-the-art MDS-systemen maken gebruik van permanente magneten. Ten opzichte van deze magneten kunnen supergeleidende magneten diverse voordelen bieden.

Zo kan ten eerste een sterker magneetveld gegenereerd worden met supergeleiders, waardoor de opwaartse magnetische kracht groter wordt. Hierdoor kunnen ook zwaardere deeltjes zoals lood en koper gescheiden worden. Een ander 
voordeel van het sterkere magneetveld is dat een lagere concentratie magnetische nanodeeltjes in de vloeistof gebruikt kan worden terwijl toch een sterke opwaartse kracht behouden blijft. Dit scheelt in de operationele kosten van het proces, want de ferrovloeistof is duur en moet regelmatig bijgevuld worden doordat een gedeelte van de vloeistof tijdens nabewerking van gescheiden deeltjes verloren gaat.

Een tweede voordeel in het gebruik van supergeleidende magneten voor MDS ligt in het feit dat de scheidingsresolutie lineair schaalt met de grootte van de polen van de magneet. Deze poolgrootte is voor permanente magneten vanwege praktische redenen gelimiteerd. Elektromagneten gemaakt van spoelen hebben deze beperking niet, en dus kan een hogere scheidingresolutie gehaald worden, een factor 2.5 voor de demonstrator.

De supergeleidende magneet waarvan het ontwerp in dit proefschrift beschreven is omvat een set van drie racetrack spoelen van $\mathrm{NbTi} / \mathrm{Cu}$-geleider, met een lengte van $1.4 \mathrm{~m}$. De spoelen zijn naast elkaar geplaatst in een vlak. De geometrie van de spoelen is zo geoptimaliseerd zodat het magneetveld een gradiënt van haar sterkte heeft die slechts in één richting significant verandert, over een groot volume. De relatieve afmetingen van de benen van de spoelen ( $100 \mathrm{~mm}$ per been) ten opzichte van de breedte van elke spoel van $300 \mathrm{~mm}$ zijn optimaal om aan de eisen van het magneetveldprofiel te voldoen.

Er zijn twee strategieën die gebruikt kunnen worden om te zorgen dat afvaldeeltjes in horizontale richting door het vloeistofbed bewegen. De eerste gebruikt een stromende vloeistof om deeltjes mee te trekken. Voor zo'n systeem is het aantal benodigde spoelen te berekenen door de tijd die deeltjes nodig hebben om hun evenwichtshoogtes te bereiken te vermenigvuldigen met de stroomsnelheid van de vloeistof.

De tweede strategie zet de spoelen onder een hoek ten opzichte van het horizontale vlak. Door deze tilt ontstaat er een horizontale component van de kracht die de ferrovloeistof uitoefent op de deeltjes. De demonstrator maakt gebruik van dit principe. Het blijkt dat voor een gekanteld systeem het optimale aantal spoelen drie is, vanwege randeffecten die de horizontale gradiënt beïnvloeden.

De grootste specifieke uitdaging in het mechanische ontwerp lag in het minimaliseren van de afstand tussen het bovenvlak van de spoelen en de ferrovloeistof tot $50 \mathrm{~mm}$. Oorspronkelijk was het de bedoeling om de spoelen te koelen in een bad van vloeibare helium. Dit zou een dubbelwandige cryostaat betekenen. De twee vlakke platen $(\sim 1 \mathrm{~m}$ bij $1 \mathrm{~m})$ van de cryostaat die de spoelen en de ferrovloeistof dan scheiden zouden een significante vermindering van de prestaties tot gevolg hebben.

Daarom is besloten om tot een geleidings-gekoeld systeem met een cryocooler over te gaan, waardoor er slecht een enkele wand nodig is. Dit kan omdat de 
warmtebelasting klein genoeg is om weg te koelen met een enkele cryocooler via geleiding. De vlakke cryostaatwand moet sterk genoeg zijn om zowel de atmosferische druk als wel de kracht waarmee de vloeistof naar de magneet getrokken wordt te weerstaan (samen 1.3 bar). Door het gebruik van roestvrijstalen kolommen die de vlakke plaat ondersteunen kan de plaat relatief dun gehouden worden $(20 \mathrm{~mm})$. Deze kolommen passeren door gaten in de koude massa en blijven zelf op kamertemperatuur.

Elke spoel bestaat uit ongeveer 2230 wikkelingen $\mathrm{NbTi} / \mathrm{Cu}$ geleider van 1.4 $\mathrm{mm}$. De magneet wekt een gemiddeld magneetveld op de bodem van de ferrovloeistof op van 2.0 T. De Lorentzkrachten worden opgevangen door twee bewerkte platen van een sterke aluminiumlegering (AL5083-H321). Tijdens het afkoelen van het systeem krimpen deze platen vast om de spoelen, vanwege verschillen in thermische krimp, en zorgen zo voor een compressie. Wanneer de spoelen bekrachtigd worden voorkomt deze compressie een positieve transversale spanning in het wikkelpakket. Dit is belangrijk om de vorming en progressie van scheuren in de epoxy matrix van de spoelen te voorkomen. Scheurvorming zouden namelijk de supergeleider kunnen verhitten door depositie van energie en daarmee zou het systeem de supergeleidende toestand ongewenst kunnen verlaten.

De benodigde dikte van de aluminiumlegering platen hangt af van de benodigde pre-compressie van de spoelen, en minder van de trekspanning in de geleider of in de platen zelf.

De glasvezel structuur die de koude massa op zijn plek binnen de cryostaat houdt is geoptimaliseerd om de benodigde mechanische sterkte te bieden met een zo laag mogelijke warmtebelasting op de koude massa. G11 glasvezel blijkt zowel de beste keus te zijn wanneer materialen op treksterkte als wel knikbestendigheid ten opzicht van de warmtegeleidende eigenschappen vergeleken worden.

De spoelen zijn ontworpen om te werken op een temperatuur van niet meer dan 4.5 K. Berekeningen naar de warmtebalans van het systeem laten zien dat dat haalbaar moet zijn met het gekozen ontwerp. De spoelen hebben ongeveer twee weken nodig om af te koelen vanaf kamertemperatuur.

De drie NbTi spoelen zijn vervaardigd aan de Universiteit Twente met een 'wet-winding' techniek. Tijdens het wikkelproces van de tweede spoel is er een kortsluiting ontstaan. Analyse van de effecten als gevolg van deze sluiting wijst uit dat het systeem waarschijnlijk minimaal een dag nodig heeft om de stroom van nul naar de operationele stroom van $300 \mathrm{~A}$ te brengen, om opwarming als gevolg van de kortsluiting binnen acceptabele waardes te houden.

Het systeem genereert een gradiënt van $20 \mathrm{~T} / \mathrm{m}$ op de bodem van de vloeistoftank. Meer is er niet te verwachten van een NbTi-gebaseerde magneet met deze poolgrootte. Een sterkte toename van de prestaties is mogelijk met bijvoor- 
beeld $\mathrm{Nb}_{3} \mathrm{Sn}$ - of ReBCO-gebaseerde systemen. Voor deze supergeleiders hangen de potentiële prestaties erg af van het mechanische ontwerp van de koude massa.

Zoals vermeld maakt de demonstrator gebruik van aluminiumlegering platen om de Lorentz kracht op te vangen. Deze hebben dezelfde dikte, en vormen zo een symmetrisch geheel. Dit ontwerp kan geëxtrapoleerd worden naar krachtigere magneten. Wanneer de spoelen grotere krachten opwekken moeten deze platen dan dikker zijn en neemt de afstand tussen spoelen en vloeistof toe. Dit limiteert de gradiënt voor deze aanpak tot ongeveer $36 \mathrm{~T} / \mathrm{m}$.

Als er echter een ontwerp gevonden kan worden waarin de dikte van de bovenste plaat niet hoeft toe te nemen wanneer de kracht hoger wordt, dan kan een gradiënt van wel zo'n $77 \mathrm{~T} / \mathrm{m}$ opgewekt worden. Het wordt dus zeer aangeraden om zulks een asymmetrische aanpak nader te onderzoeken. 


\section{Acknowledgements}

I would like to spend a few words to express my thanks to those that supported me in the past years, be it directly or indirectly, to help make this research possible.

Marc, thanks for your guidance and friendship. I enjoyed discussing topics related to physics or totally different subjects. Let's meet again in the future over a beer or two!

Marcel and Herman, thank you for giving me the opportunity to do my $\mathrm{PhD}$ in this interesting topic, with what I felt was a large amount of freedom. You managed to convince me of the attractive work that lies in the fields of superconductors and cryogenics, and of its importance.

Sander, I really enjoyed working with you and learning part of your heap of knowledge. Always friendly and patient, always a pleasure to work with.

Gonçalo, Anne, Jeroen, Sander, Ruben, Cris, Peng, Hidde, Jianfeng, Tiemo, Anvar, Sahil, Nando, Erik, Harry, Ans, Arend, Srini, Koen, Tommaso, Nir, Konstantin, Chao, Roger, Haishan, Izak, Bas, Thomas, Teun, Simon, Peter, Rick, Abhishek, Keyang, Wei, Steven, Jorick and Anna: thanks for making my time at UTwente fun and interesting both during and outside of working hours. I look forward to meeting again in the future.

Berry, Olivier, Jonathan, Marion, Jip, Maureen, Jelle, Daan, Rick, Judith, Friso, Alexander, Vincent, Geeske, Herman, Levon, Mathijs, Lianne, Selmar, Pjotr, Michiel, Marjolein, Elroy, Thomas, Paulina, Sabina, Esther, Carmen: I am happy to have you as my friends, thank you for your support.

My family, Mam, Pap, Leanne, Tom, Annelin and Elisa, thank you for always being there for me. 